\title{
Algebraic combinatorics
}

\author{
RWTH Aachen, WS 2021 \\ RWTH Aachen, SS 2017, WS 2017 \\ Leibniz-Universität Hannover, SS 2013 \\ Universität Duisburg-Essen, SS 2011 \\ Jürgen Müller
}




\section{Contents}

0 What is combinatorics? .................. 1

I Counting $\quad 6$

1 Sets . . . . . . . . . . . . . . . . . . 6

2 Selections ...................... . . . . 10

3 Partitions and permutations . . . . . . . . . . . . . 16

4 Difference calculus . . . . . . . . . . . . . . . . . . 22

\section{Posets 26}

5 Partially ordered sets . . . . . . . . . . . . . . . 26

6 Modular lattices . . . . . . . . . . . . . . . . . . . . . . . . 34

7 Benson-Conway Theorem . . . . . . . . . . . . . . . . . 38

$\begin{array}{lll}\text { III Incidence algebras } & 47\end{array}$

8 Incidence algebras f . . . . . . . . . . . . . . . . . 47

9 Möbius functions . . . . . . . . . . . . . . . . . . 54

10 Inclusion-exclusion . . . . . . . . . . . . . . . . . 58

11 Finite incidence algebras . . . . . . . . . . . . . . . . . 62

\section{$\begin{array}{lll}\text { IV Generating functions } & 69\end{array}$}

12 Power series . . . . . . . . . . . . . . . . . . . . . . . . . . 69

13 Generating functions . . . . . . . . . . . . . . 73

14 Partition identities . . . . . . . . . . . . . . . 77

$\begin{array}{lll}\text { V Group actions } & 81\end{array}$

15 Actions . . . . . . . . . . . . . . . . . . 81

16 Action on maps . . . . . . . . . . . . . . . . . . . . 89

17 Polya's Theorem . . . . . . . . . . . . . . . . . . . . . . 94

18 Counting patterns $\ldots \ldots \ldots \ldots \ldots$

$\begin{array}{lll}\text { VI Exercises and references } & 108\end{array}$

19 Exercises for Part I (in German) . . . . . . . . . . . . . . . . 108

20 Exercises for Part II (in German) . . . . . . . . . . . . . . . . 115 
21 Exercises for Part III (in German) . . . . . . . . . . . . . . . 116

22 Exercises for Part IV (in German) . . . . . . . . . . . . . . . . . 118

23 Exercises for Part V (in German) . . . . . . . . . . . . . . . . . 124

24 References . . . . . . . . . . . . . . . . . . . . . . . 128 


\section{What is combinatorics?}

- 'Combinatorial theory' is the name now given to the subject formerly called 'combinatorial analysis' or 'combinatorics', though these terms are still used by many people. Like many branches of mathematics, its boundaries are not clearly defined, but the central problem may be considered that of arranging objects according to specified rules and finding out in how many ways this may be done.

If the specified rules are very simple, then the chief emphasis is on the enumeration of the number of ways in which the arrangement may be made. If the rules are subtle or complicated, the chief problem is whether or not such arrangements exist, and to find methods for constructing the arrangements. An intermediate area is the relationship between related choices, and a typical theorem will assert that the maximum for one kind of choice is equal to the minimum for another kind.

$[6, p . i x]$

- The basic problem of enumerative combinatorics is that of counting the number of elements of a finite set $S$. This definition, as it stands, tells us little about the subject since virtually any mathematical problem can be cast in these terms. In a genuine enumerative problem, the elements of $S$ will usually have a rather simple combinatorial definition and very little additional structure. It will be clear that $S$ has many elements, and to main issue will be to count or estimate them all. ...

There has been an explosive growth in combinatorics in recent years. ... One important reason for this growth has been the fundamental role that combinatorics plays as a tool in computer science and related areas. A further reason has been the prodigious effort ... to bring coherence and unity to the discipline of combinatorics. ... Enumerative combinatorics has been greatly elucidated by this effort, as has its role in such areas of mathematics as finite group theory, representation theory, commutative algebra, algebraic geometry, and algebraic topology.

$[11, p .1]$

(0.1) Example: Fibonacci numbers. a) The following problem was posed in the medieval book 'Liber abbaci' [Leonardo da Pisa 'Fibonacci', 1202]: Any female rabbit gives birth to a couple of rabbits monthly, from its second month of life on. If there is a single couple in the first month, how many are there in month $n \in \mathbb{N}$ ?

Hence let $\left[F_{n} \in \mathbb{N}_{0} ; n \in \mathbb{N}_{0}\right]$ be the linear recurrent sequence of degree 2 given by $F_{0}:=0$ and $F_{1}:=1$, and $F_{n+2}:=F_{n}+F_{n+1}$ for $n \in \mathbb{N}_{0}$. Thus we obtain the sequence of Fibonacci numbers, see also Table 1:

\begin{tabular}{|l||r|r|r|r|r|r|r|r|r|r|r|r|r|r|r|r|}
\hline$n$ & 0 & 1 & 2 & 3 & 4 & 5 & 6 & 7 & 8 & 9 & 10 & 11 & 12 & 13 & 14 & 15 \\
\hline$F_{n}$ & 0 & 1 & 1 & 2 & 3 & 5 & 8 & 13 & 21 & 34 & 55 & 89 & 144 & 233 & 377 & 610 \\
\hline
\end{tabular}

To find a closed formula for $F_{n}$, and to determine the growth behavior of $F_{n}$, we proceed as follows; see also (13.1) for a different treatment: 
Table 1: Fibonacci numbers.

\begin{tabular}{|l|l|}
\hline$n$ & $F_{n}$ \\
\hline \hline 1 & 1 \\
2 & 1 \\
4 & 3 \\
8 & 21 \\
16 & 987 \\
32 & 2178309 \\
64 & 10610209857723 \\
128 & 251728825683549488150424261 \\
256 & 141693817714056513234709965875411919657707794958199867 \\
\hline
\end{tabular}

Letting $A:=\left[\begin{array}{ll}\cdot & 1 \\ 1 & 1\end{array}\right] \in \mathbb{R}^{2 \times 2}$ we have $A \cdot\left[F_{n}, F_{n+1}\right]^{\mathrm{tr}}=\left[F_{n+1}, F_{n+2}\right]^{\mathrm{tr}}$, thus $\left[F_{n}, F_{n+1}\right]^{\text {tr }}=A^{n} \cdot\left[F_{0}, F_{1}\right]^{\text {tr }}$ for $n \in \mathbb{N}_{0}$. We have $\chi_{A}=\operatorname{det}\left(X E_{2}-A\right)=X^{2}-$ $X-1=\left(X-\rho_{+}\right)\left(X-\rho_{-}\right) \in \mathbb{R}[X]$, where $\rho_{ \pm}:=\frac{1}{2}(1 \pm \sqrt{5}) \in \mathbb{R}$. From $\operatorname{ker}(A-$ $\left.\rho_{ \pm} E_{2}\right)=\left\langle\left[1, \rho_{ \pm}\right]^{\operatorname{tr}}\right\rangle_{\mathbb{R}}$ we get the diagonalising matrix $P:=\left[\begin{array}{cc}1 & 1 \\ \rho_{+} & \rho_{-}\end{array}\right] \in \mathrm{GL}_{2}(\mathbb{R})$, where $P^{-1}:=\frac{1}{\rho_{-}-\rho_{+}} \cdot\left[\begin{array}{cc}\rho_{-} & -1 \\ -\rho_{+} & 1\end{array}\right]$. Thus we have $P^{-1} A^{n} P=\left(P^{-1} A P\right)^{n}=$ $\left(\operatorname{diag}\left[\rho_{+}, \rho_{-}\right]\right)^{n}=\operatorname{diag}\left[\rho_{+}^{n}, \rho_{-}^{n}\right]$, implying

$$
A^{n}=P \cdot \operatorname{diag}\left[\rho_{+}^{n}, \rho_{-}^{n}\right] \cdot P^{-1}=\frac{1}{\rho_{-}-\rho_{+}} \cdot\left[\begin{array}{cc}
\rho_{+}^{n} \rho_{-}-\rho_{+} \rho_{-}^{n} & \rho_{-}^{n}-\rho_{+}^{n} \\
\rho_{+}^{n+1} \rho_{-}-\rho_{+} \rho_{-}^{n+1} & \rho_{-}^{n+1}-\rho_{+}^{n+1}
\end{array}\right] .
$$

Hence we have the Moivre-Binet Formula [1718, 1843] ([Bernoulli, 1728]) $F_{n}=\frac{\rho_{-}^{n}-\rho_{+}^{n}}{\rho_{-}-\rho_{+}}=\frac{1}{\sqrt{5}}\left(\rho_{+}^{n}-\rho_{-}^{n}\right)$. Since $\left|\rho_{+}\right|>1$ and $\left|\rho_{-}\right|<1$ this yields $\lim _{n \rightarrow \infty} \frac{F_{n} \cdot \sqrt{5}}{\rho_{+}^{n}}=1$, in particular the $F_{n}$ grow exponentially.

b) The number $\rho_{+}:=\frac{1}{2}(1+\sqrt{5}) \in \mathbb{R}$ is called the golden ratio, featuring in the following classical problem: How has a line segment to be cut into two pieces, such that length ratio between the full segment and the longer piece coincides with the length ratio between the longer and the shorter piece? Assume that the line segment has length 1 , and letting $\frac{1}{2}<x<1$ be the length of the longer piece, we thus have $\frac{1}{x}=\frac{x}{1-x}$, or equivalently $x^{2}+x-1=0$, which yields $x=\frac{1}{2}(-1+\sqrt{5}) \in \mathbb{R}$ as the unique positive solution. Thus the above ratio indeed equals $\frac{x}{1-x}=\frac{1}{x}=\frac{2}{-1+\sqrt{5}}=\frac{1}{2}(1+\sqrt{5})=\rho_{+} \sim 1.61803$.

From the above we infer $\lim _{n \rightarrow \infty} \frac{F_{n+1}}{F_{n}}=\rho_{+}$, saying that the Fibonacci numbers yield (good) rational approximations of the golden ratio: For example, we have 
Table 2: Paths in a square grid.

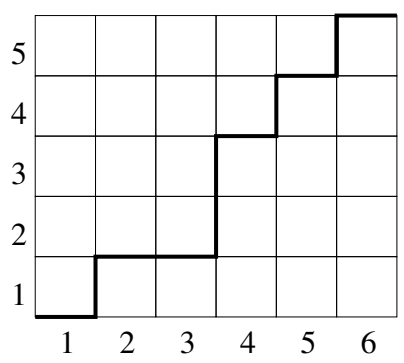

\begin{tabular}{|c|c|c|c|c|}
\hline & 6 & 21 & 5 & \\
\hline 1 & 5 & 15 & 35 & \\
\hline 1 & 4 & 10 & 20 & \\
\hline 1 & 3 & 6 & 10 & \\
\hline 2 & 2 & 3 & 4 & 5 \\
\hline & 1 & 1 & 1 & \\
\hline
\end{tabular}

$\frac{F_{8}}{F_{7}}=\frac{21}{13} \sim 1.61538, \frac{F_{9}}{F_{8}}=\frac{34}{21} \sim 1.61905, \frac{F_{10}}{F_{9}}=\frac{55}{34} \sim 1.61765, \frac{F_{11}}{F_{10}}=\frac{89}{55} \sim$ $1.61818, \frac{F_{12}}{F_{11}}=\frac{144}{89} \sim 1.61798, \frac{F_{13}}{F_{12}}=\frac{233}{144} \sim 1.61806, \frac{F_{14}}{F_{13}}=\frac{377}{233} \sim 1.61803$.

(0.2) Example: Shortest paths. Given an $(m \times n)$-square grid, where $m, n \in$ $\mathbb{N}_{0}$, we want to determine the number of shortest paths from the bottom leftmost vertex to the upper rightmost vertex, see Table 2 for the case $m=6$ and $n=5$. Hence any of these paths is of length $m+n$, consisting of a series of $m$ right moves and $n$ up moves, which is uniquely determined by the $m$-subset indicating the right moves, or alternatively by the $n$-subset indicating the up moves.

Using coordinates, we count the number $f(i, j) \in \mathbb{N}$, where $i \in\{0, \ldots, m\}$ and $j \in\{0, \ldots, n\}$, of ways of getting from the bottom leftmost vertex $[0,0]$ to vertex $[i, j]$. Hence we have $f(0, j)=f(i, 0)=1$, and $f(i, j)=f(i-1, j)+f(i, j-1)$ for $i, j \geq 1$, yielding the pattern as shown in Table 2 for $m=n=5$. We now derive a concise description of $f(m, n)$; see also (2.6):

The paths in question have length $m+n$, and are uniquely described by the $m$-set of positions where the right moves are made, or equivalently are uniquely described by the (complementary) $n$-set of positions where the up moves are made. Hence we infer that $f(m, n)=\left(\begin{array}{c}m+n \\ m\end{array}\right)=\left(\begin{array}{c}m+n \\ n\end{array}\right)$ is a binomial coefficient, see (2.1). The above recursion translates into $\left(\begin{array}{c}m+n \\ m\end{array}\right)=\left(\begin{array}{c}m+n-1 \\ m-1\end{array}\right)+\left(\begin{array}{c}m+n-1 \\ n-1\end{array}\right)=$ $\left(\begin{array}{c}m+n-1 \\ m-1\end{array}\right)+\left(\begin{array}{c}m+n-1 \\ m\end{array}\right)$, for $m, n \geq 1$.

(0.3) Example: Parity of binomial coefficients. From the above considerations we derive palindromicity $\left(\begin{array}{l}n \\ k\end{array}\right)=\left(\begin{array}{c}n \\ n-k\end{array}\right)$, for $n \in \mathbb{N}_{0}$ and $k \in\{0, \ldots, n\}$, and the triangle identity $\left(\begin{array}{l}n \\ k\end{array}\right)=\left(\begin{array}{c}n-1 \\ k-1\end{array}\right)+\left(\begin{array}{c}n-1 \\ k\end{array}\right)$, for $n \in \mathbb{N}$ and $k \in\{1, \ldots, n\}$. This shows by way of example how the 'path model' can be used to prove identities for binomial coefficients.

The triangle identity gives rise to the Pascal triangle shown in Table 3, allowing to compute binomial coefficients by using additions alone, but no multiplications. The Pascal triangle has a rich structure, and actually binomial 
Table 3: The Pascal triangle.

\begin{tabular}{|r||rrrrrrrrrrr|}
\hline$n \backslash k$ & 0 & 1 & 2 & 3 & 4 & 5 & 6 & 7 & 8 & 9 & 10 \\
\hline \hline 0 & 1 & & & & & & & & & & \\
1 & 1 & 1 & & & & & & & & & \\
2 & 1 & 2 & 1 & & & & & & & & \\
3 & 1 & 3 & 3 & 1 & & & & & & & \\
4 & 1 & 4 & 6 & 4 & 1 & & & & & & \\
5 & 1 & 5 & 10 & 10 & 5 & 1 & & & & & \\
6 & 1 & 6 & 15 & 20 & 15 & 6 & 1 & & & & \\
7 & 1 & 7 & 21 & 35 & 35 & 21 & 7 & 1 & & & \\
8 & 1 & 8 & 28 & 56 & 70 & 56 & 28 & 8 & 1 & & \\
9 & 1 & 9 & 36 & 84 & 126 & 126 & 84 & 36 & 9 & 1 & \\
10 & 1 & 10 & 45 & 120 & 210 & 252 & 210 & 120 & 45 & 10 & 1 \\
\hline
\end{tabular}

coefficients fulfill lots of (miraculous) identities. For example, we may ask for the parity of $\left(\begin{array}{l}n \\ k\end{array}\right)$, and in particular how many of the entries in row $n$ of the Pascal triangle are odd; see also (2.7) for a more general treatment:

i) The positions of the odd entries in the Pascal triangle yield the following repeating pattern, see Table 4 : If the $2^{i}$ rows $0, \ldots, 2^{i}-1$ are given, where $i \in \mathbb{N}_{0}$, then the next rows $2^{i}, \ldots, 2^{i+1}-1$ are obtained by copying down the given rows by $2^{i}$ rows, and then copying the new part to the right by $2^{i}$ columns:

By the binomial formula we have $(X+1)^{n}=\sum_{k=0}^{n}\left(\begin{array}{l}n \\ k\end{array}\right) X^{k} \in \mathbb{Z}[X]$, so that row $n$ of the Pascal triangle can be interpreted as the polynomial $(X+1)^{n} \in \mathbb{Z}[X]$. Hence by reducing modulo 2 , the parity pattern of the binomial coefficients in row $n$ can be interpreted as the polynomial $(X+1)^{n} \in \mathbb{F}_{2}[X]$. Recall that we have $(X+1)^{2}=X^{2}+1 \in \mathbb{F}_{2}[X]$.

Now let $n=\sum_{i=0}^{l} a_{i} \cdot 2^{i}$ be the 2 -adic representation of $n$, where $l=l(n)=$ $\left\lfloor\log _{2}(n)\right\rfloor \in \mathbb{N}_{0}$ and $a_{i}=a_{i}(n) \in\{0,1\}$; thus $a_{l}=1$. Letting $n^{\prime}:=n-2^{l}$ we have $n^{\prime} \in\left\{0, \ldots, 2^{l}-1\right\}$, and we get $(X+1)^{n}=(X+1)^{2^{l}+n^{\prime}}=(X+1)^{2^{l}}(X+1)^{n^{\prime}}=$ $\left(X^{2^{l}}+1\right)(X+1)^{n^{\prime}}$. Hence the positions of the odd entries in row $n$ are found as those in row $n^{\prime}$, occupying positions $\left\{0, \ldots, 2^{l}-1\right\}$ (this is the piece copied down), as well as those in row $n^{\prime}$ shifted by $2^{l}$ steps to the right, occupying positions $\left\{2^{l}, \ldots, 2^{l+1}-1\right\}$ (this is the piece copied to the right).

In particular, we observe that the Pascal triangle with $2^{i}$ rows, where $i \in \mathbb{N}_{0}$, has a total of $3^{i}$ odd entries.

ii) For the number of odd entries in row $n$ of the Pascal triangle we observe:

\begin{tabular}{|r||r|r|r|r|r|r|r|r|r|r|r|r|r|r|r|r|r|r|}
\hline$n$ & 0 & 1 & 2 & 3 & 4 & 5 & 6 & 7 & 8 & 9 & 10 & 11 & 12 & 13 & 14 & 15 & 16 & 17 \\
\hline odd & 1 & 2 & 2 & 4 & 2 & 4 & 4 & 8 & 2 & 4 & 4 & 8 & 4 & 8 & 8 & 16 & 2 & 4 \\
\hline
\end{tabular}


Table 4: Parity in the Pascal triangle.

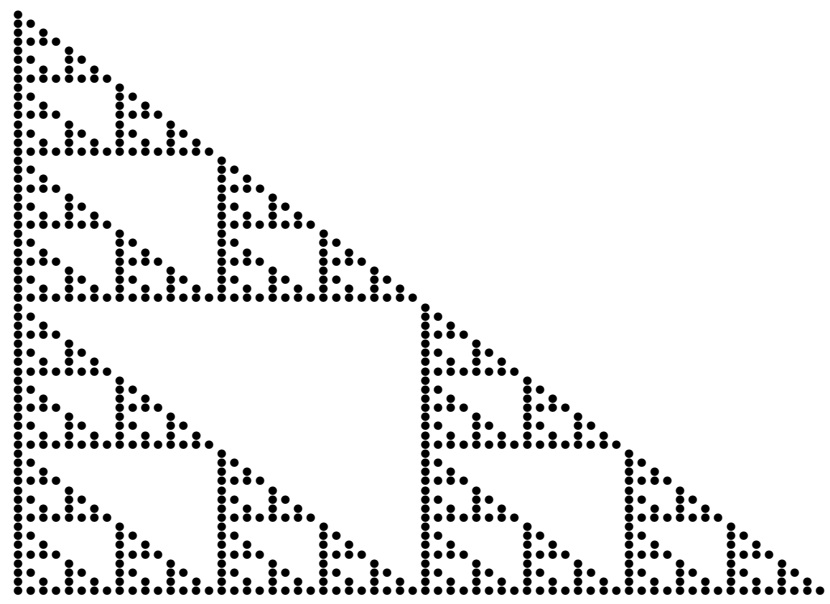

Letting again $n=\sum_{i=0}^{l} a_{i} \cdot 2^{i}$ be the 2-adic representation of $n$, the number of odd entries in row $n$ is given as $2^{w}$, where $w=w(n):=\sum_{i=0}^{l} a_{i} \in \mathbb{N}_{0}$ is the number of binary digits 1 in the 2-adic representation of $n$ :

We proceed by induction, the assertion being clear for $n=0$. By the repeating pattern described above, the number of odd entries in row $n \geq 1$ is twice the number of odd entries in row $n^{\prime}:=n-2^{l}=\sum_{i=0}^{l-1} a_{i} \cdot 2^{i}$. The latter is given as $2^{w\left(n^{\prime}\right)}$, where $w\left(n^{\prime}\right)=w(n)-1$, so that for row $n$ we get $2 \cdot 2^{w\left(n^{\prime}\right)}=2^{w}$. $\quad \sharp$

(0.4) The Sierpinski Sieve [1915]. Considering the Pascal triangles with $2^{i}$ rows, for $i \in \mathbb{N}_{0}$, and the repeating pattern of odd entries reveals a remarkable relationship to the following construction in fractal geometry. Actually, the construction of the sets $\mathcal{T}_{i}$ below shows that there is a natural bijection between the set of triangles in $\mathcal{T}_{i}$ and the odd entries in the Pascal triangle with $2^{i}$ rows:

We consider the open rectangular triangle $\mathcal{T}_{0}$ in the Euclidean plane with vertices $[0,0],[1,0]$ and $[0,1]$. We cut out the closed triangle with vertices $\left[\frac{1}{2}, \frac{1}{2}\right],\left[\frac{1}{2}, 0\right]$ and $\left[0, \frac{1}{2}\right]$, leaving a union $\mathcal{T}_{1}$ of three open triangles of halved edge lengths, each being a rescaled copy of $\mathcal{T}_{0}$; thus $\mathcal{T}_{1}$ covers $\frac{3}{4}$ of the area of $\mathcal{T}_{0}$. For each of the smaller triangles obtained we iterate this process, yielding an infinite descending chain $\mathcal{T}_{0} \supset \mathcal{T}_{1} \supset \mathcal{T}_{2} \supset \cdots$, where $\mathcal{T}_{i}$ consists of a total of $3^{i}$ triangles covering an area of $\frac{3^{i}}{2^{2 i+1}}$, for $i \in \mathbb{N}_{0}$. The limit $\mathcal{T}_{\infty}:=\bigcap_{i \geq 0} \mathcal{T}_{i}$ approached is called the fractal Sierpinski Sieve.

By construction, $\mathcal{T}_{\infty}$ is a measurable set of zero area, and we have $[y, x] \in \mathcal{T}_{\infty}$ whenever $[x, y] \in \mathcal{T}_{\infty}$. Moreover, letting $\mathcal{T}^{\prime} \subseteq[0,1]^{2}$ be the set of points $[x, y]$ such that either of $x, y, x+y$ equals $z \cdot 2^{-i}$, for some $i \in \mathbb{N}_{0}$ and $z \in \mathbb{N}_{0}$, we have $\mathcal{T}_{\infty} \cap \mathcal{T}^{\prime}=\emptyset$. (Starting with a closed triangle and removing open triangles 
instead, yields a larger, but less interesting fractal set next to $\mathcal{T}_{\infty}$ encompassing the points $[x, y] \in \mathcal{T}^{\prime}$ such that $x+y \leq 1$.) We derive a numerical description of the elements of $\mathcal{T}_{\infty}$, in particular showing that $\mathcal{T}_{\infty}$ is an infinite set:

Letting $\mathcal{T}_{i}^{\prime}:=\mathcal{T}_{i} \cap \mathcal{T}^{\prime}$ for $i \in \mathbb{N}_{0}$, we consider the map $\tau: \mathcal{T}_{0}^{\prime} \rightarrow[0,1]^{2}$ defined by

$$
\tau:[x, y] \mapsto\left\{\begin{aligned}
{[2 x, 2 y], } & \text { if } x<\frac{1}{2} \text { and } y<\frac{1}{2}, \\
{[2 x-1,2 y], } & \text { if } x>\frac{1}{2}, \\
{[2 x, 2 y-1], } & \text { if } y>\frac{1}{2} .
\end{aligned}\right.
$$

Note that $\tau\left(\mathcal{T}_{0}^{\prime}\right) \cap \mathcal{T}^{\prime}=\emptyset$; and recall that $[x, y] \in \mathcal{T}_{0}$ if and only if $x>0$, $y>0$ and $x+y<1$. Then, for $[x, y] \in \mathcal{T}_{0}^{\prime}$, we have $[x, y] \in \mathcal{T}_{1}^{\prime}$ if and only if $\tau([x, y]) \in \mathcal{T}_{0}^{\prime}$, and similarly $[x, y] \in \mathcal{T}_{i}^{\prime}$ if and only if $\tau([x, y]) \in \mathcal{T}_{i-1}^{\prime}$, for $i \in \mathbb{N}$, so that $[x, y] \in \mathcal{T}_{i}^{\prime}$ if and only if $\tau^{i}([x, y]) \in \mathcal{T}_{0}^{\prime}$. This implies that $[x, y] \in \mathcal{T}_{\infty}$ if and only if $\tau^{i}([x, y]) \in \mathcal{T}_{0}^{\prime}$ for all $i \in \mathbb{N}_{0}$.

Now, for $[x, y] \in \mathcal{T}_{0}^{\prime}$, the coordinates $x$ and $y$ have infinite 2-adic representation $x=\sum_{i>0} a_{i} \cdot 2^{-i}$ and $y=\sum_{i>0} b_{i} \cdot 2^{-i}$, respectively, where $a_{i}, b_{i} \in\{0,1\}$; note that the coordinates having finite 2-adic representations are captured in $\mathcal{T}^{\prime}$. Then in terms of 2-adic representations $\tau$ is given by shifting each of the given representations by one step and ignoring the first binary digit, that is $\left[x^{\prime}, y^{\prime}\right]:=\tau([x, y])$ is given as $x^{\prime}=\sum_{i>0} a_{i+1} \cdot 2^{-i}$ and $y^{\prime}=\sum_{i>0} b_{i+1} \cdot 2^{-i}$.

Hence if $[x, y] \in \mathcal{T}_{\infty}^{\prime}$ then, applying all powers of $\tau$ in turn, the condition $x+y<1$ implies that the case $a_{i}=b_{i}=1$ is excluded for all $i \in \mathbb{N}$, so that we have $a_{i}+b_{i} \leq 1$ for all $i \in \mathbb{N}$. Moreover, assume that $a_{i}+b_{i}=1$ for almost all $i \in \mathbb{N}$, then after applying a suitable power of $\tau$ we may assume that $a_{i}+b_{i}=1$ for all $i \in \mathbb{N}$, so that $x+y=1$, a contradiction. Hence we conclude that we additionally have $a_{i}=b_{i}=0$ for infinitely many $i \in \mathbb{N}$.

Conversely, let $[x, y] \in[0,1]^{2} \backslash \mathcal{T}^{\prime}$ be such that $a_{i}+b_{i} \leq 1$ for all $i \in \mathbb{N}$, and $a_{i}=b_{i}=0$ for infinitely many $i \in \mathbb{N}$. Then, after applying any power of $\tau$, there is $k \in \mathbb{N}$ such that $a_{i}+b_{i}=1$ for $i \in\{1, \ldots, k-1\}$, and $a_{k}=b_{k}=0$. This indeed implies that $x+y<1$; note that in view of the conditions imposed it is not necessary to require that $x+y$ has an infinite 2 -adic representation. $\sharp$

\section{Counting}

\section{Sets}

(1.1) Sets. a) A set is a collection of well-defined distinct objects forming a new entity [Cantor 1895]: Eine Menge ist eine gedankliche Zusammenfassung von bestimmten, wohlunterschiedenen Objekten der Anschauung oder des Denkens zu einem Ganzen. Hence we stick to naive set theory, but Russell's antinomy below shows that this generality leads to a contradiction.

The objects collected are called the elements of the set. For any set $M$ and any 
object $x$ either $x \in M$ or $x \notin M$ holds. Moreover, any set is uniquely determined by its elements, hence contains a particular element only once, and we disregard the order of the elements. The empty set $\emptyset=\{\}$ is the set without elements.

For example, there are the positive integers $\mathbb{N}:=\{1,2,3, \ldots\}$, the nonnegative integers $\mathbb{N}_{0}:=\{0,1,2, \ldots\}$, the integers $\mathbb{Z}:=\{0,1,-1,2,-2, \ldots\}$, the rational numbers $\mathbb{Q}$, the real numbers $\mathbb{R}$, the complex numbers $\mathbb{C}$.

Sets can be given by explicit enumeration or by description. The latter means that from a given set $M$ a new set $N$ is formed by giving a logical formula saying which of the elements of $M$ are elements of $N$ and which are not; for example, we have $\left\{x \in \mathbb{Z} ; x^{2}=1\right\}=\{1,-1\}$.

b) Russell's antinomy [1901]. By the generality of naive set theory, there is the set of all sets. Hence let $\mathcal{M}:=\{M$ set; $M \notin M\}$ be the set of all sets, which do not contain themselves as one of their elements, thus either $\mathcal{M} \in \mathcal{M}$ or $\mathcal{M} \notin \mathcal{M}$. Assume we have $\mathcal{M} \in \mathcal{M}$, then $\mathcal{M}$ does not contain $\mathcal{M}$ as one of its elements, hence $\mathcal{M} \notin \mathcal{M}$, a contradiction. Assume we have $\mathcal{M} \notin \mathcal{M}$, then $\mathcal{M}$ does contain $\mathcal{M}$ as one of its elements, hence $\mathcal{M} \in \mathcal{M}$, again a contradiction.

Hence the set of all sets cannot possibly exist. Thus we indeed have to impose restrictions on which objects we may collect to form a set.

(1.2) Elementary constructions. a) Let $M$ and $N$ be sets. If for all $x \in M$ we have $x \in N$ then $M$ is called a subset of $N$, and $N$ is called a superset of $M$; we write $M \subseteq N$. If $M \subseteq N$ and $M \neq N$ then $M$ is called a proper subset of $N$; we write $M \subset N$. We have $M=N$ if and only if $M \subseteq N$ and $N \subseteq M$, and we have $\emptyset \subseteq M$ and $M \subseteq M$. For example, we have $\mathbb{N} \subset \mathbb{N}_{0} \subset \mathbb{Z} \subset \mathbb{Q} \subset \mathbb{R} \subset \mathbb{C}$.

b) The sets $M \cap N:=\{x ; x \in M$ and $x \in N\}=\{x \in M ; x \in N\}=\{x \in N ; x \in$ $M\}$ and $M \backslash N:=\{x \in M ; x \notin N\}$ are called intersection and difference of $M$ and $N$, respectively. If $M \cap N=\emptyset$ then $M$ and $N$ are called disjoint. If $\mathcal{I} \neq \emptyset$ is a set, and $M_{i}$ is a set for $i \in \mathcal{I}$, then their intersection is defined as $\bigcap_{i \in \mathcal{I}} M_{i}:=\left\{x ; x \in M_{i}\right.$ for all $\left.i \in \mathcal{I}\right\}$.

Moreover, $M \cup N:=\{x ; x \in M$ or $x \in N\}$ is called the union of $M$ and $N$; if additionally $M \cap N=\emptyset$ then the union of $M$ and $N$ is called disjoint, written as $M \cup \dot{\cup} N$. If $\mathcal{I}$ is a set, and $M_{i}$ is a set for $i \in \mathcal{I}$, then their union is defined as $\bigcup_{i \in \mathcal{I}} M_{i}:=\left\{x ; x \in M_{i}\right.$ for some $\left.i \in \mathcal{I}\right\}$.

c) Let $\mathcal{P}(M):=\{L ; L \subseteq M\}$ be the power set of $M$; we have $\mathcal{P}(\emptyset)=\{\emptyset\}$.

d) For $x \in M$ and $y \in N$ let $[x, y]:=\{x,\{x, y\}\}$ be the associated ordered pair with first and second components $x$ and $y$, respectively; hence for $x \neq y$ we have $[x, y] \neq[y, x]$. Let $M \times N:=\{[x, y] ; x \in M, y \in N\}$ be the Cartesian product of $M$ and $N$; hence $M \times N \neq \emptyset$ if and only if both $M \neq \emptyset$ and $N \neq \emptyset$.

(1.3) Maps. Let $M$ and $N$ be sets. A relation between $M$ and $N$ is a subset $R \subseteq M \times N$, and $x \in M$ and $y \in N$ such that $[x, y] \in R$ are said to be related by $R$; we also write $x R y$. A relation $f \subseteq M \times N$ such that for all $x \in M$ there 
is a unique $y \in N$ satisfying $[x, y] \in f$ is called a map from $M$ to $N$; we write $f: M \rightarrow N: x \mapsto y=f(x)$, and also $[f(x) \in N ; x \in M]$, where the element $y$ is called the image of $x$, while $x$ is called a preimage of $y$.

The sets $M$ and $N$ are called the source and the domain of $f$, respectively. The set $\operatorname{im}(f):=\{y \in N ; y=f(x)$ for some $x \in M\}$ is called the image of $f$. For a subset $N^{\prime} \subseteq N$, the set $f^{-1}\left(N^{\prime}\right):=\left\{x \in M ; f(x) \in N^{\prime}\right\}$ is called the preimage of $N^{\prime}$, with respect to $f$. For a subset $M^{\prime} \subseteq M$, the restriction of $f$ to $M^{\prime}$ is defined as $\left.f\right|_{M^{\prime}}: M^{\prime} \rightarrow N: x \mapsto f(x)$.

The map $f: M \rightarrow N$ is called surjective if $\operatorname{im}(f)=N$, that is for all $y \in N$ there is some $x \in M$ such that $y=f(x)$. Moreover, $f$ is called injective if for all $y \in N$ the preimage $f^{-1}(y)$ has at most one element, that is for all $y \in N$ there is at most one element $x \in M$ such that $y=f(x)$, or equivalently we have $f(x) \neq f\left(x^{\prime}\right) \in N$ whenever $x \neq x^{\prime} \in M$. Finally, $f$ is called bijective if it is both surjective and injective, that is $f^{-1}(y)$ is a singleton set for all $y \in N$, or equivalently for all $y \in N$ there is a unique $x \in M$ such that $y=f(x)$.

The $\operatorname{map} \operatorname{id}_{M}: M \rightarrow M: x \mapsto x$ is called the identity map. The composition of maps $f: M \rightarrow N$ and $g: N \rightarrow U$, where $U$ is a set, is defined as $g f=g \cdot f=$ $g \circ f: M \rightarrow U: x \mapsto g(f(x))$. We have $f \cdot \operatorname{id}_{M}=f$ and $\operatorname{id}_{N} \cdot f=f$; moreover $g f$ is surjective whenever $f$ and $g$ are surjective, and $g f$ is injective whenever $f$ and $g$ are injective.

If $f: M \rightarrow N$ is bijective, the relation $f^{-1}:=\{[y, x] \in N \times M ;[x, y] \in f\}$ is a map as well, $f^{-1}: N \rightarrow M$ is called the inverse map of $f$. Hence we have $f\left(f^{-1}(y)\right)=y$ for all $y \in N$, thus $f f^{-1}=\operatorname{id}_{N}$, and $f^{-1}(f(x))=x$ for all $x \in M$, thus $f^{-1} f=\operatorname{id}_{M}$. Moreover, $f^{-1}$ is bijective such that $\left(f^{-1}\right)^{-1}=f$.

Let $\operatorname{Maps}(M, N):=\{f \subseteq M \times N ; f$ map $\}$. Moreover, let $\operatorname{Inj}(M, N):=\{f \in$ $\operatorname{Maps}(M, N) ; f$ injective $\}$ and $\operatorname{Surj}(M, N):=\{f \in \operatorname{Maps}(M, N) ; f$ surjective $\}$, as well as $\operatorname{Bij}(M, N):=\operatorname{Inj}(M, N) \cap \operatorname{Surj}(M, N)$.

(1.4) Dedekind-Peano axioms. a) A set $N$ fulfilling the following conditions is called a set of positive integers: There is an element $1 \in N$ and an injective successor map $N \rightarrow N \backslash\{1\}: n \mapsto n^{\prime}$, such that the principle of induction holds: For any subset $M \subseteq N$ such that $1 \in M$, and such that for any $n \in M$ we also have $n^{\prime} \in M$, we already have $M=N$.

The successor map is surjective as well, hence is bijective: Let $M:=\{1\} \dot{\cup} \operatorname{im}(')$, then by induction we have $M=N$.

b) The set $\mathbb{N}:=\{1,2, \ldots\}$ of positive integers together with the successor map $\mathbb{N} \rightarrow \mathbb{N} \backslash\{1\}: n \mapsto n+1$ fulfills the above conditions; we take the existence of $\mathbb{N}$ and its arithmetic properties for granted. The set $\mathbb{N}$ is the unique model of a set of positive integers $N$, that is there is a unique map $f: \mathbb{N} \rightarrow N$ fulfilling $f(1)=1$ and $f(n+1)=f(n)^{\prime}$ for all $n \in \mathbb{N}$, and $f$ is bijective:

Let $M \subseteq \mathbb{N}$ be the set of all $n \in \mathbb{N}$ such that there is a unique $f_{n}:\{1, \ldots, n\} \rightarrow N$ fulfilling $f_{n}(1)=1$ and $f_{n}(m+1)=f_{n}(m)^{\prime}$ for all $m<n$; hence we have 
$1 \in M$, and for $1 \neq n \in M$ we have $\left.f_{n}\right|_{\{1, \ldots, n-1\}}=f_{n-1}$. The surjectivity of the successor map of $\mathbb{N}$ implies that $f_{n}$ is uniquely extendible to an admissible map $f_{n+1}:\{1, \ldots, n+1\} \rightarrow N$, thus by induction for $\mathbb{N}$ we have $M=\mathbb{N}$.

We have $1 \in \operatorname{im}(f)$, and for $f(n) \in \operatorname{im}(f)$ we have $f(n)^{\prime}=f(n+1) \in \operatorname{im}(f)$, thus induction for $N$ yields $\operatorname{im}(f)=N$, that is $f$ is surjective. Let $M \subseteq \mathbb{N}$ be the set of all $n \in \mathbb{N}$ such that $f^{-1}(f(n))=\{n\}$, then $f(\mathbb{N} \backslash\{1\}) \subseteq N \backslash\{1\}$ implies $f^{-1}(1)=\{1\}$, and we have $f^{-1}(f(n+1))=f^{-1}\left(f(n)^{\prime}\right)=f^{-1}(f(n))+1=$ $\{n+1\}$ for $n \in \mathbb{N}$, thus induction for $\mathbb{N}$ yields $M=\mathbb{N}$, that is $f$ is injective. $\sharp$

(1.5) Cardinality. a) Let $N$ be a set. If there is $n \in \mathbb{N}_{0}$ such that there is a bijection $f:\{1, \ldots, n\} \rightarrow N$, where we let $\{1, \ldots, 0\}:=\emptyset$, then $N$ is called finite of cardinality $|N|:=n$; otherwise $N$ is called infinite, and we write $|N|=\infty$. For a finite set $N$ the cardinality is uniquely determined:

We may assume that $N=\{1, \ldots, n\}$, for some $n \in \mathbb{N}$, and there is a bijection $f:\{1, \ldots, m\} \rightarrow\{1, \ldots, n\}$ for some $m \in \mathbb{N}$ such that $m \leq n$. We proceed by induction on $n \in \mathbb{N}$ : If $n=1$, then $m=1$ as well. If $n \geq 2$, then letting $k:=f(m)$ we get a bijection $f:\{1, \ldots, m-1\} \rightarrow\{1, \ldots, k-1, k+1, \ldots, n\}$. Using the bijection $\{1, \ldots, n-1\} \rightarrow\{1, \ldots, k-1, k+1, \ldots, n\}$ defined by $i \mapsto i$ for $i \leq k-1$, and $i \mapsto i+1$ for $i \geq k$, we may assume that $f:\{1, \ldots, m-1\} \rightarrow$ $\{1, \ldots, n-1\}$ is a bijection as well, and by induction we have $n-1=m-1$. $\sharp$

If $N$ is a finite set and $M \subseteq N$, then we may assume that $M=\{1, \ldots, m\} \subseteq$ $\{1, \ldots, n\}=N$, where $n=|N|$, hence $M$ is finite as well and we have $|M| \leq|N|$, where $|M|=|N|$ if and only if $M=N$.

Moreover, a map $f: N \rightarrow N$ is injective if and only if $f$ is surjective: There is a subset $N^{\prime} \subseteq N$ such that $\left.f\right|_{N^{\prime}}: N^{\prime} \rightarrow \operatorname{im}(f)$ is a bijection; we have $N^{\prime}=N$ if and only if $f$ is injective, and from $\left|N^{\prime}\right|=|\operatorname{im}(f)| \leq|N|$ we conclude that $\left|N^{\prime}\right|=|N|$ if and only if $f$ is surjective. In particular, since $\mathbb{N}$ possesses an injective but not surjective successor map, we conclude that $\mathbb{N}$ is infinite.

b) Any set $N$ is infinite if and only if there is an injective map $\mathbb{N} \rightarrow N$ : If there is such a map, then we may assume that $\mathbb{N} \subseteq N$, and since $\mathbb{N}$ is infinite we infer that $N$ is infinite as well. Conversely, let now $N$ be infinite. Then naively we might want to proceed as follows: We choose $x_{1} \in N$, and then successively $x_{n+1} \in N \backslash\left\{x_{1}, \ldots, x_{n}\right\}$, since by assumption we have $N \backslash\left\{x_{1}, \ldots, x_{n}\right\} \neq \emptyset$ for all $n \in \mathbb{N}$, yielding the injective map $f: \mathbb{N} \rightarrow N: n \mapsto x_{n}$. But this is not justified, neither by the induction principle nor directly by the choice principle! Instead, we have to argue by transfinite induction as follows:

Assume to the contrary that there is no injective map $\mathbb{N} \rightarrow N$. We consider the set $\mathcal{F}:=\bigcup_{n \in \mathbb{N}_{0}} \operatorname{Inj}(\{1, \ldots, n\}, N)$; note that $\mathcal{F} \neq \emptyset$ since $\operatorname{Inj}(\emptyset, N) \neq \emptyset$. Denoting the upper bound of the source of $f \in \mathcal{F}$ by $n(f) \in \mathbb{N}_{0}$, the set $\mathcal{F}$ is partially ordered by letting $f \leq f^{\prime}$ whenever $n(f) \leq n\left(f^{\prime}\right)$ and $\left.f^{\prime}\right|_{\{1, \ldots, n(f)\}}=f$. If $f_{1} \leq$ $f_{2} \leq \cdots \leq f_{k} \leq \cdots$ is a chain in $\mathcal{F}$, then $f_{\infty}: M_{\infty}:=\bigcup_{k \in \mathbb{N}}\left\{1, \ldots, n\left(f_{k}\right)\right\} \rightarrow$ $N: i \mapsto f_{k}(i)$, whenever $i \leq n\left(f_{k}\right)$, is a well-defined map, and since for any $i, j \in M_{\infty}$ there is $k \in \mathbb{N}$ such that $i, j \leq n\left(f_{k}\right)$ we infer that $f_{\infty}$ is injective. By 
induction we have $M_{\infty}=\mathbb{N}$ or $M_{\infty}=\left\{1, \ldots, n\left(f_{\infty}\right)\right\}$ is finite. By assumption, the former case cannot occur, thus $f_{\infty} \in \mathcal{F}$ is an upper bound of the given chain. Thus by Zorn's Lemma, see (5.2), there is a maximal element $f_{0} \in \mathcal{F}$. Since $f_{0}:\left\{1, \ldots, n\left(f_{0}\right)\right\} \rightarrow N$ is not surjective, choosing $x_{0} \in N \backslash \operatorname{im}\left(f_{0}\right)$, we may extend $f_{0}$ to a map $\widetilde{f}_{0} \in \mathcal{F}$ by letting $\widetilde{f}_{0}\left(n\left(f_{0}\right)+1\right):=x_{0}$, contradicting the maximality of $f_{0} \in \mathcal{F}$.

c) Sets $M$ and $N$ are called equicardinal if there is a bijection $M \rightarrow N$. A set $N$ is called Dedekind infinite [1888] if there is a proper subset $M \subset N$ such that $M$ and $N$ are equicardinal, that is there is an injective map $f: N \rightarrow N$ which is not surjective. Hence any Dedekind infinite set is infinite; conversely, any infinite set $N$ is Dedekind infinite: We may assume $\mathbb{N} \subseteq N$, then $f: N \rightarrow N$ defined by $f(x)=x$ for $x \in N \backslash \mathbb{N}$, and $f(n):=n+1$ for $n \in \mathbb{N} \subseteq N$, is injective but not surjective.

(1.6) Basic counting principles. Let $M$ and $N$ be finite sets. Then we have the sum principle, saying that if $M \cap N=\emptyset$ then $|M \cup \dot{\cup} N|=|M|+|N|$ : Let $f:\{1, \ldots, m\} \rightarrow M=\left\{x_{1}, \ldots, x_{m}\right\}: i \mapsto x_{i}$ and $g:\{1, \ldots, n\} \rightarrow N=$ $\left\{y_{1}, \ldots, y_{n}\right\}: j \mapsto y_{j}$ be bijective, where $m:=|M| \in \mathbb{N}_{0}$ and $n:=|N| \in \mathbb{N}_{0}$. Then the map $h:\{1, \ldots, m+n\} \rightarrow M \cup \dot{\cup} N$ defined by $h(k)=x_{k}$ for $k \leq m$, and $h(k)=y_{k-m}$ for $k \geq m+1$, is a bijection.

Moreover, we have the product principle $|M \times N|=|M| \cdot|N|$ : The map $\{1, \ldots, m n\} \rightarrow M \times N:(i-1) n+j \mapsto\left[x_{i}, y_{j}\right]$ is a bijection; in other words we have $m$ and $n$ possibilities for the first and second components, respectively.

We have $|\operatorname{Maps}(M, N)|=|N|^{|M|}$ : For $m \in \mathbb{N}$ let $N^{m}:=N \times \cdots \times N$ be the $m$-fold Cartesian power of $N$, where $N^{1}$ can be identified with $N$; we let $N^{0}:=\{[]\}$, a singleton set. Then $\operatorname{Maps}(M, N) \rightarrow N^{m}: \alpha \mapsto\left[\alpha\left(x_{1}\right), \ldots, \alpha\left(x_{m}\right)\right]$ is a bijection, hence by induction on $m$ we get $|\operatorname{Maps}(M, N)|=n^{m}$.

In particular, we have $|\mathcal{P}(N)|=2^{|N|}$ : The map $\operatorname{Maps}(N,\{0,1\}) \rightarrow \mathcal{P}(N): f \mapsto$ $f^{-1}(1)$, is a bijection, implying $|\mathcal{P}(N)|=|\operatorname{Maps}(N,\{0,1\})|=2^{|N|}$; the elements of $\operatorname{Maps}(N,\{0,1\})$ are called the indicator maps of $N$.

\section{Selections}

(2.1) Selections without repetitions. Let $N$ be a finite set of cardinality $n:=|N| \in \mathbb{N}_{0}$, hence we may assume that $N=\{1, \ldots, n\}$, and let $k \in \mathbb{N}_{0}$. We discuss various ways of selecting from $N$, see Table 5 :

a) A $k$-arrangement or $k$-permutation of $N$ is an ordered selection without repetitions, that is an injective map $f: K:=\{1, \ldots, k\} \rightarrow N$. Let $\mathcal{S}_{k}(n):=\operatorname{Inj}(K, N)$; hence we have $\mathcal{S}_{k}(n)=\emptyset$ for $k>n$. For $k=n$ we get $\mathcal{S}_{n}:=\mathcal{S}_{n}(n)=\operatorname{Bij}(N, N)$, being called the permutations of $N$.

We show that for $k \leq n$ we have $\left|\mathcal{S}_{k}(n)\right|=n_{(k)}:=\prod_{i=0}^{k-1}(n-i) \in \mathbb{N}$, called a falling factorial, where we define the empty product as $n_{(0)}:=1$ : We proceed 
by induction on $k \in \mathbb{N}_{0}$, the case $k=0$ being trivial. For $k \geq 1$ we have $n$ possibilities to choose $f(k) \in N$, for any of these there are $\mid \operatorname{Inj}(\{1, \ldots, k-$ $1\}, N \backslash\{f(k)\})|=| \mathcal{S}_{k-1}(n-1) \mid$ possibilities for $f$ left, hence we get $\left|\mathcal{S}_{k}(n)\right|=$ $n \cdot\left|\mathcal{S}_{k-1}(n-1)\right|=n \cdot \prod_{i=0}^{k-2}(n-1-i)=n \cdot \prod_{i=1}^{k-1}(n-i)=\prod_{i=0}^{k-1}(n-i)$.

Since $n_{(k)}=0$ for $k>n$, we have $\left|\mathcal{S}_{k}(n)\right|=n_{(k)} \in \mathbb{N}_{0}$ for all $k \in \mathbb{N}_{0}$. Moreover, we have $\left|\mathcal{S}_{n}\right|=n !:=n_{(n)} \in \mathbb{N}$, called $n$-factorial; in particular we have $0 !:=1$. Using this, for $k \leq n$ we get $\left|\mathcal{S}_{k}(n)\right|=n_{(k)}=\frac{n !}{(n-k) !} \in \mathbb{N}$.

For example, if any of $k=4$ men marries one of $n=6$ women, then this can be done in $\frac{6 !}{2 !}=6 \cdot 5 \cdot 4 \cdot 3=360$ ways.

b) A $k$-subset or $k$-combination of $N$ is an unordered selection without repetitions, that is a subset $M \subseteq N$ such that $|M|=k$, which hence can be identified with the associated indicator map $N \rightarrow\{0,1\}$. Let $\mathcal{P}_{k}(n):=\{M \subseteq$ $N ;|M|=k\}$; hence we have $\mathcal{P}_{k}(n)=\emptyset$ for $k>n$.

We determine $\left|\mathcal{P}_{k}(n)\right| \in \mathbb{N}$ for $k \leq n$ : Any $k$-subset $M \subseteq N$ gives rise to $\left|\mathcal{S}_{k}\right|$ arrangements, that is injective maps $f:\{1, \ldots, k\} \rightarrow N$ such that $\operatorname{im}(f)=M$, hence we have $\left|\mathcal{S}_{k}(n)\right|=\left|\mathcal{S}_{k}\right| \cdot\left|\mathcal{P}_{k}(n)\right|$, yielding $\left|\mathcal{P}_{k}(n)\right|=\frac{n_{(k)}}{k !}=:\left(\begin{array}{l}n \\ k\end{array}\right) \in \mathbb{N}$, being called a binomial coefficient; note that it is not obvious at all that this is an integer, here it follows from its interpretation as a cardinality. Since $\frac{n_{(k)}}{k !}=0$ for $k>n$, we have $\left|\mathcal{P}_{k}(n)\right|=\left(\begin{array}{l}n \\ k\end{array}\right) \in \mathbb{N}_{0}$ for all $k \in \mathbb{N}_{0}$. Moreover, for $k \leq n$ we have $\left(\begin{array}{l}n \\ k\end{array}\right)=\frac{n !}{k !(n-k) !}=\left(\begin{array}{c}n \\ n-k\end{array}\right) \in \mathbb{N}$.

For example, in the lottery ' 6 -from-49', there are $k=6$ balls drawn from an urn containing $n=49$ distinguishable balls, hence there are $\left(\begin{array}{c}49 \\ 6\end{array}\right)=\frac{49}{6 \cdot 43}=$ $13983816 \sim 1.4 \cdot 10^{7}$ distinct draws.

(2.2) Selections with repetitions. We discuss further ways of selecting from $N=\{1, \ldots, n\}$, where $n \in \mathbb{N}_{0}$, and let $k \in \mathbb{N}_{0}$, see Table 5 :

a) A $k$-tuple of $N$ is an ordered selection with repetitions, that is a map $K:=\{1, \ldots, k\} \rightarrow N$, which hence can be identified with an element of the $k$-fold Cartesian power $N^{k}$ of $N$; we have $|\operatorname{Maps}(K, N)|=|N|^{|K|}=n^{k} \in \mathbb{N}$.

For example, in the football pool '13er-Wette', the outcome of $k=13$ soccer matches is encoded into a map $\{1, \ldots, 13\} \rightarrow\{0,1,2\}$, by identifying 'home team wins' with 1 , 'guest team wins' with 2 , and 'draw' with 0 ; hence we have $n=3$ and there are $3^{13}=1594323 \sim 1.6 \cdot 10^{6}$ distinct outcomes.

b) A $k$-multiset on $N$ is an unordered selection with repetitions, that is a map $\mu: N \rightarrow \mathbb{N}_{0}: i \mapsto \mu_{i}$, or equivalently an $n$-tuple $\mu=\left[\mu_{1}, \ldots, \mu_{n}\right] \in \mathbb{N}_{0}^{n}$, such that $\sum_{i=1}^{n} \mu_{i}=k$, where $\mu_{i} \in \mathbb{N}_{0}$ is called the associated multiplicity, and $|\mu|:=k$ is called the cardinality of $\mu$; we also write the multiset associated with $\mu$ as $1^{\mu_{1}} \cdots n^{\mu_{n}}$. In particular, the multiplicity-free multisets, that is we have $\mu_{i} \leq 1$ for all $i \in N$, can be identified with the $k$-subsets of $N$. Let $\mathcal{M}_{k}(n):=\left\{\left[\mu_{1}, \ldots, \mu_{n}\right] \in \mathbb{N}_{0}^{n} ; \sum_{i=1}^{n} \mu_{i}=k\right\}$; hence we have $\mathcal{M}_{0}(0)=\{[]\}$ and $\mathcal{M}_{k}(0)=\emptyset$ for $k \geq 1$. 
Table 5: Selections of an $n$-set.

\begin{tabular}{|r||c|c||c|}
\hline$k$-selections & without repetitions & with repetitions & of $k$-multisets \\
\hline \hline ordered & $n_{(k)}$ arrangements & $n^{k}$ tuples & $\left.\begin{array}{c}k \\
\mu_{1}, \ldots, \mu_{n}\end{array}\right)$ \\
\hline unordered & $\left(\begin{array}{l}n \\
k\end{array}\right)$ subsets & $\left(\begin{array}{c}n+k-1 \\
k\end{array}\right)$ multisets & 1 \\
\hline
\end{tabular}

We determine $\left|\mathcal{M}_{k}(n)\right| \in \mathbb{N}$ for $n \geq 1$ : Given $\mu=\left[\mu_{1}, \ldots, \mu_{n}\right] \in \mathcal{M}_{k}(n)$, writing the associated multiset as $[\bullet \ldots \bullet|\bullet \ldots \bullet| \ldots \mid \bullet \ldots \bullet]$, with $\mu_{j}$ entries ' $\bullet$ ' in the $j$-th slot, for $j \in\{1, \ldots, n\}$, and counting the entries 'I', we get a tuple of length $(n-1)+\sum_{j=1}^{n} \mu_{j}=n+k-1$, uniquely determining $\mu$ by the $(n-1)$-subset of the positions of the 'I'.

Formally, letting $\sigma(\mu):=\left\{\sum_{j=1}^{i}\left(\mu_{j}+1\right) \in \mathbb{N} ; i \in\{1, \ldots, n-1\}\right\}$, an $(n-1)$ subset of $\{1, \ldots, n+k-1\}$, yields an injective map $\sigma: \mathcal{M}_{k}(n) \rightarrow \mathcal{P}_{n-1}(n+k-1)$. Conversely, given a subset $\left\{s_{1}, \ldots, s_{n-1}\right\} \subseteq\{1, \ldots, n+k-1\}$, letting $\mu_{1}:=$ $s_{1}-1 \in \mathbb{N}_{0}$ as well as $\mu_{i}:=s_{i}-s_{i-1}-1 \in \mathbb{N}_{0}$ for $i \in\{2, \ldots, n-1\}$, and $\mu_{n}:=k-\sum_{i=1}^{n-1} \mu_{i}=k-s_{n-1}+(n-1) \geq(n+k-1)-(n+k-1)=0$, we have $\mu:=\left[\mu_{1}, \ldots, \mu_{n}\right] \in \mathcal{M}_{k}(n)$, and from $\sum_{j=1}^{i}\left(\mu_{j}+1\right)=s_{i}$, for $i \in\{1, \ldots, n-1\}$, we get $\sigma(\mu)=\left\{s_{1}, \ldots, s_{n-1}\right\}$. Hence $\sigma$ is surjective, thus is a bijection.

Either picture yields $\left|\mathcal{M}_{k}(n)\right|=\left|\mathcal{P}_{n-1}(n+k-1)\right|=\left(\begin{array}{c}n+k-1 \\ n-1\end{array}\right)=\left(\begin{array}{c}n+k-1 \\ k\end{array}\right) . \quad \sharp$

Note that the former formula is not defined for $n=0$, but the latter does hold for $n=0$ as well. For example, $\mathrm{I}^{4} \mathrm{MP}^{2} \mathrm{~S}^{4}$ is a multiset on the Latin alphabet $\{\mathrm{A}, \ldots, \mathrm{Z}\}$, hence we have $n=26$, and $\left[4_{\mathrm{l}}, 1_{\mathrm{M}}, 2_{\mathrm{P}}, 4_{\mathrm{S}}\right]$ has cardinality $k=4+$ $1+2+4=11$; the number of 11-multisets on the Latin alphabet is given as $\left(\begin{array}{l}36 \\ 11\end{array}\right)=600805296 \sim 6 \cdot 10^{8}$.

(2.3) Selections from multisets. We generalize the notion of selection without repetition to multisets. To this end, let $N:=\{1, \ldots, n\}$, where $n \in \mathbb{N}_{0}$, let $\mu=\left[\mu_{1}, \ldots, \mu_{n}\right] \in \mathcal{M}_{k}(n)$, where $k \in \mathbb{N}_{0}$, and let $l \in \mathbb{N}_{0}$, see Table 5 :

a) An $l$-arrangement or $l$-permutation of $\mu$ is an ordered selection of $\mu$, that is a map $f: L:=\{1, \ldots, l\} \rightarrow N$ such that $\left|f^{-1}(i)\right| \leq \mu_{i}$, for all $i \in N$; in particular, if $\mu$ is multiplicity-free describing the subset $M:=\left\{i \in N ; \mu_{i}=\right.$ $1\} \subseteq N$, we recover $\operatorname{Inj}(L, M)$. Hence we have $l \leq k$, and for $k=l$ we have $\left|f^{-1}(i)\right|=\mu_{i}$, for all $i \in N$; in the latter case $f$ is called a permutation of $\mu$.

We show that the number of permutations of $\mu$ is given by the multinomial coefficient $\left(\begin{array}{c}k \\ \mu_{1}, \ldots, \mu_{n}\end{array}\right):=\frac{k !}{\prod_{i=1}^{n} \mu_{i} !} \in \mathbb{N}$ : We proceed by induction on $n \in \mathbb{N}_{0}$. For $n=0$ we have $k=0$ as well, thus there is a unique map $f: \emptyset \rightarrow \emptyset$, where $\frac{0 !}{1}=1$. For $n \geq 1$ we have $\left(\begin{array}{c}k \\ \mu_{n}\end{array}\right)$ possibilities to choose $f^{-1}(n) \subseteq K:=\{1, \ldots, k\}$, for any of these there are $\left(\begin{array}{c}k-\mu_{n} \\ \mu_{1}, \ldots, \mu_{n-1}\end{array}\right)$ possibilities for $f: K \backslash f^{-1}(n) \rightarrow N \backslash\{n\}$ left, hence there are $\frac{k !}{\mu_{n} !\left(k-\mu_{n}\right) !} \cdot \frac{\left(k-\mu_{n}\right) !}{\prod_{i=1}^{n-1} \mu_{i} !}=\frac{k !}{\prod_{i=1}^{n} \mu_{i} !}$ possibilities for $f$. 
b) An $l$-submultiset of $\mu$ is an unordered selection of $\mu$, that is a map $\lambda=$ $\left[\lambda_{1}, \ldots, \lambda_{n}\right] \in \mathcal{M}_{l}(n)$ such that $\lambda_{i} \leq \mu_{i}$, for all $i \in N$. Hence we have $l \leq k$, and in the case $k=l$ we have $\lambda=\mu$. Moreover, summing over $l \in\{0, \ldots, k\}$ there are $\prod_{i=1}^{n}\left(\mu_{i}+1\right) \in \mathbb{N}$ submultisets of $\mu$.

For example, the multiset $I^{4} \mathrm{MP}^{2} \mathrm{~S}^{4}$ has $\left(\begin{array}{c}11 \\ 4,1,2,4\end{array}\right)=\frac{11 !}{4 ! \cdot 1 ! \cdot 2 ! \cdot 4 !}=34650$ permutations, for example IIIIMPPSSSS and MISSISSIPPI; moreover, IMS ${ }^{2}$ is the 4submultiset $\left[1_{\mathrm{I}}, 1_{\mathrm{M}}, 2_{\mathrm{S}}\right]$ of $\left[4_{\mathrm{I}}, 1_{\mathrm{M}}, 2_{\mathrm{P}}, 4_{\mathrm{S}}\right]$, allowing for the permutation MISS.

(2.4) Binomial coefficients. a) We generalize the notion of falling factorials and binomial coefficients. To this end, let $k \in \mathbb{N}_{0}$ and let $X$ be an indeterminate. Then the polynomials $X_{(k)}:=\prod_{i=0}^{k-1}(X-i) \in \mathbb{Z}[X]$ and $X^{(k)}:=\prod_{i=0}^{k-1}(X+$ i) $\in \mathbb{Z}[X]$ are called falling and rising factorials, respectively, where again $X_{(0)}=X^{(0)}:=1 \in \mathbb{Z}[X]$. Hence both $X_{(k)}$ and $X^{(k)}$ are monic of degree $k$, and we have $X^{(k)}=(X+k-1)_{(k)}$ as well as the reciprocity $(-X)_{(k)}=$ $\prod_{i=0}^{k-1}(-X-i)=(-1)^{k} \cdot \prod_{i=0}^{k-1}(X+i)=(-1)^{k} X^{(k)}$. Moreover, for any $z \in \mathbb{C}$ by evaluating we get the complex numbers $z_{(k)} \in \mathbb{C}$ and $z^{(k)} \in \mathbb{C}$.

Still having $k !=k_{(k)} \in \mathbb{N}$, we let $\left(\begin{array}{l}X \\ k\end{array}\right):=\frac{X_{(k)}}{k !} \in \mathbb{Q}[X]$, being called the associated binomial coefficient, thus $\left(\begin{array}{c}X \\ k\end{array}\right)$ has leading coefficient $\frac{1}{k !}$ and degree $k$, and we have the negation $\left(\begin{array}{c}-X \\ k\end{array}\right)=\frac{(-X)_{(k)}}{k !}=(-1)^{k} \cdot \frac{(X+k-1)_{(k)}}{k !}=(-1)^{k} \cdot\left(\begin{array}{c}X+k-1 \\ k\end{array}\right)$. For $k \neq 0$ we let $\left(\begin{array}{c}X \\ -k\end{array}\right):=0 \in \mathbb{Z}[X]$; note that $(-k)$ ! is not defined.

For any $z \in \mathbb{C}$ we get $\left(\begin{array}{c}z \\ k\end{array}\right)=\frac{z_{(k)}}{k !} \in \mathbb{C}$, while for $k \neq 0$ we have $\left(\begin{array}{c}z \\ -k\end{array}\right)=0 \in \mathbb{C}$. In particular we have the combinatorial reciprocity $\left|\mathcal{M}_{k}(n)\right|=\left(\begin{array}{c}n+k-1 \\ n-1\end{array}\right)=$ $\left(\begin{array}{c}n+k-1 \\ k\end{array}\right)=(-1)^{k} \cdot\left(\begin{array}{c}-n \\ k\end{array}\right)$, for all $n \in \mathbb{N}_{0}$, relating the number of $k$-multisets of $N:=\{1, \ldots, n\}$ to the number of $k$-subsets of $N$; this is elucidated in (12.3).

b) To explain the name 'binomial coefficient', let $X$ and $Y$ be indeterminates. Then $(X+Y)^{n}=\sum_{\mu \in \mathcal{M}_{n}(2)}\left(\begin{array}{c}n \\ \mu_{1}, \mu_{2}\end{array}\right) X^{\mu_{1}} Y^{\mu_{2}}=\sum_{k=0}^{n}\left(\begin{array}{l}n \\ k\end{array}\right) X^{k} Y^{n-k} \in \mathbb{Z}[X, Y]$ holds in the bivariate polynomial ring $\mathbb{Z}[X, Y]$, where $\left(\begin{array}{c}n \\ \mu_{1}, \mu_{2}\end{array}\right)$ is the number of permutations of the multiset $\mu \in \mathcal{M}_{n}(2)$. Hence $\left(\begin{array}{l}n \\ k\end{array}\right)$ appears in the expansion of the $n$-th power, where $n \in \mathbb{N}_{0}$, of the binomial $X+Y \in \mathbb{Z}[X, Y]$.

Hence evaluating at $y:=1$ yields $(X+1)^{n}=\sum_{k=0}^{n}\left(\begin{array}{l}n \\ k\end{array}\right) X^{k} \in \mathbb{Z}[X]$, thus evaluating further at $x:=1$ yields $\sum_{k=0}^{n}\left(\begin{array}{l}n \\ k\end{array}\right)=2^{n} \in \mathbb{C}$, while evaluating further at $x:=-1$ for $n \neq 0$ yields $\sum_{k=0}^{n}(-1)^{k}\left(\begin{array}{l}n \\ k\end{array}\right)=0 \in \mathbb{C}$.

To explain the name 'multinomial coefficient', let $X_{1}, \ldots, X_{n}$ be indeterminates, using the multinomial $\sum_{i=1}^{n} X_{i} \in \mathbb{Z}\left[X_{1}, \ldots, X_{n}\right]$ in the multivariate polynomial ring $\mathbb{Z}\left[X_{1}, \ldots, X_{n}\right]$, we get $\left(\sum_{i=1}^{n} X_{i}\right)^{k}=\sum_{\mu \in \mathcal{M}_{k}(n)}\left(\left(\begin{array}{c}k \\ \mu_{1}, \ldots, \mu_{n}\end{array}\right) \cdot \prod_{i=1}^{n} X_{i}^{\mu_{i}}\right)$.

(2.5) Identities for binomial coefficients. a) We show that for all $k \in \mathbb{Z}$ we have the triangle identity $\left(\begin{array}{l}X \\ k\end{array}\right)=\left(\begin{array}{c}X-1 \\ k-1\end{array}\right)+\left(\begin{array}{c}X-1 \\ k\end{array}\right) \in \mathbb{Q}[X]:$ If $k<0$ then both sides vanish, if $k=0$ then both sides are equal to the constant polynomial 1 . Hence we may assume that $k \geq 1$. Then both sides have degree $k$, hence their 
difference either vanishes or has at most $k$ complex zeroes. Thus it suffices to show that the above identity holds for all $x:=n \in \mathbb{N}$ :

Let $N:=\{1, \ldots, n\}$. Then we have $\mathcal{P}_{k}(n)=\{M \subseteq N ;|M|=k\}=\{M \subseteq$ $N ;|M|=k, n \in M\} \cup \dot{\cup}\{M \subseteq N ;|M|=k, n \notin M\}$, where $\{M \subseteq N ;|M|=$ $k, n \in M\} \rightarrow \mathcal{P}_{k-1}(n-1): M \mapsto(M \backslash\{n\})$ and $\{M \subseteq N ;|M|=k, n \notin M\} \rightarrow$ $\mathcal{P}_{k}(n-1): M \mapsto M$ are bijections, thus we get $\left(\begin{array}{l}n \\ k\end{array}\right)=\left|\mathcal{P}_{k}(n)\right|=\left|\mathcal{P}_{k-1}(n-1)\right|+$ $\left|\mathcal{P}_{k}(n-1)\right|=\left(\begin{array}{c}n-1 \\ k-1\end{array}\right)+\left(\begin{array}{c}n \\ k-1\end{array}\right)$.

b) We show that for all $k \in \mathbb{Z}$ we have the Vandermonde identity $\left(\begin{array}{c}X+Y \\ k\end{array}\right)=$ $\sum_{i=0}^{k}\left(\begin{array}{c}X \\ i\end{array}\right)\left(\begin{array}{c}Y \\ k-i\end{array}\right) \in \mathbb{Q}[X, Y]:$ If $k<0$ then the left hand side vanishes, while the right hand side is the empty sum. Hence we may assume that $k \geq 0$, then both sides have total degree $k$. If their difference does not vanish, then viewing it as an element of $\mathbb{Q}[X][Y]$, there are at most $k(k+1)$ complex roots of any of its at most $k+1$ non-vanishing coefficient polynomials in $\mathbb{Q}[X]$, which each have degree at most $k$, and evaluating at a complex number not in this set there are at most $k$ complex roots of the resulting non-vanishing polynomial in $\mathbb{Q}[Y]$. Thus it suffices to show that the above identity holds for all $[x, y]:=[m, n] \in \mathbb{N}_{0}^{2}$ :

Let $M$ and $N$ be disjoint sets such that $|M|=m$ and $|N|=n$, then we have $\left(\begin{array}{c}m+n \\ k\end{array}\right)=|\{L \subseteq M \dot{\cup} N ;|L|=k\}|=\coprod_{i=0}^{k}\{L \subseteq M \dot{\cup} N ;|L|=k,|L \cap M|=i\}$, where the condition $|L \cap M|=i$ is equivalent to saying $|L \cap N|=k-i$. Thus we get $|\{L \subseteq M \cup N ;|L|=k,|L \cap M|=i\}|=\left(\begin{array}{c}m \\ i\end{array}\right)\left(\begin{array}{c}n \\ k-i\end{array}\right)$, implying $|\{L \subseteq M \dot{\cup} N ;|L|=k\}|=\sum_{i=0}^{k}\left(\begin{array}{c}m \\ i\end{array}\right)\left(\begin{array}{c}n \\ k-i\end{array}\right)$.

(2.6) The Pascal triangle. Letting $k \in \mathbb{N}_{0}$, evaluating at $n \in \mathbb{N}_{0}$ the recursion $\left(\begin{array}{l}n \\ k\end{array}\right)=\left(\begin{array}{l}n-1 \\ k-1\end{array}\right)+\left(\begin{array}{c}n-1 \\ k\end{array}\right)$ gives rise to the Pascal triangle shown in Table 3 , allowing to compute binomial coefficients by using additions alone, but no multiplications. The Pascal triangle has a rich structure, where we point out only a few of its properties; note that these typically do not generalize to the polynomial case $\left(\begin{array}{l}X \\ k\end{array}\right)$ :

i) We have palindromicity $\left(\begin{array}{l}n \\ k\end{array}\right)=\left(\begin{array}{c}n \\ n-k\end{array}\right)$ : Since $\left(\begin{array}{l}n \\ k\end{array}\right)=0$ for $k>n$, and $\left(\begin{array}{c}n \\ -k\end{array}\right)=0$ for $k \neq 0$, we may assume $k \leq n$, where we observe $\left(\begin{array}{l}n \\ k\end{array}\right)=\frac{n !}{k !(n-k) !}=\left(\begin{array}{c}n \\ n-k\end{array}\right)$.

Alternatively, for $k \leq n$ there is the bijection $\mathcal{P}_{k}(n) \rightarrow \mathcal{P}_{n-k}(n): M \mapsto(N \backslash M)$.

ii) From $\mathcal{P}(n)=\coprod_{k=0}^{n} \mathcal{P}_{k}(n)$ we recover the row sum formula $\sum_{k=0}^{n}\left(\begin{array}{l}n \\ k\end{array}\right)=2^{n}$.

iii) For $n \geq 1$, the alternating row sum formula $\sum_{k=0}^{n}(-1)^{k}\left(\begin{array}{l}n \\ k\end{array}\right)=0$ is equivalent to $\left|\mathcal{P}_{\text {even }}(n)\right|=\sum_{0 \leq k \leq n \text { even }}\left(\begin{array}{l}n \\ k\end{array}\right)=\sum_{0 \leq k \leq n \text { odd }}\left(\begin{array}{l}n \\ k\end{array}\right)=\left|\mathcal{P}_{\text {odd }}(n)\right|$, where $\mathcal{P}_{\text {even }}(n):=\coprod_{0 \leq k \leq n \text { even }} \mathcal{P}_{k}(n)$ and $\mathcal{P}_{\text {odd }}(n):=\coprod_{0 \leq k \leq n \text { odd }} \mathcal{P}_{k}(n)$. This in turn in view of $|\mathcal{P}(n)|=2^{n}$ is equivalent to $\left|\mathcal{P}_{\text {even }}(n)\right|=2^{n-1}=\left|\mathcal{P}_{\text {odd }}(n)\right|$.

Alternatively, the latter can be shown by induction on $n \in \mathbb{N}$ as follows: For $n=1$ we have $\mathcal{P}_{\text {even }}(1)=\{\emptyset\}$ and $\mathcal{P}_{\text {odd }}(1)=\{\{1\}\}$, and for $n \geq 2$ letting $N:=\{1, \ldots, n\}$ we have $\mathcal{P}_{\text {even }}(n)=\{M \subseteq N ;|M|$ even, $n \in M\} \cup \dot{\cup}\{M \subseteq$ $N ;|M|$ even, $n \notin M\}$, where $\{M \subseteq N ;|M|$ even, $n \in M\} \rightarrow \mathcal{P}_{\text {odd }}(n-1): M \mapsto$ 
$(M \backslash\{n\})$ and $\{M \subseteq N ;|M|$ even, $n \notin M\} \rightarrow \mathcal{P}_{\text {even }}(n-1): M \mapsto M$ are bijections. Hence we have $\left|\mathcal{P}_{\text {even }}(n)\right|=\left|\mathcal{P}_{\text {odd }}(n-1)\right|+\left|\mathcal{P}_{\text {even }}(n-1)\right|=2^{n}$.

iv) We have the partial column sums $\sum_{i=0}^{n}\left(\begin{array}{l}i \\ k\end{array}\right)=\left(\begin{array}{l}n+1 \\ k+1\end{array}\right)$ : We proceed by induction on $n \in \mathbb{N}_{0}$, where for $n=0$ we have $\left(\begin{array}{l}0 \\ k\end{array}\right)=\left(\begin{array}{c}1 \\ k+1\end{array}\right)$, and for $n \geq 1$ we have $\sum_{i=0}^{n}\left(\begin{array}{l}i \\ k\end{array}\right)=\left(\begin{array}{l}n \\ k\end{array}\right)+\sum_{i=0}^{n-1}\left(\begin{array}{l}i \\ k\end{array}\right)=\left(\begin{array}{l}n \\ k\end{array}\right)+\left(\begin{array}{c}n \\ k+1\end{array}\right)=\left(\begin{array}{l}n+1 \\ k+1\end{array}\right)$.

Alternatively, we have $\mathcal{P}_{k+1}(n+1)=\coprod_{i=1}^{n+1}\{M \subseteq\{1, \ldots, n+1\} ;|M|=k+$ $1, \max (M)=i\}$, where $\{M \subseteq\{1, \ldots, n+1\} ;|M|=k+1, \max (M)=i\} \rightarrow$ $\mathcal{P}_{k}(i-1): M \mapsto(M \backslash\{i\})$ is a bijection, hence $\left(\begin{array}{l}n+1 \\ k+1\end{array}\right)=\sum_{i=1}^{n+1}\left(\begin{array}{c}i-1 \\ k\end{array}\right)=\sum_{i=0}^{n}\left(\begin{array}{l}i \\ k\end{array}\right)$.

v) For partial diagonal sums we have $\sum_{i=0}^{k}\left(\begin{array}{c}X+i \\ i\end{array}\right)=\left(\begin{array}{c}X+k+1 \\ k\end{array}\right) \in \mathbb{Q}[X]$ : We proceed by induction on $k \in \mathbb{N}_{0}$, where for $k=0$ we have $\left(\begin{array}{c}X+0 \\ 0\end{array}\right)=1=$ $\left(\begin{array}{c}X+1 \\ 0\end{array}\right) \in \mathbb{Z}[X]$, while for $k \geq 1$ we get $\sum_{i=0}^{k}\left(\begin{array}{c}X+i \\ i\end{array}\right)=\left(\begin{array}{c}X+k \\ k\end{array}\right)+\left(\sum_{i=0}^{k-1}\left(\begin{array}{c}X+i \\ i\end{array}\right)\right)=$ $\left(\begin{array}{c}X+k \\ k\end{array}\right)+\left(\begin{array}{c}X+k \\ k-1\end{array}\right)=\left(\begin{array}{c}X+k+1 \\ k\end{array}\right) \in \mathbb{Q}[X]$.

For partial alternating row sums using negation this yields $\sum_{i=0}^{k}(-1)^{i}\left(\begin{array}{c}X \\ i\end{array}\right)=$ $\sum_{i=0}^{k}\left(\begin{array}{c}-X+i-1 \\ i\end{array}\right)=\left(\begin{array}{c}(-X-1)+k+1 \\ k\end{array}\right)=\left(\begin{array}{c}-(X-1)+k-1 \\ k\end{array}\right)=(-1)^{k}\left(\begin{array}{c}X-1 \\ k\end{array}\right) \in \mathbb{Q}[X]$. Evaluating at $x:=n$ we get $\sum_{i=0}^{k}(-1)^{k-i}\left(\begin{array}{c}n \\ i\end{array}\right)=\left(\begin{array}{c}n-1 \\ k\end{array}\right)$; for $n=k$ we recover $\sum_{i=0}^{n}(-1)^{n-i}\left(\begin{array}{c}n \\ i\end{array}\right)=\left(\begin{array}{c}n-1 \\ n\end{array}\right)=\delta_{0, n}$, using the Kronecker symbol $\delta \in\{0,1\}$.

(2.7) Congruences for binomial coefficients. a) Let $k, n \in \mathbb{N}_{0}$, and let $p \in \mathbb{Z}$ be a prime. We consider the question of when $p \mid\left(\begin{array}{l}n \\ k\end{array}\right)$ : To this end, let $n=\sum_{i \geq 0} a_{i} p^{i}$ and $k=\sum_{i \geq 0} b_{i} p^{i}$ be the $p$-adic representations of $n$ and $k$, respectively, where $a_{i}, b_{i} \in\{0, \ldots, p-1\}$. Then we have the Lucas congruence [1878] saying that $\left(\begin{array}{l}n \\ k\end{array}\right) \equiv \prod_{i \geq 0}\left(\begin{array}{c}a_{i} \\ b_{i}\end{array}\right)(\bmod p)$ :

Since for $i \in\{0, \ldots, p\}$ we have $p X\left(\begin{array}{l}p \\ i\end{array}\right)=\frac{p !}{i !(p-i) !}$ if and only if $i \in\{0, p\}$, we have $(X+Y)^{p}=\sum_{i=0}^{p}\left(\begin{array}{c}p \\ i\end{array}\right) X^{i} Y^{p-i}=X^{p}+Y^{p} \in \mathbb{F}_{p}[X, Y]$, where $\mathbb{F}_{p} \cong \mathbb{Z} / p \mathbb{Z}$ is the finite field of order $p$, and where we identify an integer in $\mathbb{Z}$ with its natural image in $\mathbb{F}_{p}$. This yields $\sum_{l=0}^{n}\left(\begin{array}{l}n \\ l\end{array}\right) X^{l}=(X+1)^{n}=\prod_{i \geq 0}(X+1)^{a_{i} p^{i}}=$ $\prod_{i \geq 0}\left(X^{p^{i}}+1\right)^{a_{i}}=\prod_{i \geq 0}\left(\sum_{j=0}^{p-1}\left(\begin{array}{c}a_{i} \\ j\end{array}\right) X^{j p^{i}}\right) \in \mathbb{F}_{p}[X]$. The coefficient of $X^{k}=$ $\prod_{i \geq 0} X^{b_{i} p^{i}}$ in the latter polynomial equals $\prod_{i \geq 0}\left(\begin{array}{c}a_{i} \\ b_{i}\end{array}\right)$.

Hence we have $p \nmid\left(\begin{array}{l}n \\ k\end{array}\right)$ if and only if $p \nmid\left(\begin{array}{l}a_{i} \\ b_{i}\end{array}\right)$ for all $i \geq 0$. Since $a_{i} \leq p-1$ we have $p \nmid\left(\begin{array}{c}a_{i} \\ b_{i}\end{array}\right)=\frac{\left(a_{i}\right)_{\left(b_{i}\right)}}{b_{i} !}$ if and only if $b_{i} \leq a_{i}$. This implies that $p \nmid\left(\begin{array}{l}n \\ k\end{array}\right)$ if and only if $b_{i} \leq a_{i}$ for all $i \geq 0$, that is the $p$-adic expansion of $k$ is contained in the $p$-adic expansion of $n$.

In particular, for $p=2$, letting again $w(n):=\sum_{i \geq 0} a_{i} \in \mathbb{N}_{0}$ be the number of binary digits 1 in the 2 -adic representation of $n$, then there are $2^{w(n)}$ possible 2 -adic representations contained in the 2 -adic representation of $n$. Thus we recover the number of odd entries in row $n$ of the Pascal triangle as given in (0.3). Moreover, we have obtained a number theoretic description of where the odd entries are actually located. 
b) It follows from the Lucas congruence, that $\left(\begin{array}{c}p n \\ p k\end{array}\right) \equiv\left(\begin{array}{l}n \\ k\end{array}\right)(\bmod p)$. We show combinatorially that actually $\left(\begin{array}{c}p n \\ p k\end{array}\right) \equiv\left(\begin{array}{l}n \\ k\end{array}\right)\left(\bmod p^{2}\right)$ :

To this end, we consider a $(p \times n)$-square grid, in which $p k$ squares out of the $p n$ ones are chosen; this yields $\left(\begin{array}{c}p n \\ p k\end{array}\right)$ possibilities. We let the group $C_{p}^{n}$ act on these grids by cyclically permuting the rows independently, and we consider the associated orbits. Considering a fixed row of a choice, since $p$ is a prime, it is either fixed or yields an orbit of length $p$, where the former case occurs if and only if it is fully chosen or consists of unchosen squares only. Thus the orbit length of a choice is given as $p^{i}$, where $i$ is the number of mixed rows in the above sense. Now, there are $\left(\begin{array}{l}n \\ k\end{array}\right)$ choices entirely consisting of fully chosen rows, while for the other ones there are at least 2 mixed rows. Hence there are $\left(\begin{array}{l}p n \\ p k\end{array}\right)-\left(\begin{array}{l}n \\ k\end{array}\right)$ of the latter, all of which have orbit length divisible by $p^{2}$.

In the same vein, we show algebraically that $\left(\begin{array}{l}p n \\ p k\end{array}\right) \equiv\left(\begin{array}{l}n \\ k\end{array}\right)\left(\bmod p^{3}\right)$ for $p \geq 5$ :

We proceed keeping the above picture. If a choice contains at least 3 mixed rows, then its orbit has length divisible by $p^{3}$. Hence we only have to consider choices with precisely 2 mixed rows. Since in this case we have $k-1$ fully chosen rows, keeping the mixed rows fixed, this amounts to $\left(\begin{array}{c}2 p \\ p\end{array}\right)-2$ possibilities (all choices excluding the cases where either of the rows considered is fully chosen), so that we have to show that $\left(\begin{array}{c}2 p \\ p\end{array}\right) \equiv 2\left(\bmod p^{3}\right)$; note that this is just the case $n=2$ and $k=1$ of the claim:

The Vandermode identity yields $\left(\begin{array}{c}2 p \\ p\end{array}\right)-2=-2+\sum_{i=0}^{p}\left(\begin{array}{c}p \\ i\end{array}\right)\left(\begin{array}{c}p \\ p-i\end{array}\right)=\sum_{i=1}^{p-1}\left(\begin{array}{c}p \\ i\end{array}\right)^{2}=$ $p^{2} \cdot \sum_{i=1}^{p-1}\left(\frac{(p-1)_{(i-1)}}{i !}\right)^{2}$. Hence we show that $\sum_{i=1}^{p-1}\left(\frac{(p-1)_{(i-1)}}{i !}\right)^{2} \equiv 0(\bmod p)$ :

We have $\sum_{i=1}^{p-1}\left(\frac{(p-1)_{(i-1)}}{i !}\right)^{2} \equiv \sum_{i=1}^{p-1}\left(\frac{(i-1) !}{i !}\right)^{2} \equiv \sum_{i=1}^{p-1}\left(\frac{1}{i}\right)^{2} \equiv \sum_{i=1}^{p-1} i^{2}(\bmod p)$; recall that $\mathbb{Z}_{p}^{*}=\{1, \ldots, p-1\}$, so that this is well-defined indeed. Finally, we use the well-known identity $\sum_{i=1}^{n} i^{2}=\frac{1}{6} n(n+1)(2 n+1)$, which is immediately proved by induction; see also (4.3). From this, for $p \geq 5$ we indeed get $\sum_{i=1}^{p-1} i^{2} \equiv$ $\frac{1}{6} p(p-1)(2 p-1) \equiv 0(\bmod p)$.

The statement does not hold for $p \leq 3$ : For $p=2$ we get $\left(\begin{array}{l}4 \\ 2\end{array}\right) \equiv 6 \not \equiv 2(\bmod 8)$ and for $p=3$ we get $\left(\begin{array}{l}6 \\ 3\end{array}\right) \equiv 20 \not \equiv 2(\bmod 27)$.

\section{Partitions and permutations}

(3.1) Partitions of sets. a) We discuss ways of forming partitions of $N:=$ $\{1, \ldots, n\}$, where $n \in \mathbb{N}_{0}$; hence let $k \in \mathbb{N}_{0}$ : A $k$-composition of $N$ is an ordered decomposition $\left[N_{1}, \ldots, N_{k}\right]$ of $N=\coprod_{i=1}^{k} N_{i}$ into $k$ pairwise disjoint blocks $N_{i} \neq \emptyset$. A $k$-partition of $N$ is an unordered decomposition of $N=$ $\coprod_{i=1}^{k} N_{i}$ into $k$ pairwise disjoint blocks $N_{i} \neq \emptyset$. For example, for $n=3$ the 2-partitions of $N$ are given as $N=\{1,2\} \dot{\cup}\{3\}=\{1,3\} \dot{\cup}\{2\}=\{2,3\} \dot{\cup}\{1\}$, hence the 2-compositions of $N$ are given as

$$
[\{1,2\},\{3\}],[\{3\},\{1,2\}],[\{1,3\},\{2\}],[\{2\},\{1,3\}],[\{2,3\},\{1\}],[\{1\},\{2,3\}] .
$$


Table 6: The Stirling triangle of the second kind.

\begin{tabular}{|r||rrrrrrrr|}
\hline$n \backslash k$ & 0 & 1 & 2 & 3 & 4 & 5 & 6 & 7 \\
\hline \hline 0 & 1 & & & & & & & \\
1 & 0 & 1 & & & & & & \\
2 & 0 & 1 & 1 & & & & & \\
3 & 0 & 1 & 3 & 1 & & & & \\
4 & 0 & 1 & 7 & 6 & 1 & & & \\
5 & 0 & 1 & 15 & 25 & 10 & 1 & & \\
6 & 0 & 1 & 31 & 90 & 65 & 15 & 1 & \\
7 & 0 & 1 & 63 & 301 & 350 & 140 & 21 & 1 \\
\hline
\end{tabular}

b) The number $S_{n, k} \in \mathbb{N}_{0}$ of $k$-partitions of $N$ is called the associated Stirling number of the second kind. Since any $k$-partition of $N$ gives rise to $k$ ! compositions of $N$, the number of $k$-compositions of $N$ is given as $k ! \cdot S_{n, k}$.

We have $S_{n, k}=0$ for $k>n$, and $S_{0,0}=1$. For $n \geq 1$ we have $S_{n, 0}=0$ and $S_{n, 1}=S_{n, n}=1$; since any 2-partition consists of a non-empty subset and its non-empty complement, we have $S_{n, 2}=\frac{2^{n}-2}{2}=2^{n-1}-1$; and since any $(n-1)$ partition consists of a 2-block and $n-1$ singleton blocks, we have $S_{n, n-1}=\left(\begin{array}{l}n \\ 2\end{array}\right)$. The number $B_{n}:=\sum_{k=0}^{n} S_{n, k} \in \mathbb{N}$, that is the number of all partitions of $N$, is called the associated Bell number.

For $n, k \geq 1$ we have the recursion $S_{n, k}=S_{n-1, k-1}+k \cdot S_{n-1, k}$, giving rise to the Stirling triangle of the second kind shown in Table 6 : Considering the $k$-partitions of $N$, we distinguish the cases whether or not the block containing $n$ is a singleton set; hence any $k$-partition of $N$ is obtained from either a $(k-1)$ partition of $N \backslash\{n\}$ by adding the $k$-th block $\{n\}$, or from a $k$-partition of $N \backslash\{n\}$ by adding $n$ to either of the $k$ blocks.

c) Letting $K:=\{1, \ldots, k\}$, we have already shown that $|\operatorname{Maps}(K, N)|=n^{k}$ and $|\operatorname{Inj}(K, N)|=n_{(k)}$. To determine the cardinality $|\operatorname{Surj}(K, N)|$ we argue as follows: Any map $f \in \operatorname{Maps}(K, N)$ is uniquely determined by the preimages $f^{-1}(1), \ldots, f^{-1}(n) \subseteq K$, where $f^{-1}(i) \neq \emptyset$ if and only if $i \in \operatorname{im}(f)$. Hence letting $f \mapsto\left[f^{-1}(1), \ldots, f^{-1}(n)\right]$ yields a bijection $\operatorname{from} \operatorname{Surj}(K, N)$ to the set of $n$-compositions of $K$. Thus we have $|\operatorname{Surj}(K, N)|=n ! \cdot S_{k, n}$.

Moreover, from $\operatorname{Maps}(K, N)=\bigsqcup_{M \subseteq N} \operatorname{Surj}(K, M)$ we get $n^{k}=|\operatorname{Maps}(K, N)|=$ $\sum_{m=0}^{n} \sum_{M \subseteq N,|M|=m}|\operatorname{Surj}(K, M)|=\sum_{m=0}^{n}\left(\begin{array}{l}n \\ m\end{array}\right) \cdot m ! \cdot S_{k, m}=\sum_{m=0}^{n} S_{k, m} n_{(m)}$. Since $S_{k, m}=0$ for $m>k$ this yields $n^{k}=\sum_{m=0}^{k} S_{k, m} n_{(m)}$ for all $n \in \mathbb{N}_{0}$, implying that $X^{k}=\sum_{m=0}^{k} S_{k, m} X_{(m)} \in \mathbb{Z}[X]$, relating powers, falling factorials and Stirling numbers of the second kind.

(3.2) Partitions of numbers. a) A $k$-composition of $n \in \mathbb{N}_{0}$, where $k \in \mathbb{N}_{0}$, is an ordered sum $n=\sum_{i=1}^{k} \lambda_{i}$ with $k$ parts $\lambda:=\left[\lambda_{1}, \ldots, \lambda_{k}\right] \in \mathbb{N}^{k}$; we write 
$|\lambda|:=n$, and only $n=0$ has a 0 -composition, namely [].

For $n \geq 1$ and $k \geq 1$ we may write a $k$-composition of $n$ as a tuple $[\bullet \ldots \bullet \mid$ $\bullet \ldots \bullet|\ldots| \bullet \cdots \bullet]$, with $\lambda_{i}$ entries ' $\bullet$ ' in the $i$-th slot, for $i \in\{1, \ldots, k\}$, that is with $n$ entries ' $\bullet$ ' and $k-1$ entries 'I' such that precisely $k-1$ of the $n-1$ spaces between adjacent ' $\bullet$ ' are filled with a ' $\mid$ '. Formally, letting $\lambda=\left[\lambda_{1}, \ldots, \lambda_{k}\right] \mapsto\left[\lambda_{1}-1, \ldots, \lambda_{k}-1\right]$ yields a bijection from the set of $k$ compositions of $n$ to the set $\mathcal{M}_{n-k}(k)$, hence the number of $k$-compositions of $n$ equals $\left|\mathcal{M}_{n-k}(k)\right|=\left(\begin{array}{c}n-1 \\ k-1\end{array}\right)$. For example, for $n=3$ the 2-compositions are given as $[\bullet \mid \bullet \bullet]$ and $[\bullet \bullet \mid \bullet]$, that is $3=1+2=2+1$.

Hence for $n \geq 1$ summation over $k$ shows that in total there are $\sum_{k=1}^{n}\left(\begin{array}{c}n-1 \\ k-1\end{array}\right)=$ $2^{n-1}$ compositions of $n$; combinatorially, starting from the $n$-tuple $[\bullet \ldots \bullet]$ there are $n-1$ spaces to be filled, yielding $2^{n-1}$ possibilities.

b) A $k$-partition of $n$ is an unordered $\operatorname{sum} n=\sum_{i=1}^{k} \lambda_{i}$ with $k$ parts $\lambda_{i} \in \mathbb{N}$. Hence we may assume that the parts are in non-increasing order $\lambda_{1} \geq \cdots \lambda_{k} \geq 1$, and we write $\lambda:=\left[\lambda_{1}, \ldots, \lambda_{k}\right] \vdash n=:|\lambda|$ and $l(\lambda):=k$, where in turn $|\lambda|$ and $l(\lambda)$ are called the size and the length of $\lambda$, respectively. The partition $\lambda$ can be identified with the set $\left\{[i, j] \in \mathbb{N}^{2} ; i \in\{1, \ldots, k\}, j \in\left\{1, \ldots, \lambda_{i}\right\}\right\}$, which is typically depicted by a Young diagram, that is rectangular array of boxes consisting of $l(\lambda)$ rows, where row $i$ contains $\lambda_{i}$ boxes. Letting $a_{i}(\lambda):=\mid\{j \in$ $\left.\{1, \ldots, k\} ; \lambda_{j}=i\right\} \mid \in \mathbb{N}_{0}$ be the multiplicity of $i$ as a part of $\lambda$, we also write $\lambda=\left[n^{a_{n}(\lambda)}, \ldots, 1^{a_{1}(\lambda)}\right]$; we have $\sum_{i=1}^{n} i a_{i}(\lambda)=n$ and $\sum_{i=1}^{n} a_{i}(\lambda)=k$, hence in particular $\left[a_{1}(\lambda), \ldots, a_{n}(\lambda)\right] \in \mathcal{M}_{k}(n)$. For example, all partitions of $n=3$, ordered reversed lexicographically, are given as $\left\{[3],[2,1],\left[1^{3}\right]\right\}$.

Let $P_{k}(n):=\left\{\lambda \in \mathbb{N}^{k} ; \lambda \vdash n\right\}$ be the set of all $k$-partitions of $n$, let $P_{\leq k}(n):=$ $\coprod_{i=0}^{k} P_{i}(n)$ be the set of all partitions of $n$ with at most $k$ parts, and let $P(n)=$ $\coprod_{k \geq 0} P_{k}(n)$ be the set of all partitions of $n$. Hence we have $P_{k}(n)=\emptyset$ for $k>n$, implying $P(n)=P_{\leq n}(n)=\coprod_{k=0}^{n} P_{k}(n)$. We have $P_{0}(0)=\{[]\}$, and $P_{0}(n)=\emptyset$ and $P_{1}(n)=\{[n]\}$ and $P_{2}(n)=\left\{[n-j, j] ; j \in\left\{1, \ldots,\left\lfloor\frac{n}{2}\right\rfloor\right\}\right\}$ and $P_{n-1}(n)=\left\{\left[2,1^{n-2}\right]\right\}$ and $P_{n}(n)=\left\{\left[1^{n}\right]\right\}$ for $n \geq 1$.

Let $p_{n, k}:=\left|P_{k}(n)\right| \in \mathbb{N}_{0}$ and $p_{n, \leq k}:=\sum_{i=0}^{k} p_{n, i}=\left|P_{\leq k}(n)\right| \in \mathbb{N}_{0}$ and $p_{n}:=$ $p_{n, \leq n}=\sum_{k \geq 0} p_{n, k}=|P(n)| \in \mathbb{N}$, where $p_{n, k}=0$ for $k>n$. For $n \geq k \geq 1$ we have the recursion $p_{n, k}=p_{n-1, k-1}+p_{n-k, k}$ : Considering the $k$-partitions $\lambda \vdash n$, we distinguish the cases $\lambda_{k}=1$ and $\lambda_{k} \geq 2$; hence any such $\lambda$ is obtained either from a $(k-1)$-partition $\left[\lambda_{1}, \ldots, \lambda_{k-1}\right] \vdash n-1$ as $\lambda=\left[\mu_{1}, \ldots, \mu_{k-1}, 1\right] \vdash n$, or from a $k$-partition $\left[\lambda_{1}, \ldots, \lambda_{k}\right] \vdash n-k$ as $\lambda=\left[\lambda_{1}+1, \ldots, \lambda_{k}+1\right] \vdash n$.

This yields $p_{n, k}=p_{n-k, \leq k}$ for $n \geq k \geq 0$ : Proceeding by induction on $n \in \mathbb{N}_{0}$, where the case $n=0$ as well as the case $k=0$ are trivial, for $n \geq k \geq 1$ we get $p_{n, k}=p_{n-1, k-1}+p_{n-k, k}=\left(\sum_{i=0}^{k-1} p_{n-k, i}\right)+p_{n-k, k}=\sum_{i=0}^{k} p_{n-k, i}=$ $p_{n-k, \leq k}$. Alternatively, the map $\left[\lambda_{1}, \ldots, \lambda_{i}\right] \mapsto\left[\lambda_{1}+1, \ldots, \lambda_{i}+1,1, \ldots, 1\right]$ yields a bijection $P_{\leq k}(n-k) \rightarrow P_{k}(n)$.

Fixing $n$, summation yields $p_{n}=\sum_{k=0}^{n} p_{n, k}$, which hence can be computed using the above recursion. The asymptotic behavior of $p_{n}$ is given by the Hardy- 
Ramanujan formula [1918] as $p_{n} \sim \frac{\exp \left(\pi \sqrt{\frac{2 n}{3}}\right)}{4 n \sqrt{3}}$. The asymptotic behavior of $p_{n, k}$ will be discussed in (4.4). For example we have:

\begin{tabular}{|r||r|r|r|r|r|r|r|r|r|r|r|r|r|r|r|r|}
\hline$n$ & 0 & 1 & 2 & 3 & 4 & 5 & 6 & 7 & 8 & 9 & 10 & 11 & 12 & 13 & 14 & 15 \\
\hline$p_{n}$ & 1 & 1 & 2 & 3 & 5 & 7 & 11 & 15 & 22 & 30 & 42 & 56 & 77 & 101 & 135 & 176 \\
\hline \hline$p_{n, 2}$ & 0 & 0 & 1 & 1 & 2 & 2 & 3 & 3 & 4 & 4 & 5 & 5 & 6 & 6 & 7 & 7 \\
\hline$p_{n, 3}$ & 0 & 0 & 0 & 1 & 1 & 2 & 3 & 4 & 5 & 7 & 8 & 10 & 12 & 14 & 16 & 19 \\
\hline
\end{tabular}

(3.3) Permutations. a) Let $n \in \mathbb{N}_{0}$. We consider the set of permutations $\mathcal{S}_{n}:=\operatorname{Bij}(N, N)$ of $N:=\{1, \ldots, n\}$, which with respect to concatenation of maps becomes a group, with identity element $\mathrm{id}_{N}$ and inverses given by inverses of maps, called the symmetric group on $N$. Writing permutations as tuples, we have $\mathcal{S}_{0}=\{[]\}$ and $\mathcal{S}_{1}=\{[1]\}$, and using lexicographic ordering we get $\mathcal{S}_{2}=\{[1,2],[2,1]\}$ and $\mathcal{S}_{3}=\{[1,2,3],[1,3,2],[2,1,3],[2,3,1],[3,1,2],[3,2,1]\}$.

Any permutation $\pi \in \mathcal{S}_{n}$ can be written as a product of disjoint cycles: We consider the directed graph with vertex set $\{1, \ldots, n\}$ having an edge $i \rightarrow j$ if $\pi(i)=j$. Since $\pi$ is a map, from any vertex precisely one edge emanates; since $\pi$ is surjective, at any vertex at least one edge ends, since $\pi$ is injective, at any vertex at most one edge ends, thus at any vertex precisely one edge ends. Hence the connected components of this graph are directed circles, showing that the cycle decomposition of $\pi$ is unique up to reordering; the number of vertices in a cycle is called its length; for a more formal description see (15.2).

Hence a permutation is described by its cycle decomposition, which is unique up to ordering and rotating the cycles; typically fixed points, that is cycles of length 1 , are left out. Note that inverses are given by reading cycles backwardly. In the standard cycle representation cycles are ordered from right to left with increasing smallest elements, the latter being chosen as starting points. For example, we have $\pi:=[11,10,1,7,9,2,5,3,4,6,8]=$ $(4,7,5,9)(2,10,6)(1,11,8,3) \in \mathcal{S}_{11}$ and $\pi^{-1}=(4,9,5,7)(2,6,10)(1,3,8,11)$.

b) Proceeding even further, given $\pi \in \mathcal{S}_{n}$ in standard cycle representation, leaving out the pairs of parentheses ')(' yields a sequence of numbers $((\pi))$, which can be considered as an element of $\mathcal{S}_{n}$ again. Conversely, given such a sequence of numbers, inserting a pair of parentheses ')(' right to any successive absolute left-to-right minimum yields a permutation in standard cycle representation.

Moreover, by this insertion algorithm we recover $\pi \in \mathcal{S}_{n}$ from $((\pi))$, showing that the map $\mathcal{S}_{n} \rightarrow \mathcal{S}_{n}: \pi \rightarrow((\pi))$ is injective, hence bijective, with inverse given by the insertion algorithm; note that hence $\pi \in \mathcal{S}_{n}$ has $k \in \mathbb{N}_{0}$ cycles if and only if $((\pi))$ has precisely $k$ successive minima. For example, $\pi$ as above yields $((\pi))=((\underline{4}, 7,5,9, \underline{2}, 10,6, \underline{1}, 11,8,3))$, having the successive minima as indicated, hence the insertion algorithm recovers $(4,7,5,9)(2,10,6)(1,11,8,3)$.

c) Given $\pi \in \mathcal{S}_{n}$, for $i \in \mathbb{N}$ let $a_{i}(\pi) \in \mathbb{N}_{0}$ be the number of cycles of $\pi$ of length $i$. Hence $k(\pi)=\sum_{i=1}^{n} a_{i}(\pi) \in \mathbb{N}_{0}$ is the number of cycles of $\pi$, and we have $\sum_{i=1}^{n} i a_{i}(\pi)=n$. Hence $\lambda(\pi):=\left[n^{a_{n}(\pi)}, \ldots, 1^{a_{1}(\pi)}\right] \in P_{k(\pi)}(n)$ is a partition of 
Table 7: Cycle types in $\mathcal{S}_{5}$.

\begin{tabular}{|l|ll|}
\hline$\lambda$ & & \\
\hline \hline$[5]$ & $\frac{5 !}{5}=24$ \\
\hline$[4,1]$ & $\frac{5 !}{4}=30$ \\
{$[3,2]$} & $\frac{5 !}{3 \cdot 2}=20$ \\
\hline
\end{tabular}

\begin{tabular}{|l|ll|}
\hline$\lambda$ & & \\
\hline \hline$\left[3,1^{2}\right]$ & $\frac{5 !}{2 ! \cdot 3}=20$ \\
{$\left[2^{2}, 1\right]$} & $\frac{5 !}{2 ! \cdot 2^{2}}=15$ \\
\hline$\left[2,1^{3}\right]$ & $\frac{5 !}{3 ! \cdot 2}=10$ \\
\hline$\left[1^{5}\right]$ & $\frac{5 !}{5 !}=1$ \\
\hline
\end{tabular}

$n$ with $k(\pi) \in \mathbb{N}_{0}$ parts, being called the cycle type of $\pi$. Note that if $\alpha \in \mathcal{S}_{n}$ then the cycle types of $\pi$ and $\alpha \pi \alpha^{-1}$ coincide: If $\left(i, \pi(i), \ldots, \pi^{l-1}(i)\right)$ is a cycle of $\pi$, for some $i \in\{1, \ldots, n\}$ and $l \in \mathbb{N}$, then $\left(\alpha(i), \alpha \pi(i), \ldots, \alpha \pi^{l-1}(i)\right)$ is a cycle of $\alpha \pi \alpha^{-1}$.

Given $\lambda=\left[n^{a_{n}}, \ldots, 1^{a_{1}}\right] \in P(n)$, there are $\frac{n !}{\prod_{i=1}^{n} a_{i} ! \cdot i^{a_{i}}}$ permutations in $\mathcal{S}_{n}$ with cycle type $\lambda$ : Consider the pattern $(\cdots)(\cdots) \cdots(\cdots)$ consisting of $a_{i} \in \mathbb{N}_{0}$ cycles of length $i$, for $i \in\{1, \ldots, n\}$, written in order of non-increasing lengths. There are $n$ ! possibilities to fill in pairwise distinct entries from $\{1, \ldots, n\}$. Permuting the cycles of the same lengths amongst themselves, and rotating any of the cycles, we thus get any permutation $\left(\prod_{i=1}^{n} a_{i} !\right) \cdot\left(\prod_{i=1}^{n} i^{a_{i}}\right)$ times.

(3.4) Permutations with a fixed number of cycles. a) Let $n \in \mathbb{N}_{0}$ and $k \in \mathbb{N}_{0}$. Then the number $s_{n, k}:=\left|\left\{\pi \in \mathcal{S}_{n} ; k(\pi)=k\right\}\right| \in \mathbb{N}_{0}$ of permutations in $\mathcal{S}_{n}$ having $k$ cycles is called the associated (signless) Stirling number of the first kind. Hence we have $s_{n, k}=\sum_{\lambda \in P_{k}(n)} \frac{n !}{\prod_{i=1}^{n} a_{i}(\lambda) ! \cdot i^{a_{i}(\lambda)}}$; for example, for $n=5$ we get the figures shown in Table 7 . Thus we have $s_{n, k}=0$ for $k>n$, and $s_{0,0}=1$. For $n \geq 1$ we have $s_{n, 0}=0$ and $s_{n, n}=1$, as well as $s_{n, 1}=(n-1)$ ! and $s_{n, n-1}=\left(\begin{array}{c}n \\ 2\end{array}\right)$; summing over $k$ yields $\sum_{k=0}^{n} s_{n, k}=\left|\mathcal{S}_{n}\right|=n$ !.

For $n, k \geq 1$ we have the recursion $s_{n, k}=s_{n-1, k-1}+(n-1) \cdot s_{n-1, k}$, giving rise to the Stirling triangle of the first kind shown in Table 8: Considering the permutations of $N$ having $k$ cycles, we distinguish the cases whether or not $n$ is a fixed point; hence any such permutation is obtained from either a permutation of $N \backslash\{n\}$ having $k-1$ cycles by adding the cycle $(n)$, or from a permutation of $N \backslash\{n\}$ having $k$ cycles by inserting $n$ into any of the $n-1$ positions in there.

This yields $\frac{s_{n, k}}{(n-1) !}=\sum_{m=0}^{n-1} \frac{s_{m, k-1}}{m !}$, for $n, k \geq 1$ : We proceed by induction on $n \in$ $\mathbb{N}$; the assertion being true for $n=1$, for $n \geq 2$ the recursion $s_{n, k}=s_{n-1, k-1}+$ $(n-1) \cdot s_{n-1, k}$ yields $\frac{s_{n, k}}{(n-1) !}=\frac{s_{n-1, k-1}}{(n-1) !}+\frac{s_{n-1, k}}{(n-2) !}=\frac{s_{n-1, k-1}}{(n-1) !}+\sum_{m=0}^{n-2} \frac{s_{m, k-1}}{m !}=$ $\sum_{m=0}^{n-1} \frac{s_{m, k-1}}{m !}$. In particular, for $k=2$ and $n \geq 1$ we get $s_{n, 2}=(n-1) ! \cdot h_{n-1}$, where $h_{n}:=\sum_{i=1}^{n} \frac{1}{i} \in \mathbb{Q}$ is the $n$-th harmonic number.

b) We have $X^{(n)}=\sum_{k=0}^{n} s_{n, k} X^{k} \in \mathbb{Z}[X]$ : By induction on $n \in \mathbb{N}_{0}$, where for $n \leq 1$ both sides are equal to $1 \in \mathbb{Z}[X]$, respectively $X \in \mathbb{Z}[X]$, we for $n \geq 2$ get 
Table 8: The Stirling triangle of the first kind.

\begin{tabular}{|r||rrrrrrrr|}
\hline$n \backslash k$ & 0 & 1 & 2 & 3 & 4 & 5 & 6 & 7 \\
\hline \hline 0 & 1 & & & & & & & \\
1 & 0 & 1 & & & & & & \\
2 & 0 & 1 & 1 & & & & & \\
3 & 0 & 2 & 3 & 1 & & & & \\
4 & 0 & 6 & 11 & 6 & 1 & & & \\
5 & 0 & 24 & 50 & 35 & 10 & 1 & & \\
6 & 0 & 120 & 274 & 225 & 85 & 15 & 1 & \\
7 & 0 & 720 & 1764 & 1624 & 735 & 175 & 21 & 1 \\
\hline
\end{tabular}

$X^{(n)}=(X+n-1) X^{(n-1)}=(X+n-1) \cdot \sum_{k=1}^{n-1} s_{n-1, k} X^{k}=\sum_{k=2}^{n} s_{n-1, k-1} X^{k}+$ $\sum_{k=1}^{n-1}(n-1) s_{n-1, k} X^{k}=\sum_{k=1}^{n}\left(s_{n-1, k-1}+(n-1) s_{n-1, k}\right) X^{k}=\sum_{k=1}^{n} s_{n, k} X^{k}$.

The reciprocity $X_{(n)}=(-1)^{n}(-X)^{(n)}$ yields $X_{(n)}=(-1)^{n} \cdot \sum_{k=0}^{n} s_{n, k}(-X)^{k}=$ $\sum_{k=0}^{n}(-1)^{n-k} s_{n, k} X^{k} \in \mathbb{Z}[X]$. This yields the following relationship between both kinds of Stirling numbers: Letting $\mathbb{Z}[X]_{\leq n}:=\{f \in \mathbb{Z}[X] \backslash\{0\} ; \operatorname{deg}(f) \leq$ $n\} \cup\{0\}$, then both $\left\{X^{k} ; k \in\{0, \ldots, n\}\right\}$ and $\left\{X_{(k)} ; k \in\{0, \ldots, n\}\right\}$ are $\mathbb{Z}$-bases of $\mathbb{Z}[X]_{\leq n}$, and thus from $X^{n}=\sum_{k=0}^{n} S_{n, k} X_{(k)} \in \mathbb{Z}[X]$ we infer $\left[S_{n, k}\right]_{n, k}^{-1}=$ $\left[(-1)^{n-k} s_{n, k}\right]_{n, k} \in \mathrm{GL}_{n+1}(\mathbb{Z})$.

(3.5) Permutations without fixed points. A permutation $\pi \in \mathcal{S}_{n}$, where $n \in \mathbb{N}_{0}$, having no fixed points, that is $\pi(i) \neq i$ for all $i \in\{1, \ldots, n\}$, is called a derangement. Let $\mathcal{D}(n) \subseteq \mathcal{S}_{n}$ be the set of all derangements, and let $D_{n}:=|\mathcal{D}(n)| \in \mathbb{N}_{0}$; we have $D_{0}=1$ and $D_{1}=0$ and $D_{2}=1$ and $D_{3}=2$.

For $n \geq 2$ we have the recursion $D_{n}=(n-1)\left(D_{n-2}+D_{n-1}\right)$ : For $\pi \in \mathcal{D}(n)$ we consider the cycle $(1, i, \ldots)$ of $\pi$ containing the letter 1 , where $i \in\{2, \ldots, n\}$, and we distinguish the cases whether this cycle has length 2 or $\geq 3$. In the first case, deleting the cycle $(1, i)$ from $\pi$, and leaving the other cycles unchanged, yields a derangement permuting the $n-2$ letters $\{2, \ldots, n\} \backslash\{i\}$; in the second case replacing the cycle $(1, i, j, \ldots)$ of $\pi$, where $j \in\{2, \ldots, n\} \backslash\{i\}$, by $(1, j, \ldots)$ yields a derangement permuting the $n-1$ letters $\{1, \ldots, n\} \backslash\{i\}$.

We derive a closed formula for $D_{n}$, also called the problème des rencontres: The above recursion can be rewritten as $D_{n}-n D_{n-1}=-\left(D_{n-1}-(n-1) D_{n-2}\right)$, for $n \geq 2$. Hence by induction we get $D_{n}-n D_{n-1}=(-1)^{n-1} \cdot\left(D_{1}-D_{0}\right)=$ $(-1)^{n}$, for $n \geq 1$. Considering the proportion $\frac{|\mathcal{D}(n)|}{\left|\mathcal{S}_{n}\right|}=\frac{D_{n}}{n !}$ this yields $\frac{D_{n}}{n !}-$ $\frac{D_{n-1}}{(n-1) !}=\frac{(-1)^{n}}{n !}$, thus $\frac{D_{n}}{n !}-1=\sum_{k=1}^{n}\left(\frac{D_{k}}{k !}-\frac{D_{k-1}}{(k-1) !}\right)=\sum_{k=1}^{n} \frac{(-1)^{k}}{k !}$. Hence we get $\frac{D_{n}}{n !}=\sum_{k=0}^{n} \frac{(-1)^{k}}{k !}$, for $n \in \mathbb{N}_{0}$; for more conceptual proofs see (4.5) and (10.1).

We have $\sum_{k \geq 0} \frac{(-1)^{k}}{k !}:=\lim _{n \rightarrow \infty}\left(\sum_{k=0}^{n} \frac{(-1)^{k}}{k !}\right)=\frac{1}{e} \sim 0.367879$, hence the pro- 
portion of derangements amongst all permutations is approximated by $\frac{D_{3}}{3 !}=$ $\frac{1}{3}<\frac{D_{n}}{n !}<\frac{3}{8}=\frac{D_{4}}{4 !}$, for $n \geq 5$; recall that $\frac{D_{0}}{0 !}=1$ and $\frac{D_{1}}{1 !}=0$ and $\frac{D_{2}}{2 !}=\frac{1}{2}$.

\section{Difference calculus}

(4.1) Discrete differentiation. a) We consider the set of all infinite sequences $\left[a_{0}, a_{1}, \ldots\right] \subseteq \mathbb{Q}$, that is the $\mathbb{Q}$-vector space $\mathcal{F}:=\operatorname{Maps}\left(\mathbb{N}_{0}, \mathbb{Q}\right)$. Since $\mathbb{N}_{0}$ is infinite, the polynomial ring $\mathbb{Q}[X]$ can be identified with the $\mathbb{Q}$-subspace $\left\{\mathbb{N}_{0} \rightarrow\right.$ $\mathbb{Q}: n \mapsto f(n) ; f \in \mathbb{Q}[X]\} \leq \mathcal{F}$ of polynomial maps. Similarly, $\mathbb{Q}(X)^{\circ}:=$ $\left\{\frac{f}{g} \in \mathbb{Q}(X) ; g(n) \neq 0\right.$ for all $\left.n \in \mathbb{N}_{0}\right\}$ can be identified with the $\mathbb{Q}$-subspace of $\mathcal{F}$ of rational maps. Let $\mathbb{Q}[X]_{\leq k}:=\{f \in \mathbb{Q}[X] \backslash\{0\} ; \operatorname{deg}(f) \leq k\} \dot{\cup}\{0\}$ for $k \geq 0$, and $\mathbb{Q}[X]_{\leq-k}:=\{0\}$ for $k \geq 1$; in particular, $\mathbb{Q}[X]_{\leq 0}=\mathbb{Q}$ consists of the constant maps.

We consider various maps on $\mathcal{F}$ : Let $\tau \in \operatorname{End}_{\mathbb{Q}}(\mathcal{F})$ be the shift operator defined by $\tau f: \mathbb{N}_{0} \rightarrow \mathbb{Q}: n \mapsto f(n+1)$. Let $\delta:=\tau-$ id $\in \operatorname{End}_{\mathbb{Q}}(\mathcal{F})$ be the difference operator, that is $\delta f: \mathbb{N}_{0} \rightarrow \mathbb{Q}: n \mapsto f(n+1)-f(n)$, being called the discrete derivative of $f$, which is the discrete analogue of differentiation; note that $\operatorname{ker}(\delta)=\mathbb{Q} \leq \mathcal{F}$.

Hence $\tau$ and $\delta$ restrict to $\mathbb{Q}$-linear maps on $\mathbb{Q}[X]$ given by $(\tau f)(X):=f(X+1)$ and $(\delta f)(X):=f(X+1)-f(X)$, respectively. Moreover, by letting $\tau\left(\frac{f}{g}\right):=\frac{\tau f}{\tau g}$, for $f, g \in \mathbb{Q}[X]$ such that $g \neq 0$, we obtain $\mathbb{Q}$-linear extensions of $\tau$ and $\delta$ to $\mathbb{Q}(X)$, which in turn restrict to $\mathbb{Q}(X)^{\circ}$.

We get a discrete analogue of the product rule as follows: For $f, g \in \mathcal{F}$ we have $\delta(f g): n \mapsto f(n+1) g(n+1)-f(n) g(n)=f(n+1) g(n+1)-f(n) g(n+1)+$ $f(n) g(n+1)-f(n) g(n)=(\delta f)(n) \cdot g(n+1)+f(n) \cdot(\delta g)(n)$, for $n \in \mathbb{N}_{0}$, hence $\delta(f g)=\delta f \cdot \tau g+f \cdot \delta g \in \mathcal{F}$.

b) Let $f \in \mathcal{F}$. Then for $i \geq 0$ we have $\delta^{i} f=(\tau-\text { id })^{i} f=\sum_{j=0}^{i}(-1)^{i-j}\left(\begin{array}{c}i \\ j\end{array}\right) \cdot \tau^{j} f \in$ $\mathcal{F}$. Thus $\left(\delta^{i} f\right)(0)$ is given in terms of values of $f$ as $\left(\delta^{i} f\right)(0)=\sum_{j=0}^{i}(-1)^{i-j}\left(\begin{array}{l}i \\ j\end{array}\right)$. $f(j)$; in particular, we have $\left(\delta^{i} f\right)(0) \in \mathbb{Z}$ whenever $f(\{0, \ldots, i\}) \subseteq \mathbb{Z}$.

Conversely, we get a discrete analogue of Taylor expansions: For $n \geq 0$ we have $\tau^{n}=(\delta+\mathrm{id})^{n}$, thus we get the Newton expansion $f(n)=\left(\tau^{n} f\right)(0)=$ $\left((\delta+\mathrm{id})^{n} f\right)(0)=\sum_{i=0}^{n}\left(\begin{array}{c}n \\ i\end{array}\right) \cdot\left(\delta^{i} f\right)(0)$, expressing the values of $f$ in terms of the derivatives $\left(\delta^{i} f\right)(0)$, and thus inverting the above formula.

For example, for the number of derangements in $\mathcal{S}_{n}$, where $n \in \mathbb{N}_{0}$, we already know that $D_{n}=\sum_{k=0}^{n}(-1)^{k} \cdot \frac{n !}{k !}=\sum_{k=0}^{n}(-1)^{k}\left(\begin{array}{l}n \\ k\end{array}\right) \cdot(n-k) !=\sum_{k=0}^{n}(-1)^{n-k}\left(\begin{array}{l}n \\ k\end{array}\right)$. $k$ !, which in present terms says that $D_{n}=\left(\delta^{n}(k \mapsto k !)\right)(0)$. Thus Newton expansion yields $n !=\sum_{i=0}^{n}\left(\begin{array}{c}n \\ i\end{array}\right) \cdot D_{i}$; for a combinatorial proof see (4.5).

(4.2) Polynomial functions. a) We look for polynomials providing the discrete analogue of the differentiation behavior of the power maps: To this end, we generalize the falling factorial $X_{(k)} \in \mathbb{Q}[X]_{\leq k}$, which so far is defined for 
$k \geq 0$, by letting $X_{(-k)}:=\frac{1}{\prod_{i=1}^{k}(X+i)} \in \mathbb{Q}(X)^{\circ}$, for $k \geq 1$.

Then we have $X_{(k)}=X_{(k-1)} \cdot(X-k+1) \in \mathbb{Q}(X)^{\circ}$ and $(X+1)_{(k)}=(X+$ $1) \cdot X_{(k-1)} \in \mathbb{Q}(X)^{\circ}$, for all $k \in \mathbb{Z}$ : This is immediate for $k \geq 1$, for $k=0$ we have $X_{(0)}=1=X_{(-1)} \cdot(X+1)$ and $(X+1)_{(0)}=1=(X+1) \cdot X_{(-1)}$, while for $k \geq 1$ we have $X_{(-k)}=\frac{1}{\prod_{i=1}^{k}(X+i)}=\frac{X+k+1}{\prod_{i=1}^{k+1}(X+i)}=X_{(-k-1)} \cdot(X-(-k)+1)$ and $(X+1)_{(-k)}=\frac{1}{\prod_{i=1}^{k}(X+i+1)}=\frac{X+1}{\prod_{i=1}^{k+1}(X+i)}=(X+1) \cdot X_{(-k-1)}$.

Thus we indeed have $\delta\left(X_{(k)}\right)=(X+1)_{(k)}-X_{(k)}=(X+1) \cdot X_{(k-1)}-X_{(k-1)}$. $(X-k+1)=k X_{(k-1)} \in \mathbb{Q}(X)^{\circ}$, for all $k \in \mathbb{Z}$. Hence for polynomial binomial coefficients we get $\delta\left(\begin{array}{c}X \\ k\end{array}\right)=\frac{1}{k !} \cdot \delta\left(X_{(k)}\right)=\frac{1}{(k-1) !} \cdot \delta\left(X_{(k-1)}\right)=\left(\begin{array}{c}X \\ k-1\end{array}\right) \in \mathbb{Q}[X]$, for $k \geq 1$; we have $\delta\left(\begin{array}{c}X \\ 0\end{array}\right)=\delta(1)=0 \in \mathbb{Q}[X]$. Moreover, for $k \geq 0$ we have $\delta\left(X_{(k)}\right) \in$ $\mathbb{Q}[X]_{\leq k-1} \backslash \mathbb{Q}[X]_{\leq k-2}$, hence $\delta$ induces a surjection $\mathbb{Q}[X]_{\leq k} \rightarrow \mathbb{Q}[X]_{\leq k-1}$, and we have $\delta^{-1}\left(\mathbb{Q}[X]_{\leq k-1}\right)=\mathbb{Q}[X]_{\leq k} \subseteq \mathcal{F}$. In particular, given $f \in \mathcal{F}$ and $k \geq 0$, we have $f \in \mathbb{Q}[X]_{\leq k}$ if and only if $\delta^{k+1}(f)=0$.

b) For $f \in \mathbb{Q}[X]_{\leq k}$, where $k \geq 0$, Newton expansion becomes $f=\sum_{i=0}^{k}\left(\delta^{i} f\right)(0)$. $\left(\begin{array}{c}X \\ i\end{array}\right)=\sum_{i=0}^{k} \frac{\left(\delta^{i} f\right)(0)}{i !} \cdot X_{(i)} \in \mathbb{Q}[X]_{\leq k}$ : Since both sides are polynomials of degree at most $k$, it suffices to show that the identity holds for all $x:=n \in\{0, \ldots, k\}$; since $\left(\begin{array}{c}n \\ i\end{array}\right)=0$ whenever $n<i$, we get $f(n)=\sum_{i=0}^{k}\left(\delta^{i} f\right)(0) \cdot\left(\begin{array}{c}n \\ i\end{array}\right)=\sum_{i=0}^{n}\left(\delta^{i} f\right)(0)$. $\left(\begin{array}{l}n \\ i\end{array}\right)$, which holds by Newton expansion.

Hence we have proved Newton's Theorem: Given a polynomial $f \in \mathbb{Q}[X]_{\leq k}$, we have $f\left(\mathbb{N}_{0}\right) \subseteq \mathbb{Z}$ if and only if $\left(\delta^{i} f\right)(0) \in \mathbb{Z}$ for all $i \in\{0, \ldots, k\}$; thus the $\mathbb{Z}$ submodule $\left\{f \in \mathbb{Q}[X]_{\leq k} ; f\left(\mathbb{N}_{0}\right) \subseteq \mathbb{Z}\right\} \leq \mathcal{F}$ is $\mathbb{Z}$-free with $\mathbb{Z}$-basis $\left\{\left(\begin{array}{c}X \\ 0\end{array}\right), \ldots,\left(\begin{array}{c}X \\ k\end{array}\right)\right\}$.

For example, for $X^{n} \in \mathbb{Q}[X]$ this yields a sum formula for the Stirling numbers of the second kind: From $X^{n}=\sum_{k=0}^{n} S_{n, k} X_{(k)} \in \mathbb{Z}[X]$, for $n \in \mathbb{N}_{0}$, we for $k \in \mathbb{N}_{0}$ get $k ! \cdot S_{n, k}=\left(\delta^{k} X^{n}\right)(0)=\left(\sum_{i=0}^{k}(-1)^{k-i}\left(\begin{array}{c}k \\ i\end{array}\right)(X+i)^{n}\right)(0)=\sum_{i=0}^{k}(-1)^{k-i}\left(\begin{array}{c}k \\ i\end{array}\right) i^{n}$; other proofs are given in (10.1) and (13.4).

(4.3) Discrete integration. a) Let $\mathcal{F}:=\operatorname{Maps}\left(\mathbb{N}_{0}, \mathbb{Q}\right)$, and let $\sigma \in \operatorname{End}_{\mathbb{Q}}(\mathcal{F})$ be the sum operator given by $\sigma f: \mathbb{N}_{0} \rightarrow \mathbb{Q}: n \mapsto \sum_{i=0}^{n-1} f(i)$, where $(\sigma f)(0)=$ 0 , which is the discrete analogue of integration with lower bound 0 .

Thus for $f \in \mathcal{F}$ and $n \in \mathbb{N}_{0}$ we have $(\delta \sigma f)(n)=\sum_{i=0}^{n} f(i)-\sum_{i=0}^{n-1} f(i)=$ $f(n)$, showing that $\delta \sigma=$ id, which is the discrete analogue of the theorem on integration-differentiation. Hence $\delta^{-1}(f)=\sigma f+\mathbb{Q} \subseteq \mathcal{F}$ are the discrete stem functions of $f$, where $\sigma f$ is the unique one such that $(\sigma f)(0)=0$.

The other way around we get $(\sigma \delta f)(n)=\sum_{i=0}^{n-1}(\delta f)(i)=\sum_{i=0}^{n-1}(f(i+1)-$ $f(i))=f(n)-f(0)$, that is $\sigma \delta f=f-f(0) \in \mathcal{F}$, which is the discrete analogue of the theorem on differentiation-integration; hence to determine the sum $\sum_{i=a}^{b} g(i)$, for $g \in \mathcal{F}$ and $a, b \in \mathbb{N}_{0}$, we may just evaluate any stem function $f \in \mathcal{F}$ of $g$, yielding $\sum_{i=a}^{b} g(i)=(\sigma g)(b+1)-(\sigma g)(a)=f(b+1)-f(a)$.

b) For example, from $\delta\left(X_{(k)}\right)=k X_{(k-1)} \in \mathbb{Q}(X)$, for $k \in \mathbb{Z}$, we get the 
stem functions $\sigma\left(X_{(k)}\right)=\frac{1}{k+1} \cdot X_{(k+1)} \in \mathbb{Q}[X]$ for $k \geq 0$, and $\sigma\left(X_{(k)}\right)=$ $\frac{1}{k+1} \cdot\left(X_{(k+1)}-\frac{1}{(-k-1) !}\right) \in \mathbb{Q}(X)^{\circ}$ for $k \leq-2$. But for $k=-1$ we get the discrete analogue of the logarithm $\sigma\left(X_{(-1)}\right): \mathbb{N}_{0} \rightarrow \mathbb{Q}: n \mapsto \sum_{i=0}^{n-1} \frac{1}{i+1}=h_{n}$, where $h_{n}$ is the $n$-th harmonic number, and $h_{0}:=0$.

In order to find the discrete analogue of the exponential map, we observe that $f \in \mathcal{F}$ fulfills $\delta f=f$ if and only if $f(n)=f(n+1)-f(n)$, that is $f(n+1)=2 f(n)$ for all $n \in \mathbb{N}_{0}$, which is equivalent to $f: \mathbb{N}_{0} \rightarrow \mathbb{Q}: n \mapsto c \cdot 2^{n}$, for some $c \in \mathbb{Q}$.

For example, we determine the power sums $s_{k}: \mathbb{N}_{0} \rightarrow \mathbb{N}_{0}: n \mapsto \sum_{i=0}^{n-1} i^{k}$, where $k \in \mathbb{N}_{0}$ : We have $s_{k}=\sigma\left(X^{k}\right)=\sum_{i=0}^{k} S_{k, i} \sigma\left(X_{(i)}\right)=\sum_{i=0}^{k} \frac{S_{k, i}}{i+1} \cdot X_{(i+1)} \in \mathbb{Q}[X]$, being a polynomial map of degree $k+1$ with leading coefficient $\frac{1}{k+1}$ and constant coefficient 0; in particular, we have $s_{0}=X \in \mathbb{Q}[X]$ and $s_{1}=\frac{1}{2} X(X-1) \in \mathbb{Q}[X]$.

c) The discrete analogue of partial integration follows from the product rule: For $f, g \in \mathcal{F}$ we have $\sigma(f \cdot \delta g)=\sigma \delta(f g)-\sigma(\delta f \cdot \tau g)=f g-(f g)(0)-\sigma(\delta f \cdot \tau g) \in \mathcal{F}$.

For example, we determine $\mathbb{N}_{0} \rightarrow \mathbb{N}_{0}: n \mapsto \sum_{i=0}^{n-1} i \cdot 2^{i}$ : Letting $f:=X \in \mathbb{Q}[X]$ and $g: \mathbb{N}_{0} \rightarrow \mathbb{N}_{0}: n \mapsto 2^{n}$, we get $\delta g=g$ and $\delta f=1 \in \mathbb{Q}[X]$, and thus $\sum_{i=0}^{n-1} i \cdot 2^{i}=(\sigma(f \cdot \delta g))(n)=(f g)(n)-(f g)(0)-(\sigma(\delta f \cdot \tau g))(n)=n \cdot 2^{n}-$ $\sum_{i=0}^{n-1} 2^{i+1}=n \cdot 2^{n}-\left(2^{n+1}-2\right)=(n-2) \cdot 2^{n}+2$.

For example, we determine $\mathbb{N}_{0} \rightarrow \mathbb{N}_{0}: n \mapsto \sum_{i=0}^{n-1} h_{i}$ : Letting $f: \mathbb{N}_{0} \rightarrow \mathbb{N}_{0}: n \mapsto$ $h_{n}$ and $g:=X \in \mathbb{Q}[X]$, we get $\delta f=\frac{1}{X+1} \in \mathbb{Q}(X)^{\circ}$ and $\delta g=1 \in \mathbb{Q}[X]$, and thus $\sum_{i=0}^{n-1} h_{i}=(\sigma(f \cdot \delta g))(n)=(f g)(n)-(f g)(0)-(\sigma(\delta f \cdot \tau g))(n)=$ $n h_{n}-\sum_{i=0}^{n-1} \frac{i+1}{i+1}=n\left(h_{n}-1\right)$.

(4.4) Example: Growth of partition numbers. The growth behavior of $p_{n, k}$, where $k \in \mathbb{N}$ is fixed while $n \in \mathbb{N}_{0}$ varies, is described by a quasipolynomial as follows: For $k \in \mathbb{N}$ and $a \in\{0, \ldots, k !-1\}$ there are $p_{a, k}(X) \in$ $\mathbb{Q}[X]$ such that $p_{n, k}=p_{a, k}(n)$, for all $n \in \mathbb{N}_{0}$ such that $n \equiv a(\bmod k !)$ and $[n, k] \neq[0,1]$. Moreover, $p_{a, k}(X)$ has degree $k-1$ and leading coefficient $\frac{1}{k !(k-1) !}$ for example, we have $p_{0,1}(X)=1$, and $p_{a, 2}(X)=\frac{X-a}{2}$ for $a \in\{0,1\}$ :

We proceed by induction on $k \in \mathbb{N}$ : We have $p_{n, 1}=1$ for $n \geq 1$. Moreover, since $p_{0,0}=1$ we have $p_{n, 0}+p_{n, 1}=1$ for all $n \in \mathbb{N}_{0}$, hence for completeness let additionally $p_{0,0}:=0 \in \mathbb{Q}[X]$. Note that $p_{n, 2}=p_{n-2,2}+p_{n-2,1}+p_{n-2,0}=$ $p_{n-2,2}+1$ yields $p_{n, 2}=\frac{n}{2}$ if $n \in \mathbb{N}_{0}$ is even, and $p_{n, 2}=\frac{n-1}{2}$ if $n \in \mathbb{N}$ is odd.

Let $k \geq 2$. For $n \geq k$ the recursion $p_{n, k}=\sum_{i=0}^{k} p_{n-k, i}$ yields $p_{n, k}-p_{n-k, k}=$ $\sum_{i=0}^{k-1} p_{(a-k)}(\bmod i !), i(n-k)$, which entails $p_{n, k}-p_{n-k !, k}=\sum_{j=0}^{(k-1) !-1}\left(p_{n-j k, k}-\right.$ $\left.p_{n-(j+1) k, k}\right)=\sum_{i=0}^{k-1} \sum_{j=1}^{(k-1) !} p_{(a-j k)}(\bmod i !), i(n-j k)$, for $n \geq k$ !. We apply discrete differentiation to the map $f_{a, k}: \mathbb{N}_{0} \rightarrow \mathbb{N}_{0}: m \mapsto p_{a+m k !, k}$ :

We have $\left(\delta f_{a, k}\right)(m)=\sum_{i=0}^{k-1} \sum_{j=1}^{(k-1) !} p_{(a-j k)}(\bmod i !), i((m+1) \cdot k !+a-j k)$, hence by induction $\delta f_{a, k}$ is a polynomial map in $m \in \mathbb{N}_{0}$ of degree $k-2$ with leading coefficient $(k-1) ! \cdot \frac{(k !)^{k-2}}{(k-1) !(k-2) !}=\frac{(k !)^{k-2}}{(k-2) !}$. Thus we infer that $f_{a, k}$ is a polynomial 
map in $m \in \mathbb{N}_{0}$ of degree $k-1$ with leading coefficient $\frac{(k !)^{k-2}}{(k-1) \cdot(k-2) !}=\frac{(k !)^{k-2}}{(k-1) !}$. Letting $n:=a+m k$ ! shows that $p_{n, k}$ is a polynomial map of degree $k-1$ with leading coefficient $\frac{1}{(k !)^{k-1}} \cdot \frac{(k !)^{k-2}}{(k-1) !}=\frac{1}{k !(k-1) !}$.

(4.5) Linear inversion. a) A sequence $\left[f_{0}, f_{1}, \ldots\right] \subseteq \mathbb{Q}[X]$ such that $\operatorname{deg}\left(f_{i}\right)=$ $i$ for all $i \in \mathbb{N}_{0}$, is called basic. Then, for all $n \in \mathbb{N}_{0}$, the truncated sequence $\left[f_{0}, \ldots, f_{n}\right] \subseteq \mathbb{Q}[X]$ is a $\mathbb{Q}$-basis of $\mathbb{Q}[X]_{\leq n}$.

Thus, if $\left[f_{0}, f_{1}, \ldots\right] \subseteq \mathbb{Q}[X]$ and $\left[g_{0}, g_{1}, \ldots\right] \subseteq \mathbb{Q}[X]$ are basic sequences, then there are lower triangular matrices $A=\left[a_{i j}\right]_{i j} \in \mathbb{Q}^{\mathbb{N}_{0} \times \mathbb{N}_{0}}$ and $B=\left[b_{i j}\right]_{i j} \in$ $\mathbb{Q}^{\mathbb{N}_{0} \times \mathbb{N}_{0}}$ such that $g_{i}=\sum_{j=0}^{i} a_{i j} f_{j} \in \mathbb{Q}[X]_{\leq i}$ and $f_{i}=\sum_{j=0}^{i} b_{i j} g_{j} \in \mathbb{Q}[X]_{\leq i}$, for all $i \in \mathbb{N}_{0}$. Thus we have $\sum_{k=j}^{i} a_{i k} b_{k j}=\delta_{i j} \in \mathbb{Q}$ and $\sum_{k=j}^{i} b_{i k} a_{k j}=\delta_{i j} \in \mathbb{Q}$, for all $i, j \in\{0, \ldots, n\}$. Note that the matrices $A_{n}:=\left[a_{i j}\right]_{i \in\{0, \ldots, n\}, j \in\{0, \ldots, n\}} \in$ $\mathbb{Q}^{(n+1) \times(n+1)}$ and $B_{n}:=\left[b_{i j}\right]_{i \in\{0, \ldots, n\}, j \in\{0, \ldots, n\}} \in \mathbb{Q}^{(n+1) \times(n+1)}$ are the associated base change matrices on $\mathbb{Q}[X]_{\leq n}$, which hence are inverse to each other.

Hence, if $\left[x_{0}, x_{1}, \ldots\right] \subseteq \mathbb{Q}$ is any sequence, and the sequence $\left[y_{0}, y_{1}, \ldots\right] \subseteq \mathbb{Q}$ is given by weighted sums as $y_{i}:=\sum_{j=0}^{i} a_{i j} x_{j} \in \mathbb{Q}$, for all $i \in \mathbb{N}_{0}$, then we may recover the original sequence by $x_{i}=\sum_{j=0}^{i} b_{i j} y_{j} \in \mathbb{Q}$, for all $i \in \mathbb{N}_{0}$ : For all $n \in \mathbb{N}_{0}$ we have $\left[y_{0}, \ldots, y_{n}\right]^{\text {tr }}=A_{n} \cdot\left[x_{0}, \ldots, x_{n}\right]^{\text {tr }} \in \mathbb{Q}^{1 \times(n+1)}$, which is equivalent to $\left[x_{0}, \ldots, x_{n}\right]^{\operatorname{tr}}=B_{n} \cdot\left[y_{0}, \ldots, y_{n}\right]^{\operatorname{tr}} \in \mathbb{Q}^{1 \times(n+1)}$.

b) For the basic sequences $\left[X^{n} ; n \in \mathbb{N}_{0}\right] \subseteq \mathbb{Q}[X]$ and $\left[(X-1)^{n} ; n \in \mathbb{N}_{0}\right] \subseteq \mathbb{Q}[X]$ we get $X^{n}=(X-1+1)^{n}=\sum_{k=0}^{n}\left(\begin{array}{c}n \\ k\end{array}\right)(X-1)^{k} \in \mathbb{Q}[X]_{\leq n}$ and $(X-1)^{n}=$ $\sum_{k=0}^{n}(-1)^{n-k}\left(\begin{array}{l}n \\ k\end{array}\right) X^{k} \in \mathbb{Q}[X]_{\leq n}$, with base change matrices $\left[\left(\begin{array}{l}n \\ k\end{array}\right)\right]_{n, k} \in \mathbb{Z}^{\mathbb{N}_{0} \times \mathbb{N}_{0}}$ and $\left[(-1)^{n-k}\left(\begin{array}{c}n \\ k\end{array}\right)\right]_{n, k} \in \mathbb{Z}^{\mathbb{N}_{0} \times \mathbb{N}_{0}}$, respectively. More symmetrically, replacing the first basic sequence by $\left[(-X)^{n} ; n \in \mathbb{N}_{0}\right] \subseteq \mathbb{Q}[X]$ we get the involutory base change matrix $\left[(-1)^{n}\left(\begin{array}{l}n \\ k\end{array}\right)\right]_{n, k} \in \mathbb{Z}^{\mathbb{N}_{0} \times \mathbb{N}_{0}}$.

Thus, for sequences $\left[y_{0}, y_{1}, \ldots\right] \subseteq \mathbb{Q}$ and $\left[x_{0}, x_{1}, \ldots\right] \subseteq \mathbb{Q}$ binomial inversion says that we have $y_{n}=\sum_{k=0}^{n}\left(\begin{array}{l}n \\ k\end{array}\right) x_{k} \in \mathbb{Q}$, for all $n \in \mathbb{N}_{0}$, if and only if we have $x_{n}=\sum_{k=0}^{n}(-1)^{n-k}\left(\begin{array}{l}n \\ k\end{array}\right) y_{k} \in \mathbb{Q}$, for all $n \in \mathbb{N}_{0}$. Moreover, if $y_{n}=\sum_{k=0}^{n}(-1)^{n}\left(\begin{array}{c}n \\ k\end{array}\right) x_{k} \in \mathbb{Q}$, for all $n \in \mathbb{N}_{0}$, then binomial inversion becomes involutory, that is $x_{n}=\sum_{k=0}^{n}(-1)^{n}\left(\begin{array}{l}n \\ k\end{array}\right) y_{k} \in \mathbb{Q}$, for all $n \in \mathbb{N}_{0}$.

In particular, considering the unit sequence $\left[x_{0}, x_{1}, \ldots\right]:=[0, \ldots, 0,1,0, \ldots] \subseteq$ $\mathbb{Z}$, where the non-vanishing entry is in position $m \in \mathbb{N}_{0}$, we get $y_{n}=\left(\begin{array}{l}n \\ m\end{array}\right) \in \mathbb{Z}$, for all $n \in \mathbb{N}_{0}$, and thus $\sum_{k=m}^{n}(-1)^{n-k}\left(\begin{array}{l}n \\ k\end{array}\right)\left(\begin{array}{l}k \\ m\end{array}\right)=\delta_{n, m} \in \mathbb{Z}$; note that for $m=0$ we recover the identity $\sum_{k=0}^{n}(-1)^{n-k}\left(\begin{array}{l}n \\ k\end{array}\right)=\delta_{0, n}$. Moreover, Newton expansion just says that any sequence $f:=\left[f_{i} ; i \in \mathbb{N}_{0}\right] \subseteq \mathbb{Q}$ is related to the sequence $\left[\left(\delta^{i} f\right)(0) ; i \in \mathbb{N}_{0}\right] \subseteq \mathbb{Q}$ by binomial inversion.

For example, we reconsider the number $D_{n}$ of derangements in $\mathcal{S}_{n}$, for $n \in \mathbb{N}_{0}$ : Let $D_{n, k} \in \mathbb{N}_{0}$ be the number of permutations in $\mathcal{S}_{n}$ having precisely $k$ fixed points, for $k \in \mathbb{N}_{0}$; hence we have $D_{n}=D_{n, 0}$. Choosing any $k$-subset of $\{1, \ldots, n\}$ as set of fixed points, we get $n !=\sum_{k=0}^{n}\left(\begin{array}{l}n \\ k\end{array}\right) D_{n-k}=\sum_{k=0}^{n}\left(\begin{array}{l}n \\ k\end{array}\right) D_{k}$. 
Thus binomial inversion yields $D_{n}=\sum_{k=0}^{n}(-1)^{n-k}\left(\begin{array}{l}n \\ k\end{array}\right) \cdot k !=n ! \cdot \sum_{k=0}^{n} \frac{(-1)^{n-k}}{(n-k) !}=$ $n ! \cdot \sum_{k=0}^{n} \frac{(-1)^{k}}{k !}$, and Newton expansion becomes $D_{n}=\left(\delta^{n}(k \mapsto k !)\right)(0)$.

c) For the basic sequences $\left[X^{n} ; n \in \mathbb{N}_{0}\right] \subseteq \mathbb{Q}[X]$ and $\left[X_{(n)} ; n \in \mathbb{N}_{0}\right] \subseteq \mathbb{Q}[X]$ we have $X^{n}=\sum_{k=0}^{n} S_{n, k} X_{(k)} \in \mathbb{Q}[X]_{\leq n}$ and $X_{(n)}=\sum_{k=0}^{n}(-1)^{n-k} s_{n, k} X^{k} \in$ $\mathbb{Q}[X]_{\leq n}$, with base change matrices $\left[S_{n, k}\right]_{n, k} \in \mathbb{Z}^{\mathbb{N}_{0} \times \mathbb{N}_{0}}$ and $\left[(-1)^{n-k} s_{n, k}\right]_{n, k} \in$ $\mathbb{Z}^{\mathbb{N}_{0} \times \mathbb{N}_{0}}$, respectively. Again, replacing replacing the first basic sequence by $\left[(-X)^{n} ; n \in \mathbb{N}_{0}\right] \subseteq \mathbb{Q}[X]$ we get the base change matrices $\left[(-1)^{n} S_{n, k}\right]_{n, k} \in$ $\mathbb{Z}^{\mathbb{N}_{0} \times \mathbb{N}_{0}}$ and $\left[(-1)^{n} s_{n, k}\right]_{n, k} \in \mathbb{Z}^{\mathbb{N}_{0} \times \mathbb{N}_{0}}$, respectively.

Thus, for sequences $\left[y_{0}, y_{1}, \ldots\right] \subseteq \mathbb{Q}$ and $\left[x_{0}, x_{1}, \ldots\right] \subseteq \mathbb{Q}$ Stirling inversion says that we have $y_{n}=\sum_{k=0}^{n} S_{n, k} x_{k} \in \mathbb{Q}$, for all $n \in \mathbb{N}_{0}$, if and only if we have $x_{n}=\sum_{k=0}^{n}(-1)^{n-k} s_{n, k} y_{k} \in \mathbb{Q}$, for all $n \in \mathbb{N}_{0}$. Moreover, if $y_{n}=\sum_{k=0}^{n}(-1)^{n} S_{n, k} x_{k} \in \mathbb{Q}$, for all $n \in \mathbb{N}_{0}$, then Stirling inversion becomes more symmetric inasmuch $x_{n}=\sum_{k=0}^{n}(-1)^{n} s_{n, k} y_{k} \in \mathbb{Q}$, for all $n \in \mathbb{N}_{0}$. In particular, considering the $m$-th unit sequence again, where $m \in \mathbb{N}_{0}$, we for all $n \in \mathbb{N}_{0}$ recover $\sum_{k=m}^{n}(-1)^{n-k} s_{n, k} S_{k, m}=\delta_{n, m} \in \mathbb{Z}$.

\section{Posets}

\section{$5 \quad$ Partially ordered sets}

(5.1) Partially ordered sets. a) A set $X \neq \emptyset$, together with a binary relation $\leq$ on $X$, is called a partially ordered set or poset if the following properties are fulfilled for all $x, y, z \in X$ : We have reflexivity, that is $x \leq x$; antisymmetry, that is $x \leq y$ and $y \leq x$ implies $x=y$; and transitivity, that is $x \leq y$ and $y \leq z$ implies $x \leq z$. Elements $x, y \in X$ are called comparable if $x \leq y$ or $y \leq x$; and we write $x<y$ whenever $x \leq y$ and $x \neq y$. Note that we get the dual partially ordered set by letting $x \leq^{\prime} y$ if and only if $y \leq x$, for all $x, y \in X$.

Partially ordered sets $X$ and $Y$, with comparison relations $\leq_{X}$ and $\leq_{Y}$, are called isomorphic, if there is a bijection $\alpha: X \rightarrow Y$ such that $x \leq_{X} y$ if and only if $\alpha(x) \leq_{Y} \alpha(y)$, for all $x, y \in X$; we write $X \cong Y$. In particular, any subset $\emptyset \neq Y \subseteq X$ becomes a partially ordered set again, with respect to the induced comparison relation, which is defined by saying that the natural embedding $\iota: Y \rightarrow X$ yields an isomorphism $Y \cong \operatorname{im}(\iota)$.

A subset $Y \subseteq X$, such that whenever $y \in Y$ and $x \in X$ such that $x \leq y$ we already have $x \in Y$, is called an ideal of $X$. Similarly, a subset $Y \subseteq X$, such that whenever $y \in Y$ and $x \in X$ such that $x \geq y$ we already have $x \in Y$, is called a coideal of $X$. In particular, for any $x \in X$ the sets $\langle\leq x\rangle:=\{y \in X ; y \leq x\}$ and $\langle x \leq\rangle:=\{y \in X ; x \leq y\}$ are called the principal ideal and coideal generated by $x$, respectively.

b) An element $x \in X$ is called minimal, if $y \leq x \in X$ already implies $y=x$; dually, $x \in X$ is called maximal if $x \leq y \in X$ already implies $y=x$. In 
particular, if $X$ is finite then for any $x \in X$ there is a minimal element $y \in X$ such that $y \leq x$, and a maximal element $z \in X$ such that $x \leq z$. Moreover, an element $\underline{0} \in X$ such that $\underline{0} \leq x$ for all $x \in X$ is called a zero element; dually, an element $\underline{1} \in X$ such that $x \leq \underline{1}$ for all $x \in X$ is called a one element; note that zero and one elements are the unique minimal and maximal elements, respectively, if existent.

Given a subset $\emptyset \neq Y \subseteq X$, an element $x \in X$ such that $y \leq x$ for all $y \in Y$ is called an upper bound of $Y$. If $Y$ has an upper bound, and the set of upper bounds has a zero element $x$, with respect to the induced comparison relation, then $x \in X$ is called a least upper bound or join or supremum of $Y$; if existent, the least upper bound is unique, and denoted by $\bigvee_{y \in Y} y$. Dually, an element $x \in X$ such that $x \leq y$ for all $y \in Y$ is called a lower bound of $Y$. If $Y$ has a lower bound, and the set of lower bounds has a one element $x$, with respect to the induced comparison relation, then $x \in X$ is called a greatest lower bound bound or meet or infimum of $Y$; if existent, the greatest lower bound is unique, and denoted by $\bigwedge_{y \in Y} y$.

c) The partially ordered set $X$ is called a lattice, if for all $x, y \in X$ there is a join $x \vee y \in X$ and a meet $x \wedge y \in X$. In this case, for all $x, y, z \in X$ we have idempotency $x \wedge x=x$ and commutativity $x \wedge y=y \wedge x$, and dually $x \vee x=x$ and $x \vee y=y \vee x$; moreover, $(x \wedge y) \wedge z \in X$ is the greatest lower bound of $\{x, y, z\} \in X$, hence we infer associativity $(x \wedge y) \wedge z=x \wedge(y \wedge z)$, and dually $(x \vee y) \vee z=x \vee(y \vee z)$; finally $x \wedge y=x$ is equivalent to $x \leq y$, which in turn is equivalent to $x \vee y=y$.

In particular, if $X$ is finite, then $\underline{0}:=\bigwedge_{x \in X} x \in X$ and $\underline{1}:=\bigvee_{x \in X} x \in X$ are a zero and a one element, respectively.

(5.2) Chains. a) Let $X$ be a partially ordered set. Given $x \leq y \in X$, the associated (closed) interval is the set $[x, y]:=\{z \in X ; x \leq z \leq y\}$; in particular, we have $[x, x]=\{x\}$. The partially ordered set $X$ is called locally finite if all its intervals are finite sets.

Given $x<y$, we say that $y$ covers $x$ if $[x, y]=\{x, y\}$, and we write $x \lessdot y$. In particular, if $\underline{0} \in X$ is a zero element, then an element $x \in X$ such that $\underline{0} \lessdot x$ is called an atom; similarly, if $\underline{1} \in X$ is a one element, then an element $x \in X$ such that $x \lessdot \underline{1}$ is called a co-atom. If $X$ is finite, then it is typically depicted by its Hasse diagram, which is a quiver, that is a simple oriented graph, with vertex set $X$ and arrows $x \leftarrow y$ whenever $x \lessdot y$.

A subset $\emptyset \neq Y \subseteq X$ is called a chain or totally ordered if all $x, y \in Y$ are pairwise comparable; in particular any chain is a lattice. Note that Zorn's Lemma (being equivalent to the Axiom of Choice) says that, if any chain $Y \subseteq X$ has an upper bound, then $X$ has a maximal element.

A chain $Y \subseteq X$ is called maximal if for any chain $Y^{\prime} \subseteq X$ such that $Y \subseteq Y^{\prime}$ we already have $Y=Y^{\prime}$. A chain $Y \subseteq X$ is called saturated or unrefinable if for all $x \leq y \in Y$ the chain $Y \cap[x, y] \subseteq[x, y]$ is maximal. Hence maximal chains 
are saturated, while the converse does not hold; note that a saturated chain is maximal if it contains both a minimal and a maximal element of $X$.

The set of all chains in $X$ is partially ordered by set-theoretic inclusion, and any chain of chains has its union, which again is a chain, as an upper bound, hence by Zorn's Lemma any chain is contained in a maximal chain. Of course, if $X$ is finite, then any chain can be refined to a maximal chain by induction.

For example, the set $\mathbb{N}_{0}$ is a partially ordered set with respect to the $\leq$ relation. It is a locally finite chain, thus in particular a lattice, with meet $\min \{i, j\}$ and join $\max \{i, j\}$, for $i, j \in \mathbb{N}_{0}$; it has zero element 0 , but no maximal element. The subset $[0, n]:=\{0, \ldots, n\}$, where $n \in \mathbb{N}_{0}$, is totally ordered of length $n$, with zero element 0 and one element $n$. Note that for any interval $[m, n]$ in $\mathbb{N}_{0}$, where $m \leq n$, we have $[m, n] \cong[0, m-n]$ as partially ordered sets; moreover, any finite totally ordered set $X$ is isomorphic to $[0,|X|-1]$.

b) Let $Y=\left\{x_{0}, \ldots, x_{n}\right\} \subseteq X$ be a finite chain of length $l(Y):=n \in \mathbb{N}_{0}$, where we may assume that $x_{0}<x_{1}<\cdots<x_{n}$; in particular, $x_{0}$ and $x_{n}$ are its zero and one elements, respectively, and $Y$ is saturated if and only if $x_{i-1} \lessdot x_{i}$, for all $i \in\{1, \ldots, n\}$. The length of a partially ordered set $X$ is defined as $l(X):=$ $\max \left\{l(Y) \in \mathbb{N}_{0} ; Y \subseteq X\right.$ finite chain $\} \in \mathbb{N}_{0} \dot{\cup}\{\infty\}$. If $X$ is finite, then we have $l(X) \in \mathbb{N}_{0}$, where it of course suffices to consider the maximal chains only; if $X$ is locally finite, then for $x \leq y$ we write $l(x, y):=l([x, y]) \in \mathbb{N}_{0}$. Moreover, $X$ is said to be graded of length $n \in \mathbb{N}_{0}$, if all its maximal chains have finite length $n$; then for $x \leq y$ the interval $[x, y]$ is graded of length $l(x, y) \leq n$.

If $X$ is graded of length $n \in \mathbb{N}_{0}$, then there is a well-defined length map $l: X \rightarrow\{0, \ldots, n\}$, for $x \in X$ given by $l(x):=l\left(x^{\prime}, x\right)$, where $x^{\prime} \in X$ is any minimal element such that $x^{\prime} \leq x$. Thus, in particular, if $x \in X$ is a minimal element then we have $l(x)=0$, for $x \leq y$ we have $l(y)-l(x)=l(x, y)$, and for $x \lessdot y$ we have $l(y)=l(x)+1$. This is seen as follows:

Note first, since any maximal chain has finite length, there is a minimal element $x^{\prime} \in X$ as desired, and similarly there is a maximal element $y \in X$ such that $x \leq y$. Now, if $x^{\prime \prime} \in X$ also is a minimal element such that $x^{\prime \prime} \leq x$, then letting $X^{\prime} \subseteq\left[x^{\prime}, x\right]$ and $X^{\prime \prime} \subseteq\left[x^{\prime \prime}, x\right]$ be maximal chains, and choosing a maximal chain $Y \subseteq[x, y]$, yields maximal chains $X^{\prime} \cup Y$ and $X^{\prime \prime} \cup Y$ in $X$, hence $l\left(x^{\prime}, x\right)=$ $l\left(X^{\prime}\right)=l\left(X^{\prime} \cup Y\right)-l(Y)=l\left(X^{\prime \prime} \cup Y\right)-l(Y)=l\left(X^{\prime \prime}\right)=l\left(x^{\prime \prime}, x\right)$.

For example, let $X:=\{\emptyset,\{1\},\{2\},\{2,3\},\{1,2,3\}\}$, partially ordered by settheoretic inclusion $\subseteq$. By inspection, $X$ is a lattice, having $\emptyset$ and $\{1,2,3\}$ as its zero and one elements, respectively. But while meets are given by settheoretic intersections, this is not the case for joins: we have $\{1\} \vee\{2\}=\{1,2,3\}$. Moreover, both $\emptyset \subset\{1\} \subset\{1,2,3\}$ and $\emptyset \subset\{2\} \subset\{2,3\} \subset\{1,2,3\}$ are maximal chains, of lengths 2 and 3 , respectively, thus $X$ is not graded.

(5.3) Example: Subset lattices. Let $N$ be a set. Then the finitary power set $\mathcal{P}_{\text {fin }}(N) \subseteq \mathcal{P}(N)$ consisting of the finite subsets of $N$, and the co-finitary power set $\mathcal{P}_{\text {co-fin }}(N) \subseteq \mathcal{P}(N)$ consisting of the subsets of $N$ having finite com- 
plements, are partially ordered by set-theoretic inclusion $\subseteq$. They are locally finite lattices with meet $M \cap M^{\prime}$ and join $M \cup M^{\prime}$. Moreover, $\mathcal{P}_{\text {fin }}(N)$ has $\emptyset$ as its zero element, and it has a one element if and only if $N$ is finite, in this case coinciding with $N$; and $\mathcal{P}_{\text {co-fin }}(N)$ has $N$ as one element, and it has a zero element if and only if $N$ is finite, in this case coinciding with $\emptyset$.

If $N$ is finite of cardinality $n \in \mathbb{N}_{0}$, that is we have $\mathcal{P}(N)=\mathcal{P}_{\text {fin }}(N)=\mathcal{P}_{\text {co-fin }}(N)$, all maximal chains are of the form $\emptyset=M_{0} \subset M_{1} \subset \cdots \subset M_{n}=N$, where $\left|M_{i}\right|=i$ for all $i \in\{0, \ldots, n\}$. Hence $\mathcal{P}(N)$ is graded of length $n$, where $M \subseteq N$ has length $|M|$. Then $\mathcal{P}(N)$ has $\left(\begin{array}{l}n \\ k\end{array}\right)$ elements of length $k \in\{0, \ldots, n\}$, that is $k$-subsets, and is totally ordered if and only if $n \leq 1$. For example, the Hasse diagram of $\mathcal{P}(\{1,2,3\})$ is depicted on the left hand side of Table 9 , where the vertices are labeled as follows: The subsets of $\{1,2,3\}$ are identified with the indicator functions $\operatorname{Maps}(\{1,2,3\},\{0,1\})$, and the latter in turn are identified with the 2 -adic representations of the numbers $\{0, \ldots, 7\}$.

(5.4) Example: Subspace lattices. Let $\mathbb{F}_{q}$ be the finite field of order $q$, and for $n \in \mathbb{N}_{0}$ let $\mathbf{P}_{n}(q)$ be the set of $\mathbb{F}_{q}$-subspaces of $\mathbb{F}_{q}^{n}$, partially ordered by settheoretic inclusion $\subseteq$. Then $\mathbf{P}_{n}(q)$ is a lattice with meet $V \cap V^{\prime}$ and join $V+V^{\prime}$, for $V, V^{\prime} \leq \mathbb{F}_{q}^{n}$, and has $\{0\}$ and $\mathbb{F}_{q}^{n}$ as its zero and one elements, respectively. Note that $\mathbf{P}_{n}(q)$ is totally ordered if and only if $n \leq 1$. All maximal chains are of the form $\emptyset=V_{0}<V_{1}<\cdots<V_{n}=\mathbb{F}_{q}^{n}$, where $\operatorname{dim}_{\mathbb{F}_{q}}\left(V_{i}\right)=i$ for all $i \in\{0, \ldots, n\}$. Hence $\mathbf{P}_{n}(q)$ is graded of length $n$, where $V \leq \mathbb{F}_{q}^{n}$ has length $\operatorname{dim}_{\mathbb{F}_{q}}(V)$. Counting sequences of $\mathbb{F}_{q}$-linearly independent sequences we conclude that $\mathbf{P}_{n}(q)$ has $\left(\begin{array}{l}n \\ k\end{array}\right)_{q}:=\prod_{i=0}^{k-1} \frac{q^{n}-q^{i}}{q^{k}-q^{i}}=\prod_{i=0}^{k-1} \frac{q^{n-i}-1}{q^{k-i}-1}=\prod_{i=0}^{k-1} \frac{q^{n-i}-1}{q^{i+1}-1}$ $\mathbb{F}_{q}$-subspaces of $\mathbb{F}_{q}$-dimension $k \in\{0, \ldots, n\}$, that is of length $k$.

Counting differently, using a fixed embedding $\mathbb{F}_{q}^{n-1} \leq \mathbb{F}_{q}^{n}$, for $n \geq 1$, for any $k$ dimensional $\mathbb{F}_{q}$-subspace $V \leq \mathbb{F}_{q}^{n}$, for $k \in\{1, \ldots, n\}$, we have either $V \leq \mathbb{F}_{q}^{n-1}$ or

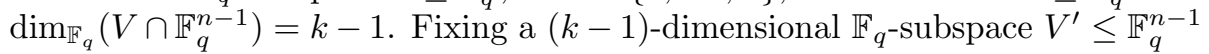
and going over to $\mathbb{F}_{q}^{n} / V^{\prime}$, shows that there are $\frac{q^{n-k+1}-1}{q-1}-\frac{q^{n-k}-1}{q-1}=q^{n-k}$ subspaces $V$ as above such that $V \cap \mathbb{F}_{q}^{n-1}=V^{\prime}$. Thus we get the triangle identity $\left(\begin{array}{l}n \\ k\end{array}\right)_{q}=\left(\begin{array}{c}n-1 \\ k\end{array}\right)_{q}+q^{n-k} \cdot\left(\begin{array}{l}n-1 \\ k-1\end{array}\right)_{q}$, for all $n \in \mathbb{N}$ and $k \in\{1, \ldots, n\}$. Since $\left(\begin{array}{l}n \\ 0\end{array}\right)_{q}=1$, for all $n \in \mathbb{N}_{0}$, we see that $\left(\begin{array}{l}n \\ k\end{array}\right)_{q}$ can be considered as an element of the polynomial $\operatorname{ring} \mathbb{Z}[q]$ in the indeterminate $q$, called a Gaussian polynomial.

Since for $i \in \mathbb{N}$ we have $q^{i}-1=(q-1) \cdot \sum_{j=0}^{i-1} q^{j} \in \mathbb{Z}[q]$, we may specialize $\left(\begin{array}{l}n \\ k\end{array}\right)_{q}=\prod_{i=0}^{k-1} \frac{q^{n-i}-1}{q^{i+1}-1}=\prod_{i=0}^{k-1} \frac{\sum_{j=0}^{n-i-1} q^{j}}{\sum_{j=0}^{i} q^{j}} \in \mathbb{Q}(q)$ at $q \mapsto 1$, yielding $\left.\left(\begin{array}{l}n \\ k\end{array}\right)_{q}\right|_{q \mapsto 1}=$ $\prod_{i=0}^{k-1} \frac{n-i}{i+1}=\left(\begin{array}{l}n \\ k\end{array}\right)$. Thus $\left(\begin{array}{l}n \\ k\end{array}\right)_{q}$ is called the $q$-analogue of the binomial coefficient $\left(\begin{array}{l}n \\ k\end{array}\right)$; since the latter coincides with the number of elements of length $k$ in the partially ordered set $\mathcal{P}(N)$ where $N$ has cardinality $n$, in this sense $\mathcal{P}(N)$ is called 'the $n$-dimensional vector space over the field with one element'.

For example, $\mathbf{P}_{2}(2)$ is isomorphic to $\{\emptyset,\{1\},\{2\},\{3\},\{1,2,3\}\}$, partially ordered 
Table 9: The cube and the Fano plane.
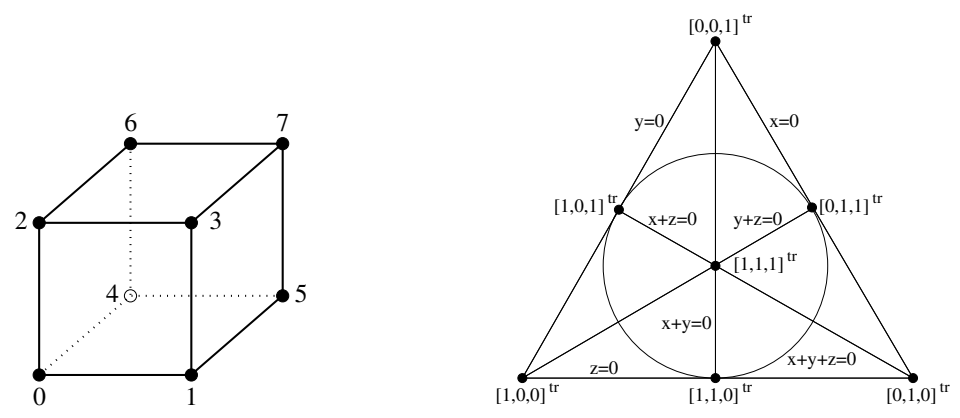

by set-theoretic inclusion $\subseteq$, and $\mathbf{P}_{3}(2)$ is isomorphic to

$$
\begin{aligned}
\{\emptyset, & \{1\},\{2\},\{3\},\{4\},\{5\},\{6\},\{7\}, \quad\{1, \ldots, 7\}, \\
& \{1,2,3\},\{1,4,5\},\{2,4,6\},\{1,6,7\},\{2,5,7\},\{3,4,7\},\{3,5,6\}\} .
\end{aligned}
$$

The subset $\mathbf{P}_{3}(2) \backslash\left\{\{0\}, \mathbb{F}_{2}^{3}\right\}$ is called the Fano plane or the projective plane of order 2 ; it is depicted in Table 9 , where 1 -dimensional $\mathbb{F}_{2}$-subspaces are given as vertices, labeled by generating vectors, and 2-dimensional $\mathbb{F}_{2}$-subspaces are given as lines, labeled by defining linear equations, and where the numbers $\{1, \ldots, 7\}$ are identified with vectors by going over to 2 -adic representations.

(5.5) Example: Divisibility lattices. We consider the basic example from number theory: the set $\mathbb{N}$, partially ordered by divisibility $\mid$. Then $\mathbb{N}$ is a locally finite lattice, with meet $\operatorname{gcd}(c, d)$ and join $\operatorname{lcm}(c, d)$, for $c, d \in \mathbb{N}$; it has 1 as its zero element, but does not have a one element. Indeed, for $n \in \mathbb{N}$, the interval in $\mathbb{N}$ between 1 and $n$ is given as $X_{n}:=\{d \in \mathbb{N} ; d \mid n\}$, that is the set of divisors of $n \in \mathbb{N}$, and the interval between $d \mid n \in \mathbb{N}$ is isomorphic to $X_{\frac{n}{d}}$.

All maximal chains in $X_{n}$ are of the form $1=d_{0}\left|d_{1}\right| \cdots \mid d_{k}=n$, where $\frac{d_{i}}{d_{i-1}} \in \mathbb{N}$ is a prime, for all $i \in\{1, \ldots, k\}$, and where $k \in \mathbb{N}_{0}$ is the length of the prime factorization of $n$, counting multiplicities. Hence $X_{n}$ is graded of length $k$, where the length of $d \in X_{n}$ is given by the length of its prime factorization; note that $X_{n}$ is totally ordered if and only if $n$ is a prime power.

(5.6) Example: Dominance partial order. a) We consider the set $P(n)$ of partitions of $n \in \mathbb{N}_{0}$, where for $\lambda:=\left[\lambda_{1}, \ldots, \lambda_{k}\right] \vdash n$ with $k \in\{0, \ldots, n\}$ parts we let $\lambda_{i}:=0$ for all $i>k$, thus we may write $\lambda=\left[\lambda_{1}, \ldots, \lambda_{n}\right]$. Then $P(n)$ is partially ordered by dominance $\unlhd$, where $\lambda=\left[\lambda_{1}, \ldots, \lambda_{n}\right] \vdash n$ is said to dominate $\mu=\left[\mu_{1}, \ldots, \mu_{n}\right] \vdash n$ if $\sum_{i=1}^{k} \mu_{i} \leq \sum_{i=1}^{k} \lambda_{i}$, for all $k \in\{1, \ldots, n\}$ : 
It is immediate that reflexivity and transitivity hold. Moreover, from $\mu \leq \lambda$ and $\lambda \leq \mu$ we get $\sum_{i=1}^{k} \mu_{i}=\sum_{i=1}^{k} \lambda_{i}$, for all $k \in\{1, \ldots, n\}$, which successively entails $\mu_{i}=\lambda_{i}$, for all $k \in\{1, \ldots, n\}$. Hence we have antisymmetry as well, showing that dominance $\unlhd$ indeed is a partial order. Actually, $P(n)$ is a lattice with respect to the partial order $\unlhd$.

We describe the associated covering relation: Given $\mu \vdash n$, we have $\mu \lessdot \lambda$ if and only if $\lambda=\left[\mu_{1}, \ldots, \mu_{r-1}, \mu_{r}+1, \mu_{r+1}, \ldots, \mu_{s-1}, \mu_{s}-1, \mu_{s+1}, \ldots, \mu_{n}\right]$, where $1 \leq r<s \leq n$ such that $\mu_{s}>\mu_{s+1}$ and $\mu_{r-1}>\mu_{r}$, if $r>1$, and such that either $s=r+1$, or $s>r+1$ and $\mu_{r}=\mu_{s}$ :

If $\mu \lessdot \lambda$, then let $r:=\min \left\{i \in\{1, \ldots, n\} ; \mu_{i} \neq \lambda_{i}\right\}$ and $s:=\min \{k \in\{r+$ $\left.1, \ldots, n\} ; \sum_{i=1}^{k} \mu_{i}=\sum_{i=1}^{k} \lambda_{i}\right\}$, thus $1 \leq r<s \leq n$. Hence we have $\mu_{r}<\lambda_{r}$, and $\lambda_{r} \leq \lambda_{r-1}=\mu_{r-1}$ if $r>1$, as well as $\mu_{s}>\lambda_{s} \geq \lambda_{s+1} \geq \mu_{s+1}$. This yields $\mu \triangleleft \nu:=\left[\mu_{1}, \ldots, \mu_{r-1}, \mu_{r}+1, \mu_{r+1}, \ldots, \mu_{s-1}, \mu_{s}-1, \mu_{s+1}, \ldots, \mu_{n}\right] \unlhd \lambda$, hence $\nu=\lambda$. It remains to show $\mu_{r}=\mu_{s}$ whenever $s>r+1$ : Assume to the contrary that $\mu_{r}>\mu_{s}$, and let $r<t:=\min \left\{i \in\{r+1, \ldots, s\} ; \mu_{i-1}>\mu_{i}\right\} \leq s$. If $t=s$ then $\mu \triangleleft\left[\mu_{1}, \ldots, \mu_{r-1}, \mu_{r}+1, \mu_{r+1}, \ldots, \mu_{s-2}, \mu_{s-1}-1, \mu_{s}, \ldots, \mu_{n}\right] \triangleleft \nu=\lambda$, while if $t<s$ then $\mu \triangleleft\left[\mu_{1}, \ldots, \mu_{r}, \ldots, \mu_{t-1}, \mu_{t}+1, \mu_{t+1}, \ldots, \mu_{s-1}, \mu_{s}-1, \mu_{s+1}, \ldots, \mu_{n}\right] \triangleleft$ $\nu=\lambda$, a contradiction.

Let conversely $\lambda$ be as asserted, and let $\nu=\left[\nu_{1}, \ldots, \nu_{n}\right] \vdash n$ such that $\mu \triangleleft \nu \unlhd \lambda$. Hence for $i \notin\{r, \ldots, s\}$ we have $\nu_{i}=\mu_{i}$. Thus if $s=r+1$ we conclude $\nu_{r}=\mu_{r}+1$ and $\nu_{r+1}=\mu_{r+1}-1$, thus $\nu=\lambda$. If $s>r+1$ and hence $\mu_{r}=\mu_{s}$, then there are $r \leq r^{\prime}<s^{\prime} \leq s$ such that $\nu_{i}=\mu_{i}$ for $i \notin\left\{r^{\prime}, s^{\prime}\right\}$ as well as $\nu_{r^{\prime}}=\mu_{r^{\prime}}+1$ and $\nu_{s^{\prime}}=\mu_{s^{\prime}}-1$. Since $\mu_{r^{\prime}}=\nu_{r^{\prime}}-1 \leq \nu_{r^{\prime}-1}-1=\mu_{r^{\prime}-1}-1<\mu_{r^{\prime}-1}$, whenever $r^{\prime}>1$, and $\mu_{s^{\prime}}=\nu_{s^{\prime}}+1 \geq \nu_{s^{\prime}+1}+1=\mu_{s^{\prime}+1}+1>\mu_{s^{\prime}+1}$, this implies $r^{\prime}=r$ and $s^{\prime}=s$, hence $\nu=\lambda$ in this case as well.

For example, we have $\lambda \unlhd[n]$ and $\left[1^{n}\right] \unlhd \lambda$ for all $\lambda \vdash n$ and $n \in \mathbb{N}_{0}$, and $[n-1,1] \lessdot[n]$ for $n \geq 2$, and $\left[1^{3}\right] \lessdot[2,1] \lessdot[3]$ and $\left[1^{4}\right] \lessdot\left[2,1^{2}\right] \lessdot\left[2^{2}\right] \lessdot[3,1] \lessdot[4]$ and $\left[1^{4}\right] \lessdot\left[2,1^{3}\right] \lessdot\left[2^{2}, 1\right] \lessdot[3,2] \lessdot[4,1] \lessdot[5]$, and

$\left[1^{6}\right] \lessdot\left[2,1^{4}\right] \lessdot\left[2^{2}, 1^{2}\right] \lessdot\left\{\left[3,1^{3}\right],\left[2^{3}\right]\right\} \lessdot[3,2,1] \lessdot\left\{\left[4,1^{2}\right],\left[3^{2}\right]\right\} \lessdot[4,2] \lessdot[5,1] \lessdot[6]$,

where $\left\{\left[3,1^{3}\right],\left[2^{3}\right]\right\}$ and $\left\{\left[4,1^{2}\right],\left[3^{2}\right]\right\}$ are non-comparable.

b) Depicting $\lambda \vdash n$ by a Young diagram, the conjugate partition $\lambda^{\prime} \vdash n$ is obtained by reflecting the diagram along its main diagonal. Formally, if $\lambda=$ $\left[\lambda_{1}, \ldots, \lambda_{n}\right] \vdash n$ then letting $\lambda_{i}^{\prime}:=\left|\left\{j \in \mathbb{N} ; \lambda_{j} \geq i\right\}\right| \in \mathbb{N}_{0}$ for all $i \in \mathbb{N}$, we have $\lambda_{1}^{\prime} \geq \cdots \geq \lambda_{n}^{\prime} \geq 0$ and $\sum_{i=1}^{n} \lambda_{i}^{\prime}=\sum_{j=1}^{n}\left|\left\{i \in\{1, \ldots, n\} ; i \leq \lambda_{j}\right\}\right|=\sum_{j=1}^{n} \lambda_{j}=$ $n$, hence we indeed may let $\lambda^{\prime}:=\left[\lambda_{1}^{\prime}, \ldots, \lambda_{n}^{\prime}\right] \vdash n$. Moreover, conjugating twice yields $\lambda^{\prime \prime} \vdash n$, where $\lambda_{i}^{\prime \prime}=\left|\left\{j \in \mathbb{N} ; \lambda_{j}^{\prime} \geq i\right\}\right|=\left|\left\{j \in \mathbb{N} ;\left|\left\{k \in \mathbb{N} ; \lambda_{k} \geq j\right\}\right| \geq i\right\}\right|=$ $\mid\left\{j \in \mathbb{N} ;\{1, \ldots, i\} \subseteq\left\{k \in \mathbb{N} ; \lambda_{k} \geq j\right\}|=|\left\{j \in \mathbb{N} ; \lambda_{i} \geq j\right\}|=|\left\{1, \ldots, \lambda_{i}\right\} \mid=\lambda_{i}\right.$, that is we indeed have $\lambda^{\prime \prime}=\lambda$.

Alternatively, writing $\lambda^{\prime}=\left[n^{a_{n}^{\prime}}, \ldots, 1^{a_{1}^{\prime}}\right] \vdash n$ in terms of multiplicities, we have $a_{i}^{\prime}=\left|\left\{j \in \mathbb{N} ; \lambda_{j}^{\prime}=i\right\}\right|=\left|\left\{j \in \mathbb{N} ;\left|\left\{k \in \mathbb{N} ; \lambda_{k} \geq j\right\}\right|=i\right\}\right|=\mid\{j \in \mathbb{N} ;\{k \in$ $\left.\left.\mathbb{N} ; \lambda_{k} \geq j\right\}=\{1, \ldots, i\}\right\}|=|\left\{j \in \mathbb{N} ; \lambda_{i} \geq j, \lambda_{i+1}<j\right\}|=|\left\{\lambda_{i+1}+1, \ldots, \lambda_{i}\right\} \mid=$ $\lambda_{i}-\lambda_{i+1}$, for all $i \in\{1, \ldots, n\}$, providing the fastest way to compute conjugate 
partitions. For example, we have $[n]^{\prime}=\left[1^{n}\right]$ for all $n \in \mathbb{N}_{0}$, and $[n-1,1]^{\prime}=$ $\left[2,1^{n-2}\right]$ for $n \geq 2$, as well as $\left[2^{2}\right]^{\prime}=\left[2^{2}\right]$ and $[3,2]^{\prime}=\left[2^{2}, 1\right]$ and $\left[3,1^{2}\right]^{\prime}=\left[3,1^{2}\right]$.

Then we have $\mu \unlhd \lambda$ if and only if $\lambda^{\prime} \unlhd \mu^{\prime}$ : To show this, it suffices to assume to the contrary that $\mu \unlhd \lambda$ but $\lambda^{\prime} \unlhd \mu^{\prime}$. Then for some $k \in \mathbb{N}$ we have $\sum_{i=1}^{j} \lambda_{i}^{\prime} \leq$ $\sum_{i=1}^{j} \mu_{i}^{\prime}$ for all $j \in\{1, \ldots, k-1\}$, and $\sum_{i=1}^{k} \lambda_{i}^{\prime}>\sum_{i=1}^{k} \mu_{i}^{\prime}$. Hence we have $\lambda_{k}^{\prime}>\mu_{k}^{\prime}$ and $\sum_{i=k+1}^{n} \lambda_{i}^{\prime}<\sum_{i=k+1}^{n} \mu_{i}^{\prime}$. Now we have $\sum_{i=k+1}^{n} \lambda_{i}^{\prime}=\sum_{i=k+1}^{n} \mid\{j \in$ $\left.\mathbb{N} ; i \leq \lambda_{j}\right\} \mid=\sum_{j=1}^{\lambda_{k}^{\prime}}\left(\lambda_{j}-k\right)$ and similarly $\sum_{i=k+1}^{n} \mu_{i}^{\prime}=\sum_{j=1}^{\mu_{k}^{\prime}}\left(\mu_{j}-k\right)$; note that $\lambda_{j} \geq k$ for $j \in\left\{1, \ldots, \lambda_{k}^{\prime}\right\}$. This implies $\sum_{j=1}^{\mu_{k}^{\prime}}\left(\mu_{j}-k\right)>\sum_{j=1}^{\lambda_{k}^{\prime}}\left(\lambda_{j}-k\right) \geq$ $\sum_{j=1}^{\mu_{k}^{\prime}}\left(\lambda_{j}-k\right)$, thus $\mu \unlhd \lambda$, a contradiction.

(5.7) Stratification of the nilpotent variety. Recall that $\mathbb{C}$ is equipped with the usual metric topology, that subsets of topological spaces are equipped with induced topologies, and that direct products of topological spaces are equipped with product topologies. We consider the matrix algebra $\mathbb{C}^{n \times n}$ as a topological space. Then matrix addition and multiplication are continuous maps $\mathbb{C}^{n \times n} \times$ $\mathbb{C}^{n \times n} \rightarrow \mathbb{C}^{n \times n}$, as well as is scalar multiplication $\mathbb{C} \times \mathbb{C}^{n \times n} \rightarrow \mathbb{C}^{n \times n}$.

Recall that, for $M \in \mathbb{C}^{n \times n}$, the $\operatorname{rank} \operatorname{rk}(M) \in \mathbb{N}_{0}$ equals the smallest integer $k \in \mathbb{N}_{0}$ such that all $(i \times i)$-minors of $M$ vanish, for all $i \in\{k+1, \ldots, n\}$. This shows that $\mathcal{R}_{\leq k}:=\left\{M \in \mathbb{C}^{n \times n} ; \operatorname{rk}(M) \leq k\right\} \subseteq \mathbb{C}^{n \times n}$ is a closed subset, for all $k \in \mathbb{N}_{0}$. In particular, $\mathcal{G}:=\mathrm{GL}_{n}(\mathbb{C})=\mathbb{C}^{n \times n} \backslash \mathcal{R}_{\leq n-1}=\left\{A \in \mathbb{C}^{n \times n} ; \operatorname{det}(A) \neq\right.$ $0\} \subseteq \mathbb{C}^{n \times n}$ is an open subset, and since group multiplication $\mathcal{G} \times \mathcal{G} \rightarrow \mathcal{G}$ and inversion $\mathcal{G} \rightarrow \mathcal{G}: A \mapsto A^{-1}=\operatorname{det}(A)^{-1} \cdot \operatorname{adj}(A)$ are continuous maps, $\mathcal{G}$ becomes a topological group. Finally, $\mathcal{G}$ acts continuously on $\mathbb{C}^{n \times n}$ by conjugation $\mathcal{G} \times$ $\mathbb{C}^{n \times n} \rightarrow \mathbb{C}^{n \times n}:[A, M] \rightarrow A M A^{-1}$.

Let $\mathcal{N}:=\left\{M \in \mathbb{C}^{n \times n} ; M^{n}=0\right\}$ be the nilpotent variety; hence $\mathcal{N} \subseteq \mathbb{C}^{n \times n}$ is a closed subset. The group $\mathcal{G}$ acts continuously on $\mathcal{N}$ by conjugation, whose orbits are parametrised by the Jordan normal forms of matrices with respect to the eigenvalue 0 , that is block diagonal matrices $\bigoplus_{i=1}^{k} J_{\lambda_{i}} \in \mathcal{N}$, where $J_{\lambda_{i}} \in$ $\mathbb{C}^{\lambda_{i} \times \lambda_{i}}$ is a Jordan block of size $\lambda_{i} \in \mathbb{N}$. Permuting the Jordan blocks such that $\lambda_{1} \geq \cdots \geq \lambda_{k}$, from $\sum_{i=1}^{k} \lambda_{i}=n$ we infer that $\lambda:=\left[\lambda_{1}, \ldots, \lambda_{k}\right]$ is a partition of $n$ with $k \in\{0, \ldots, n\}$ parts, hence yielding the stratification $\mathcal{N}=\coprod_{\lambda \vdash n} \mathcal{N}_{\lambda}$, where $\mathcal{N}_{\lambda} \subseteq \mathcal{N}$ is the set of nilpotent matrices having Jordan normal form parametrised by $\lambda \vdash n$.

Since $\mathcal{G}$ acts continuously on $\mathcal{N}$ we conclude that, for any $\lambda \vdash n$, the closure $\overline{\mathcal{N}}_{\lambda} \subseteq \mathcal{N}$ of the $\mathcal{G}$-orbit $\mathcal{N}_{\lambda}$ is $\mathcal{G}$-invariant as well, thus is a union of $\mathcal{G}$-orbits. Hence we get an induced closure relation on the set $P(n)$ of all partitions of $n$, where for $\mu \vdash n$ we let $\mu \preceq \lambda$ if $\mathcal{N}_{\mu} \subseteq \overline{\mathcal{N}}_{\lambda}$. Hence $\preceq$ is reflexive, and since $\mathcal{N}_{\mu} \subseteq \overline{\mathcal{N}}_{\lambda}$ implies $\overline{\mathcal{N}}_{\mu} \subseteq \overline{\mathcal{N}}_{\lambda}$, it is transitive as well. Antisymmetry, saying that $\mathcal{N}_{\mu} \subseteq \overline{\mathcal{N}}_{\lambda}$ and $\mathcal{N}_{\lambda} \subseteq \overline{\mathcal{N}}_{\mu}$ already imply $\mathcal{N}_{\lambda}=\mathcal{N}_{\mu}$, is ensured as follows: By assumption both $\mathcal{N}_{\lambda}$ and $\mathcal{N}_{\mu}$ are dense in $\overline{\mathcal{N}}_{\lambda}=\overline{\mathcal{N}}_{\mu}$, hence the topological property that any $\mathcal{G}$-orbit is open in its closure entails that $\mathcal{N}_{\lambda} \cap \mathcal{N}_{\mu} \neq \emptyset$.

Actually, we are going to show that the closure relation $\preceq$ coincides with the 
dominance partial order $\unlhd$ on $P(n)$, by only using reflexivity and transitivity of $\preceq$, so that this will also imply that $\preceq$ indeed is a partial order:

For a Jordan block $J_{i} \in \mathbb{C}^{i \times i}$, for some $i \in \mathbb{N}$, we have $\operatorname{rk}\left(J_{i}^{k}\right)=i-k$ for all $k \in\{0, \ldots, i\}$. Thus for $M \in \mathcal{N}_{\lambda}$, where $\lambda=\left[n^{a_{n}}, \ldots, 1^{a_{1}}\right] \vdash n$, we have $\operatorname{rk}\left(M^{k}\right)=\sum_{i=k+1}^{n}(i-k) a_{i}=\sum_{i=k+1}^{n} \sum_{j=i}^{n} a_{j}=\sum_{i=k+1}^{n} \sum_{j=i}^{n}\left(\lambda_{i}^{\prime}-\lambda_{i+1}^{\prime}\right)=$ $\sum_{i=k+1}^{n} \lambda_{i}^{\prime}$, for all $k \in\{0, \ldots, n\}$, implying $n-\operatorname{rk}\left(M^{k}\right)=\sum_{i=1}^{k} \lambda_{i}^{\prime}$. Hence $\mathcal{N}_{\lambda}$ is uniquely determined by the rank sequence $\left[n-\sum_{i=1}^{k} \lambda_{i}^{\prime} \in \mathbb{N}_{0} ; k \in\{0, \ldots, n\}\right]$; note that we have $\operatorname{rk}\left(M^{0}\right)=n$ and $\operatorname{rk}\left(M^{n}\right)=0$ anyway.

Moreover, for $\mu \vdash n$ and $N \in \mathcal{N}_{\mu}$ we have $\mu \unlhd \lambda$ if and only if $\lambda^{\prime} \unlhd \mu^{\prime}$, which holds if and only if $\operatorname{rk}\left(M^{k}\right) \geq \operatorname{rk}\left(N^{k}\right)$, for all $k \in\{0, \ldots, n\}$. Thus we have $N \in \mathcal{N}_{\unlhd \lambda}:=\coprod_{\mu \unlhd \lambda} \mathcal{N}_{\mu} \subseteq \mathcal{N}$ if and only if $\operatorname{rk}\left(N^{k}\right) \leq n-\sum_{i=1}^{k} \lambda_{i}^{\prime}$, that is $N^{k} \in \mathcal{R}_{n-\sum_{i=1}^{k} \lambda_{i}^{\prime}}$, for all $k \in\{0, \ldots, n\}$. This implies that $\mathcal{N}_{\unlhd \lambda} \subseteq \mathcal{N}$ is a closed subset, containing $\mathcal{N}_{\lambda}$, thus we have $\overline{\mathcal{N}}_{\lambda} \subseteq \mathcal{N}_{\unlhd \lambda}$.

For the converse $\mathcal{N}_{\unlhd \lambda} \subseteq \overline{\mathcal{N}}_{\lambda}$, we have to show that $\mu \unlhd \lambda$ implies $\mathcal{N}_{\mu} \subseteq \overline{\mathcal{N}}_{\lambda}$. In order to do so, by the transitivity of the closure relation we may assume that $\mu:=\left[\lambda_{1}, \ldots, \lambda_{r-1}, \lambda_{r}-1, \lambda_{r+1}, \ldots, \lambda_{s-1}, \lambda_{s}+1, \lambda_{s+1}, \ldots, \lambda_{n}\right] \lessdot \lambda$, for some $1 \leq r<s \leq n$. Letting $a:=\lambda_{r}$ and $b:=\lambda_{s}$, hence $a>b \geq 0$, we have $J_{a} \oplus J_{b} \oplus N \in \mathcal{N}_{\lambda}$ and $J_{a-1} \oplus J_{b+1} \oplus N \in \mathcal{N}_{\mu}$, where $N \in \mathbb{C}^{(n-a-b) \times(n-a-b)}$. Hence we may assume that $\lambda=[a, b] \vdash n$ and $\mu=[a-1, b+1] \vdash n$, and let

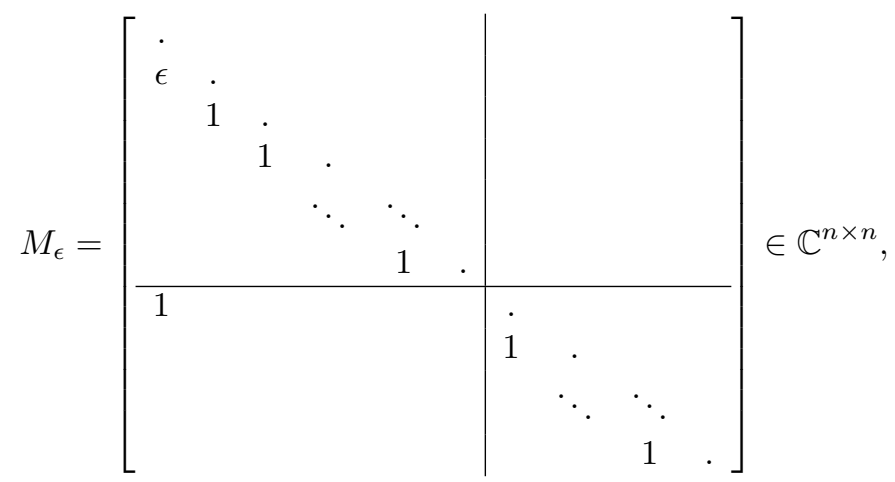

where the upper left and lower right hand corners have size $a \times a$ and $b \times b$, respectively. We show that $M_{\epsilon} \in \mathcal{N}_{\lambda}$ if $\epsilon \neq 0$, while $\lim _{\epsilon \rightarrow 0} M_{\epsilon}=M_{0} \in \mathcal{N}_{\mu}$ :

If $\epsilon \neq 0$ then, since $a>b$, the unit vector $e_{1} \in \mathbb{C}^{n \times 1}$ has minimum polynomial $X^{a} \in \mathbb{C}[X]$ with respect to $M_{\epsilon}$. Moreover, the unit vector $e_{a+1} \in \mathbb{C}^{n \times 1}$ has minimum polynomial $X^{b} \in \mathbb{C}[X]$; here for $b=0$ we let $e_{a+1}:=0 \in \mathbb{C}^{n \times 1}$. From $\left\langle e_{a+1}\right\rangle_{M_{\epsilon}}=\left\langle e_{a+1}, \ldots, e_{n}\right\rangle_{\mathbb{C}}$ and $\left\langle e_{1}\right\rangle_{M_{\epsilon}} \cap\left\langle e_{a+1}, \ldots, e_{n}\right\rangle_{\mathbb{C}}=\{0\}$ we conclude $\mathbb{C}^{n \times 1}=\left\langle e_{1}\right\rangle_{M_{\epsilon}} \oplus\left\langle e_{a+1}\right\rangle_{M_{\epsilon}}$, hence $M_{\epsilon}$ has Jordan normal form $J_{a} \oplus J_{b}$.

If $\epsilon=0$ then $e_{2} \in \mathbb{C}^{n \times 1}$ and $e_{1} \in \mathbb{C}^{n \times 1}$ have minimum polynomials $X^{a-1} \in$ $\mathbb{C}[X]$ and $X^{b+1} \in \mathbb{C}[X]$, respectively, with respect to $M_{0}$. From $\left\langle e_{2}\right\rangle_{M_{0}}=$ $\left\langle e_{2}, \ldots, e_{a}\right\rangle_{\mathbb{C}}$ and $\left\langle e_{1}\right\rangle_{M_{0}}=\left\langle e_{1}, e_{a+1}, \ldots, e_{n}\right\rangle_{\mathbb{C}}$ we conclude $\mathbb{C}^{n \times 1}=\left\langle e_{2}\right\rangle_{M_{0}} \oplus$ $\left\langle e_{1}\right\rangle_{M_{0}}$, hence $M_{0}$ has Jordan normal form $J_{a-1} \oplus J_{b+1}$. 


\section{Modular lattices}

(6.1) Modular lattices. The exposition is taken from on [19]. Let $X$ be a lattice, having a zero element $\underline{0} \in X$, which is locally chain-finite, that is all chains in any interval $[\underline{0}, x] \subseteq X$ are finite; in particular, in any interval $[x, y] \subseteq X$ there are saturated chains.

Then $X$ is called modular if for all $x, y, z \in X$ such that $z \leq x$ we have the modular law $x \wedge(y \vee z)=(x \wedge y) \vee z$. Then, for $x, y \in X$ the map $[x \wedge y, x] \rightarrow[y, x \vee y]: z \mapsto z \vee y$ is an isomorphism of lattices, with inverse $[y, x \vee y] \rightarrow[x \wedge y, x]: z \mapsto z \wedge x$ : Indeed, for $z \in[x \wedge y, x]$ we have $(z \vee y) \wedge x=$ $z \vee(y \wedge x)=z$, and for $z \in[y, x \vee y]$ we have $(z \wedge x) \vee y=z \wedge(x \vee y)=z$.

An element $z \in X \backslash\{\underline{0}\}$ is called join-irreducible or local, if whenever there are $x, y \in X$ such that $x \vee y=z$ then we already have $x=z$ or $y=z$. Hence $z \in X \backslash\{\underline{0}\}$ is join-irreducible if and only if $|\{y \in X ; y \lessdot z\}|=1$. Let $\mathcal{L} \subseteq X$ be the set of join-irreducible elements of $X$; note that $\mathcal{L}$ contains all atoms of $X$.

Similarly, if $\underline{1} \in X$ is a one element, an element $z \in X \backslash\{\underline{1}\}$ is called meetirreducible or co-local, if whenever there are $x, y \in X$ such that $x \wedge y=z$ then we already have $x=z$ or $y=z$. Hence $z \in X \backslash\{\underline{1}\}$ is meet-irreducible if and only if $|\{y \in X ; y \lessdot z\}|=1$. Let $\mathcal{L}^{*} \subseteq X$ be the set of meet-irreducible elements of $X$; note that $\mathcal{L}^{*}$ contains all co-atoms of $X$.

(6.2) Length and rank. a) Let $X$ be a modular lattice. Given an interval $[x, y] \subseteq X$, all saturated chains in $[x, y]$ have one and the same length; in particular, if $X$ has a one element, then $X$ is graded:

Let $x=x_{0} \lessdot x_{1} \lessdot \cdots \lessdot x_{s-1} \lessdot x_{s}=y$ and $x=x_{0}^{\prime} \lessdot x_{1}^{\prime} \lessdot \cdots \lessdot x_{t-1}^{\prime} \lessdot x_{t}^{\prime}=y$ be saturated chains, where $s, t \in \mathbb{N}_{0}$. Since $s=0$ if and only if $x=y$ if and only if $t=0$, and $s=1$ if and only if $x \lessdot y$ if and only if $t=1$, we may assume that $s \geq t \geq 2$, and proceed by induction on $s$. If $x_{s-1}=x_{t-1}^{\prime}$ then we have $s=t$ by induction. If $x_{s-1} \neq x_{t-1}^{\prime}$ then we have $x_{s-1} \vee x_{t-1}^{\prime}=y$, thus for $z:=x_{s-1} \wedge x_{t-1}^{\prime}$ we get $\left[z, x_{s-1}\right] \cong\left[x_{t-1}^{\prime}, y\right]$ and $\left[z, x_{t-1}^{\prime}\right] \cong\left[x_{s-1}, y\right]$, hence $z \lessdot x_{s-1}$ and $z \lessdot x_{t-1}^{\prime}$. Now extending a saturated chain $x=x_{0}^{\prime \prime}<x_{1}^{\prime \prime}<\cdots<x_{r-1}^{\prime \prime}<x_{r}^{\prime \prime}=z$ to $x_{s-1}$ and $x_{t-1}^{\prime}$, respectively, by induction we get $s-1=r+1=t-1$.

Hence there is a well-defined Jordan-Dedekind length map $l: X \rightarrow \mathbb{N}_{0}$ defined by $l(x):=l(\underline{0}, x)$ for $x \in X$. As in the case of graded posets, we have $l(\underline{0})=0$, and $l(y)=l(x)+1$ whenever $x \lessdot y \in X$; in particular we have $l(x)=0$ if and only if $x=\underline{0}$. Moreover, for $x \leq y \in X$ we have $l(x, y)=l(y)-l(x)$, and for $x, y \in X$ we have the modular equality $l(x \vee y)-l(y)=l(x)-l(x \wedge y)$.

b) Each element $x \in X$ is the join of finitely many join-irreducible elements.

For these representations of $x$ we have the following exchange property: Let $x=\bigvee_{i=1}^{r} x_{i}=\bigvee_{j=1}^{s} x_{j}^{\prime}$, where $r, s \in \mathbb{N}_{0}$ and $x_{i}, x_{j}^{\prime} \in \mathcal{L}$. Then for any $k \in$ $\{1, \ldots, r\}$ there is $j=j_{k} \in\{1, \ldots, s\}$ such that $x=x_{j}^{\prime} \vee \bigvee_{i \neq k} x_{i}$ :

Let $y:=\bigvee_{i \neq k} x_{i} \in X$, where we may assume that $y<x$. Hence we have $[y, x]=$ 
$\left[y, y \vee x_{k}\right] \cong\left[y \wedge x_{k}, x_{k}\right]$. Since $x_{k} \in \mathcal{L}_{\left[y \wedge x_{k}, x_{k}\right]}$, we infer that $x=y \vee x_{k} \in \mathcal{L}_{[y, x]}$ Now, for all $j \in\{1, \ldots, s\}$ we have $y \leq y \vee x_{j}^{\prime} \leq x$, hence from $x=\bigvee_{j=1}^{s}\left(y \vee x_{j}^{\prime}\right)$ we infer that there is $j \in\{1, \ldots, s\}$ such that $x=y \vee x_{j}^{\prime}$.

Hence we conclude that, if $x=\bigvee_{i=1}^{r} x_{i} \in X$ is irredundant, where $x_{i} \in \mathcal{L}$ for all $i \in\{1, \ldots, r\}$, then the number $r \in \mathbb{N}_{0}$ is independent of the particular choice of the irredundant representation, giving rise to the rank map $r: X \rightarrow \mathbb{N}_{0}$ :

Let $x=\bigvee_{i=1}^{r} x_{i}=\bigvee_{j=1}^{s} x_{j}^{\prime}$ be irredundant, where $r, s \in \mathbb{N}_{0}$ and $x_{i}, x_{j}^{\prime} \in \mathcal{L}$. Then iterating the above exchange procedure yields $x=\bigvee_{i=1}^{r} x_{j_{i}}^{\prime}$, hence the irredundancy of $\bigvee_{j=1}^{s} x_{j}^{\prime}$ entails $\left\{j_{1}, \ldots, j_{r}\right\}=\{1, \ldots, s\}$, hence $s \leq r$. Similarly we conclude $r \leq s$, hence equality holds.

Note that since $\underline{0}<x_{1}<x_{1} \vee x_{2}<\cdots<\bigvee_{i=1}^{r} x_{i}=x$ we have $r(x)=r \leq l(x)$, and that $r(x)=0$ if and only if $x=\underline{0}$.

(6.3) Complemented lattices. Let $X$ be a modular lattice having a one element 1 . Then $X$ is called complemented, if for all $x \in X$ there is a complement $y \in X$, that is we have $x \vee y=\underline{1}$ and $x \wedge y=\underline{0}$. Note that hence $X$ is complemented if and only if the dual of $X$ is.

a) The following assertions are equivalent: i) The lattice $X$ is complemented. ii) The one element $\underline{1} \in X$ is a join of atoms, that is $X$ is semi-simple. iii) Any element $x \in X$ is a join of atoms. iv) The set $\mathcal{L} \subseteq X$ of join-irreducible elements consists of (all) atoms.

This is seen as follows: In order to show i) $\Rightarrow \mathrm{ii}$ ), assume that $x:=\bigvee_{0 \lessdot z} z \neq \underline{1}$, and let $\underline{0} \neq y \in X$ be a complement of $x$. Hence there is an atom $\underline{0} \lessdot z \in X$ such that $z \leq y$, thus $x \wedge y \neq \underline{0}$, a contradiction.

Conversely, to show ii) $\Rightarrow \mathrm{i}$ ), let $x \in X$ and let $y \in X$ be maximal such that $x \wedge y=\underline{0}$. Assume that $x \vee y \neq \underline{1}$, thus there is an atom $\underline{0} \lessdot z \in X$ such that $z \not \leq(x \vee y)$, hence $z \wedge(x \vee y)=\underline{0}$. By the choice of $y$ we have $x \wedge(y \vee z) \neq \underline{0}$, and thus there is an atom $\underline{0} \lessdot w \in X$ such that $w \leq x \wedge(y \vee z)$. From $x \wedge y=\underline{0}$ we infer that $w \not \leq y$, hence since $[y, y \vee z] \cong[y \wedge z, z]=[\underline{0}, z]$ we conclude that $y \vee w=y \vee z$, entailing $x \vee y \vee z=x \vee y \vee w=x \vee y$, a contradiction.

Next, as for the implication i) $\Rightarrow$ iii), in view of the implication i) $\Rightarrow$ ii) applied to the interval $[\underline{0}, x] \subseteq X$, it suffices to show that all intervals $[x, y] \subseteq X$ are complemented: Indeed, let $x \leq z \leq y$, let $v \in X$ be a complement of $z$, and let $w:=(x \vee v) \wedge y=x \vee(v \wedge y) \in X$; hence we have $x \leq w \leq y$. This entails $z \wedge w=z \wedge(v \vee x)=(z \wedge v) \vee x=x$ and $z \vee w=z \vee(v \wedge y)=(z \vee v) \wedge y=y$ showing that $w$ is a complement of $z$ in $[x, y]$.

The implication iii) $\Rightarrow$ ii) is trivial. The implication iii) $\Rightarrow$ iv) follows from observing that only the atoms are join-irreducible. The implication iv) $\Rightarrow$ iii) follows from observing that any element of $X$ is a join of join-irreducible elements.

b) We give another characterisation, in terms of the length and rank functions: The lattice $X$ is complemented if and only if $r(\underline{1})=l(\underline{1}) \in \mathbb{N}_{0}$, which holds if 
and only if the rank and length functions of $X$ coincide:

The second assertion follows from the first one by recalling that if $X$ is complemented then all intervals of $X$ are as well. To show the first assertion, we proceed by induction on $r:=r(\underline{1}) \in \mathbb{N}_{0}$, the cases $r=0$ and $r=1$ being trivial, let $r \geq 2$. Let $\underline{1}=\bigvee_{i=1}^{r} x_{i} \in X$ be irredundant, where $x_{i} \in \mathcal{L}$ for all $i \in\{1, \ldots, r\}$, and let $x:=\bigvee_{i \neq j} x_{i} \in X$, for some $j \in\{1, \ldots, r\}$.

Now, if $X$ is complemented, then the interval $[\underline{0}, x] \subseteq X$ is as well, hence by induction we have $l(x)=r(x)=r-1$; since $\underline{0} \lessdot x_{j} \in X$ is an atom we have $x \wedge x_{j}=\underline{0}$, and hence $l\left(x_{j}\right)=1$ implies $r=l(x)+l\left(x_{j}\right)=l\left(x \vee x_{j}\right)+l\left(x \wedge x_{j}\right)=$ $l(\underline{1})+l(\underline{0})=l(\underline{1})$. If conversely $r=l(\underline{1})$, then we have $r-1=r(x) \leq l(x)<$ $l(\underline{1})=r$, thus $r(x)=l(x)=r-1$, and hence by induction the interval $[\underline{0}, x] \subseteq X$ is complemented, thus all the $x_{i} \in X$, for $i \neq j$, are atoms; since $j \in\{1, \ldots, r\}$ was arbitrary and $r \geq 2$, we conclude that $\underline{1} \in X$ is a join of atoms.

(6.4) The center. a) Let $X$ be a modular lattice. Then the center $\mathcal{Z}(X)$ of $X$ is defined as the set of all $x \in X$ such that there is $x^{\prime} \in X$ such that $X$ can be written is an inner direct product $X \cong[\underline{0}, x] \times\left[\underline{0}, x^{\prime}\right]$, that is the isomorphism is induced by the natural embeddings of the intervals $[\underline{0}, x]$ and $\left[\underline{0}, x^{\prime}\right]$ into $X$.

Then an element $z \in X$ decomposes into $z=z^{\prime} \vee z^{\prime \prime}$ for uniquely defined elements $\underline{0} \leq z^{\prime} \leq x$ and $\underline{0} \leq z^{\prime \prime} \leq x^{\prime}$. In particular, for $w \in \mathcal{L}$ we conclude that either $w=w^{\prime} \leq x$ and $w^{\prime \prime}=\underline{0}$, or $w=w^{\prime \prime} \leq x^{\prime}$ and $w^{\prime}=\underline{0}$. Hence we have $z=\bigvee\{w \in \mathcal{L} ; w \leq z\}=\bigvee\{w \in \mathcal{L} ; w \leq z \wedge x\} \vee \bigvee\left\{w \in \mathcal{L} ; w \leq z \wedge x^{\prime}\right\}=$ $(z \wedge x) \vee\left(z \wedge x^{\prime}\right)$, thus $z^{\prime}=z \wedge x$ and $z^{\prime \prime}=z \wedge x^{\prime}$. In other words, the inner decomposition is given by the isomorphism $X \rightarrow[\underline{0}, x] \times\left[\underline{0}, x^{\prime}\right]: z \mapsto\left[z \wedge x, z \wedge x^{\prime}\right]$.

In particular, given $x \in \mathcal{Z}(X)$, then $x^{\prime} \in X$ as above is uniquely defined: If similarly $X \cong[\underline{0}, x] \times\left[\underline{0}, x^{\prime \prime}\right]$ is an inner decomposition for some $x^{\prime \prime} \in X$, then since $x^{\prime} \wedge x=\underline{0}=x^{\prime \prime} \wedge x$ we have $x^{\prime \prime}=x^{\prime} \wedge x^{\prime \prime}=x^{\prime}$.

We have $\mathcal{Z}(X) \neq \emptyset$ if and only if $X$ has a one element $\underline{1}$. In this case, we have $\{\underline{0}, \underline{1}\} \subseteq \mathcal{Z}(X)$; and if $\mathcal{Z}(X)=\{\underline{0}, \underline{1}\}$, then $X$ is called indecomposable, otherwise $X$ is called decomposable.

b) Let $X$ have a one element $\underline{1}$. Then $\mathcal{Z}(X)$ gives rise to a canonical decomposition of $X$. In order to derive this, we show next that $\mathcal{Z}(X)$ is closed with respect to taking meets, that is if $x, y \in \mathcal{Z}(X)$ then we also have $x \wedge y \in \mathcal{Z}(X)$ :

Let $[\underline{0}, x] \times\left[\underline{0}, x^{\prime}\right] \cong X \cong[\underline{0}, y] \times\left[\underline{0}, y^{\prime}\right]$, where $x^{\prime}, y^{\prime} \in X$, and let $x_{11}:=x \wedge y$, $x_{12}:=x \wedge y^{\prime}, x_{21}:=x^{\prime} \wedge y$ and $x_{22}:=x^{\prime} \wedge y^{\prime}$. We consider the order-preserving maps $\sigma: \widehat{X}:=\prod_{i, j \in\{1,2\}}\left[\underline{0}, x_{i j}\right] \rightarrow X:\left[z_{i j} ; i, j \in\{1,2\}\right] \mapsto \bigvee_{i, j \in\{1,2\}} z_{i j}$ and $\tau: X \rightarrow \widehat{X}: z \mapsto\left[z \wedge x_{i j} ; i, j \in\{1,2\}\right]$.

For $z \in X$ we have $\bigvee_{i, j \in\{1,2\}}\left(z \wedge x_{i j}\right)=((z \wedge x) \wedge y) \vee\left((z \wedge x) \wedge y^{\prime}\right) \vee((z \wedge$ $\left.\left.x^{\prime}\right) \wedge y\right) \vee\left(\left(z \wedge x^{\prime}\right) \wedge y^{\prime}\right)=(z \wedge x) \vee\left(z \wedge x^{\prime}\right)=z$, hence $\sigma \tau=\mathrm{id}_{X}$. Conversely, for $\left[z_{i j} ; i, j \in\{1,2\}\right] \in \widehat{X}$ we have $\left(z_{12} \vee z_{21} \vee z_{22}\right) \wedge x_{11} \leq\left(x_{12} \vee x_{21} \vee x_{22}\right) \wedge$ $x_{11}=\left(x_{12} \vee x^{\prime}\right) \wedge(x \wedge y)=\left(x_{12} \vee\left(x^{\prime} \wedge x\right)\right) \wedge y=\left(x \wedge y^{\prime}\right) \wedge y=\underline{0}$, implying 
$\left(\bigvee_{i, j \in\{1,2\}} z_{i j}\right) \wedge x_{11}=z_{11} \vee\left(\left(z_{12} \vee z_{21} \vee z_{22}\right) \wedge x_{11}\right)=z_{11}$; similarly we argue for $x_{12}, x_{21}$ and $x_{22}$. Hence we have $\tau \sigma=\mathrm{id}_{\widehat{X}}$.

Thus $\sigma$ and $\tau$ are a pair of mutually inverse isomorphisms of lattices, showing that we have a decomposition $X \cong[\underline{0}, x \wedge y] \times\left[\underline{0}, x \wedge y^{\prime}\right] \times\left[\underline{0}, x^{\prime} \wedge y\right] \times\left[\underline{0}, x^{\prime} \wedge y\right]$, in particular we have $x \wedge y=x_{11} \in \mathcal{Z}(X)$.

Now, if $\left\{z_{1}, \ldots, z_{d}\right\}$, for some $d \in \mathbb{N}_{0}$, are the minimal elements of $\mathcal{Z}(X) \backslash\{\underline{0}\}$, then $X \cong \prod_{i=1}^{d}\left[\underline{0}, z_{i}\right]$ is the unique decomposition into non-trivial indecomposable intervals:

By the above, for $y \leq x \in \mathcal{Z}(X)$ we have the inner decomposition $[\underline{0}, x] \cong$ $[\underline{0}, y] \times\left[\underline{0}, x \wedge y^{\prime}\right]$, hence $y \in \mathcal{Z}([\underline{0}, x]) \subseteq \mathcal{Z}(X)$. Thus for $x \in \mathcal{Z}(X) \backslash\{\underline{0}\}$ the interval $[\underline{0}, x]$ is indecomposable if and only if $x$ is minimal in $\mathcal{Z}(X) \backslash\{\underline{0}\}$. Since for $x \in \mathcal{Z}(X)$ we have $l(\underline{1})=l(x)+l\left(x^{\prime}\right)$, by induction on $l(x)$ we conclude that $X \cong \prod_{i=1}^{d}\left[\underline{0}, z_{i}\right]$, for some $d \in \mathbb{N}_{0}$ and certain minimal $z_{i} \in \mathcal{Z}(X) \backslash\{\underline{0}\}$. Moreover, for any $x \in X$ we have $x=\bigvee_{i=1}^{d}\left(x \wedge z_{i}\right)$, hence if $x \in \mathcal{Z}(X) \backslash\{\underline{0}\}$ is minimal then we have $x=x \wedge z_{i}=z_{i}$ for a unique $i \in\{1, \ldots, d\}$, implying that $\left\{z_{1}, \ldots, z_{d}\right\}$ indeed encompasses all minimal elements of $\mathcal{Z}(X) \backslash\{\underline{0}\}$.

In particular we conclude that $\mathcal{Z}(X)$ is a finite complemented lattice: For any $x \in \mathcal{Z}(X)$ we have $[\underline{0}, x] \cong \prod_{i=1}^{d}\left[\underline{0}, x \wedge z_{i}\right] \cong \prod_{i ; z_{i} \leq x}\left[\underline{0}, z_{i}\right]$, thus $x=\bigvee\left\{z_{i} ; z_{i} \leq\right.$ $x$ \}, showing that $x$ is the join of certain of the finitely many atoms in $\mathcal{Z}(X)$. $\sharp$

(6.5) Radicals. a) Let $X$ be a modular lattice. For $\underline{0} \neq x \in X$ the element $x_{*}:=\bigwedge\{y \in X ; y \lessdot x\} \in X$ is called the radical of $x$; we let $\underline{0}_{*}:=\underline{0}$. In particular, we have $\mathcal{L}=\left\{x \in X ; x_{*} \lessdot x\right\}$. We collect a few properties:

i) For $x \in X$, the radical $x_{*}$ is small in $x$, that is for $y \leq x \in X$ such that $y \vee x_{*}=x$ we already have $y=x$ : Indeed, assuming that $y<x$, there is $y \leq z \lessdot x$, hence $y \vee x_{*} \leq z$, a contradiction.

ii) We have $x_{*}=\bigwedge\{y \in X ;[y, x]$ complemented $\}$ : We may assume that $x \neq \underline{0}$. From $x_{*}=\bigwedge\{z \in X ; z \lessdot x\}$, considering the dual of the interval $\left[x_{*}, x\right]$, we infer that $\left[x_{*}, x\right]$ is complemented, hence $x_{*} \subseteq \bigwedge\{y \in X ;[y, x]$ complemented $\}$. Conversely, if for $y \leq x \in X$ the interval $[y, x]$ is complemented, then again considering duals, we have $x_{*}=\bigwedge\{z \in X ; z \lessdot x\} \leq \bigwedge\{z \in X ; y \leq z \lessdot x\}=y$.

iii) We have $r(x)=r_{\left[x_{*}, x\right]}(x)$ : Let $x=\bigvee_{i=1}^{r} x_{i}$ be irredundant, where $r=$ $r(x) \in \mathbb{N}_{0}$ and $x_{i} \in \mathcal{L}$. Hence $x_{i} \not \leq x_{*}$, and since $\left[x_{*}, x_{i} \vee x_{*}\right] \cong\left[x_{i} \wedge x_{*}, x_{i}\right]$ is complemented, we have $x_{*} \lessdot x_{i} \vee x_{*}$, that is $x_{i} \vee x_{*} \in \mathcal{L}_{\left[x_{*}, x\right]}$. Assume that $x=\bigvee_{i \in \mathcal{I}}\left(x_{i} \bigvee x_{*}\right)$, for some $\mathcal{I} \subset\{1, \ldots, r\}$, then we also have $x=\bigvee_{i \in \mathcal{I}} x_{i}$, a contradiction. Thus $x=\bigvee_{i=1}^{r}\left(x_{i} \vee x_{*}\right)$ is irredundant in $\left[x_{*}, x\right]$, hence $r_{\left[x_{*}, x\right]}(x)=r$.

iv) Finally, for $x, y \in X$ we have $(x \vee y)_{*}=x_{*} \vee y_{*}$ : Let $x=\bigvee_{i=1}^{r} x_{i}$ and $y=\bigvee_{j=1}^{s} y_{j}$ be irredundant, where $r, s \in \mathbb{N}_{0}$ and $x_{i}, y_{j} \in \mathcal{L}$. Since $\left[x_{*} \vee y_{*}, x_{i} \vee\right.$ $\left.\left(x_{*} \vee y_{*}\right)\right] \cong\left[x_{i} \wedge\left(x_{*} \vee y_{*}\right), x_{i}\right]$ is complemented, we have either $x_{i} \leq x_{*} \vee y_{*}$ or $x_{*} \vee y_{*} \lessdot x_{i} \vee\left(x_{*} \vee y_{*}\right)$. A similar statement holds for the $y_{j}$, and hence $x \vee y$ is a join of atoms of $\left[x_{*} \vee y_{*}, x \vee y\right]$. Thus we have $(x \vee y)_{*} \leq x_{*} \vee y_{*}$. 
Conversely, assume that $x_{*} \not \leq(x \vee y)_{*}$. Then $\left[(x \vee y)_{*} \wedge x, x\right] \cong\left[(x \vee y)_{*}, x \vee\right.$ $\left.(x \vee y)_{*}\right]$ is not complemented. Since $(x \vee y)_{*} \leq x \vee(x \vee y)_{*} \leq x \vee y$, this a contradiction. Hence we have $x_{*} \leq(x \vee y)_{*}$, and similarly $y_{*} \leq(x \vee y)_{*}$. \#

b) The above considerations lead to the following subsets of $X$ : For $r \in \mathbb{N}_{0}$ let $X_{r}:=\{x \in X ; r(x)=r\}=\left\{x \in X ; r_{\left[x_{*}, x\right]}(x)=r\right\} \subseteq X$ and $\mathcal{L}_{r}:=\{x \in$ $X_{r} ;\left[x_{*}, x\right]$ indecomposable $\} \subseteq X_{r}$. Hence in particular we have $X_{0}=\mathcal{L}_{0}=\{\underline{0}\}$ and $X_{1}=\mathcal{L}_{1}=\mathcal{L}$.

\section{Benson-Conway Theorem}

We have now presented the general theory of modular lattices needed to proceed towards the main result of this section, given in (7.3). We need a few more specially tailored notions, inspired by [14]:

(7.1) Dotted-lines. a) Let $X$ be a modular lattice. For an element $z \in X$ we have $z \in X_{2}$, that is $r(z)=2$, if and only if there are $x_{1}, x_{2} \in \mathcal{L}$ such that $x_{1} \not \leq x_{2} \not \leq x_{1}$ and $z=x_{1} \vee x_{2}$.

In this case, we have $l_{\left[z_{*}, z\right]}(z)=r_{\left[z_{*}, z\right]}(z)=2$. Hence the elements of $\left[z_{*}, z\right]$ are its zero element $z_{*}$, its one element $z$, and its atoms $z_{*} \lessdot z_{i} \lessdot z$, for $i \in \mathcal{I}_{z}$, where $\mathcal{I}_{z}$ is a suitable index set. In particular, since $z_{*}<z$ is small, for $z_{i}:=x_{i} \vee z_{*} \in X$ we have $z_{*} \lessdot z_{i} \lessdot z$ and $z_{1} \neq z_{2}$, hence $\left|\mathcal{I}_{z}\right| \geq 2$.

If $\left|\mathcal{I}_{z}\right|=2$, then we have $\left[z_{*}, z\right] \cong\left[z_{*}, z_{1}\right] \times\left[z_{*}, z_{2}\right]$, where $z_{1} \neq z_{*} \neq z_{2}$, hence $\left[z_{*}, z\right]$ is decomposable. Conversely, let $\left[z_{*}, z\right] \cong\left[z_{*}, z^{\prime}\right] \times\left[z_{*}, z^{\prime \prime}\right]$, where $z^{\prime} \neq z_{*} \neq z^{\prime \prime}$. Since $l_{\left[z_{*}, z\right]}(z)=2$, we have $l_{\left[z_{*}, z\right]}\left(z^{\prime}\right)=l_{\left[z_{*}, z\right]}\left(z^{\prime \prime}\right)=1$, hence both $z_{*} \lessdot z^{\prime} \lessdot z$ and $z_{*} \lessdot z^{\prime \prime} \lessdot z$, and thus $\left|\mathcal{I}_{z}\right|=2$. Hence we conclude that $\left[z_{*}, z\right]$ is indecomposable if and only if $\left|\mathcal{I}_{z}\right| \geq 3$, and we have $\mathcal{L}_{2}=\left\{z \in X_{2} ;\left|\mathcal{I}_{z}\right| \geq 3\right\}$.

Now, given $z \in \mathcal{L}_{2}$, a set $\mathcal{D}=\left\{x_{i} \in \mathcal{L} ; i \in \mathcal{I}_{z}\right\}$, such that $x_{i} \vee z_{*}=z_{i} \in X$ for all $i \in \mathcal{I}_{z}$, is called a dotted-line for $z$; in particular, the $x_{i} \in \mathcal{D}$ are pairwise distinct and thus $|\mathcal{D}|=\left|\mathcal{I}_{z}\right| \geq 3$. Actually, dotted-lines always exist: Since each $z_{i}$ is the join of the join-irreducible elements it contains, and since $z_{*} \lessdot z_{i}$, we may choose $x_{i} \in \mathcal{L}$ such that $x_{i} \leq z_{i}$ and $x_{i} \not \leq z_{*}$, entailing $x_{i} \vee z_{*}=z_{i}$.

b) We collect a few properties of dotted-lines: Let $\mathcal{D}=\left\{x_{i} \in \mathcal{L} ; i \in \mathcal{I}_{z}\right\}$ be a dotted-line for $z \in \mathcal{L}_{2}$. Then we have $x_{i} \not \leq x_{j}$; moreover, we have $x_{i} \vee x_{j}=z$, for all $i \neq j \in \mathcal{I}_{z}$, and $\mathcal{D} \subseteq \mathcal{L}$ is maximal having this property. In particular, $\bigvee \mathcal{D}=z$ is well-defined, even if $\mathcal{D}$ is infinite.

For all $i \in \mathcal{I}_{z}$ let $z_{i}=x_{i} \vee z_{*} \lessdot z$. As $z_{i} \not \leq z_{j}$, for all $i \neq j \in \mathcal{I}_{z}$, we also have $x_{i} \not \leq x_{j}$. Moreover, since $z=z_{i} \vee z_{j}=x_{i} \vee x_{j} \vee z_{*}$ we have $x_{i} \vee x_{j}=z$.

Assume that there is $x_{0} \in \mathcal{L} \backslash \mathcal{D}$ such that $x_{0} \vee x_{i}=z$ for all $i \in \mathcal{I}_{z}$, and let $z_{0}:=x_{0} \vee z_{*}$. Assuming $z_{0}=z_{*}$ yields $z=x_{0} \vee x_{i}=x_{i} \vee z_{*}=x_{i}$, for all $i \in \mathcal{I}_{z}$, a contradiction; and assuming $z_{0}=z$ yields $z=x_{0} \vee z_{*}=x_{0}$, a contradiction. Hence we conclude that $z_{*} \lessdot z_{0} \lessdot z$. Thus there is $i \in \mathcal{I}_{z}$ such that $z_{0}=z_{i}$, hence $x_{0} \vee z_{*}=x_{i} \vee z_{*}$. This entails $z=x_{0} \vee x_{i} \vee z_{*}=x_{0} \vee z_{*}=x_{0}$, a contradiction. \# 
c) We proceed to give a characterisation of dotted-lines, which actually is the original definition given in [14]: Let $\mathcal{D}=\left\{x_{i} \in \mathcal{L} ; i \in \mathcal{I}\right\} \subseteq \mathcal{L}$, where $\mathcal{I}$ is an index set such that $|\mathcal{I}| \geq 3$, the $x_{i} \in \mathcal{L}$ are pairwise distinct such that $x_{i} \vee x_{j} \in X$ is independent of the choice of $i \neq j \in \mathcal{I}$, and $\mathcal{D} \subseteq \mathcal{L}$ is maximal having this property. Then $z:=\bigvee \mathcal{D} \in \mathcal{L}_{2} \subseteq X$ is well-defined and $\mathcal{D}$ is a dotted-line for $z$ :

Assume that we have $x_{i}<x_{j}$, for some $i \neq j \in \mathcal{I}$, and let $k \in \mathcal{I}$ such that $i \neq k \neq j$. Hence we have $x_{i} \vee x_{k}=x_{i} \vee x_{j}=x_{j} \in \mathcal{L}$, implying that $x_{i}=x_{j}$ or $x_{k}=x_{j}$, a contradiction. Thus we have $x_{i} \not \leq x_{j}$ for all $i \neq j \in \mathcal{I}$. Hence for $z:=x_{i} \vee x_{j}=\bigvee \mathcal{D}$ we have $r(z)=l_{\left[z_{*}, z\right]}(z)=2$, that is $z \in X_{2}$.

Let $z_{i}:=x_{i} \vee z_{*}$ for all $i \in \mathcal{I}$. Assuming $z_{i}=z_{*}$ yields $z=x_{i} \vee x_{j}=x_{j} \vee z_{*}=x_{j}$, for all $i \neq j \in \mathcal{I}$, a contradiction; and assuming $z_{i}=z$ yields $z=x_{i} \vee z_{*}=x_{i}$, a contradiction. Hence we conclude that $z_{*} \lessdot z_{i} \lessdot z$. Assume that $z_{i}=z_{j}$ for some $i \neq j \in \mathcal{I}$, then we have $z=x_{i} \vee x_{j} \vee z_{*}=z_{i} \vee z_{j}=z_{i}$, a contradiction. Thus we have $\mathcal{I} \subseteq \mathcal{I}_{z}$, and hence $z \in \mathcal{L}_{2}$.

Assume that there is $k \in \mathcal{I}_{z} \backslash \mathcal{I}$, and choose $x_{k} \in \mathcal{L}$ such that $z_{k}=x_{k} \vee z_{*}$. Hence for all $i \in \mathcal{I}$ we have $x_{k} \neq x_{i}$, and $x_{i} \vee x_{k} \vee z_{*}=z_{i} \vee z_{k}=z$ and thus $x_{i} \vee x_{k}=z$, contradicting the maximality property. Hence we have $\mathcal{I}=\mathcal{I}_{z}$. $\sharp$

(7.2) Completeness. a) Let $X$ be a modular lattice, and let $\mathcal{X} \subseteq \mathcal{L}$ be an ideal. Then $\mathcal{X}$ is called complete, if $\mathcal{X}$ has an upper bound in $X$, and for each dotted-line $\mathcal{D} \subseteq \mathcal{L}$, for any $z \in \mathcal{L}_{2}$, fulfilling $|\mathcal{D} \cap \mathcal{X}| \geq 2$ we already have $\mathcal{D} \subseteq \mathcal{X}$.

Let $X(\mathcal{L})$ be the partially ordered set of complete ideals of $\mathcal{L}$, where the partial order is given by set-theoretic inclusion. Hence $X(\mathcal{L})$ is closed under taking settheoretic intersections, and thus becomes a lattice by letting $\mathcal{X} \wedge \mathcal{X}^{\prime}:=\mathcal{X} \cap \mathcal{X}^{\prime}$ and $\mathcal{X} \vee \mathcal{X}^{\prime}:=\bigwedge\left\{\mathcal{Y} \in X(\mathcal{L}) ; \mathcal{X} \cup \mathcal{X}^{\prime} \subseteq \mathcal{Y}\right\} \in X(\mathcal{L})$. Moreover, by the local chain-finiteness of $X$, an ideal of $\mathcal{L}$ having a bound in $X$ has only finite chains, hence $X(\mathcal{L})$ is locally chain-finite as well.

b) For each $z \in \mathcal{L}_{2}$ choose a dotted-line $\mathcal{D}_{z} \subseteq \mathcal{L}$ for $z$. Then an ideal $\mathcal{X} \subseteq \mathcal{L}$ is called weakly complete with respect to $\left\{\mathcal{D}_{z} ; z \in \mathcal{L}_{2}\right\}$, if $\mathcal{X}$ has an upper bound in $X$, and for each $z \in \mathcal{L}_{2}$ such that $\left|\mathcal{D}_{z} \cap \mathcal{X}\right| \geq 2$ we already have $\mathcal{D}_{z} \subseteq \mathcal{X}$.

The notion of weak completeness is due to the observation that in general there is more than one dotted-line for a given element of $\mathcal{L}_{2}$; typically there are many, for example in the applications in [18]. We will show in (7.4) that in order to decide whether a given ideal of $\mathcal{L}$ is complete, it suffices to check for weak completeness, reducing considerably the necessary amount of checking.

c) If $X$ is finite, then the complete ideals of $\mathcal{L}$ are found as follows: The principal ideals of $\mathcal{L}$, being in bijection with $\mathcal{L}$, are complete. Taking these as initialisation, we iterate the following procedure: Picking in turn any of the complete ideals already found, we add a further ideal generator from $\mathcal{L}$, and then determine the smallest complete overideal of $\mathcal{L}$, by iteratively completing with respect to dotted-lines and the ideal property.

For example, let $X$ be the modular lattice whose Hasse diagram is depicted in 
Table 10: A strange modular lattice.

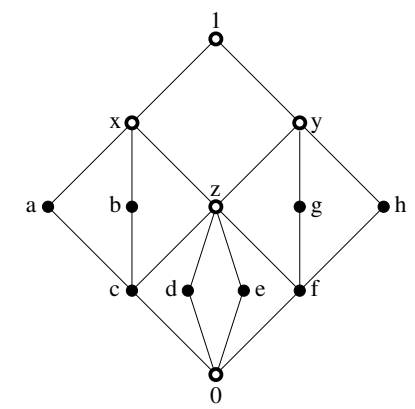

Table 10: We have $\mathcal{L}=\{a, b, c, d, e, f, g, h\}$ and $\mathcal{L}_{2}=\{x, y, z\}$ and $X_{2} \backslash \mathcal{L}_{2}=\{\underline{1}\}$, where $\left|\mathcal{I}_{x}\right|=3=\left|\mathcal{I}_{y}\right|$ and $\left|\mathcal{I}_{z}\right|=4$. The unique dotted-line for $z$ is $\{c, d, e, f\}$, while $x$ has three dotted-lines $\{\{a, b, d\},\{a, b, e\},\{a, b, f\}\}$, and $y$ also has three dotted-lines $\{\{c, g, h\},\{d, g, h\},\{e, g, h\}\}$. Apart from the principal ideals of $\mathcal{L}$, there are precisely the complete ideals

$$
\emptyset,\langle\leq c, d, e, f\rangle,\langle\leq a, b, d, e, f\rangle,\langle\leq c, d, e, g, h\rangle,\langle\leq a, b, g, h\rangle,
$$

where $\langle\leq S\rangle$ denotes the ideal generated by $S \subseteq \mathcal{L}$; here, it suffices to consider the ideals generated by two elements.

(7.3) Theorem: Benson-Conway [1985]. Let $X$ be a modular lattice. Then the following maps are a pair of mutually inverse isomorphisms of lattices:

$$
\beta: X \rightarrow X(\mathcal{L}): x \mapsto\{y \in \mathcal{L} ; y \leq x\} \quad \text { and } \quad \beta^{-1}: X(\mathcal{L}) \rightarrow X: \mathcal{X} \mapsto \bigvee \mathcal{X}
$$

Proof. The maps $\beta$ and $\beta^{-1}$ are well-defined and order-preserving, and we have $\beta^{-1} \circ \beta=\mathrm{id}_{X}$. Hence we have to show that $\beta \circ \beta^{-1}=\mathrm{id}_{X(\mathcal{L})}$ also holds:

Assume to the contrary that there are $\mathcal{X} \in X(\mathcal{L})$ and $y \in \mathcal{L} \backslash \mathcal{X}$ such that $y \leq \bigvee \mathcal{X}$. Let $n:=\min \{|\mathcal{Y}| \in \mathbb{N} ; \mathcal{Y} \subseteq \mathcal{X}$ finite, $y \leq \bigvee \mathcal{Y}\}$; note that $n \in \mathbb{N}$ is well-defined. Assume that $n=1$, then we have $y \leq x$ for some $x \in \mathcal{X}$, and since $\mathcal{X} \subseteq \mathcal{L}$ is an ideal we infer $y \in \mathcal{X}$, a contradiction. Hence we have $n \geq 2$, and we may choose $y$ such that $n$ is minimal. Let $\mathcal{Y}=\left\{y_{1}, \ldots, y_{n}\right\} \subseteq \mathcal{X}$, where we may choose $\mathcal{Y}$ such that $y \vee y_{1} \in X$ is minimal.

By modularity we have $y \vee y_{1}=\left(y \vee y_{1}\right) \wedge \bigvee_{i=1}^{n} y_{i}=\left(y \vee y_{1}\right) \wedge\left(y_{1} \vee \bigvee_{i=2}^{n} y_{i}\right)=$ $y_{1} \vee\left(\left(y \vee y_{1}\right) \wedge \bigvee_{i=2}^{n} y_{i}\right) \in X$. Let $z:=\left(y \vee y_{1}\right) \wedge \bigvee_{i=2}^{n} y_{i} \in X$, and let $z_{1}, \ldots, z_{r} \in \mathcal{L}$ such that $z=\bigvee_{j=1}^{r} z_{j} \in X$, for some $r \in \mathbb{N}_{0}$. Assume that $z_{j} \notin \mathcal{X}$ for some $j \in\{1, \ldots, r\}$, then we have $z_{j} \leq y \vee y_{1} \leq \bigvee \mathcal{X}$ and $z_{j} \leq \bigvee_{i=2}^{n} y_{i}$, contradicting the minimality of $n$. Hence we have $z_{j} \in \mathcal{X}$ for all $j \in\{1, \ldots, r\}$. 
By definition we have $y \vee y_{1}=y_{1} \vee z=y_{1} \vee \bigvee_{j=1}^{r} z_{j}$, hence since $y \not \leq y_{1}$ the exchange property entails $y \vee y_{1}=y_{1} \vee z_{j}$, for some $j \in\{1, \ldots, r\}$. In particular we have $y \leq y_{1} \vee z_{j}$, and thus $n=2$ and $y_{1} \neq z_{j}$. Moreover, since $z_{j} \leq y \vee y_{1}$ we have $y \vee z_{j} \leq y \vee y_{1}$, and by the minimality of $y \vee y_{1}$ we conclude that $y \vee z_{j}=y \vee y_{1}$. Hence, by the maximality property of dotted-lines, there is a dotted-line $\mathcal{D}$ such that $\left\{y, y_{1}, z_{j}\right\} \subseteq \mathcal{D}$. Since both $y_{1}, z_{j} \in \mathcal{X}$, completeness implies that $y \in \mathcal{X}$, the final contradiction.

(7.4) Theorem. Let $X$ be a modular lattice, and for each $z \in \mathcal{L}_{2}$ we pick a dotted-line $\mathcal{D}_{z} \subseteq \mathcal{L}$ for $z$. Then an ideal $\mathcal{X} \subseteq \mathcal{L}$ is complete if and only if it is weakly complete with respect to $\left\{\mathcal{D}_{z} ; z \in \mathcal{L}_{2}\right\}$.

Proof. We only have to show that a weakly complete ideal $\mathcal{X} \subseteq \mathcal{L}$ with respect to $\left\{\mathcal{D}_{z} ; z \in \mathcal{L}_{2}\right\}$ is already complete with respect to all dotted-lines $\mathcal{D} \subseteq \mathcal{L}$. To do so, we proceed by induction on $l(\bigvee \mathcal{X})$. If $l(\bigvee \mathcal{X}) \leq 1$, then $|\mathcal{X}| \leq 1$, thus $\mathcal{X}$ is complete. Hence let $l(\bigvee \mathcal{X}) \geq 2$, and let $x_{1}, x_{2} \in \mathcal{X} \cap \mathcal{D}$ such that $z:=x_{1} \vee x_{2} \in \mathcal{L}_{2}$. We show that for all $x \in \mathcal{L}$ such that $x \leq z$ we have $x \in \mathcal{X}$ :

For $i \in\{1,2\}$ let $z_{i}:=x_{i} \vee z_{*}$. Assuming $z_{i} \leq z_{*}$ yields $z=x_{1} \vee x_{2} \vee z_{*}=$ $z_{1} \vee z_{2}=x_{3-i} \vee z_{*}=x_{3-i}$, a contradiction; and assuming $z_{i}=z$ yields $z=$ $x_{i} \vee z_{*}=x_{i}$, a contradiction. Hence we have $z_{*} \lessdot z_{i} \lessdot z$. We consider the ideals $\mathcal{Z}_{*}:=\left\{y \in \mathcal{X} ; y \leq z_{*}\right\}$ and $\mathcal{Z}_{i}:=\left\{y \in \mathcal{X} ; y \leq z_{i}\right\}$. Since $\mathcal{X}$ is an ideal and $z_{*}=\left(x_{1}\right)_{*} \bigvee\left(x_{2}\right)_{*}$, we have $\bigvee \mathcal{Z}_{*}=z_{*}$, and hence we have $\bigvee \mathcal{Z}_{i}=z_{i} \lessdot z \leq \bigvee \mathcal{X}$.

For any distinguished dotted-line $\mathcal{D}_{w} \subseteq \mathcal{L}$, for $w \in \mathcal{L}_{2}$, such that $\left|\mathcal{D}_{w} \cap \mathcal{Z}_{i}\right| \geq 2$ we have $w=\bigvee \mathcal{D}_{w} \leq z_{i}$, and by weak completeness we have $\mathcal{D}_{w} \subseteq \mathcal{X}$, hence we conclude that $\mathcal{D}_{w} \subseteq \mathcal{Z}_{i}$. Thus $\mathcal{Z}_{i}$ is weakly complete, and since $l\left(\bigvee \mathcal{Z}_{i}\right)<l(\bigvee \mathcal{X})$ it is complete by induction.

Let $y_{i} \in \mathcal{D}_{z}$ such that $z_{i}=y_{i} \vee z_{*}$. Since the $\mathcal{Z}_{i}$ are complete such that $\bigvee \mathcal{Z}_{i}=z_{i}$, by (7.3) we have $y_{i} \in \mathcal{Z}_{i} \subseteq \mathcal{X}$, and as $\mathcal{X}$ is weakly complete we have $\mathcal{D}_{z} \subseteq \mathcal{X}$. Now we consider $k \in \mathcal{I}_{z}$ such that $x \leq z_{k} \lessdot z$, and let $\mathcal{Z}_{k}:=\left\{y \in \mathcal{X} ; y \leq z_{k}\right\}$. Since we have $\mathcal{D}_{z} \subseteq \mathcal{X}$ and $\bigvee \mathcal{Z}_{*}=z_{*}$, we obtain $\bigvee \mathcal{Z}_{k}=z_{k} \lessdot z \leq \bigvee \mathcal{X}$. Moreover, $\mathcal{Z}_{k}$ is weakly complete, and since $l\left(\bigvee \mathcal{Z}_{k}\right)<l(\bigvee \mathcal{X})$ it is complete by induction. As $\bigvee \mathcal{Z}_{k}=z_{k}$, by (7.3) we finally have $x \in \mathcal{Z}_{k} \subseteq \mathcal{X}$.

(7.5) Distributive lattices. a) Let $X$ be a locally chain-finite lattice, having a zero element $\underline{0} \in X$. Then $X$ is called distributive if the distributive law $x \wedge(y \vee z)=(x \wedge y) \vee(x \wedge z)$ holds for all $x, y, z \in X$. In this case any interval in $X$ is distributive as well. Note that for $z \leq x$ from the distributive law we get $x \wedge(y \vee z)=(x \wedge y) \vee z$, that is the modular law is fulfilled automatically, hence $X$ is modular.

Here is the natural example for a lattice fulfilling the distributive law: Let $N$ be a set. Then $\mathcal{P}(N)$, partially ordered by set-theoretic inclusion $\subseteq$ and having the zero element $\emptyset$, becomes a lattice with meet $M \cap M^{\prime}$ and join $M \cup M^{\prime}$, where for $M_{0}, M_{1}, M_{2} \subseteq N$ we have $M_{0} \cap\left(M_{1} \cup M_{2}\right)=\left(M_{0} \cap M_{1}\right) \cup\left(M_{0} \cap M_{2}\right)$. But note that $\mathcal{P}(N)$ is locally chain-finite if and only if $N$ is finite. 
b) The basic structure theorem for distributive lattices is Birkhoff's Representation Theorem, which is a special case of (7.3), shedding some further light on the significance of the set $\mathcal{L}_{2}$ :

Let $X$ be a modular lattice. Then $X$ is distributive if and only if $\mathcal{L}_{2}=\emptyset$. In this case, we have $X(\mathcal{L})=\{\mathcal{X} \subseteq \mathcal{L} ; \mathcal{X}$ finite ideal $\}$, and the following maps are a pair of mutually inverse isomorphisms of lattices:

$$
\beta: X \rightarrow X(\mathcal{L}): x \mapsto\{y \in \mathcal{L} ; y \leq x\} \quad \text { and } \quad \beta^{-1}: X(\mathcal{L}) \rightarrow X: \mathcal{X} \mapsto \bigvee \mathcal{X}
$$

Moreover, the length function on $X(\mathcal{L})$ is given by $l(\mathcal{X}):=|\mathcal{X}|$, and any interval $[\underline{0}, x]$, for $x \in X$, is finite. In particular, if $X$ has a one element, then $X$ is finite.

This is seen as follows: If $z \in \mathcal{L}_{2} \neq \emptyset$, then let $z_{*} \lessdot z_{i} \lessdot z$, for $i \in\{0,1,2\}$. Then we have $z_{0} \wedge\left(z_{1} \vee z_{2}\right)=z_{0} \neq z_{*}=\left(z_{0} \wedge z_{1}\right) \vee\left(z_{0} \wedge z_{2}\right)$, hence $\left[z_{*}, z\right]$ is not distributive, hence neither $X$ is. Conversely, if $\mathcal{L}_{2}=\emptyset$ then $X(\mathcal{L})$ is the set of ideals of $\mathcal{L}$ having a bound in $X$, and whose join and meet operations are given by taking set theoretic unions and intersections, respectively, hence $X(\mathcal{L})$ is distributive, and thus $X$ also is.

Now, letting $X$ be distributive, in order to determine the length function on $X(\mathcal{L})$, we show that $|\beta(x)|=l(x)$, for $x \in X$. We proceed by induction on $l:=l(x) \in \mathbb{N}_{0}$; the case $l=0$ being trivial, we assume that $l \geq 1$, and let $\mathcal{X}:=$ $\beta(x) \in X(\mathcal{L})$. Then there are $y \in X$ and $z \in \mathcal{L}$, such that $y \lessdot x$ and $x=y \vee z$. Letting $\mathcal{Y}:=\beta(y) \in X(\mathcal{L})$, and $\mathcal{Z}:=\beta(z)=\langle\leq z\rangle \in X(\mathcal{L})$ be the principal ideal generated by $z$, we get $\mathcal{X}=\mathcal{Y} \cup \mathcal{Z}$. We have $l(y)=l(x)-1$, and thus by induction we get $|\mathcal{Y}|=l(y)$. Moreover, we have $[y \wedge z, z] \cong[y, y \vee z]=[y, x]$, implying that $y \wedge z=z_{*} \lessdot z$, in other words $\mathcal{Z} \backslash\{z\}=\beta\left(z_{*}\right)=\beta(y \wedge z)=\mathcal{Y} \cap \mathcal{Z}$. This yields $\mathcal{Z}=(\mathcal{Y} \cap \mathcal{Z}) \dot{\cup}\{z\}$, and thus $\mathcal{X}=\mathcal{Y} \cup \mathcal{Z}=\mathcal{Y} \cup\{z\}$, saying that $|\mathcal{X}|=|\mathcal{Y}|+1=l(y)+1=l(x)$.

In particular this shows that $X(\mathcal{L})$ consists of the finite ideals of $\mathcal{L}$. Finally, since for any $x \in X$ the ideal $\beta(x) \subseteq \mathcal{L}$ is finite, there are only finitely many subideals of $\beta(x)$, in other words there are only finitely many $y \in X$ such that $y \leq x$, that is the interval $[\underline{0}, x]$ is finite.

(7.6) The block graph. Let $X$ be a modular lattice having a one element $\underline{1}$, and let $\beta$ be as in (7.3). We proceed to derive a description of the centre of $X$ :

a) For $x \in X$ let $\mathcal{X}:=\beta(x) \in X(\mathcal{L})$. Then we have $x \in \mathcal{Z}(X)$ if and only if $\mathcal{X}^{\prime}:=\mathcal{L} \backslash \mathcal{X} \in X(\mathcal{L})$. In this case, letting $x^{\prime}:=\beta^{-1}\left(\mathcal{X}^{\prime}\right) \in X$, we have $X \cong[\underline{0}, x] \times\left[\underline{0}, x^{\prime}\right]$ as inner direct product:

Let $x \in \mathcal{Z}(X)$, let $X \cong[\underline{0}, x] \times\left[\underline{0}, x^{\prime}\right]$, where $x^{\prime} \in X$, and let $\mathcal{X}^{\prime}:=\beta\left(x^{\prime}\right) \in X(\mathcal{L})$. Hence for $z \in \mathcal{L}$ we have either $z \leq x$ or $z \leq x^{\prime}$, and thus $\mathcal{L}=\{z \in \mathcal{L} ; z \leq x\} \dot{\cup}$ $\left\{z \in \mathcal{L} ; z \leq x^{\prime}\right\}=\mathcal{X} \cup \mathcal{X}^{\prime}$, hence $\mathcal{X}^{\prime}=\mathcal{L} \backslash \mathcal{X}$.

Let conversely $\mathcal{X}^{\prime}:=\mathcal{L} \backslash \mathcal{X} \in X(\mathcal{L})$ and $x^{\prime}:=\beta^{-1}\left(\mathcal{X}^{\prime}\right) \in X$. Now let $\mathcal{Y}, \mathcal{Y}^{\prime} \in$ $X(\mathcal{L})$ such that $\mathcal{Y} \subseteq \mathcal{X}$ and $\mathcal{Y}^{\prime} \subseteq \mathcal{X}^{\prime}$. Then $\mathcal{Y} \cup \mathcal{Y}^{\prime} \subseteq \mathcal{L}$ is an ideal which is bounded in $X$. Let $\mathcal{D} \subseteq \mathcal{L}$ be a dotted-line such that $\left|\overline{\mathcal{D}} \cap\left(\mathcal{Y} \cup \dot{\mathcal{Y}} \mathcal{Y}^{\prime}\right)\right| \geq 2$. Since 
$|\mathcal{D}| \geq 3$ we have $|\mathcal{D} \cap \mathcal{X}| \geq 2$ or $\left|\mathcal{D} \cap \mathcal{X}^{\prime}\right| \geq 2$, and hence either $\mathcal{D} \subseteq \mathcal{X}$ or $\mathcal{D} \subseteq \mathcal{X}^{\prime}$ Thus we have either $|\mathcal{D} \cap \mathcal{Y}| \geq 2$ or $\left|\mathcal{D} \cap \mathcal{Y}^{\prime}\right| \geq 2$, and hence either $\mathcal{D} \subseteq \mathcal{Y}$ or $\mathcal{D} \subseteq \mathcal{Y}^{\prime}$. Thus $\mathcal{Y} \cup \mathcal{Y}^{\prime} \in X(\mathcal{L})$, coinciding with the join of $\mathcal{Y}$ and $\mathcal{Y}^{\prime}$ in $X(\mathcal{L})$.

We consider the order-preserving maps $\sigma:[\underline{0}, x] \times\left[\underline{0}, x^{\prime}\right] \rightarrow X:\left[y, y^{\prime}\right] \mapsto y \vee y^{\prime}$ and $\tau: X \rightarrow[\underline{0}, x] \times\left[\underline{0}, x^{\prime}\right]: z \mapsto\left[z \wedge x, z \wedge x^{\prime}\right]$. By the above we have $\beta\left(y \vee y^{\prime}\right)=$ $\beta(y) \cup \beta\left(y^{\prime}\right)$, while for $z \in X$ we have $\beta(z \wedge x)=\beta(z) \cap \mathcal{X} \in X(\mathcal{L})$ and $\beta\left(z \wedge x^{\prime}\right)=\beta(z) \cap \mathcal{X}^{\prime} \in X(\mathcal{L})$ anyway. Thus we have $\beta(\sigma \tau(z))=\beta((z \wedge x) \vee$ $\left.\left(z \wedge x^{\prime}\right)\right)=\beta(z \wedge x) \cup \beta\left(z \wedge x^{\prime}\right)=(\beta(z) \cap \mathcal{X}) \cup\left(\beta(z) \cap \mathcal{X}^{\prime}\right)=\beta(z)$, by $(7.3)$ entailing $\sigma \tau(z)=z$. Moreover, for $\tau \sigma\left(\left[y, y^{\prime}\right]\right)=\left[\left(y \vee y^{\prime}\right) \wedge x,\left(y \vee y^{\prime}\right) \wedge x^{\prime}\right]$ we get $\beta\left(\left(y \vee y^{\prime}\right) \wedge x\right)=\beta\left(y \vee y^{\prime}\right) \cap \mathcal{X}=\left(\beta(y) \cup \beta\left(y^{\prime}\right)\right) \cap \mathcal{X}=(\beta(y) \cap \mathcal{X}) \cup\left(\beta\left(y^{\prime}\right) \cap \mathcal{X}\right)=\beta(y)$ and similarly $\beta\left(\left(y \vee y^{\prime}\right) \wedge x^{\prime}\right)=\beta\left(y \vee y^{\prime}\right) \cap \mathcal{X}^{\prime}=\left(\beta(y) \cup \beta\left(y^{\prime}\right)\right) \cap \mathcal{X}^{\prime}=(\beta(y) \cap$ $\left.\mathcal{X}^{\prime}\right) \cup\left(\beta\left(y^{\prime}\right) \cap \mathcal{X}^{\prime}\right)=\beta\left(y^{\prime}\right)$, which by $(7.3)$ entails $\tau \sigma\left(\left[y, y^{\prime}\right]\right)=\left[y, y^{\prime}\right]$. Hence $\sigma$ and $\tau$ are a pair of mutually inverse isomorphisms of lattices.

b) Let the block graph of $X$ be defined as the undirected simple graph having vertex set $\mathcal{L}$, where vertices $x, y \in \mathcal{L}$ are adjacent if and only if $x<y$ or $y<x$ or $x \vee y \in \mathcal{L}_{2}$. Vertices being in the same connected component of the block graph are called to be in the same block, giving rise to the disjoint union $\mathcal{L}=\coprod_{i=1}^{d} \mathcal{Z}_{i}$, where $d \in \mathbb{N}_{0}$ is the number of blocks occurring.

Then $\mathcal{Z}_{i} \subseteq \mathcal{L}$ is an ideal, for $i \in\{1, \ldots, d\}$, and for any dotted-line $\mathcal{D} \subseteq \mathcal{L}$ we have either $\mathcal{D} \cap \mathcal{Z}_{i}=\emptyset$ or $\mathcal{D} \subseteq \mathcal{Z}_{i}$. Since $X$ has a one element, $\mathcal{Z}_{i}$ has an upper bound in $X$, hence $\mathcal{Z}_{i} \in X(\mathcal{L})$, and we let $z_{i}:=\beta^{-1}\left(\mathcal{Z}_{i}\right) \in X$.

c) Given $x \in X$, we have $x \in \mathcal{Z}(X)$ if and only if $x=\bigvee\left\{z_{i} \in X\right.$; $i \in$ $\left.\{1, \ldots, d\}, z_{i} \leq x\right\}$. In particular, $X \cong \prod_{i=1}^{d}\left[\underline{0}, z_{i}\right]$ is the unique decomposition of $X$ into non-trivial indecomposable intervals:

Let $x \in \mathcal{Z}(X)$ and $\mathcal{X}:=\beta(x) \in X(\mathcal{L})$, and $\mathcal{X}^{\prime}:=\mathcal{L} \backslash \mathcal{X} \in X(\mathcal{L})$ and $x^{\prime}:=$ $\beta^{-1}\left(\mathcal{X}^{\prime}\right) \in X$. We consider $\mathcal{Z}_{i}$ such that $\mathcal{X} \cap \mathcal{Z}_{i} \neq \emptyset$, and show that $\mathcal{Z}_{i} \subseteq \mathcal{X}$ :

To this end let $y \in \mathcal{X} \cap \mathcal{Z}_{i}$, and $y^{\prime} \in \mathcal{Z}_{i}$ be connected to $y$ in the block graph. If $y^{\prime}<y$, then by completeness we have $y^{\prime} \in \mathcal{X}$ as well. If $y^{\prime}>y$, assume that $y^{\prime} \notin \mathcal{X}$, hence we have $y^{\prime} \in \mathcal{X}^{\prime}$, thus by completeness $y \in \mathcal{X}^{\prime}$, a contradiction; this shows $y^{\prime} \in \mathcal{X}$. If $y \vee y^{\prime} \in \mathcal{L}_{2}$, assume that $y^{\prime} \notin \mathcal{X}$, hence $y^{\prime} \in \mathcal{X}^{\prime}$; now there is a dotted-line $\mathcal{D} \subseteq \mathcal{L}$ such that $\left\{y, y^{\prime}\right\} \subseteq \mathcal{D}$, entailing $\mathcal{D} \cap \mathcal{X} \neq \emptyset$ and $\mathcal{D} \cap \mathcal{X}^{\prime} \neq \emptyset$; since $|\mathcal{D}| \geq 3$ we have $|\mathcal{D} \cap \mathcal{X}| \geq 2$ or $\left|\mathcal{D} \cap \mathcal{X}^{\prime}\right| \geq 2$, and hence by completeness either $\mathcal{D} \subseteq \mathcal{X}$ or $\mathcal{D} \subseteq \mathcal{X}^{\prime}$, a contradiction; this shows $y^{\prime} \in \mathcal{X}$.

Thus we have $\bigvee\left\{z_{i} \in X ; i \in\{1, \ldots, d\}, \mathcal{X} \cap \mathcal{Z}_{i} \neq \emptyset\right\} \leq x$ and similarly we get $\bigvee\left\{z_{j} \in X ; j \in\{1, \ldots, d\}, \mathcal{X} \cap \mathcal{Z}_{j} \neq \emptyset\right\} \leq x^{\prime}$. Since $X \cong[\underline{0}, x] \times\left[\underline{0}, x^{\prime}\right]$ and $\bigvee_{i=1}^{d} z_{i}=\underline{1}=x \vee x^{\prime}$ we conclude $x=\bigvee\left\{z_{i} \in X ; i \in\{1, \ldots, d\}, \mathcal{X} \cap \mathcal{Z}_{i} \neq \emptyset\right\}$.

Let conversely $\{1, \ldots, d\}=\mathcal{I} \cup \mathcal{J}$, and $x:=\bigvee_{i \in \mathcal{I}} z_{i}$ and $x^{\prime}:=\bigvee_{j \in \mathcal{J}} z_{j}$. Since for any dotted-line $\mathcal{D} \subseteq \mathcal{L}$ we have $\mathcal{D} \cap \mathcal{Z}_{i}=\emptyset$ or $\mathcal{D} \subseteq \mathcal{Z}_{i}$, for any $i \in\{1, \ldots, d\}$, we conclude that $\beta(x)=\bigcup_{i \in \mathcal{I}} \mathcal{Z}_{i}=\coprod_{i \in \mathcal{I}} \mathcal{Z}_{i} \in X(\mathcal{L})$ and $\beta\left(x^{\prime}\right)=\bigcup_{j \in \mathcal{J}} \mathcal{Z}_{j}=$ $\coprod_{j \in \mathcal{J}} \mathcal{Z}_{j} \in X(\mathcal{L})$. Since $\mathcal{L}=\left(\coprod_{i \in \mathcal{I}} \mathcal{Z}_{i}\right) \dot{\cup}\left(\coprod_{j \in \mathcal{J}} \mathcal{Z}_{j}\right)$ we have $x \in \mathcal{Z}(X)$, where $X \cong[\underline{0}, x] \times\left[\underline{0}, x^{\prime}\right]$. 
(7.7) Maeda's Theorem. a) Let $X$ be a modular lattice. Then we have the followung transitivity property for atoms belonging to the same connected component of the block graph: Let $x, y, z \in \mathcal{L}$ be atoms such that $x \neq y$ and $x \vee z \in \mathcal{L}_{2}$ as well as $y \vee z \in \mathcal{L}_{2}$. Then we also have $x \vee y \in \mathcal{L}_{2}$ :

We may assume that $x \vee z \neq y \vee z$ holds. Hence $(x \vee z) \wedge(y \vee z)=z$, and thus $l(x \vee y \vee z)=l(x \vee z)+l(y \vee z)-l((x \vee z) \wedge(y \vee z))=2+2-1=3$. Since $x \vee z \in \mathcal{L}_{2}$ and $y \vee z \in \mathcal{L}_{2}$ there are atoms $v, w \in X \backslash\{x, y, z\}$ such that $0 \lessdot v \lessdot x \vee z$ and $0 \lessdot w \lessdot y \vee z$. Assume that $v=w$, hence $0 \lessdot v=w \leq(x \vee z) \wedge(y \vee z)=z$, thus $v=w=z$, a contradiction. Thus we have $v \neq w$.

Let $u:=(v \vee w) \wedge(x \vee y) \in X$. Since $(v \vee w) \vee(x \vee y)=x \vee y \vee z$, we get $l(u)=l(v \vee w)+l(x \vee y)-l(x \vee y \vee z)=2+2-3=1$. Hence $0 \lessdot u \lessdot x \vee y$. Assume that $u=x$, then we have $x \leq v \vee w$, hence $v \vee w=x \vee v=x \vee z=$ $w \vee z=y \vee z$, a contradiction. Similarly, assuming $u=y$ implies $y \leq v \vee w$, thus $v \vee w=y \vee w=y \vee z=v \vee z=x \vee z$, a contradiction. Hence we have $x \neq u \neq y$, showing $x \vee y \in \mathcal{L}_{2}$.

Note that the above transitivity property does not hold for local elements in general: For the example depicted in Table 10, see (7.2), we have $b \vee d \in \mathcal{L}_{2}$ and $c \vee d \in \mathcal{L}_{2}$, but $b \vee c \notin \mathcal{L}_{2}$, actually even $c<d$.

b) We now consider complemented lattices. In this case the local elements coincide with the atoms, thus the block graph just encodes the $\mathcal{L}_{2}$ property; in view of the above observation the connected components of the block graph are complete graphs: Let $X$ be complemented; recall that $X$ has a one element $\underline{1}$. Then for atoms $x \neq y \in X$ we have $x \vee y \in \mathcal{L}_{2}$ if and only if $x$ and $y$ have a common complement in $X$ :

Let $z \in X$ be a common complement for $x$ and $y$. From $[(x \vee y) \wedge z, x \vee y] \cong$ $[z, x \vee y \vee z]=[z, \underline{1}]=[z, x \vee z] \cong[z \wedge x, x]=[\underline{0}, x]$ we get $\underline{0} \lessdot(x \vee y) \wedge z \lessdot x \vee y$. Since $x \neq(x \vee y) \wedge z \neq y$ we have $x \vee y \in \mathcal{L}_{2}$.

Let conversely $x \vee y \in \mathcal{L}_{2}$, let $z \in X \backslash\{x, y\}$ such that $\underline{0} \lessdot z \lessdot x \vee y$, and let $w \in X$ be a complement for $x \vee y$. Hence we have $x \vee(w \vee z)=w \vee(x \vee y)=1$. Moreover, we get $[x \wedge(w \vee z), x] \cong[w \vee z, x \vee(w \vee z)]=[w \vee z, w \vee(x \vee y)] \cong[z, x \vee y]$, thus $x \wedge(w \vee z) \lessdot x$ and hence $x \wedge(w \vee z)=\underline{0}$. Thus $w \vee z \in X$ is a complement for $x$. A similar argument shows that $w \vee z$ also is a complement for $y$.

c) Let still $X$ be complemented, and let $X \cong \prod_{i=1}^{d}\left[\underline{0}, z_{i}\right]$, where $d \in \mathbb{N}_{0}$, be the decomposition of $X$ into non-trivial indecomposable intervals. Then we have Maeda's Theorem, saying that for atoms $x \neq y \in X$ we have $x \vee y \in \mathcal{L}_{2}$ if and only if there is $i \in\{1, \ldots, d\}$ such that both $x \leq z_{i}$ and $y \leq z_{i}$ :

There are unique $i, j \in\{1, \ldots, d\}$ such that $x \leq z_{i}$ and $y \leq z_{j}$. If $i \neq j$, then since $\left[0, z_{i} \vee z_{j}\right] \cong\left[0, z_{i}\right] \times\left[0, z_{j}\right]$ we have $[0, x \vee y]=[0, x] \times[0, y]$, thus $x \vee y \notin \mathcal{L}_{2}$. If $i=j$, then let $\mathcal{Z}_{i}:=\beta\left(z_{i}\right) \subseteq \mathcal{L}$ be the associated block. As $\mathcal{Z}_{i}$ is a connected component of the block graph, there is a chain $x=x_{0}, x_{1}, \ldots, x_{s}=y$ in $\mathcal{L}$ such that $x_{i-1} \vee x_{i} \in \mathcal{L}_{2}$, for all $i \in\{1, \ldots, s\}$. Hence we have $x \vee y \in \mathcal{L}_{2}$ as well. $\sharp$ 
(7.8) Submodule lattices. Although these have been the original motivation for the developments in [19], we only briefly consider submodule lattices, in order to collect the facts needed later on, see (11.3):

a) Let $K$ be a field, let $\mathcal{A}$ be a finite-dimensional $K$-algebra $\mathcal{A}$, and let $V$ be a finitely generated $\mathcal{A}$-module; hence $V$ is finite-dimensional as well. Then the set $\mathcal{M}(V)$ of all $\mathcal{A}$-submodules of $V$ becomes a partially ordered set with respect to set-theoretic inclusion $\subseteq$, and thus a lattice with meet $U \cap U^{\prime}$ and join $U+U^{\prime}$.

If $X, Y, Z \leq V$ are $\mathcal{A}$-submodules such that $Z \leq X$, then we have $X \cap(Y+Z)=$ $(X \cap Y)+Z$, which holds algebraically already for $K$-vector spaces, that is $\mathcal{M}(V)$ fulfills the modular law; note that the natural isomorphism of the lattice intervals $X \cap Y \leq X$ and $Y \leq X+Y$ is induced by the isomorphism of $\mathcal{A}$ modules $X /(X \cap Y) \rightarrow(X+Y) / Y: x+(X \cap Y) \mapsto x+Y$. Moreover, $\mathcal{M}(V)$ is locally chain-finite, hence becomes a modular lattice with zero element $\{0\}$ and one element $V$; note that $\mathcal{M}(V)$ is not necessarily locally finite.

In particular, $\mathcal{M}(V)$ is graded, the length function on $\mathcal{A}$-submodules being given by composition length. Note that, by the Jordan-Hölder Theorem, whenever $\{0\}=V_{0} \lessdot V_{1} \lessdot \cdots \lessdot V_{l}=V$ is a saturated chain of $\mathcal{A}$-submodules, where $l=$ $l(V) \in \mathbb{N}_{0}$, then the multiplicity $[V: S] \in \mathbb{N}_{0}$ with which a simple $\mathcal{A}$-module $S$ occurs, up to isomorphism, as a subquoient $X_{i} / X_{i-1}$, for $i \in\{1, \ldots, l\}$, is independent of the particular saturated chain chosen; if $[V: S] \geq 1$ then $S$ is called a constituent of $V$.

b) The lattice theoretic radical of an $\mathcal{A}$-submodule $U \leq V$ coincides with its Jacobson radical $\operatorname{rad}(U)$, hence $U / \operatorname{rad}(U)$ being complemented just says that it is a semi-simple $\mathcal{A}$-module. Given a simple $\mathcal{A}$-module $S$, then $U$ is called $S$-local if $U / \operatorname{rad}(U) \cong S$. Letting $\mathcal{L}_{S}(V) \subseteq \mathcal{M}(V)$ be the set of all $S$-local $\mathcal{A}$-submodules of $V$, then $\mathcal{L}(V):=\coprod_{S \text { simple }} \mathcal{L}_{S}(V) \subseteq \mathcal{M}(V)$, the disjoint union running over the finitely many isomorphism types of simple $\mathcal{A}$-modules, is the set of all local $\mathcal{A}$-submodules of $V$; we have $\mathcal{L}(V) \neq \emptyset$ whenever $V \neq\{0\}$.

We have $\mathcal{L}_{S}(V) \neq \emptyset$ if and only if $S$ is a constituent of $V$ : If $L \in \mathcal{L}_{S}(V)$ then $L / \operatorname{rad}(L) \cong S$ shows that $S$ is a constituent of $V$. Conversely, if $U^{\prime}<U \leq V$ are $\mathcal{A}$-submodules such that $U / U^{\prime} \cong S$, then letting $L_{1}, \ldots, L_{r} \in \mathcal{L}$ such that $U=$ $\sum_{i=1}^{r} L_{i}$ is irredundant, where $r=r(U)=r(U / \operatorname{rad}(U))=l(U / \operatorname{rad}(U)) \in \mathbb{N}$, then $\operatorname{rad}(U)=\sum_{i=1}^{r} \operatorname{rad}\left(L_{i}\right)$ shows that $U / \operatorname{rad}(U) \cong \bigoplus_{i=1}^{r} L_{i} / \operatorname{rad}\left(L_{i}\right)$, hence $\operatorname{rad}(U) \leq U^{\prime}$ implies that $L_{i} / \operatorname{rad}\left(L_{i}\right) \cong S$ for some $i \in\{1, \ldots, r\}$.

In particular, we have $\left|\mathcal{L}_{S}(V)\right|=1$ if and only if $[V: S]=1$ : Assume that there are $L^{\prime}, L \in \mathcal{L}_{S}(V)$ such that $L^{\prime} \neq L$; if $L^{\prime}<L$ then from $\operatorname{rad}\left(L^{\prime}\right) \lessdot$ $L^{\prime} \leq \operatorname{rad}(L) \lessdot L$ we conclude that $[V: S] \geq 2$; if $L^{\prime} \not \leq L \not \leq L^{\prime}$ then we have $\left(L+L^{\prime}\right) / \operatorname{rad}\left(L+L^{\prime}\right) \cong L / \operatorname{rad}(L) \oplus L^{\prime} / \operatorname{rad}\left(L^{\prime}\right) \cong S \oplus S$, which again entails $[V: S] \geq 2$. Conversely, let $\mathcal{L}_{S}(V)=\{L\}$, and assume that $[V: S] \geq 2$; then there are $\mathcal{A}$-submodules $U_{1}^{\prime} \lessdot U_{1} \leq U_{2}^{\prime} \lessdot U_{2} \leq V$ such that $U_{i} / U_{i}^{\prime} \cong S$, for $i \in\{1,2\}$; letting $U_{2}=\sum_{i=1}^{r} L_{i}$, where $L_{1}, \ldots, L_{r} \in \mathcal{L}$ for some $r \in \mathbb{N}$, then there is $i \in\{1, \ldots, r\}$ such that $L_{i} \not \leq U_{2}^{\prime}$, hence $L_{i} /\left(L_{i} \cap U_{2}^{\prime}\right) \cong\left(L_{i}+U_{2}^{\prime}\right) / U_{2}^{\prime} \cong$ $U_{2} / U_{2}^{\prime} \cong S$ shows that $L_{i}=L$, thus we have $L \leq U_{2}$ but $L \not \leq U_{2}^{\prime}$; similarly we 
infer that $L \leq U_{1}$, a contradiction.

c) We proceed to describe $\mathcal{L}_{2}(V) \subseteq \mathcal{M}(V)$. In order to do so, we first we first describe the $\mathcal{A}$-submodules of $V:=S \oplus T$, where $S$ and $T$ are simple $\mathcal{A}$ modules: To this end, let $\{0\} \lessdot U \lessdot V$ be an $\mathcal{A}$-submodule, where we assume that $U \neq\{0\} \oplus T$, hence there is $\left[u, u^{\prime}\right] \in U$ such that $u \neq 0$.

If $S \neq T$, then by Wedderburn's Theorem there is $a \in \mathcal{A}$ such that $\left.a\right|_{S}=\operatorname{id}_{S}$ and $\left.a\right|_{T}=0$. Thus we have $0 \neq[u, 0] \in U$, hence from $S$ being simple we conclude that $S \oplus\{0\} \leq U$. Thus we infer that $S \oplus\{0\}$ and $\{0\} \oplus T$ are the only non-trivial proper $\mathcal{A}$-submodules of $V$.

If $S \cong T$, then we may assume that $S=T$, and let $E=E_{S}:=\operatorname{End}_{\mathcal{A}}(S)$ be the ring of $\mathcal{A}$-endomorphisms of $S$, where by Schur's Lemma $E$ is a skew field over $K$. Hence $S$ can be considered as an $E$-vector space, and by Wedderburn's Theorem again $\left.\mathcal{A}\right|_{S} \cong E^{n \times n}$, where $n:=\operatorname{dim}_{E}(S)=\frac{\operatorname{dim}_{K}(S)}{\operatorname{dim}_{K}(E)}$. If $\left\{u, u^{\prime}\right\}$ is $E$-linearly independent, then there is $a \in \mathcal{A}$ such that $u a=u$ and $u^{\prime} a=0$, hence we conclude that $0 \neq[u, 0] \in U$ and thus $U=S \oplus\{0\}$. If $\left\{u, u^{\prime}\right\}$ is $E$-linearly dependent, then $\left[u, u^{\prime}\right]=[u, u \alpha]$ for some $\alpha \in E$, and thus projecting onto the first component shows that $U=[u, u \alpha] \cdot \mathcal{A} \cong S$. Moreover, if $U=[u, u \alpha] \cdot \mathcal{A}=[u, u \beta] \cdot \mathcal{A}$, for some $\alpha \neq \beta \in E$, then $[0, u(\alpha-\beta)] \in U$, where since $\alpha-\beta \in E^{*}$ we have $u(\alpha-\beta) \neq 0$, hence $U=\{0\} \oplus T$, a contradiction. Hence the $\mathcal{A}$-submodules of $V$ different from $\{0\} \oplus S$ are in bijection with $E$ via $\alpha \mapsto[u, u \alpha] \cdot \mathcal{A}$, for some fixed $0 \neq u \in S$.

We conclude that the submodule lattice of $V \cong S \oplus T$ is indecomposable if and only if $S \cong T$. In this case, if moreover $K=\mathbb{F}_{q}$ is the finite field with $q$ elements, then $E$ is the field with $q^{\operatorname{dim}_{K}(E)}$ elements, and there are precisely $|E|+1=q^{\operatorname{dim}_{K}(E)}+1$ non-trivial proper $\mathcal{A}$-submodules of $V=S \oplus S$.

We are now prepared to describe $\mathcal{L}_{2}(V)$, where $V$ is arbitrary again: If $L, L^{\prime} \in$ $\mathcal{L}(V)$ such that $L \not \leq L^{\prime} \not \leq L$, then letting $Z:=L+L^{\prime} \leq V$ we have $Z / \operatorname{rad}(Z) \cong$ $L / \operatorname{rad}(L) \oplus L^{\prime} / \operatorname{rad}\left(L^{\prime}\right)$, hence we have $Z \in \mathcal{L}_{2}(V)$ if and only if $L / \operatorname{rad}(L) \cong$ $L^{\prime} / \operatorname{rad}\left(L^{\prime}\right)$, that is there is a simple $\mathcal{A}$-module $S$ such that both $L, L^{\prime} \in \mathcal{L}_{S}(V)$; in this case we may let $\mathcal{I}_{Z}:=E_{S} \cup \dot{\cup}\{\infty\}$. In conclusion, accompanying the disjoint decomposition of $\mathcal{L}(V)$, we have a finite disjoint union $\mathcal{L}_{2}(V):=$ $\amalg_{S \text { simple }} \mathcal{L}_{2, S}(V) \subseteq \mathcal{M}(V)$, where in turn we have $\mathcal{L}_{2, S}(V)=\left\{L+L^{\prime} \in\right.$ $\left.\mathcal{M}(V) ; L, L^{\prime} \leq \mathcal{L}_{S}(V), L \not \leq L^{\prime} \not \leq L\right\}$.

In particular, we have $\mathcal{L}_{2, S}(V)=\emptyset$ if and only if $\mathcal{L}_{S}(V)=\emptyset$ or $\mathcal{L}_{S}(V)$ is a chain. Moreover, dotted-lines can be computed by considering each simple $\mathcal{A}$-module in turn, and all dotted-lines belonging to one and the same simple $\mathcal{A}$-module have the same cardinality. Finally, letting $\mathcal{S}(V)$ be the set of simple $\mathcal{A}$-modules, up to isomorphism, which occur as constituents of $V$, and letting $\mathcal{L}(V)=\coprod_{i=1}^{d} \mathcal{Z}_{i}$ be the block decomposition, where $d \in \mathbb{N}_{0}$, from the definition of the block graph we infer that there is a corresponding partition $\mathcal{S}(V)=\coprod_{i=1}^{d} \mathcal{S}_{i}$.

d) In particular, by Birkhoff's Representation Theorem, $\mathcal{M}(V)$ is a distributive lattice if and only if $\mathcal{L}_{2}(V)=\emptyset$, that is $\mathcal{L}_{2, S}(V)=\emptyset$ for all simple $\mathcal{A}$-modules 
$S$, which in turn holds if and only if $\mathcal{L}_{S}(V)$ is a chain for all constituents $S$ of $V$. In particular, this is the case if $[V: S] \leq 1$ for all simple $\mathcal{A}$-modules $S$, that is $V$ is multiplicity-free. Recall that if $\mathcal{M}(V)$ is distributive then it is finite, being in natural bijection with the partially ordered set of ideals of $\mathcal{L}(V)$.

If $\mathcal{M}(V)$ is distributive, then the indecomposable direct summands of the $\mathcal{A}$ module $V$ are precisely given by the block graph; note that since $\mathcal{L}_{2}(V)=$ $\emptyset$, the latter just encodes the mutual inclusion of local $\mathcal{A}$-submodules: Let $\mathcal{L}(V)=\coprod_{i=1}^{d} \mathcal{Z}_{i}$ be the block decomposition, where $d \in \mathbb{N}_{0}$. Then letting $Z_{i}:=\beta^{-1}\left(\mathcal{Z}_{i}\right)=\mathcal{Z}_{i} \cdot \mathcal{A} \leq V$ be the $\mathcal{A}$-submodule generated by $\mathcal{Z}_{i}$, where $\beta$ is as in (7.3), we have the lattice block decomposition $V=\bigoplus_{i=1}^{d} Z_{i}$ as $\mathcal{A}$-modules. Hence it remains to be shown that the $Z_{i}$ are indecomposable:

Assume that $Z_{i}=Z_{i}^{\prime} \oplus Z_{i}^{\prime \prime}$ as $\mathcal{A}$-modules, where $Z_{i}^{\prime} \neq\{0\} \neq Z_{i}^{\prime \prime}$. Then, assume there is a $S \in \mathcal{S}_{i}$ being a constituent of both $Z_{i}^{\prime}$ and $Z_{i}^{\prime \prime}$; then there are $S$ local submodules $L^{\prime} \leq Z_{i}^{\prime}$ and $L^{\prime \prime} \leq Z_{i}^{\prime \prime}$, which hence fulfill $L^{\prime} \not L^{\prime \prime} \not L^{\prime}$, implying that $\mathcal{L}_{2, S}(V) \neq \emptyset$, a contradiction. Thus we have a corresponding partition $\mathcal{S}_{i}=\mathcal{S}_{i}^{\prime} \dot{\cup} \mathcal{S}_{i}^{\prime \prime}$, where $\mathcal{S}_{i}^{\prime} \neq \emptyset \neq \mathcal{S}_{i}^{\prime \prime}$, entailing a non-trivial partition $\coprod_{S \in \mathcal{S}_{i}} \mathcal{L}_{S}(V)=\coprod_{S \in \mathcal{S}_{i}^{\prime}} \mathcal{L}_{S}(V) \dot{\cup} \coprod_{S \in \mathcal{S}_{i}^{\prime \prime}} \mathcal{L}_{S}(V)$, where there are no inclusions between the local $\mathcal{A}$-submodules in the left hand and right hand parts. From this we conclude that the latter are contained in distinct connected components of the block graph, a contradiction.

\section{Incidence algebras}

\section{Incidence algebras}

(8.1) The incidence algebra. a) Let $X$ be a locally finite partially ordered set, let $R \neq\{0\}$ be a commutative ring, and let $\mathcal{A}_{R}(X):=\{f \in \operatorname{Maps}(X \times$ $X, R) ; f(x, y)=0$ if $x \not \leq y\}$. Then $\mathcal{A}_{R}(X)$ is an $R$-module respect to pointwise addition $(f+g)(x, y):=f(x, y)+g(x, y)$ and pointwise scalar multiplication $(c f)(x, y):=c \cdot f(x, y)$, for all $f, g \in \mathcal{A}_{R}(X)$ and $x, y \in X$ and $c \in R$.

Convolutional multiplication on $\mathcal{A}_{R}(X)$ is well-defined by letting $(f g)(x, y):=$ $\sum_{z \in X} f(x, z) g(z, y)$, for all $f, g \in \mathcal{A}_{R}(X)$ and $x, y \in X$ : If $x \not \leq y$ then all summands vanish, hence $(f g)(x, y)=0$, while if $x \leq y$ we get $(f g)(x, y)=$ $\sum_{x \leq z \leq y} f(x, z) g(z, y)$, which by local finiteness is a finite sum. Then we have $(f g) h=f(g h):[x, y] \mapsto \sum_{z, z^{\prime} \in X} f(x, z) g\left(z, z^{\prime}\right) h\left(z^{\prime}, y\right)$, for all $f, g, h \in \mathcal{A}_{R}(X)$, that is associativity holds. Moreover, for the map $\delta: X \times X \rightarrow R:[x, y] \mapsto \delta_{x, y}$, where $\delta_{x, y}$ is the Kronecker symbol, we have $(f \delta)(x, y)=\sum_{z \in X} f(x, z) \delta(z, y)=$ $f(x, y)$ and $(\delta f)(x, y)=\sum_{z \in X} \delta(x, z) f(z, y)=f(x, y)$, for all $x, y \in X$, hence $\delta \in \mathcal{A}_{R}(X)$ is a neutral element with respect to convolutional multiplication. Since we have distributivity, and $c(f g)=(c f) g=f(c g) \in \mathcal{A}_{R}(X)$, for all $c \in R$, we conclude that $\mathcal{A}_{R}(X)$ is a non-commutative $R$-algebra, called the incidence algebra associated with the partially ordered set $X$. 
b) Let $f \in \mathcal{A}_{R}(X)$. We show that $f$ is right invertible, if and only if $f$ is left invertible, which holds if and only if $f(x, x) \in R^{*}$ for all $x \in X$ :

The map $g \in \mathcal{A}_{R}(X)$ is a right inverse of $f$ if and only if $\delta_{x, y}=(f g)(x, y)=$ $\sum_{z \in X} f(x, z) g(z, y)$, for all $x, y \in X$. Hence, for $x=y$, from the existence of $g$ we infer $f(x, x) \in R^{*}$. Conversely, if $f(x, x) \in R^{*}$ for all $x \in X$, we define $g \in \mathcal{A}_{R}(X)$ using local finiteness as follows: We let $g(x, x):=f(x, x)^{-1} \in R$ for all $x \in X$, and for $x<y \in X$, by induction on $l(x, y) \in \mathbb{N}_{0}$ assuming that $g(z, y)$ has already been found for all $x<z \leq y \in X$, we let $g(x, y)=$ $-f(x, x)^{-1} \cdot \sum_{x<z \leq y} f(x, z) g(z, y) \in R$. Then we have $\sum_{x \leq z \leq y} f(x, z) g(z, y)=$ $f(x, x) g(x, y)+\sum_{x<z \leq y} f(x, z) g(z, y)=0$.

Similarly, the map $g \in \mathcal{A}_{R}(X)$ is a left inverse of $f$ if and only if $\delta_{x, y}=$ $(g f)(x, y)=\sum_{z \in X} g(x, z) f(z, y)$, for all $x, y \in X$. Hence, for $x=y$, from the existence of $g$ we infer $f(x, x) \in R^{*}$. Conversely, if $f(x, x) \in R^{*}$ for all $x \in X$, we define $g \in \mathcal{A}_{R}(X)$ using local finiteness as follows: We let $g(x, x):=f(x, x)^{-1} \in R$ for all $x \in X$, and for $x<y \in X$, by induction on $l(x, y) \in \mathbb{N}_{0}$ assuming that $g(x, z)$ has already been found for all $x \leq z<y \in X$, we let $g(x, y)=-f(y, y)^{-1} \cdot \sum_{x<z<y} g(x, z) f(z, y) \in R$. Then we have $\sum_{x \leq z \leq y} g(x, z) f(z, y)=g(x, y) f(y, y)+\sum_{x \leq z<y} g(x, z) f(z, y)=0$. $\sharp$

Note that, in this case, right and left inverses coincide and are uniquely defined, giving rise to the unit group $\mathcal{A}_{R}^{*}(X)$ of $\mathcal{A}_{R}(X)$. Note that for $f \in \mathcal{A}_{R}^{*}(X)$ the value $f^{-1}(x, y) \in R$, for $x \leq y \in X$, only depends on the interval $[x, y] \subseteq X$.

c) Let $\operatorname{rad}(R)$ denote the Jacobson radical of $R$. We show that $\operatorname{rad}\left(\mathcal{A}_{R}(X)\right)=$ $\mathcal{J}_{R}(X):=\left\{f \in \mathcal{A}_{R}(X) ; f(x, x) \in \operatorname{rad}(R)\right.$ for all $\left.x \in X\right\}$ :

Let $f \in \mathcal{J}_{R}(X)$. Then for any $x \in X$ and any $a \in R$ we have $1+a \cdot f(x, x) \in R^{*}$. Hence for any $g \in \mathcal{A}_{R}(X)$ we have $(\delta+f g)(x, x)=1+f(x, x) g(x, x) \in R^{*}$, for all $x \in X$, thus $\delta+f g \in \mathcal{A}_{R}^{*}(X)$, implying that $f \in \operatorname{rad}\left(\mathcal{A}_{R}(X)\right)$. Conversely, if $f \in \mathcal{A}_{R}(X)$ such that $f(x, x) \notin \operatorname{rad}(R)$ for some $x \in X$, then there is $a \in R$ such that $1+a \cdot f(x, x) \notin R^{*}$. Hence picking $g \in \mathcal{A}_{R}(X)$ such that $g(x, x)=a$, we infer $(\delta+f g)(x, x) \notin R^{*}$, thus $\delta+f g \notin \mathcal{A}_{R}^{*}(X)$, hence $f \notin \operatorname{rad}\left(\mathcal{A}_{R}(X)\right)$. $\sharp$

Hence we have $\mathcal{A}_{R}(X) / \mathcal{J}_{R}(X) \cong \prod_{x \in X} R / \operatorname{rad}(R)$. Moreover, letting $\mathcal{U}_{R}(X):=$ $\left\{f \in \mathcal{A}_{R}(X) ; f(x, x)=0\right.$ for all $\left.x \in X\right\} \subseteq \mathcal{J}_{n}(R)$, then from $(f g)(x, x)=$ $f(x, x) g(x, x)$, for all $f, g \in \mathcal{A}_{R}(X)$ and $x \in X$, we infer that $\mathcal{U}_{R}(X) \unlhd \mathcal{A}_{R}(X)$ is an ideal, and we have $\mathcal{A}_{R}(X) / \mathcal{U}_{R}(X) \cong \prod_{x \in X} R$.

(8.2) Zeta and Möbius functions. a) Let $X$ be a locally finite partially ordered set, and let $\zeta \in \mathcal{A}(X)=\mathcal{A}_{\mathbb{Z}}(X)$ be the zeta function of $X$, that is the indicator function of the partial order, given by $\zeta(x, y)=1$ whenever $x \leq y \in X$, and $\zeta(x, y)=0$ whenever $x \not \leq y \in X$; in particular, we have $\zeta \in \mathcal{A}^{*}(X)$. Note that restricting $\zeta$ to an interval in $X$ just yields the zeta function of the interval. The zeta function is related to chains:

i) Induction on $k \in \mathbb{N}$ yields $\zeta^{k}(x, y)=\sum_{x=z_{0} \leq z_{1} \leq \cdots \leq z_{k}=y} \prod_{i=1}^{k} \zeta\left(z_{i-1}, z_{i}\right)=$ $\sum_{x=z_{0} \leq z_{1} \leq \cdots \leq z_{k}=y} 1$, for $x \leq y \in X$. Thus for $k \in \mathbb{N}_{0}$ we infer that $\zeta^{k}(x, y) \in \mathbb{N}_{0}$ 
is the number of multichains of length $k$, that is chains with repeated entries, between $x$ and $y$; in particular, $\zeta^{2}(x, y)=|[x, y]|$ for all $x \leq y \in X$.

ii) We have $(\zeta-\delta)(x, x)=0$, and $(\zeta-\delta)(x, y)=1$ for all $x<y \in X$; in particular, we have $\zeta-\delta \in \mathcal{J}(X)=\mathcal{J}_{\mathbb{Z}}(X)$. For this function, induction on $k \in \mathbb{N}$ yields $(\zeta-\delta)^{k}(x, y)=\sum_{x=z_{0} \leq z_{1} \leq \cdots \leq z_{k}=y} \prod_{i=1}^{k}(\zeta-\delta)\left(z_{i-1}, z_{i}\right)=$ $\sum_{x=z_{0}<z_{1}<\cdots<z_{k}=y} 1 \in \mathbb{N}_{0}$, for all $x \leq y \in X$, saying that for $k \in \mathbb{N}_{0}$ there are precisely $(\zeta-\delta)^{k}(x, y) \in \mathbb{N}_{0}$ chains of length $k$ between $x$ and $y$. In particular, we have $(\zeta-\delta)^{k}(x, y)=0$ if and only if $k \geq l+1$, where $l:=l(x, y) \in \mathbb{N}_{0}$, saying that $\zeta-\delta$ is locally nilpotent, that is nilpotent on any interval $[x, y]$.

iii) We have $(2 \delta-\zeta)(x, x)=1$, and $(2 \delta-\zeta)(x, y)=-1$ for all $x<y \in X$, hence again $2 \delta-\zeta \in \mathcal{A}^{*}(X)$. Then we have $(2 \delta-\zeta)^{-1}=\sum_{i \geq 0}(\zeta-\delta)^{i} \in \mathcal{A}(X)$, which is indeed well-defined inasmuch it says that $(2 \delta-\zeta)^{-1}(x, y) \in \mathbb{N}_{0}$ coincides with the number of all chains between $x, y \in X$ : We may assume that $x \leq y$, and letting $l:=l(x, y) \in \mathbb{N}_{0}$ we have $\left.(\zeta-\delta)^{l+1}\right|_{[x, y]}=0$, hence upon restriction to $[x, y]$ we have $(2 \delta-\zeta) \cdot \sum_{i=0}^{l}(\zeta-\delta)^{i}=(\delta-(\zeta-\delta)) \cdot \sum_{i=0}^{l}(\zeta-\delta)^{i}=\delta-(\zeta-\delta)^{l+1}=\delta$, and thus $(2 \delta-\zeta)^{-1}(x, y)=\sum_{i=0}^{l}(\zeta-\delta)^{i}(x, y)$.

b) The zeta function $\zeta \in \mathcal{A}(X)$ being invertible, we let $\mu:=\zeta^{-1} \in \mathcal{A}(X)$ be the Möbius function of $X$. More precisely, we have $\mu(x, x)=1$ for all $x \in X$, and for $x<y \in X$ using the right inversion formula we get $\mu(x, y)=$ $-\sum_{x<z \leq y} \mu(z, y)$, or equivalently $\sum_{x \leq z \leq y} \mu(z, y)=0$, while the left inversion formula yields $\mu(x, y)=-\sum_{x \leq z<y} \mu(x, z)$, or equivalently $\sum_{x \leq z \leq y} \mu(x, z)=0$; in particular, $\mu(x, y) \in \mathbb{Z}$ can be computed by induction on $l(x, y) \in \mathbb{N}_{0}$.

Letting $x \leq y \in X$ and $l:=l(x, y) \in \mathbb{N}_{0}$ we have $\left.(\zeta-\delta)^{l+1}\right|_{[x, y]}=0$, hence upon restriction to $[x, y]$ we have $\zeta \cdot \sum_{i=0}^{l}(\delta-\zeta)^{i}=(\delta-(\delta-\zeta)) \cdot \sum_{i=0}^{l}(\delta-\zeta)^{i}=$ $\delta-(\delta-\zeta)^{l+1}=\delta$. Hence we have Hall's Theorem [1939] $\mu(x, y)=\zeta^{-1}(x, y)=$ $\sum_{i=0}^{l}(\delta-\zeta)^{i}(x, y)=\sum_{i=0}^{l}(-1)^{i} \cdot(\zeta-\delta)^{i}(x, y)=\sum_{i>0}(-1)^{i} \cdot(\zeta-\delta)^{i}(x, y)$ expressing $\mu(x, y)$ as the alternating sum over the number of chains of lengths $i \geq 0$ between $x, y \in X$, in other words $\mu=\sum_{i \geq 0}(-1)^{i} \cdot(\zeta-\delta)^{i} \in \mathcal{A}(X)$.

c) Given $f \in \mathcal{A}(X)$, multiplication with $\zeta$ yields $f^{+}:=f \zeta \in \mathcal{A}(X)$ and $f_{+}:=$ $\zeta f \in \mathcal{A}(X)$ given by $\left(f^{+}\right)(x, y)=\sum_{x \leq z \leq y} f(x, z) \zeta(z, y)=\sum_{x \leq z \leq y} f(x, z)$ and $\left(f_{+}\right)(x, y)=\sum_{x \leq z \leq y} \zeta(x, z) f(z, y)=\sum_{x \leq z \leq y} f(z, y)$, for all $x \leq y \in X$. Then we have the Möbius inversion formulae [Rota, 1964] $f^{+} \mu=f \in \mathcal{A}(X)$, that is $f(x, y)=\sum_{x \leq z \leq y} f^{+}(x, z) \mu(z, y)$ for all $x \leq y \in X$, and $\mu f_{+}=f \in \mathcal{A}(X)$, that is $f(x, y)=\sum_{x \leq z \leq y} \mu(x, z) f_{+}(z, y)$ for all $x \leq y \in X$.

(8.3) Modules for the incidence algebra. Let $X$ be a locally finite partially ordered set, let $R \neq\{0\}$ be a commutative ring. We are looking for $\mathcal{A}_{R}(X)$-modules, apart from those coming from the quotients $\mathcal{A}_{R}(X) / \mathcal{J}_{R}(X)$ and $\mathcal{A}_{R}(X) / \mathcal{U}_{R}(X)$ considered earlier. We mainly restrict ourselves to right $\mathcal{A}_{R}(X)$-modules, which we usually just call $\mathcal{A}_{R}(X)$-modules, and where for left $\mathcal{A}_{R}(X)$-modules we typically have the 'dual' picture. The first natural choice is to look for right and left ideals in $\mathcal{A}_{R}(X)$ : 
a) For a subset $Y \subseteq X$ let $\mathcal{I}_{R}(Y, X):=\left\{f \in \mathcal{A}_{R}(X) ; f(x, ?) \neq 0\right.$ only if $\left.x \in Y\right\}$. Then $\mathcal{I}_{R}(Y, X) \leq \mathcal{A}_{R}(X)$ is a right ideal: For $f \in \mathcal{I}_{R}(Y, X)$ and $g \in \mathcal{A}_{R}(X)$, from $(f g)(x, y)=\sum_{x \leq z \leq y} f(x, z) g(z, y)$, for all $x \leq y \in X$, we get $(f g)(x, y)=$ 0 if $x \notin Y$, thus $f g \in \mathcal{I}_{R}(Y, X)$. We have $\mathcal{A}_{R}(X) \cong \mathcal{I}_{R}(Y, X) \oplus \mathcal{I}_{R}(X \backslash$ $Y, X)$, thus $\mathcal{I}_{R}(Y, X)$ is a projective $\mathcal{A}_{R}(X)$-module. Its Jacobson radical is $\operatorname{rad}\left(\mathcal{I}_{R}(Y, X)\right)=\mathcal{I}_{R}(Y, X) \cap \mathcal{J}_{R}(X)=\left\{f \in \mathcal{I}_{R}(Y, X) ; f(x, x) \in \operatorname{rad}(R)\right.$ for $x \in$ $Y\}$, hence we have $\mathcal{I}_{R}(Y, X) / \operatorname{rad}\left(\mathcal{I}_{R}(Y, X)\right) \cong \prod_{x \in Y} R / \operatorname{rad}(R)$. Moreover, we have $\mathcal{I}_{R}(Y, X) \cong \prod_{x \in Y} \mathcal{I}_{R}(\{y\}, X)$ as $\mathcal{A}_{R}(X)$-modules.

Similarly, let $\mathcal{I}_{R}(X, Y):=\left\{f \in \mathcal{A}_{R}(X) ; f(?, y) \neq 0\right.$ only if $\left.y \in Y\right\}$. Then $\mathcal{I}_{R}(X, Y) \leq \mathcal{A}_{R}(X)$ is a left ideal: For $f \in \mathcal{I}_{R}(X, Y)$ and $g \in \mathcal{A}_{R}(X)$, from $(g f)(x, y)=\sum_{x \leq z \leq y} g(x, z) f(z, y)$, for all $x \leq y \in X$, we get $(g f)(x, y)=0$

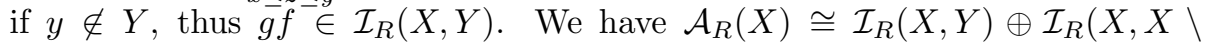
$Y)$, thus $\mathcal{I}_{R}(X, Y)$ is a projective left $\mathcal{A}_{R}(X)$-module. Its Jacobson radical is $\operatorname{rad}\left(\mathcal{I}_{R}(X, Y)\right)=\mathcal{I}_{R}(X, Y) \cap \mathcal{J}_{R}(X)=\left\{f \in \mathcal{I}_{R}(X, Y) ; f(x, x) \in \operatorname{rad}(R)\right.$ for $x \in$ $Y\}$, hence we have $\mathcal{I}_{R}(X, Y) / \operatorname{rad}\left(\mathcal{I}_{R}(X, Y)\right) \cong \prod_{x \in Y} R / \operatorname{rad}(R)$. Moreover, we have $\mathcal{I}_{R}(X, Y) \cong \prod_{x \in Y} \mathcal{I}_{R}(X,\{x\})$ as left $\mathcal{A}_{R}(X)$-modules.

b) For a coideal $Y \subseteq X$ the left ideal $\mathcal{I}_{R}(X, Y)$ also is a right ideal: For $f \in \mathcal{I}_{R}(X, Y)$ and $g \in \mathcal{A}_{R}(X)$, we have $(f g)(x, y)=\sum_{x \leq z \leq y} f(x, z) g(z, y)$, for all $x \leq y \in X$, hence, since for $y \notin Y$ we also have $z \notin \bar{Y}$ for all $z \leq y$, we conclude $(f g)(x, y)=0$ if $y \notin Y$, thus $f g \in \mathcal{I}_{R}(X, Y)$.

Combining these constructions, for any subset $Z \subseteq X$ and any coideal $Y \subseteq X$ we get the right ideal $\mathcal{I}_{R}(Z, Y):=\mathcal{I}_{R}(Z, X) \cap \mathcal{I}_{R}(X, Y) \leq \mathcal{A}_{R}(X)$. Moreover, we have $\mathcal{I}_{R}(Z, Y) \cong \prod_{x \in Z} \mathcal{I}_{R}(\{x\}, Y)$ as well as $\mathcal{I}_{R}(Z, X) / \mathcal{I}_{R}(Z, Y) \cong$ $\prod_{x \in Z} \mathcal{I}_{R}(\{x\}, X) / \mathcal{I}_{R}(\{x\}, Y)$ as $\mathcal{A}_{R}(X)$-modules. In this sense, coideals naturally provide submodules and quotient modules of the projective $\mathcal{A}_{R}(X)$ modules $\mathcal{I}_{R}(Z, X)$. Note that for any ideal $Y \subseteq X$ the complement $X \backslash Y \subseteq X$ is a coideal, so that this essentially also applies to ideals.

(8.4) The Möbius space. Let $X$ be a locally finite partially ordered set, let $R \neq\{0\}$ be a commutative ring, and let $\mathcal{F}_{R}(X):=\operatorname{Maps}(X, R)$ be the associated Möbius space, which is an $R$-module with respect to pointwise addition and scalar multiplication.

a) Let $X$ additionally have a zero element $\underline{0}$. Then we get an injective $R$-linear $\operatorname{map} \mathcal{F}_{R}(X) \rightarrow \mathcal{A}_{R}(X): f \mapsto f^{\circ}$, where $f^{\circ}(x, y):=\delta(\underline{0}, x) f(y)$, for all $x, y \in X$. Its image $\mathcal{F}_{R}^{\circ}(X):=\left\{f \in \mathcal{A}_{R}(X) ; f(x, ?) \neq 0\right.$ only if $\left.x=\underline{0}\right\}=\mathcal{I}_{R}(\{\underline{0}\}, X) \leq$ $\mathcal{A}_{R}(X)$ is a right ideal and a projective $\mathcal{A}_{R}(X)$-module, whose Jacobson radical is given as $\operatorname{rad}\left(\mathcal{F}_{R}^{\circ}(X)\right)=\left\{f \in \mathcal{F}_{R}^{\circ}(X) ; f(\underline{0}, \underline{0}) \in \operatorname{rad}(R)\right\}$.

We have $\left(f^{\circ} g\right)(\underline{0}, y)=\sum_{z \leq y} f(z) g(z, y)$, for $f \in \mathcal{F}_{R}(X)$ and $g \in \mathcal{A}_{R}(X)$, and all $y \in X$. This shows that $\mathcal{F}_{R}(X)$ becomes an $\mathcal{A}_{R}(X)$-module by letting $f * g \in \mathcal{F}_{R}(X)$ be defined as $(f * g)(x):=\sum_{z<x} f(z) g(z, x)$, for $x \in X$. Then $\mathcal{F}_{R}(X) \rightarrow \mathcal{A}_{R}(X): f \mapsto f^{\circ}$ is an $\mathcal{A}_{R}(X)$-module isomorphism, that is $(f * g)^{\circ}=$ $f^{\circ} g \in \mathcal{A}_{R}(X)$. In particular, $\mathcal{F}_{R}(X)$ is a projective $\mathcal{A}_{R}(X)$-module, such that $\operatorname{rad}\left(\mathcal{F}_{R}(X)\right)=\left\{f \in \mathcal{F}_{R}(X) ; f(\underline{0}) \in \operatorname{rad}(R)\right\}$. 
b) If $X$ instead has a one element $\underline{1}$, we get an injective $R$-linear map $\mathcal{F}_{R}(X) \rightarrow$ $\mathcal{A}_{R}(X): f \mapsto f^{\bullet}$, where now $f^{\bullet}(x, y):=\delta(y, \underline{1}) f(x)$, for all $x, y \in X$. Its image $\mathcal{F}_{R}^{\bullet}(X)=\left\{f \in \mathcal{A}_{R}(X) ; f(?, y) \neq 0\right.$ only if $\left.y=\underline{1}\right\}=\mathcal{I}_{R}(X,\{\underline{1}\}) \leq \mathcal{A}_{R}(X)$ is a left ideal and a projective left $\mathcal{A}_{R}(X)$-module, whose Jacobson radical is given as $\operatorname{rad}\left(\mathcal{F}_{R}^{\bullet}(X)\right)=\mathcal{F}_{R}^{\bullet}(X) \cap \mathcal{J}_{R}(X)=\left\{f \in \mathcal{F}_{R}^{\bullet}(X) ; f(\underline{1}, \underline{1}) \in \operatorname{rad}(R)\right\}$.

We have $\left(g f^{\bullet}\right)(x, \underline{1})=\sum_{z>x} g(x, z) f(z)$, for $f \in \mathcal{F}_{R}(X)$ and $g \in \mathcal{A}_{R}(X)$, and all $x \in X$. This shows that $\mathcal{F}_{R}(X)$ also becomes a left $\mathcal{A}_{R}(X)$-module by letting $g * f \in \mathcal{F}_{R}(X)$ be defined as $(g * f)(x):=\sum_{z>x} g(x, z) f(z)$, for $x \in X$. Then $\mathcal{F}_{R}(X) \rightarrow \mathcal{A}_{R}(X): f \mapsto f^{\bullet}$ is an $\mathcal{A}_{R}(X)$-module isomorphism, that is $(g * f)^{\bullet}=g f^{\bullet} \in \mathcal{A}_{R}(X)$. In particular, $\mathcal{F}_{R}(X)$ is a projective left $\mathcal{A}_{R}(X)$ module, such that $\operatorname{rad}\left(\mathcal{F}_{R}(X)\right)=\left\{f \in \mathcal{F}_{R}(X) ; f(\underline{1}) \in \operatorname{rad}(R)\right\}$.

But note that $\mathcal{F}_{R}(X)$ in general does not become an $\mathcal{A}_{R}(X)-\mathcal{A}_{R}(X)$-bimodule.

c) In terms of $\mathcal{F}(X)=\mathcal{F}_{\mathbb{Z}}(X)$ Möbius inversion reads as follows:

If $X$ has a zero element, using the $\mathcal{A}(X)$-module structure on $\mathcal{F}(X)$, we let $f^{+}:=f * \zeta \in \mathcal{F}(X)$, hence $f^{+}(x)=\sum_{z \leq x} f(z)$, for all $x \in X$; note that $\left(f^{+}\right)^{\circ}=\left(f^{\circ}\right)^{+} \in \mathcal{A}(X)$. Thus Möbius inversion yields $f^{+} * \mu=(f * \zeta) * \mu=$ $f *(\zeta \mu)=f * \delta=f \in \mathcal{F}(X)$, hence $f(x)=\sum_{z \leq x} f^{+}(z) \mu(z, x)$, for all $x \in X$.

If $X$ has a one element, using the left $\mathcal{A}(X)$-module structure on $\mathcal{F}(X)$, we let $f_{+}:=\zeta * f \in \mathcal{F}(X)$, hence $f_{+}(x)=\sum_{z>x} f(z)$, for all $x \in X$; note that $\left(f_{+}\right)^{\bullet}=\left(f^{\bullet}\right)_{+} \in \mathcal{A}(X)$. Thus Möbius inversion yields $\mu * f_{+}=\mu *(\zeta * f)=$ $(\mu \zeta) * f=\delta * f=f \in \mathcal{F}(X)$, hence $f(x)=\sum_{z \geq x} \mu(x, z) f_{+}(z)$, for all $x \in X$.

(8.5) Finite partially ordered sets. a) We consider the case of $X$ being finite. In order to do so, let $X=\left\{x_{1}, \ldots, x_{n}\right\}$, where $n:=|X| \in \mathbb{N}$, where we assume that $x_{i} \leq x_{j}$ only if $i \leq j \in\{1, \ldots, n\}$; note that this amounts to refining the given partial order on $X$ to a total order. This lends to the following description of $\mathcal{A}_{R}(X)$, where $R \neq\{0\}$ is a commutative ring:

For $n \in \mathbb{N}$ let $\mathcal{T}_{n}(R):=\left\{A=\left[a_{i j}\right]_{i j} \in R^{n \times n} ; a_{i j}=0\right.$ for all $\left.1 \leq j<i \leq n\right\}$ be the set of all upper triangular $(n \times n)$-matrices over $R$, and let $\mathcal{U}_{n}(R):=\{A \in$ $\mathcal{T}_{n}(R) ; a_{i i}=0$ for all $\left.i\right\} \unlhd \mathcal{T}_{n}(R)$ be the ideal consisting of all strictly upper triangular matrices. Then the injective $R$-linear map $\mathcal{A}_{R}(X) \rightarrow \mathcal{T}_{n}(R): f \mapsto$ $\left[f\left(x_{i}, x_{j}\right)\right]_{i j}$ translates convolutional multiplication into matrix multiplication, hence is a homomorphism of $R$-algebras. Thus we may and will view $\mathcal{A}_{R}(X)$ as an $R$-subalgebra of $\mathcal{T}_{n}(R)$.

b) In particular, we have $\mathcal{A}_{R}(X)=\mathcal{T}_{n}(R)$ if and only if $X$ is totally ordered. Thus $\mathcal{T}_{n}(R)$ can be considered as an incidence algebra, too. Doing so, we infer that $\mathcal{T}_{n}{ }^{*}(R)=\left\{A \in \mathcal{T}_{n}(R) ; a_{i i} \in R^{*}\right.$ for all $\left.i\right\}$, and that $\operatorname{rad}\left(\mathcal{T}_{n}(R)\right)=\mathcal{J}_{n}(R):=$ $\left\{A \in \mathcal{T}_{n}(R) ; a_{i i} \in \operatorname{rad}(R)\right.$ for all $\left.i\right\}$. Moreover, we have $\mathcal{A}_{R}^{*}(X)=\mathcal{A}_{R}(X) \cap$ $\mathcal{T}_{n}^{*}(R)$, and $\mathcal{J}_{R}(X)=\mathcal{A}_{R}(X) \cap \mathcal{J}_{n}(R)$, and $\mathcal{U}_{R}(X):=\mathcal{A}_{R}(X) \cap \mathcal{U}_{n}(R)$.

We show that $\mathcal{U}_{R}(X) \unlhd \mathcal{A}_{R}(X)$ actually is a nilpotent ideal:

For $i, j \in\{1, \ldots, n\}$ let $E_{i j} \in R^{n \times n}$ be the associated matrix unit, that is having $[k, l]$-entry $\delta_{i k} \delta_{j l} \in R$; recall the multiplication rule $E_{i j} E_{k l}=\delta_{j k} \cdot E_{i l}$. 
Then we have $\mathcal{A}_{R}(X)=\left\langle E_{x y} ; x, y \in X, x \leq y\right\rangle_{R}$ and $\mathcal{U}_{R}(X)=\left\langle E_{x y} ; x, y \in\right.$ $X, x<y\rangle_{R}$, where the given generating sets are even $R$-bases. For $k \in \mathbb{N}_{0}$ we have $\mathcal{U}_{R}(X)^{k} \leq\left\langle E_{i j} ; 1 \leq i \leq j-k \leq n\right\rangle_{R}=\left\{A \in \mathcal{T}_{n}(R) ; a_{i j}=0\right.$ for all $i>$ $j-k\}$, and we have $E_{x y} \in \mathcal{U}_{R}(X)^{k}$, where $x \leq y$, if and only if there is a chain of length $k$ between $x$ and $y$, that is $l(x, y) \geq k$. Thus we have $\mathcal{U}_{R}(X)^{k}=\{0\}$ if and only if $k \geq l(X)+1$.

As for the Möbius space, writing $f \in \mathcal{F}_{R}(X)$ as $\left[f\left(x_{1}\right), \ldots, f\left(x_{n}\right)\right] \in R^{n}$, the embedding $\mathcal{F}_{R}^{\circ}(X) \subseteq \mathcal{A}_{R}(X) \subseteq \mathcal{T}_{n}(R)$ is given by associating $f \in \mathcal{F}_{R}(X)$ with the matrix $\sum_{i=1}^{n} f\left(x_{i}\right) E_{1 i} \in \mathcal{T}_{n}(R)$, which has $\left[f\left(x_{1}\right), \ldots, f\left(x_{n}\right)\right]$ as its first row and zero entries otherwise. Similarly, the embedding $\mathcal{F}_{R}^{\bullet}(X) \subseteq \mathcal{A}_{R}(X) \subseteq \mathcal{T}_{n}(R)$ is given by associating $f \in \mathcal{F}_{R}(X)$ with the matrix $\sum_{i=1}^{n} f\left(x_{i}\right) E_{i n} \in \mathcal{T}_{n}(R)$, which has $\left[f\left(x_{1}\right), \ldots, f\left(x_{n}\right)\right]^{\text {tr }}$ as its last column and zero entries otherwise.

c) Now the Möbius function $\mu=\zeta^{-1} \in \mathcal{A}(X) \subseteq \mathcal{T}_{n}=\mathcal{T}_{n}(\mathbb{Z})$ can be computed in matrix terms, by inverting the matrix associated with $\zeta$. In particular, if $\underline{0} \in X$ is a zero element, then the values $\mu(\underline{0}, ?)$ are given in the first row of the matrix of $\mu$, and if $\underline{1} \in X$ is a one element, then the values $\mu(?, \underline{1})$ are given in the last column of the matrix of $\mu$.

For example, the partially ordered set $X:=[\emptyset,\{1\},\{2\},\{2,3\},\{1,2,3\}]$, see (5.2), yields

$$
\mathcal{A}_{R}(X) \cong\left[\begin{array}{ccccc}
* & * & * & * & * \\
& * & \cdot & \cdot & * \\
& * & * & * \\
& & * & * \\
& & & *
\end{array}\right] \subseteq \mathcal{T}_{5}(R)
$$

The matrices of $\zeta$ and $\mu$ are given as

$$
\zeta \mapsto\left[\begin{array}{ccccc}
1 & 1 & 1 & 1 & 1 \\
& 1 & . & . & 1 \\
& & 1 & 1 & 1 \\
& & & 1 & 1 \\
& & & & 1
\end{array}\right], \quad \mu \mapsto\left[\begin{array}{ccccc}
1 & -1 & -1 & . & 1 \\
& 1 & . & . & -1 \\
& & 1 & -1 & \cdot \\
& & & 1 & -1 \\
& & & & 1
\end{array}\right] .
$$

(8.6) The Möbius algebra. To compute Möbius functions of lattices, the conventional recursive procedure can be adjusted to involve much fewer terms than for partially ordered sets in general. To this end, it suffices to assume only that $X$ is a locally finite partially ordered set having meets and a one element. If $X$ additionally has a zero element, then by local finiteness $X$ is finite, and conversely if $X$ is finite then it has a zero element, thus in this case by the existence of meets $X$ has joins as well, hence is a lattice; see Exercise (20.3).

a) Let $R \neq\{0\}$ be a commutative ring. Then we define Möbius multiplication on $\mathcal{F}_{R}(X)$ as follows: For $f, g \in \mathcal{F}_{R}(X)$ let $f \wedge g: X \rightarrow R: y \mapsto$ $\sum_{x, x^{\prime} \in X, x \wedge x^{\prime}=y} f(x) g\left(x^{\prime}\right)$. From local finiteness and the existence of a one element we infer that this is indeed well-defined. Moreover, we have $f \wedge g=g \wedge f$, and $(f \wedge g) \wedge h=f \wedge(g \wedge h): y \mapsto \sum_{x, x^{\prime}, x^{\prime \prime} \in X, x \wedge x^{\prime} \wedge x^{\prime \prime}=y} f(x) g\left(x^{\prime}\right) h\left(x^{\prime \prime}\right)$, 
for all $f, g, h \in \mathcal{F}_{R}(X)$, that is commutativity and associativity hold. For the map $\delta_{1}: X \rightarrow R: x \mapsto \delta(x, \underline{1})$, where $\delta \in \mathcal{A}_{R}(X)$ is as above, we have $\left(f \wedge \delta_{1}\right)(y)=\sum_{x, x^{\prime} \in X, x \wedge x^{\prime}=y} f(x) \delta\left(x^{\prime}, \underline{1}\right)=f(y)$, for all $y \in X$, hence $\delta_{1} \in \mathcal{A}(X)$ is a neutral element with respect to Möbius multiplication. Since we have distributivity, and $c(f \wedge g)=(c f) \wedge g \in \mathcal{F}_{R}(X)$, for all $c \in R$, we conclude that $\mathcal{F}_{R}(X)$ is a commutative $R$-algebra, called the associated Möbius algebra.

More generally, for $y \in X$ let $\delta_{y}: X \rightarrow R: x \mapsto \delta(x, y)$ and $\mu_{y}: X \rightarrow R: x \mapsto$ $\mu(x, y)$, where $\mu \in \mathcal{A}_{R}(X)$ is the Möbius function of $X$ as above; recall that $\mu(x, y) \in \mathbb{Z}$ for all $x, y \in X$, hence we may consider $\mu$ as a function with values in $R$. Then for any fixed $z \in X$ we have $\sum_{y \leq z} \mu_{y}(x)=\sum_{y \leq z} \mu(x, y)=$ $\sum_{x \leq y \leq z} \mu(x, y)=\delta(x, z)=\delta_{z}(x)$, for all $x \in X$, saying that $\sum_{y \leq z} \mu_{y}=\delta_{z}$; in particular we have $\sum_{y \in X} \mu_{y}=\delta_{\underline{1}}$.

We have $\left(\mu_{y} \wedge \mu_{y^{\prime}}\right)(w)=\sum_{x \leq y, x^{\prime} \leq y^{\prime}, x \wedge x^{\prime}=w} \mu(x, y) \mu\left(x^{\prime}, y^{\prime}\right)$, for $w, y, y^{\prime} \in X$, hence $\left(\mu_{y} \wedge \mu_{y^{\prime}}\right)(w) \neq 0$ only if $w \leq y \wedge y^{\prime}$. In this case, for all $v \leq y \wedge y^{\prime}$ we have $\sum_{v \leq w \leq y \wedge y^{\prime}}\left(\mu_{y} \wedge \mu_{y^{\prime}}\right)(w)=\sum_{v \leq w \leq y \wedge y^{\prime}} \sum_{x \leq y, x^{\prime} \leq y^{\prime}, x \wedge x^{\prime}=w} \mu(x, y) \mu\left(x^{\prime}, y^{\prime}\right)$, hence changing the order of summation the right hand side can be written as $\sum_{v \leq x \leq y, v \leq x^{\prime} \leq y^{\prime}} \mu(x, y) \mu\left(x^{\prime}, y^{\prime}\right)=\sum_{v \leq x \leq y} \mu(x, y) \cdot \sum_{v \leq x^{\prime} \leq y^{\prime}} \mu\left(x^{\prime}, y^{\prime}\right)=\delta_{v, y}$. $\delta_{v, y^{\prime}}$. Thus, if $y \neq y^{\prime}$ then by induction on $l\left(v, y \wedge y^{\prime}\right) \in \mathbb{N}_{0}$ we infer $\left(\mu_{y} \wedge \mu_{y^{\prime}}\right)(v)=$ 0 , and hence $\mu_{y} \wedge \mu_{y^{\prime}}=0 \in \mathcal{F}_{R}(X)$.

Similarly, for $y=y^{\prime}$ we get $\sum_{v \leq w \leq y}\left(\mu_{y} \wedge \mu_{y}\right)(w)=\delta_{v, y}=\sum_{v \leq w \leq y} \mu(w, y)=$ $\sum_{v \leq w \leq y} \mu_{y}(w)$, which by induction on $l(v, y) \in \mathbb{N}_{0}$ entails $\left(\mu_{y} \wedge \mu_{y}\right)(v)=\mu_{y}(v)$, hence $\mu_{y} \wedge \mu_{y}=\mu_{y} \in \mathcal{F}_{R}(X)$. Thus, in conclusion we have $\mu_{y} \wedge \mu_{y^{\prime}}=\delta_{y, y^{\prime}} \mu_{y} \in$ $\mathcal{F}_{R}(X)$, saying that the $\left\{\mu_{y} \in \mathcal{F}_{R}(X) ; y \in X\right\}$ form a decomposition of $\delta_{1}$ into pairwise orthogonal idempotents.

In particular, if $K$ is a field and $X$ is finite, then from $\operatorname{dim}_{K}\left(\mathcal{F}_{K}(X)\right)=|X|$ we infer that $\left\{\mu_{y} \in \mathcal{F}_{K}(X) ; y \in X\right\}$ are the (centrally) primitive idempotents, thus $\mathcal{F}_{K}(X)$ is a split semisimple $K$-algebra of shape $\mathcal{F}_{K}(X) \cong \bigoplus_{x \in X} K$.

b) For $\underline{1} \neq z \in X$ we have $\delta_{z} \wedge \mu_{\underline{1}}=\sum_{y \leq z} \mu_{y} \wedge \mu_{\underline{1}}=0 \in \mathcal{F}_{R}(X)$; note that for $z=\underline{1}$ we just get the trivial formula $\delta_{\underline{1}} \wedge \mu_{\underline{1}}=\sum_{y \in X} \mu_{y} \wedge \mu_{\underline{1}}=\mu_{\underline{1}}$. Thus, we have Weisner's Theorem [1935], saying that for $\underline{1} \neq z \in X$ and all $y \in X$ we have $0=\left(\delta_{z} \wedge \mu_{\underline{1}}\right)(y)=\sum_{w, x \in X, w \wedge x=y} \delta(w, z) \mu(x, \underline{1})=\sum_{x \in X, x \wedge z=y} \mu(x, \underline{1})$.

In particular, if $X$ is finite such that $\underline{0} \neq \underline{1}$, letting $y=\underline{0}$, for $z=\underline{0}$ we recover $\sum_{x \in X} \mu(x, \underline{1})=0$, and whenever $\underline{0} \kappa \underline{1}$ for $\underline{0} \lessdot z$ we get $\sum_{z \not x x} \mu(x, \underline{1})=0$.

Note that, similarly, if $X$ has joins and a zero element, for $\underline{0} \neq z \in X$ and all $y \in X$ we have $\sum_{x \in X, x \vee z=y} \mu(\underline{0}, x)=0$. In particular, again, if $X$ is finite such that $\underline{0} \neq \underline{1}$, letting $y=\underline{1}$, for $z=\underline{0}$ we recover $\sum_{x \in X} \mu(\underline{0}, x)=0$, and whenever $\underline{0} \kappa \underline{1}$ for $z \lessdot \underline{1}$ we get $\sum_{x \not z} \mu(\underline{0}, x)=0$.

(8.7) Möbius functions of lattices. We now proceed to the promised simplification to compute Möbius functions of lattices, where it still suffices to assume that $X$ is a locally finite partially ordered set having meets and a one element.

Let $M \subseteq X \backslash\{\underline{1}\}$ such that for all $\underline{1} \neq x \in X$ there is $y \in M$ such that $x \leq y$; 
in particular $M$ contains all $x \in X$ such that $x \lessdot \underline{1}$. Then for $y \in X$ we have $\delta_{1}-\delta_{y}=\sum_{x \in X} \mu_{x}-\sum_{x \leq y} \mu_{x}=\sum_{x \notin y} \mu_{x}$. Hence, using the fact that the $\mu_{x}$ are pairwise orthogonal idempotents and the special choice of $M$, this yields $\bigwedge_{y \in M}\left(\delta_{\underline{1}}-\delta_{y}\right)=\bigwedge_{y \in M}\left(\sum_{x \not y} \mu_{x}\right)=\sum_{x \not y \text { for all } y \in M} \mu_{x}=\mu_{\underline{1}} \in \mathcal{F}_{R}(X)$. Thus from $\bigwedge_{y \in M}\left(\delta_{\underline{1}}-\delta_{y}\right)=\sum_{L \subseteq M}(-1)^{|L|} \cdot \bigwedge_{y \in L} \delta_{y} \in \mathcal{F}_{R}(X)$, where as usual we define the empty product to be the identity $\delta_{\underline{1}}$, by evaluating at $x \in X$, we get $\mu(x, \underline{1})=\sum_{L \subseteq M}(-1)^{|L|} \cdot\left(\bigwedge_{y \in L} \delta_{y}\right)(x)=\sum_{L \subseteq M, \wedge L=x}(-1)^{|L|}$. In particular, for any $x \in X$ such that $\bigwedge\{y \in X ; y \lessdot \underline{1}\} \not \leq x$ we have $\mu(x, \underline{1})=0$.

If $X$ is finite, then for the zero element we get the cross-cut theorem $\mu(\underline{0}, \underline{1})=$ $\sum_{L \subseteq M, \wedge L=\underline{0}}(-1)^{|L|}$; if $\bigwedge\{x \in X ; x \lessdot \underline{1}\} \neq \underline{0}$ then we have $\mu(\underline{0}, \underline{1})=0$.

Note that, similarly, if $X$ has joins and a zero element, and if $M \subseteq X \backslash\{\underline{0}\}$ is such that for all $\underline{0} \neq x \in X$ there is $y \in M$ such that $y \leq x$, then for all $x \in X$ we have $\mu(\underline{0}, x)=\sum_{L \subseteq M, \bigvee L=x}(-1)^{|L|}$. In particular, for any $x \in X$ such that $x \not \mathbb{\bigvee}\{y \in X ; \underline{0} \lessdot y\}$ we have $\mu(\underline{0}, x)=0$; if $X$ is finite, then $\mu(\underline{0}, \underline{1})=\sum_{L \subseteq M, \bigvee L=\underline{1}}(-1)^{|\bar{L}|}$, and if $\bigvee\{x \in X ; \underline{0} \lessdot x\} \neq \underline{1}$ then $\mu(\underline{0}, \underline{1})=0$.

\section{Möbius functions}

(9.1) Möbius functions of products. a) Let $X^{\prime}$ and $X^{\prime \prime}$ be partially ordered sets. Then the Cartesian product $X:=X^{\prime} \times X^{\prime \prime}$ becomes a partially ordered set, called the direct product of $X^{\prime}$ and $X^{\prime \prime}$, with respect to componentwise comparison, that is we let $x:=\left[x^{\prime}, x^{\prime \prime}\right] \leq\left[y^{\prime}, y^{\prime \prime}\right]=: y \in X$ if and only if $x^{\prime} \leq y^{\prime} \in X^{\prime}$ and $x^{\prime \prime} \leq y^{\prime \prime} \in X^{\prime \prime}$.

Then intervals in $X$ are direct products again, namely $[x, y]=\left[x^{\prime}, y^{\prime}\right] \times\left[x^{\prime \prime}, y^{\prime \prime}\right]$ whenever $x \leq y \in X$. Moreover, $X$ is a lattice if $X^{\prime}$ and $X^{\prime \prime}$ are; $X$ is graded if $X^{\prime}$ and $X^{\prime \prime}$ are; $X$ is locally finite if $X^{\prime}$ and $X^{\prime \prime}$ are; and if $X^{\prime}$ and $X^{\prime \prime}$ are finite then we have $l(X)=l\left(X^{\prime}\right)+l\left(X^{\prime \prime}\right)$.

Let $X^{\prime}$ and $X^{\prime \prime}$ be locally finite. We aim at finding the Möbius function of $X=X^{\prime} \times X^{\prime \prime}$ : Given the Möbius functions $\mu^{\prime}$ and $\mu^{\prime \prime}$ of $X^{\prime}$ and $X^{\prime \prime}$, respectively, we get $\sum_{x \leq z \leq y} \mu^{\prime}\left(x^{\prime}, z^{\prime}\right) \cdot \mu^{\prime \prime}\left(x^{\prime \prime}, z^{\prime \prime}\right)=\sum_{x^{\prime} \leq z^{\prime} \leq y^{\prime}} \sum_{x^{\prime \prime} \leq z^{\prime \prime} \leq y^{\prime \prime}} \mu^{\prime}\left(x^{\prime}, z^{\prime}\right)$. $\mu^{\prime \prime}\left(x^{\prime \prime}, z^{\prime \prime}\right)=\sum_{x^{\prime} \leq z^{\prime} \leq y^{\prime}} \mu^{\prime}\left(x^{\prime}, z^{\prime}\right) \cdot \sum_{x^{\prime \prime} \leq z^{\prime \prime} \leq y^{\prime \prime}} \mu^{\prime \prime}\left(x^{\prime \prime}, z^{\prime \prime}\right)=\delta_{x^{\prime}, y^{\prime}} \cdot \delta_{x^{\prime \prime}, y^{\prime \prime}}=\delta_{x, y}$, for $x, y \in X$, hence the Möbius function of $X$ is given as the product $\mu(x, y)=$ $\mu^{\prime}\left(x^{\prime}, y^{\prime}\right) \cdot \mu^{\prime \prime}\left(x^{\prime \prime}, y^{\prime \prime}\right)$ of the component Möbius functions, for all $x, y \in X$.

b) We consider the direct product $X:=\prod_{i=1}^{k}\left[0, n_{i}\right]$ of the totally ordered intervals $\left[0, n_{i}\right]$, where $n_{1}, \ldots, n_{k} \in \mathbb{N}$ and $k \in \mathbb{N}$. We provide to ways to determine its Möbius function $\mu$, in particular showing that the cross-cut theorem indeed helps to avoid too many explicit computations:

i) Firstly, since $\mu$ is determined locally, it suffices to find $\mu\left(\underline{0},\left[n_{1}, \ldots, n_{k}\right]\right)=$ $\prod_{i=1}^{k} \mu\left(0, n_{k}\right)$. We show that $\mu\left(\underline{0},\left[n_{1}, \ldots, n_{k}\right]\right) \neq 0$ if and only if $n_{i} \leq 1$ for all $i \in\{1, \ldots, k\}$, in which case $\mu\left(\underline{0},\left[n_{1}, \ldots, n_{k}\right]\right)=(-1)^{\epsilon}$, where $\epsilon:=\sum_{i=1}^{k} n_{i}$ : 
For the totally ordered set $[0, n]$, where $n \in \mathbb{N}_{0}$, the matrices of $\zeta$ and $\mu$ are

$$
\zeta \mapsto\left[\begin{array}{ccccc}
1 & 1 & 1 & \ldots & 1 \\
& 1 & 1 & \ldots & 1 \\
& & \ddots & \ddots & \vdots \\
& & & 1 & 1 \\
& & & & 1
\end{array}\right], \quad \mu \mapsto\left[\begin{array}{ccccc}
1 & -1 & & & \\
& 1 & -1 & & \\
& & \ddots & \ddots & \\
& & & 1 & -1 \\
& & & & 1
\end{array}\right]
$$

Hence we have $\mu(0,0)=1$ and $\mu(0,1)=-1$, as well as $\mu(0, n)=0$ for $n \geq 2$. $\sharp$ Note that this also describes the Möbius function of the totally ordered set $\mathbb{N}_{0}$. ii) Secondly, we apply the cross-cut theorem: We have $M:=\left\{\left[m_{1}, \ldots, m_{k}\right] \in\right.$ $\left.X ; \underline{0} \lessdot\left[m_{1}, \ldots, m_{k}\right]\right\}=\left\{e_{1}, \ldots, e_{k}\right\}$, where $e_{i} \in \mathbb{Z}^{k}$ denotes the $i$-th unit vector, and hence $\bigvee M=[1, \ldots, 1] \in X$. Thus we have $\mu\left(\underline{0},\left[m_{1}, \ldots, m_{k}\right]\right)=0$ whenever $\left\{m_{1}, \ldots, m_{k}\right\} \nsubseteq\{0,1\}$. while if $m_{i} \leq 1$ for all $i \in\{1, \ldots, k\}$ then we recover $\mu\left(\underline{0},\left[m_{1}, \ldots, m_{k}\right]\right)=\sum_{L \subseteq M, \bigvee L=\left[m_{1}, \ldots, m_{k}\right]}(-1)^{|L|}=(-1)^{\epsilon}$, where $\epsilon=\left|\left\{e_{i} ; m_{i}=1\right\}\right|=\left|\sum_{i=1}^{k} m_{i}\right|$.

(9.2) Example: Divisibility lattices. a) To determine the Möbius function of $\mathbb{N}$, partially ordered by divisibility |, using the fact that Möbius functions are determined locally, and since for $c \mid d \in \mathbb{N}$ we have $[c, d] \cong\left[\frac{c}{c}, \frac{d}{c}\right] \cong\left[1, \frac{d}{c}\right]$ as partially ordered sets, hence $\mu(c, d)=\mu\left(1, \frac{d}{c}\right)$, it suffices to compute the number-theoretic Möbius function $\mu(n):=\mu(1, n)$ for $n \in \mathbb{N}$ :

If $n=\prod_{i=1}^{k} p_{i}^{n_{i}}$ is the prime factorization of $n$, where $p_{1}, \ldots, p_{k} \in \mathbb{N}$ are pairwise distinct primes and $n_{1}, \ldots, n_{k} \in \mathbb{N}$, for some $k \in \mathbb{N}_{0}$, then we have $c=\prod_{i=1}^{k} p_{i}^{c_{i}}$ $d=\prod_{i=1}^{k} p_{i}^{d_{i}} \mid n$ if and only if $0 \leq c_{i} \leq d_{i} \leq n_{i}$ for all $i \in\{1, \ldots, k\}$, showing that, by going over to the multiplicities of the various primes in the prime factorization of $n$, the interval between 1 and $n$ is isomorphic to the direct product $\prod_{i=1}^{k}\left[0, n_{i}\right]$ of the totally ordered intervals $\left[0, n_{i}\right]$ as partially ordered sets. Hence we have $\mu(n)=0$ if $n_{i} \geq 2$ for some $i \in\{1, \ldots, k\}$, that is if $n$ is not squarefree; and if $n=\prod_{i=1}^{k} p_{i}$ is squarefree then we have $\mu(n)=(-1)^{k}$, where $k$ is the number of distinct prime divisors of $n$. Another related lattice is discussed in Exercise (19.36).

b) We present a few applications of Möbius inversion in number theory: Let $f \in \mathcal{F}(\mathbb{N})$ be a number-theoretic function. Since $\mathbb{N}$ has a zero element, $\mathcal{F}(\mathbb{N})$ becomes a right $\mathcal{A}(\mathbb{N})$-module, hence we get $f^{+}:=f * \zeta \in \mathcal{F}(\mathbb{N})$, where $f^{+}(n)=$ $\sum_{d \mid n} f(d)=\sum_{d \mid n} f\left(\frac{n}{d}\right)$, for all $n \in \mathbb{N}$, and thus $f=f^{+} * \mu \in \mathcal{F}(\mathbb{N})$, where $\left(f^{+} * \mu\right)(n)=\sum_{d \mid n} f^{+}(d) \mu(d, n)=\sum_{d \mid n} f^{+}(d) \mu\left(\frac{n}{d}\right)=\sum_{d \mid n} \mu(d) f^{+}\left(\frac{n}{d}\right)$.

For the constant map $\epsilon \in \mathcal{F}(\mathbb{N})$, given by $\epsilon(n)=1$ for all $n \in \mathbb{N}$, we get $\tau:=\epsilon^{+} \in \mathcal{F}(\mathbb{N})$, where $\tau(n)=\sum_{d \mid n} 1$ is the number of divisors of $n$; this yields $\tau * \mu=\epsilon \in \mathcal{F}(\mathbb{N})$, that is $\sum_{d \mid n} \tau(d) \mu\left(\frac{n}{d}\right)=\sum_{d \mid n} \mu(d) \tau\left(\frac{n}{d}\right)=1$. For

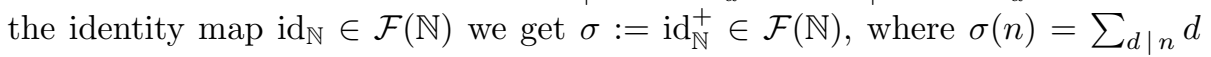


is the sum of the divisors of $n$; this yields $\sigma * \mu=\operatorname{id}_{\mathbb{N}} \in \mathcal{F}(\mathbb{N})$, that is $\sum_{d \mid n} \sigma(d) \mu\left(\frac{n}{d}\right)=\sum_{d \mid n} \mu(d) \sigma\left(\frac{n}{d}\right)=n$.

The other way around, for $n \in \mathbb{N}$ we get $(\epsilon * \mu)(n)=\sum_{d \mid n} \mu\left(\frac{n}{d}\right)=\sum_{d \mid n} \mu(d)=$ $\delta(1, n)=\delta_{1}(n)$, that is $\epsilon * \mu=\delta_{1} \in \mathcal{F}(\mathbb{N})$; this yields $\left(\delta_{1}\right)^{+}=\epsilon \in \mathcal{F}(\mathbb{N})$, that is $\sum_{d \mid n} \delta_{1}(d)=\sum_{d \mid n} \delta_{1}\left(\frac{n}{d}\right)=1$. More interestingly, let $\varphi:=\operatorname{id}_{\mathbb{N}} * \mu \in \mathcal{F}(\mathbb{N})$ be the Euler totient function, where $\varphi(n)=\sum_{d \mid{ }_{n}} d \cdot \mu\left(\frac{n}{d}\right)=\sum_{d \mid n} \mu(d) \cdot \frac{n}{d}$; this yields $\varphi^{+}=\operatorname{id}_{\mathbb{N}} \in \mathcal{F}(\mathbb{N})$, that is $\sum_{d \mid n} \varphi(d)=\sum_{d \mid n} \varphi\left(\frac{n}{d}\right)=n$.

We show that $\varphi(n)$ can be interpreted as a cardinality, and in particular has positive values: Let $\widetilde{\varphi} \in \mathcal{F}(\mathbb{N})$ be given as $\widetilde{\varphi}(n):=\mid\{k \in\{1, \ldots, n\} ; \operatorname{gcd}(k, n)=$ $1\} \mid$. Then we have $\sum_{d \mid n} \widetilde{\varphi}\left(\frac{n}{d}\right)=\sum_{d \mid n}\left|\left\{k \in\left\{1, \ldots, \frac{n}{d}\right\} ; \operatorname{gcd}\left(k, \frac{n}{d}\right)=1\right\}\right|=$ $\sum_{d \mid n}|\{k \in\{1, \ldots, n\} ; \operatorname{gcd}(k, n)=d\}|=\mid \coprod_{d \mid n}\{k \in\{1, \ldots, n\} ; \operatorname{gcd}(k, n)=$ $d\} \mid=n$, implying that $\widetilde{\varphi} * \zeta=\mathrm{id}_{\mathbb{N}}$, and thus $\widetilde{\varphi}=\mathrm{id}_{\mathbb{N}} * \mu=\varphi \in \mathcal{F}(\mathbb{N})$.

We determine $\varphi$ explicitly, using prime factorizations: For $n=\prod_{i=1}^{k} p_{i}^{n_{i}} \in$ $\mathbb{N}$, where $p_{1}, \ldots, p_{k} \in \mathbb{N}$ are pairwise distinct primes, $n_{1}, \ldots, n_{k} \in \mathbb{N}$ and $k \in \mathbb{N}_{0}$, we get $\varphi(n)=\sum_{\left[a_{1}, \ldots, a_{k}\right] \in \prod_{i=1}^{k}\left[0, \ldots, n_{i}\right]}\left(\mu\left(\prod_{i=1}^{k} p_{i}^{a_{i}}\right) \cdot \prod_{i=1}^{k} p_{i}^{n_{i}-a_{i}}\right)=$ $\sum_{\left[\epsilon_{1}, \ldots, \epsilon_{k}\right] \in\{0,1\}^{k}}\left((-1)^{\sum_{i=1}^{k} \epsilon_{i}} \cdot \prod_{i=1}^{k} p_{i}^{n_{i}-\epsilon_{i}}\right)$, which thus implies $\varphi(n)=n$. $\sum_{\left[\epsilon_{1}, \ldots, \epsilon_{k}\right] \in\{0,1\}^{k}} \prod_{i=1}^{k}\left(\frac{-1}{p_{i}}\right)^{\epsilon_{i}}=n \cdot \prod_{i=1}^{k}\left(1-\frac{1}{p_{i}}\right)=\prod_{i=1}^{k} p_{i}^{n_{i}-1}\left(p_{i}-1\right)$.

c) We present a $q$-analogue of the formula $\left(\delta_{1}\right)^{+}=\epsilon \in \mathcal{F}(\mathbb{N})$ : Let $\mathbb{F}_{q}$ be the finite field of order $q$, and let $I_{n}(q) \in \mathbb{N}_{0}$ be the number of monic irreducible polynomials over $\mathbb{F}_{q}$ of degree $n \in \mathbb{N}$. Then since $\mathbb{F}_{q^{d}}$ is the separable splitting field of any irreducible polynomial over $\mathbb{F}_{q}$ of degree $d \in \mathbb{N}$, for $d \in\{1, \ldots, n\}$, and $\mathbb{F}_{q^{d}} \subseteq \mathbb{F}_{q^{n}}$ if and only if $d \mid n$, we get $q^{n}=\sum_{d \mid n} d \cdot I_{d}(q)$

Thus letting $f_{q} \in \mathcal{F}(\mathbb{N})$ be defined by $f_{q}(n):=n \cdot I_{n}(q)$, for $n \in \mathbb{N}$, for $f_{q}^{+} \in \mathcal{F}(\mathbb{N})$ we have $\left(f_{q}^{+}\right)(n)=\sum_{d \mid n} d \cdot I_{d}(q)=q^{n}$, and thus $n \cdot I_{n}(q)=f_{q}(n)=\left(f_{q}^{+} * \mu\right)(n)=$ $\sum_{d \mid n} q^{d} \cdot \mu\left(\frac{n}{d}\right)$, which is a combinatorial formula to compute $I_{n}(q)$.

Indeed, for all $n \in \mathbb{N}$ we may view $f_{q}(n)$ and $f_{q}^{+}(n)$ as a polynomial in $\mathbb{Z}[q]$, hence specializing at $q \mapsto 1$ yields $\left.f_{q}^{+}(n)\right|_{q \mapsto 1}=\left.q^{d}\right|_{q \mapsto 1}=1$, saying that $\left.f_{q}^{+}\right|_{q \mapsto 1}=\epsilon \epsilon$ $\mathcal{F}(\mathbb{N})$. Moreover, we get $\left.f_{q}(n)\right|_{q \mapsto 1}=\sum_{d \mid n} \mu\left(\frac{n}{d}\right)=\sum_{d \mid n} \mu(d, n)=\delta(1, n)$, saying that $\left.f_{q}\right|_{q \mapsto 1}=\delta_{1} \in \mathcal{F}(\mathbb{N})$.

(9.3) Example: Subspace lattices. To compute the Möbius function of $\mathbf{P}_{n}(q)$, where $q$ is a prime power and $n \in \mathbb{N}_{0}$, we observe that for $V \leq W \leq \mathbb{F}_{q}^{n}$ we have $[V, W] \cong[V / V, W / V] \cong\left[\{0\}, \mathbb{F}_{q}^{\operatorname{dim}_{\mathbb{F}_{q}}(W)-\operatorname{dim}_{\mathbb{F}_{q}}(V)}\right]$ as partially ordered sets, hence, since $\mu$ is determined locally, it suffices to determine $\mu_{n}(q):=\mu\left(\{0\}, \mathbb{F}_{q}^{n}\right)$. By induction on $n \in \mathbb{N}$ we show $\mu_{n}(q)=-q^{n-1} \mu_{n-1}(q)$ :

Letting $L \leq \mathbb{F}_{q}^{n}$ be a 1-dimensional $\mathbb{F}_{q}$-subspace, by Weisner's Theorem we have $\sum_{U \leq \mathbb{F}_{q}^{n}, U+L=\mathbb{F}_{q}^{n}} \mu(\{0\}, U)=0$. The condition $U+L=\mathbb{F}_{q}^{n}$ is equivalent to either $U=\mathbb{F}_{q}^{n}$, or $U \lessdot \mathbb{F}_{q}^{n}$ such that $L \not \leq U$. Hence we get $\mu_{n}(q)=\mu\left(\{0\}, \mathbb{F}_{q}^{n}\right)=$ $-\sum_{L \not Z U \ll \mathbb{F}_{q}^{n}} \mu(\{0\}, U)=-\mu_{n-1}(q) \cdot\left|\left\{U \lessdot \mathbb{F}_{q}^{n} ; L \not \leq U\right\}\right|$. Since there are $\frac{q^{n}-1}{q-1}$ 
maximal proper subspaces of $\mathbb{F}_{q}^{n}$, of which $\frac{q^{n-1}-1}{q-1}$ contain $L$, we infer $\mu_{n}(q)=$ $-\mu_{n-1}(q) \cdot\left(\frac{q^{n}-1}{q-1}-\frac{q^{n-1}-1}{q-1}\right)=-q^{n-1} \mu_{n-1}(q)$.

This entails $\mu_{n}(q)=(-1)^{n} q\left(\begin{array}{c}n \\ 2\end{array}\right)$ for $n \in \mathbb{N}_{0}$ : We have $\mu_{0}(q)=1$, and by induction on $n \in \mathbb{N}$ we get $\mu_{n}(q)=-q^{n-1} \mu_{n-1}(q)=-(-1)^{n-1} q^{\left(\begin{array}{c}n-1 \\ 1\end{array}\right)} q^{\left(\begin{array}{c}n-1 \\ 2\end{array}\right)}=(-1)^{n} q^{\left(\begin{array}{c}n \\ 2\end{array}\right)}$. For example, we have $\mu_{1}(q)=-1$ and $\mu_{2}(q)=q$ and $\mu_{3}(q)=-q^{3}$.

Moreover, we observe the following: Considering $\mu_{n}(q)$ as a polynomial in $\mathbb{Z}[q]$, we may specialize at $q \mapsto 1$, yielding $\left.\mu_{n}(q)\right|_{q \mapsto 1}=(-1)^{n}$, for all $n \in \mathbb{N}_{0}$. This coincides with the Möbius function $\mu_{n}$ for the partially ordered set $\mathcal{P}(N)$, where $N$ has cardinality $n$, to be discussed below. Hence $\mu_{n}(q)$ is a $q$-analogue of $\mu_{n}$. Indeed, since we have already noted that $\mathbf{P}_{n}(q)$ can be seen as a $q$-analogue of $\mathcal{P}(N)$, this is not too surprising: Both $\mathbf{P}_{n}(q)$ and $\mathcal{P}(N)$ are graded of rank $n$, and for the respective number of elements of rank $k$, for $k \in\{0, \ldots, n\}$, we have $\left.\left(\begin{array}{l}n \\ k\end{array}\right)_{q}\right|_{q \mapsto 1}=\left(\begin{array}{l}n \\ k\end{array}\right)$. Since Möbius functions in general are determined locally by the recursion $\sum_{x \leq z \leq y} \mu(x, y)=0$ for $x<y$, where $\mu(x, x)=1$, in the present cases it follows that $\left.\mu_{n}(q)\right|_{q \mapsto 1}=\left.\mu\left(\{0\}, \mathbb{F}_{q}^{n}\right)\right|_{q \mapsto 1}=\mu(\emptyset, N)=\mu_{n}$.

(9.4) Example: Subset lattices. a) Let $N$ be a set. To compute the Möbius function of the finitary power sets $\mathcal{P}_{\text {fin }}(N)$ and $\mathcal{P}_{\text {co-fin }}(N)$, since Möbius functions are determined locally, we infer that is suffices to consider the case of a finite set $N$, where we have $\mathcal{P}(N)=\mathcal{P}_{\text {fin }}(N)=\mathcal{P}_{\text {co-fin }}(N)$. Hence let now $N:=\{1, \ldots, n\}$ be a finite set of cardinality $n \in \mathbb{N}_{0}$. Since for $L \subseteq M \subseteq N$ we have $[L, M] \cong[L \backslash L, M \backslash L] \cong[\{\},\{1, \ldots,|M|-|L|\}]$ as partially ordered sets, we conclude that it suffices to compute $\mu_{n}:=\mu(\emptyset, N)$ :

We may identify $\mathcal{P}(N)$ with $\{0,1\}^{n}$ by using indicator functions, that is $M \subseteq N$ is associated with $\left[a_{1}, \ldots, a_{n}\right]$, where $a_{i}=1$ if $i \in M$, and $a_{i}=0$ if $i \in$ $N \backslash M$. Moreover, $\{0,1\}^{n}$ becomes a partially ordered set with respect to the $n$-fold direct product of the totally ordered set $\{0,1\}$ with itself. Then the chosen identification actually is an isomorphism $\mathcal{P}(N) \cong\{0,1\}^{n}$ of partially ordered sets; for the example of $n=3$ see Table 9 . Hence we conclude that $\mu_{n}=\mu(\emptyset, N)=\mu([0, \ldots, 0],[1, \ldots, 1])=(-1)^{n}$. Alternatively, we may proceed by induction on $n \in \mathbb{N}_{0}$ : For $n=0$ we have $\mu_{0}=1$; and for $n \geq 1$ the set $N$ has $\left(\begin{array}{l}n \\ k\end{array}\right)$ subsets of cardinality $k$, for all $k \in\{0, \ldots, n\}$, hence we get $\mu_{n}=-\sum_{k=0}^{n-1}(-1)^{k}\left(\begin{array}{l}n \\ k\end{array}\right)=(-1)^{n}$.

b) Let $N$ be an arbitrary set again, and let $R \neq\{0\}$ be a commutative ring. Then Möbius inversion on $\mathcal{P}_{\text {fin }}(N)$ and $\mathcal{P}_{\text {co-fin }}(N)$ reads as follows:

Since $\mathcal{P}_{\text {fin }}(N)$ has the zero element $\emptyset$, for $f \in \mathcal{F}_{R}\left(\mathcal{P}_{\text {fin }}(N)\right)$ we get $f^{+}(M)=$ $(f * \zeta)(M)=\sum_{L \subseteq M} f(L)$, for all finite $M \subseteq N$. Similarly, $(f * \mu)(M)=$ $\sum_{L \subseteq M} f(L) \mu(L, M)=\sum_{L \subseteq M}(-1)^{|M \backslash L|} f(L)$, for all finite $M \subseteq N$. Thus Möbius inversion yields $f^{+} * \mu=f=(f * \mu)^{+}$for all $f \in \mathcal{F}_{R}\left(\mathcal{P}_{\text {fin }}(N)\right)$.

Since, $\mathcal{P}_{\text {co-fin }}(N)$ has the one element $N$, for $f \in \mathcal{F}_{R}\left(\mathcal{P}_{\text {co-fin }}(N)\right)$ we get $f_{+}(M)=$ $(\zeta * f)(M)=\sum_{M \subseteq L \subseteq N} f(L)$, for all co-finite $M \subseteq N$, and $(\mu * f)(M)=$ 
$\sum_{M \subseteq L \subset N} \mu(M, L) f(L)=\sum_{M \subseteq L \subset N}(-1)^{|L \backslash M|} f(L)$, for all co-finite $M \subseteq N$. Thus Möbius inversion yields $\mu * \bar{f}_{+}=f=(\mu * f)_{+}$for all $f \in \mathcal{F}_{R}\left(\mathcal{P}_{\text {co-fin }}(N)\right)$.

\section{Inclusion-exclusion}

(10.1) Binomial inversion. a) Keeping the notation of (9.4), for finite $N$ and functions $f \in \mathcal{F}_{R}(\mathcal{P}(N))$ only depending on the cardinality of the subsets of $N$, but not on the particular subsets considered, we obtain binomial inversion:

Let $a:=\left[a_{0}, \ldots, a_{n}\right] \in R^{n+1}$, where $n:=|N| \in \mathbb{N}_{0}$, and let $f \in \mathcal{F}_{R}(\mathcal{P}(N))$ be defined by $f(M)=a_{m}$, whenever $M \subseteq N$ such that $|M|=m \in\{0, \ldots, n\}$. Then we have $f^{+}(M)=\sum_{L \subseteq M} f(L)=\sum_{i=0}^{m}\left(\begin{array}{c}m \\ i\end{array}\right) a_{i}=: b_{m}$, saying that $f^{+} \in$ $\mathcal{F}_{R}(\mathcal{P}(N))$ only depends on cardinalities, and is described by $b:=\left[b_{0}, \ldots, b_{n}\right] \in$ $R^{n+1}$. This yields $a_{m}=f(M)=\left(f^{+} * \mu\right)(M)=\sum_{L \subseteq M}(-1)^{|M \backslash L|} \cdot\left(f^{+}\right)(L)=$ $\sum_{L \subseteq M}(-1)^{|M \backslash L|} b_{|L|}=\sum_{j=0}^{m}(-1)^{m-j}\left(\begin{array}{c}m \\ j\end{array}\right) b_{j}$.

For example, the $i$-th unit vector $a:=e_{i} \in R^{n+1}$, where $i \in\{0, \ldots, n\}$, is mapped to $b=\left[b_{0}, \ldots, b_{n}\right]$, where $b_{j}=\left(\begin{array}{l}j \\ i\end{array}\right)$, hence binomial inversion yields $\delta_{i, k}=\sum_{j=0}^{k}(-1)^{k-j}\left(\begin{array}{c}k \\ j\end{array}\right)\left(\begin{array}{l}j \\ i\end{array}\right)$, for $k \in\{0, \ldots, n\}$; in particular, $i=0$ yields $\sum_{j=0}^{k}(-1)^{k-j}\left(\begin{array}{c}k \\ j\end{array}\right)=\delta_{0, k}$. Moreover, for $a:=[1, \ldots, 1]$ we get $b_{j}=\sum_{i=0}^{j}\left(\begin{array}{c}j \\ i\end{array}\right)=$ $(1+1)^{j}=2^{j}$, for $j \in\{0, \ldots, n\}$, thus $b=\left[2^{0}, 2^{1}, \ldots, 2^{n}\right]$; hence binomial inversion yields $1=\sum_{j=0}^{k}(-1)^{k-j}\left(\begin{array}{c}k \\ j\end{array}\right) \cdot 2^{j}$, for $k \in\{0, \ldots, n\}$, where the right hand side indeed coincides with the expansion of $(2-1)^{k}$.

b) We present a couple of more interesting examples: Firstly, we reconsider the number $D_{n}$ of derangements in $\mathcal{S}_{n}$, for $n \in \mathbb{N}_{0}$. Letting still $D_{n, k} \in \mathbb{N}_{0}$ be the number of permutations in $\mathcal{S}_{n}$ having precisely $k \in \mathbb{N}_{0}$ fixed points, choosing any $k$-subset of $\{1, \ldots, n\}$ as set of fixed points, we get $n !=\sum_{k=0}^{n}\left(\begin{array}{l}n \\ k\end{array}\right) D_{n-k}=$ $\sum_{k=0}^{n}\left(\begin{array}{l}n \\ k\end{array}\right) D_{k}$. Thus by binomial inversion we recover $D_{n}=\sum_{k=0}^{n}(-1)^{n-k}\left(\begin{array}{l}n \\ k\end{array}\right)$. $k !=n ! \cdot \sum_{k=0}^{n} \frac{(-1)^{n-k}}{(n-k) !}=n ! \cdot \sum_{k=0}^{n} \frac{(-1)^{k}}{k !}$.

Secondly, we reconsider the Stirling numbers of the second kind; recall that we have $n ! \cdot S_{k, n}=|\operatorname{Surj}(K, N)|$, where $K:=\{1, \ldots, k\}$ and $k \in \mathbb{N}_{0}$. From $\operatorname{Maps}(K, N)=\coprod_{I \subseteq N} \operatorname{Surj}(K, I)$ we get $n^{k}=\sum_{i=0}^{n}\left(\begin{array}{c}n \\ i\end{array}\right) \cdot i ! \cdot S_{k, i}$. Thus by binomial inversion we get $|\operatorname{Surj}(K, N)|=n ! \cdot S_{k, n}=\sum_{j=0}^{n}(-1)^{n-j}\left(\begin{array}{c}n \\ j\end{array}\right) \cdot j^{k}$.

(10.2) Inclusion-exclusion. a) As a further application, we obtain the principle of inclusion-exclusion: Let $X$ be a finite set, and let $X_{1}, \ldots, X_{n} \subseteq X$, where $n \in \mathbb{N}_{0}$. Letting $N:=\{1, \ldots, n\}$ we aim at computing the cardinality $\left|X \backslash \bigcup_{i \in N} X_{i}\right|=\left|\bigcap_{i \in N}\left(X \backslash X_{i}\right)\right| \in \mathbb{N}_{0}$; note that intersections over empty index sets are set equal to $X$ throughout.

For $I \subseteq J \subseteq N$ let $X_{I}:=\bigcap_{i \in I} X_{i}$, and $X_{I \subseteq J}:=X_{I} \cap \bigcap_{j \in J \backslash I}\left(X \backslash X_{j}\right)$; in particular we have $X_{\emptyset \subseteq N}=\bigcap_{j \in N}\left(X \backslash X_{j}\right)$. Hence for $x \in X$ we have $x \in X_{I \subseteq N}$ if and only if $I=\left\{i \in N ; x \in X_{i}\right\}$, and thus $X=\coprod_{I \subseteq N} X_{I \subseteq N}$. We have $X_{I}=$ 
$X_{I} \cap X=\left(\bigcap_{i \in I} X_{i}\right) \cap\left(\coprod_{J \subseteq N \backslash I} X_{J \subseteq N \backslash I}\right)=\coprod_{J \subseteq N \backslash I}\left(\left(\bigcap_{i \in I} X_{i}\right) \cap X_{J \subseteq N \backslash I}\right)=$ $\coprod_{J \subseteq N \backslash I}\left(\left(\bigcap_{i \in I \cup J} X_{i}\right) \cap\left(\bigcap_{j \in N \backslash(I \cup J)}\left(X \backslash X_{j}\right)\right)\right)=\coprod_{I \subseteq J \subseteq N} X_{J \subseteq N}$.

Let $f: \mathcal{P}(N) \rightarrow \mathbb{Z}: I \mapsto\left|X_{I \subseteq N}\right|$. Then we get $\left(f_{+}\right)(I)=\sum_{I \subseteq J \subseteq N}\left|X_{J \subseteq N}\right|=$ $\left|\coprod_{I \subseteq J \subseteq N} X_{J \subseteq N}\right|=\left|X_{I}\right|$, implying that $\left|X_{I \subseteq N}\right|=f(I)=\left(\mu * f_{+}\right)(I)=$ $\sum_{I \subseteq J \subseteq N}(-1)^{|J \backslash I|} \cdot f_{+}(J)=\sum_{I \subseteq J \subseteq N}(-1)^{|J \backslash I|} \cdot\left|X_{J}\right|$, for all $I \subseteq N$. In particular, $I=\emptyset$ yields the set-theoretical inclusion-exclusion formula $\left|\bigcap_{i \in N}\left(X \backslash X_{i}\right)\right|=$ $\sum_{J \subseteq N}(-1)^{|J|} \cdot\left|X_{J}\right|=\sum_{J \subseteq N}(-1)^{|J|} \cdot\left|\bigcap_{j \in J} X_{j}\right| \cdot$

b) If the cardinality $\left|X_{I}\right|$ only depends on the cardinality of $I$, for all $I \subseteq N$, or equivalently if this holds for all the $\left|X_{I \subseteq N}\right|$, then the principle of inclusionexclusion boils down to binomial inversion. Hence the cases of interest here are those where $\left|X_{I}\right|$, and hence $X_{I}$, genuinely depend on $I$. Indeed, the couple of examples in (10.1) is not too interesting in the present context:

Firstly, we reconsider the number $D_{n}$ of derangements in $X:=\mathcal{S}_{n}$. Letting $X_{i}:=\left\{\pi \in \mathcal{S}_{n} ; \pi(i)=i\right\}$, for $i \in N$, for any $I \subseteq N$ we have $\left|X_{I}\right|=\mid\{\pi \in$ $\mathcal{S}_{n} ; \pi(i)=i$ for all $\left.i \in I\right\} \mid=(n-|I|)$ !, and $\left|X_{I \subseteq N}\right|=\left|\left\{\pi \in \mathcal{S}_{n} ; \operatorname{Fix}_{N}(\pi)=I\right\}\right|=$ $D_{n-|I|}$. The principle of inclusion-exclusion says $D_{n-|I|}=\sum_{I \subseteq J \subseteq N}(-1)^{|J \backslash I|}$. $(n-|J|)$ !. Although this entails $D_{n, k}=\left(\begin{array}{l}n \\ k\end{array}\right) \cdot D_{n-k}=\left(\begin{array}{l}n \\ k\end{array}\right) \cdot \sum_{j=0}^{n-k}(-1)^{j}\left(\begin{array}{c}n-k \\ j\end{array}\right)$. $(n-k-j) !=\frac{n !}{k !} \cdot \sum_{j=0}^{n-k} \frac{(-1)^{j}}{j !}$, for $k \in\{0, \ldots, n\}$, it is just equivalent to the case $I=\emptyset$, which is the set-theoretic inclusion-exclusion formula, saying $D_{n}=\sum_{J \subseteq N}(-1)^{|J|} \cdot(n-|J|) !=\sum_{j=0}^{n}(-1)^{j}\left(\begin{array}{c}n \\ j\end{array}\right) \cdot(n-j) !=n ! \cdot \sum_{j=0}^{n} \frac{(-1)^{j}}{j !}$.

Secondly, we reconsider the Stirling numbers of the second kind, or equivalently $|\operatorname{Surj}(K, N)|$, where $K:=\{1, \ldots, k\}$ and $k \in \mathbb{N}_{0}$. Let $X:=\operatorname{Maps}(K, N)$ and $X_{i}:=\left\{f \in X ; f^{-1}(i)=\emptyset\right\}$, for $i \in N$. Then for any $I \subseteq N$ we have $\left|X_{I}\right|=\mid\{f \in$ $\left.X ; f^{-1}(I)=\emptyset\right\}|=| \operatorname{Maps}(K, N \backslash I) \mid=(n-|I|)^{k}$ and $\left|X_{I \subset N}\right|=|\operatorname{Surj}(K, N \backslash I)|$. The principle of inclusion-exclusion says $|\operatorname{Surj}(K, N \backslash I)|=\sum_{I \subseteq J \subseteq N}(-1)^{|J \backslash I|}$. $(n-|J|)^{k}$, which again is equivalent to the case $I=\emptyset$, in which the set-theoretic inclusion-exclusion formula says $|\operatorname{Surj}(K, N)|=\sum_{J \subseteq N}(-1)^{|J|} \cdot(n-|J|)^{k}=$ $\sum_{j=0}^{n}(-1)^{j}\left(\begin{array}{l}n \\ j\end{array}\right) \cdot(n-j)^{k}=\sum_{j=0}^{n}(-1)^{n-j}\left(\begin{array}{l}n \\ j\end{array}\right) \cdot j^{k}$.

c) Here is a more interesting example: For $r \geq 2$ let $P_{r^{\prime}}(n) \subseteq P(n)$ be the set of partitions consisting of parts not divisible by $r$, and let $P_{r \text {-reg }}(n) \subseteq P(n)$ be the set of $r$-regular partitions, that is those all of whose parts have multiplicity less than $r$; for $r=2$ we get the sets $O(n)$ and $D(n)$ of partitions consisting of odd parts and of pairwise distinct parts, respectively, see Table 11. Then we have $\left|P_{r^{\prime}}(n)\right|=\left|P_{r \text {-reg }}(n)\right| \in \mathbb{N}_{0}$ :

For $i \in N$ let $X_{i} \subseteq P(n)=: X$ be the set of partitions having $r i$ as a part, and let $Y_{i} \subseteq P(n)$ be the set of partitions containing the part $i$ at least $r$ times. Then for any subset $I \subseteq N$ we have $\left|\bigcap_{i \in I} X_{i}\right|=\left|P\left(n-\sum_{i \in I} r i\right)\right|=\left|P\left(n-r \cdot \sum_{i \in I} i\right)\right|=$ $\left|\bigcap_{i \in I} Y_{i}\right|$. Hence applying the set-theoretic inclusion-exclusion formula to both $P_{r^{\prime}}(n)=P(n) \backslash \bigcup_{i=1}^{n} X_{i}$ and $P_{r \text {-reg }}(n)=P(n) \backslash \bigcup_{i=1}^{n} Y_{i}$ yields the assertion. \# 
Table 11: Odd-part and distinct-part partitions.

\begin{tabular}{|l||l|l|}
\hline$n$ & $O(n)$ & $D(n)$ \\
\hline \hline 0 & {[]} & {[]} \\
1 & {$[1]$} & {$[1]$} \\
2 & {$\left[1^{2}\right]$} & {$[2]$} \\
3 & {$[3],\left[1^{3}\right]$} & {$[3],[2,1]$} \\
4 & {$[3,1],\left[1^{4}\right]$} & {$[4],[3,1]$} \\
5 & {$[5],\left[3,1^{2}\right],\left[1^{5}\right]$} & {$[5],[4,1],[3,2]$} \\
6 & {$[5,1],\left[3^{2}\right],\left[3,1^{3}\right],\left[1^{6}\right]$} & {$[6],[5,1],[4,2],[3,2,1]$} \\
7 & {$[7],\left[5,1^{2}\right],\left[3^{2}, 1\right],\left[3,1^{4}\right],\left[1^{7}\right]$} & {$[7],[6,1],[5,2],[4,3],[4,2,1]$} \\
\hline
\end{tabular}

(10.3) Grouping index sets. a) We keep the notation of (10.2), and let $s_{k}:=\sum_{J \subseteq N,|J|=k}\left|X_{J}\right| \in \mathbb{N}_{0}$, for $k \in\{0, \ldots, n\}$; in particular, we have $s_{0}=|X|$ and $s_{1}=\sum_{i=1}^{n}\left|X_{i}\right|$. Summing over the subsets of cardinality $k$, the principle of inclusion-exclusion translates as $\sum_{I \subseteq N,|I|=k}\left|X_{I \subseteq N}\right|=\sum_{I \subseteq J \subseteq N,|I|=k}(-1)^{|J|-k}$. $\left|X_{J}\right|=\sum_{l=k}^{n}(-1)^{l-k}\left(\begin{array}{l}l \\ k\end{array}\right) \cdot\left(\sum_{J \subseteq N,|J|=l}\left|X_{J}\right|\right)=\sum_{l=k}^{n}(-1)^{\bar{l}-k}\left(\begin{array}{c}l \\ k\end{array}\right) s_{l}$. In particular, the case $k=0$ allows to reformulate the set-theoretical inclusion-exclusion formula as $\left|\bigcap_{i \in N}\left(X \backslash X_{i}\right)\right|=\sum_{l=0}^{n}(-1)^{l} s_{l}$.

Summing over all subsets of cardinality at least $k$ yields $\sum_{I \subseteq N,|I|>k}\left|X_{I \subseteq N}\right|=$ $\sum_{m=k}^{n} \sum_{l=m}^{n}(-1)^{l-m}\left(\begin{array}{c}l \\ m\end{array}\right) s_{l}=\sum_{l=k}^{n} s_{l} \cdot\left(\sum_{m=k}^{l}(-1)^{l-m}\left(\begin{array}{c}l \\ m\end{array}\right)\right)$. For $k=0$ from $\sum_{m=0}^{l}(-1)^{l-m}\left(\begin{array}{l}l \\ m\end{array}\right)=\delta_{0, l}$ we recover $\sum_{I \subseteq N,|I| \geq 0}\left|X_{I \subseteq N}\right|=\sum_{l=0}^{n} \delta_{0, l} s_{l}=s_{0}=$ $|X|$. Moreover, for $k \geq 1$ the partial alternating row sum formula yields $\sum_{m=k}^{l}(-1)^{l-m}\left(\begin{array}{l}l \\ m\end{array}\right)=\sum_{m=0}^{l-k}(-1)^{m}\left(\begin{array}{l}l \\ m\end{array}\right)=(-1)^{l-k}\left(\begin{array}{l}l-1 \\ l-k\end{array}\right)=(-1)^{l-k}\left(\begin{array}{l}l-1 \\ k-1\end{array}\right)$, from which we get $\sum_{I \subseteq N,|I| \geq k}\left|X_{I \subseteq N}\right|=\sum_{l=k}^{n}(-1)^{l-k}\left(\begin{array}{l}l-1 \\ k-1\end{array}\right) s_{l}$.

b) We show Bonferoni's Theorem [1936], for $k \in\{0, \ldots, n\}$ saying that $(-1)^{k} \cdot \sum_{l=k}^{n}(-1)^{l} s_{l} \geq 0$ :

The set-theoretical inclusion-exclusion formula implying the case $k=0$, we may assume $k \geq 1$. Then we have $\sum_{l=k}^{n}(-1)^{k-l} s_{l}=\sum_{J \subseteq N,|J| \geq k}(-1)^{|J|-k} \cdot\left|X_{J}\right|=$ $\sum_{J \subseteq N,|J| \geq k} \sum_{J \subseteq I \subseteq N}(-1)^{|J|-k} \cdot\left|X_{I \subseteq N}\right|$. Next, changing the order of summation yields $\sum_{l=k}^{n}(-1)^{k-l} s_{l}=\sum_{I \subseteq N,|I| \geq k}\left|X_{I \subseteq N}\right| \cdot\left(\sum_{J \subseteq I,|J| \geq k}(-1)^{|J|-k}\right)=$ $\sum_{I \subseteq N,|I| \geq k}\left|X_{I \subseteq N}\right| \cdot\left(\sum_{j=k}^{|I|}(-1)^{j-k}\left(\begin{array}{c}|I| \\ j\end{array}\right)\right)$. The partial alternating row sum formula yields $\sum_{j=k}^{|I|}(-1)^{j-k}\left(\begin{array}{c}|I| \\ j\end{array}\right)=\sum_{j=0}^{|I|-k}(-1)^{|I|-k-j}\left(\begin{array}{c}|I| \\ j\end{array}\right)=\left(\begin{array}{c}|I|-1 \\ |I|-k\end{array}\right)=\left(\begin{array}{c}|I|-1 \\ k-1\end{array}\right)$, from which we finally get $\sum_{l=k}^{n}(-1)^{k-l} s_{l}=\sum_{I \subseteq N,|I| \geq k}\left|X_{I \subseteq N}\right| \cdot\left(\begin{array}{c}|I|-1 \\ k-1\end{array}\right) \geq 0$. \#

Hence, letting $s:=\left|\bigcap_{i \in N}\left(X \backslash X_{i}\right)\right|=\sum_{l=0}^{n}(-1)^{l} s_{l}$, we have $s \leq \sum_{l=0}^{k}(-1)^{l} s_{l}$ if $k$ is even, and $s \geq \sum_{l=0}^{k}(-1)^{l} s_{l}$ if $k$ is odd. Thus, the partial sums $\sum_{l=0}^{k}(-1)^{l} s_{l}$ successively are lower and upper estimates of $s$; note that the inequalities $s \leq$ $s_{0}=|X|$ and $s \geq s_{0}-s_{1}=|X|-\sum_{i=1}^{n}\left|X_{i}\right|$ are obvious anyway. 
(10.4) Improving inclusion-exclusion. We get an improvement of the principle of inclusion-exclusion by taking the intersection configuration into account. To do so, keeping the notation of (10.2), we consider the set $\mathcal{X}:=\left\{X_{I} \in\right.$ $\mathcal{P}(N) ; I \subseteq N\}$, partially ordered by set-theoretic inclusion $\subseteq$, and having zeta and Möbius functions $\zeta \in \mathcal{A}(\mathcal{X})$ and $\mu \in \mathcal{A}(\mathcal{X})$, respectively.

For $M \in \mathcal{X}$ let $\widehat{M}:=M \backslash \bigcup\{L \in \mathcal{X} ; L \subset M\}=\bigcap\{M \backslash L ; L \in \mathcal{X}, L \subset M\} \subseteq X$ be the set of all elements of $M$ which are not contained in a strictly smaller set in $\mathcal{X}$; hence we have $M=\coprod_{L \subseteq M \in \mathcal{X}} \widehat{L}$. In particular, we have $X=X_{\emptyset} \in \mathcal{X}$ and $\widehat{X}=X \backslash \bigcup_{i \in N} X_{i}=\bigcap_{i \in N}\left(X \backslash X_{i}\right)$, where our aim hence is to compute $|\widehat{X}|$.

Let now $f: \mathcal{X} \rightarrow \mathbb{Z}: M \mapsto|\widehat{M}|$. Then for $f^{+}=f * \zeta \in \mathcal{F}(\mathcal{X})$ we have $f^{+}(M)=$ $\sum_{L \subseteq M \in \mathcal{X}} f(L)=\sum_{L \subseteq M \in \mathcal{X}}|\widehat{L}|=\left|\coprod_{L \subseteq M \in \mathcal{X}} \widehat{L}\right|=|M|$, for all $M \in \mathcal{X}$. Thus we indeed get a shorter inclusion-exclusion formula $|\widehat{X}|=f(X)=\left(f^{+} * \mu\right)(X)=$ $\sum_{L \in \mathcal{X}} f^{+}(L) \cdot \mu(L, X)=\sum_{L \in \mathcal{X}} \mu(L, X) \cdot|L|$, of course at the expense of having to compute the Möbius function $\mu$ of $\mathcal{X}$.

For example, for $n=3$, let $A, B, C \subseteq X$ such that $A \cap B=A \cap C=B \cap C$; hence $A \cap B \cap C=A \cap B$ as well. Then the set-theoretic inclusion-exclusion formula yields $|X \backslash(A \cup B \cup C)|=|X|-|A|-|B|-|C|+|A \cap B|+|A \cap C|+$ $+|B \cap C|-|A \cap B \cap C|=|X|-|A|-|B|-|C|+2 \cdot|A \cap B \cap C|$. Now we have $\mathcal{X}=\{A \cap B \cap C, A, B, C, X\} \cong\{\emptyset,\{1\},\{2\},\{3\},\{1,2,3\}\} \cong \mathbf{P}_{2}(2)$ as partially ordered sets; hence the matrices of $\zeta$ and $\mu$ are

$$
\zeta \mapsto\left[\begin{array}{ccccc}
1 & 1 & 1 & 1 & 1 \\
& 1 & . & . & 1 \\
& & 1 & . & 1 \\
& & & 1 & 1 \\
& & & & 1
\end{array}\right], \quad \mu \mapsto\left[\begin{array}{ccccc}
1 & -1 & -1 & -1 & 2 \\
& 1 & . & \cdot & -1 \\
& & 1 & . & -1 \\
& & & 1 & -1 \\
& & & & 1
\end{array}\right]
$$

Reading off the last column of the matrix of $\mu$ yields again $|X \backslash(A \cup B \cup C)|=$ $|X|-|A|-|B|-|C|+2 \cdot|A \cap B \cap C|$.

(10.5) Example: Problème des ménages. How many ways are there to place $n \geq 2$ couples at a circular table, such that men and women are in alternate places, but such that no husband sits at either side of his wife? Allowing for arbitrary renumbering of the couples, and for exchanging the roles of men and women, up to a factor of $2 \cdot n$ ! the number sought is given as follows: We may assume that women 1 to $n$ are seated in clockwise order. Numbering the places for the men in clockwise order as well, where seat 1 is the one left to woman 1 , we have to determine the number $m_{n} \in \mathbb{N}_{0}$ of all discordant permutations $\pi \in \mathcal{S}_{n}$ such that $\pi(i) \not \equiv i, i+1(\bmod n)$ for all $i \in N:=\{1, \ldots, n\}$.

To this end, we apply the principle of inclusion-exclusion to the sets $X_{i}:=\{\pi \in$ $\mathcal{S}_{n} ; \pi(i)=i$ or $\left.\pi(i) \equiv i+1(\bmod n)\right\}$, for $i \in N$. Since in this case $\left|X_{I}\right|$ indeed depends on the particular choice of $I \subseteq N$, not just on $|I|$ alone, we are going to determine $\sum_{I \subseteq N,|I|=k}\left|X_{I}\right|$, for $k \in\{0, \ldots, n\}$, in a single step: 
To do so, we consider the permutation matrices associated with $\mathcal{S}_{n}$, that is the $(n \times n)$-matrices with entries in $\{0,1\}$, such that any row and any column contains precisely one entry 1 . Then, given $\pi \in \mathcal{S}_{n}$, we have $\pi \in X_{I}$, for some $I \subseteq N$, if and only if the entries 1 in the columns indexed by $I$ are amongst the positions marked by ' $*$ ' in the following $(n \times n)$-matrix, while after fixing the entries in the columns indexed by $I$ for the columns indexed by $N \backslash I$ all $(n-|I|)$ ! possible choices left are allowed:

$$
\left[\begin{array}{ccccc}
* & \cdot & \cdot & \ldots & * \\
* & * & \cdot & & \cdot \\
\cdot & * & * & & \cdot \\
\vdots & & \ddots & \ddots & \vdots \\
\cdot & \cdot & \ldots & * & *
\end{array}\right]
$$

Allowing for all subsets $I \subseteq N$ of a fixed cardinality $k$, the fact that any row and column of the above matrix contains at most one entry 1 translates into the task to determine the number of ways to choose $k$ pairwise non-adjacent positions on a circle of length $2 n$.

To do so, we consider a circular array with positions $\{1, \ldots, m\}$, where $m \in \mathbb{N}$, which has to be filled with $k \leq\left\lfloor\frac{m}{2}\right\rfloor$ entries ' $\mid$ ' and $m-k$ entries ' $\bullet$ ', such that the entries '|' are pairwise non-adjacent. Thinking of the $m-k$ entries ' $\bullet$ ' to be arranged already, the valid configurations are described by the $k$-subset of the $m-k$ spaces between the entries ' $\bullet$ ' hosting the entries ' $\mid$ '. In order to count these configurations, we distinguish the cases which entry occupies position 1:

If this is an entry ' $\bullet$ ', we may choose any $k$-subset of the $m-k$ spaces; if $k \geq 1$ and this is an entry ' $\mid$ ', then there are only $m-k-1$ spaces left, from which we may choose any $(k-1)$-subset. Thus we get a total of $\left(\begin{array}{c}m-k \\ k\end{array}\right)+\left(\begin{array}{c}m-k-1 \\ k-1\end{array}\right)=$ $\left(\begin{array}{c}m-k \\ k\end{array}\right)+\frac{k}{m-k} \cdot\left(\begin{array}{c}m-k \\ k\end{array}\right)=\frac{m}{m-k} \cdot\left(\begin{array}{c}m-k \\ k\end{array}\right)$ possibilities; this also holds for $k=0$.

Thus we get $\sum_{I \subseteq N,|I|=k}\left|X_{I}\right|=(n-k) ! \cdot \frac{2 n}{2 n-k} \cdot\left(\begin{array}{c}2 n-k \\ k\end{array}\right)$. Hence the set-theoretic inclusion-exclusion formula says that $m_{n}=\sum_{I \subseteq N}(-1)^{|I|} \cdot\left|X_{I}\right|=\sum_{k=0}^{n}(-1)^{k}$. $\left(\sum_{I \subseteq N,|I|=k}\left|X_{I}\right|\right)=\sum_{k=0}^{n} \frac{(-1)^{k} \cdot(n-k) ! \cdot 2 n}{2 n-k} \cdot\left(\begin{array}{c}2 n-k \\ k\end{array}\right)$ [Touchard, 1934]. Singling out the case $k=n$, index shifting, and simplifying the summands yields $m_{n}=$ $(-1)^{n} \cdot 2+n \cdot \sum_{k=1}^{n}(-1)^{k-1} \cdot(n-k) ! \cdot\left(\begin{array}{c}2 n-k \\ k-1\end{array}\right)$. For example, we have $m_{2}=0$ $m_{3}=1, m_{4}=2, m_{5}=13, m_{6}=80, m_{7}=579, m_{8}=4738, m_{9}=439792$.

\section{Finite incidence algebras}

We consider finite dimensional incidence algebras from the perspective of representation theory; for more on the background needed see [3, Ch.I-III].

(11.1) Finite dimensional incidence algebras. Keeping the notation of (8.5), let $X$ be a finite partially ordered set, and let $\mathcal{A}:=\mathcal{A}_{K}(X)=\left\langle E_{i j} ; x_{i} \leq\right.$ $\left.x_{j}\right\rangle_{K} \subseteq \mathcal{T}_{n}(K)$, where $K$ is a field. 
Then we have $\mathcal{J}:=\mathcal{J}_{K}(X)=\mathcal{U}_{K}(X)=\left\langle E_{i j} ; x_{i}<x_{j}\right\rangle_{K}$. Hence we have $\mathcal{A} / \mathcal{J} \cong$ $\bigoplus_{i=1}^{n} K$, thus $\mathcal{A}$ is split and has precisely $n$ simple modules $\left\{S_{1}, \ldots, S_{n}\right\}$, up to isomorphism. We have $\operatorname{dim}_{K}\left(S_{i}\right)=1$, for $i \in\{1, \ldots, n\}$, hence $\mathcal{A}$ is a basic algebra. Moreover, we get $\mathcal{J}^{2}=\left\langle E_{i k} ; x_{i}<x_{j}<x_{k} \text { for some } j\right\rangle_{K}$, and thus $\mathcal{J} / \mathcal{J}^{2} \cong\left\langle E_{i j} ; x_{i} \lessdot x_{j}\right\rangle_{K}$. The radical length of $\mathcal{A}$ equals $l(X)+1$, that is $\mathcal{J}^{k}=\{0\}$ if and only if $k \geq l(X)+1$; note that $l(X) \leq n-1$.

Next, $E_{n}=\sum_{i=1}^{n} E_{i i} \in \mathcal{A}$ is a decomposition into pairwise orthogonal primitive idempotents. We assume notation chosen such that $E_{i i}$ acts as the identity on $S_{i}$; hence $S_{j} E_{i i}=\{0\}$ whenever $i \neq j \in\{1, \ldots, n\}$. Then the projective cover of $S_{i}$ is given as $P_{i}=E_{i i} \mathcal{A}$, and we have $\mathcal{A} \cong \bigoplus_{i=1}^{n} P_{i}$. Using the notation of (8.3), we have $P_{i}=E_{i i} \mathcal{A}=\mathcal{I}_{K}\left(\left\{x_{i}\right\}, X\right)=\left\langle E_{i j} ; x_{i} \leq x_{j}\right\rangle_{K}$, for $i \in\{1, \ldots, n\}$, thus $\operatorname{dim}_{K}\left(P_{i}\right)=\left|\left\{j \in\{1, \ldots, n\} ; x_{i} \leq x_{j}\right\}\right|=\left|\left\langle x_{i} \leq\right\rangle\right|$, where $\left\langle x_{i} \leq\right\rangle \subseteq X$ denotes the principal coideal generated by $x_{i}$.

Given $j \in\{1, \ldots, n\}$ such that $x_{i} \leq x_{j}$, the $K$-linear map $\alpha_{i j}: P_{j} \rightarrow P_{i}: E_{j k} \rightarrow$ $E_{i k}$, for all $k \in\{1, \ldots, n\}$ such that $x_{j} \leq x_{k}$, is an embedding of $\mathcal{A}$-modules, where $\alpha_{i j}\left(P_{j}\right)=\left\langle E_{i k} ; x_{j} \leq x_{k}\right\rangle_{K}=\mathcal{I}_{K}\left(\left\{x_{i}\right\},\left\langle x_{j} \leq\right\rangle\right)$. Indeed, a consideration of matrices shows that applying $\alpha_{i j}$ amounts to left multiplication with $E_{i j}$, hence $\alpha_{i j}\left(P_{j}\right)=E_{i j} \cdot E_{j j} \mathcal{A}=E_{i j} \mathcal{A}$, and $\alpha_{i j} \circ \alpha_{j k}=\alpha_{i k}$ whenever $x_{i} \leq x_{j} \leq x_{k}$.

Hence $S_{j}$ is a constituent of $P_{i}$ whenever $x_{i} \leq x_{j}$, and a comparison with $\operatorname{dim}_{K}\left(P_{i}\right)$ shows that it occurs with multiplicity $\left[P_{i}: S_{j}\right]=1$ if $x_{i} \leq x_{j}$, and $\left[P_{i}: S_{j}\right]=0$ otherwise; thus $P_{i}$ is multiplicity-free. Thus in turn we have $\operatorname{Hom}_{\mathcal{A}}\left(P_{j}, P_{i}\right)=\left\langle\alpha_{i j}\right\rangle_{K}$ whenever $x_{i} \leq x_{j}$, and $\operatorname{Hom}_{\mathcal{A}}\left(P_{j}, P_{i}\right)=\{0\}$ otherwise; in particular, $P_{i}$ is simple if and only if $x_{i} \in X$ is maximal.

Thus the Cartan matrix $\left[\left[P_{i}: S_{j}\right]\right]_{i j} \in \mathbb{Z}^{n \times n}$ of $\mathcal{A}$ is coincides with the matrix of the zeta function in $\mathcal{A}$. In particular, we conclude that the partially ordered set $X$ can be recovered from its incidence algebra $\mathcal{A}$, in other words two incidence algebras are isomorphic if and only if the underlying partially ordered sets are.

(11.2) Path algebra description. We proceed to describe $\mathcal{A}$ as a path algebra quotient. To do so, we first determine the Ext quiver of $\mathcal{A}$, which has the simple $\mathcal{A}$-modules $\left\{S_{1}, \ldots, S_{n}\right\}$ as its vertices, and $\left[\operatorname{rad}\left(P_{i}\right) / \operatorname{rad}^{2}\left(P_{i}\right): S_{j}\right]$ arrows $S_{j} \leftarrow S_{i}$. Actually, the latter coincides with the Hasse diagram $\widehat{X}$ of $X$; note that $\widehat{X}$ only has simple arrows and is acyclic, that is without oriented loops:

We have $\operatorname{rad}\left(P_{i}\right)=E_{i i} \mathcal{A} \cdot \mathcal{J}=E_{i i} \mathcal{J}=\left\langle E_{i j} ; x_{i}<x_{j}\right\rangle_{K}$ and $\operatorname{rad}^{2}\left(P_{i}\right)=E_{i i} \mathcal{J}^{2}=$ $\left\langle E_{i k} ; x_{i}<x_{j}<x_{k} \text { for some } j\right\rangle_{K}$, thus $\operatorname{rad}\left(P_{i}\right) / \operatorname{rad}^{2}\left(P_{i}\right) \cong\left\langle E_{i j} ; x_{i} \lessdot x_{j}\right\rangle_{K}$. Now we have $\alpha_{i j}\left(P_{j}\right) \leq \operatorname{rad}\left(P_{i}\right)$ whenever $x_{i}<x_{j}$, which entails $\alpha_{i k}\left(P_{k}\right)=$ $\alpha_{i j} \alpha_{j k}\left(P_{k}\right) \leq \operatorname{rad}^{2}\left(P_{i}\right)$ whenever $x_{i}<x_{j}<x_{k}$, saying that in this case $S_{k}$ is not a constituent of $\operatorname{rad}\left(P_{i}\right) / \operatorname{rad}^{2}\left(P_{i}\right)$. Comparing with the $K$-dimension of $\operatorname{rad}\left(P_{i}\right) / \operatorname{rad}^{2}\left(P_{i}\right)$ we thus infer that indeed $\operatorname{rad}\left(P_{i}\right) / \operatorname{rad}^{2}\left(P_{i}\right) \cong \bigoplus_{j ; x_{i} \lessdot x_{j}} S_{j} \quad \sharp$

Let $\widehat{\mathcal{A}}$ be the path algebra associated with $\widehat{X}:$ A $K$-basis of $\widehat{\mathcal{A}}$ is given by the set of all oriented paths $\rho=\left(x_{i_{k}} \leftarrow x_{i_{k-1}} \leftarrow \cdots \leftarrow x_{i_{1}} \leftarrow x_{i_{0}}\right)$ of length $k \in \mathbb{N}_{0}$ in $\widehat{X}$; in particular, this encompasses the empty paths $\rho_{i}$ at each vertex 
$x_{i}$, and the paths $\rho_{i j}:=\left(x_{i} \leftarrow x_{j}\right)$ whenever $x_{i} \lessdot x_{j}$. Here, $s(\rho)=i_{0}$ and $t(\rho)=i_{k}$ are called the source and target vertices of $\rho$, respectively. For paths $\sigma, \rho \in \widehat{\mathcal{A}}$, the product of $\sigma \rho$ is defined as the concatenation (to the left) of $\sigma$ and $\rho$ whenever $s(\sigma)=t(\rho)$, otherwise let $\sigma \cdot \rho:=0$; we have $\rho \rho_{i}=\rho$ if $s(\rho)=i$, and $\rho_{j} \rho=\rho$ if $t(\rho)=j$. Hence $\widehat{\mathcal{A}}$ is generated as a $K$-algebra by $\left\{\rho_{1}, \ldots, \rho_{n}\right\} \dot{\cup}\left\{\rho_{i j} ; x_{i} \lessdot x_{j}\right\}$, and since $\widehat{X}$ is acyclic $\widehat{\mathcal{A}}$ is finite dimensional.

Now, the concatenation rule for paths and the multiplication rule for the matrices $E_{i j}$ entail that $\pi: \widehat{\mathcal{A}} \rightarrow \mathcal{A}: \rho_{i} \mapsto E_{i i}, \rho_{i j} \mapsto E_{i j}$, whenever $x_{i} \lessdot x_{j}$, extends to an epimorphism of $K$-algebras. We determine a finite set of defining relations for $\mathcal{A}$ as a quotient of $\widehat{\mathcal{A}}$, that is an ideal generating set of $\operatorname{ker}(\pi) \unlhd \widehat{\mathcal{A}}$ :

If $\rho=\left(x_{i_{k}} \leftarrow x_{i_{k-1}} \leftarrow \cdots \leftarrow x_{i_{1}} \leftarrow x_{i_{0}}\right) \in \widehat{\mathcal{A}}$ is a path, then we have $\pi(\rho)=$ $E_{i_{k} i_{k-1}} E_{i_{k-1} i_{k-2}} \cdots E_{i_{1} i_{0}}=E_{i_{k} i_{0}}=E_{t(\alpha), s(\alpha)} \in \mathcal{A}$. Hence, if $\sigma, \rho \in \widehat{\mathcal{A}}$ are paths such that $s(\sigma)=s(\rho)$ and $t(\sigma)=t(\rho)$, then we have $\pi(\sigma)=\pi(\rho)$. Thus, letting $\mathcal{K} \unlhd \widehat{\mathcal{A}}$ be the ideal generated by all differences $\sigma-\rho$ of paths such that $s(\sigma)=s(\rho)$ and $t(\sigma)=t(\rho)$, we have $\mathcal{K} \subseteq \operatorname{ker}(\pi)$. Conversely, choosing a path $x_{i} \leftarrow \cdots \leftarrow$ $x_{j}$, for any pair $[i, j]$ such that $x_{i} \leq x_{j}$, yields a $K$-generating set of $\widehat{\mathcal{A}} / \mathcal{K}$ of cardinality $\left|\left\{[i, j] ; x_{i} \leq x_{j}\right\}\right|=\operatorname{dim}_{K}\left(\left\langle E_{i j} ; x_{i} \leq x_{j}\right\rangle_{K}\right)=\operatorname{dim}_{K}(\mathcal{A})$. Hence we conclude that $\operatorname{dim}_{K}(\widehat{\mathcal{A}} / \operatorname{ker}(\pi)) \leq \operatorname{dim}_{K}(\widehat{\mathcal{A}} / \mathcal{K}) \leq \operatorname{dim}_{K}(\mathcal{A})=\operatorname{dim}_{K}(\widehat{\mathcal{A}} / \operatorname{ker}(\pi))$, implying equality throughout, and thus $\operatorname{ker}(\pi)=\mathcal{K}$.

Note that since for any $i$ there is only one empty path at $x_{i}$, namely $\rho_{i}$, and for any $x_{i} \lessdot x_{j}$ there only one path from $x_{j}$ to $x_{i}$, namely $\rho_{i j}$, all paths involved in elements of $\operatorname{ker}(\pi)$ have length at least 2 , saying that $\operatorname{ker}(\pi)$ indeed is an admissible ideal, that is $\operatorname{ker}(\pi) \subseteq \operatorname{rad}^{2}(\widehat{\mathcal{A}})$; note that $\operatorname{rad}^{k}(\widehat{\mathcal{A}})$ has the set of all paths of length at least $k$ as a $K$-basis, for $k \in \mathbb{N}_{0}$.

(11.3) Projective modules. a) We proceed to examine the submodule lattice $\mathcal{M}\left(P_{i}\right)$ of $P_{i}$, using the terminology introduced in (7.8): Since $P_{i}$ is multiplicityfree, its submodule lattice $\mathcal{M}\left(P_{i}\right)$ is distributive, in particular finite, being described by the set of ideals of $\mathcal{L}\left(P_{i}\right)$, where for any $j$ such that $x_{i} \leq x_{j}$ there is a unique $S_{j}$-local submodule. In this case, since $P_{j}$ is $S_{j}$-local and $\alpha_{i j}$ is an embedding, we conclude that $P_{j} \cong \alpha_{i j}\left(P_{j}\right)=E_{i j} \mathcal{A} \leq E_{i i} \mathcal{A}=P_{i}$ is the $S_{j}$-local submodule of $P_{i}$. In particular, any local submodule of $P_{i}$ is projective.

Hence the submodules of $P_{i}=\mathcal{I}_{K}\left(\left\{x_{i}\right\}, X\right)$ are precisely given as $\mathcal{I}_{K}\left(\left\{x_{i}\right\}, Y\right)$, where $Y$ runs through the coideals of $X$ contained in the principal coideal $\left\langle x_{i} \leq\right\rangle$. Thus the submodule lattice of $P_{i}$ is naturally isomorphic to the lattice of coideals mentioned, where the latter is partially ordered by set-theoretic inclusion. In particular, for the socle of $P_{i}$ we have $\operatorname{soc}\left(P_{i}\right) \cong \bigoplus_{j ; x_{i} \leq x_{j} \in X \text { maximal }} S_{j}$.

Moreover, the blocks of $\mathcal{A}$, that is the smallest direct summands of $\mathcal{A}$ as a $K$ algebra, are given by the connected components of $X$, that is the connected components of the unoriented graph underlying the Hasse diagram $\widehat{X}$ of $X$; in particular, $\mathcal{A}$ is a block if and only if $X$ is connected.

b) We show that all submodules of $P_{i}$ are projective, not just the local ones, 
if and only if for all $x_{j} \geq x_{i}$ there is a unique path in the Hasse diagram $\widehat{X}$ between $x_{j}$ and $x_{i}$, that is a unique saturated chain in $X$ between $x_{i}$ and $x_{j}$ :

Assume that there are distinct paths bewtween $x_{j}$ and $x_{i}$. Then there are $x_{k}$ and $x_{l}$ such that $x_{i} \leq x_{k} \leq x_{j}$ and $x_{i} \leq x_{l} \leq x_{j}$, but $x_{k} \not \leq x_{l} \not \leq x_{k}$. Thus we have $\alpha_{i k}\left(P_{k}\right) \leq P_{i}$ and $\alpha_{i l}\left(P_{l}\right) \leq P_{i}$, such that $\alpha_{i k}\left(P_{k}\right) \not \leq \alpha_{i l}\left(P_{l}\right) \not \leq \alpha_{i k}\left(P_{k}\right)$ and $\alpha_{i j}\left(P_{j}\right) \leq \alpha_{i k}\left(P_{k}\right) \cap \alpha_{i l}\left(P_{l}\right)$, thus $U:=\alpha_{i k}\left(P_{k}\right)+\alpha_{i l}\left(P_{l}\right)$ fulfills $U / \operatorname{rad}(U) \cong$ $S_{k} \oplus S_{l}$, but its constituent $S_{j}$ has multiplicity 1 , hence $U$ is not projective.

Conversely, assume that the above uniqueness property holds, let $U \leq P_{i}$ be a submodule, and let $Y=\left\{x_{j} \in X ; \alpha_{i j}\left(P_{j}\right) \leq U\right\} \subseteq\left\langle x_{i} \leq\right\rangle \subseteq X$ be the associated coideal of $X$. Then letting $x_{j_{1}}, \ldots, x_{j_{r}} \in Y$ be the minimal elements of $Y$, we have $U=\sum_{k=1}^{r} \alpha_{i, j_{k}}\left(P_{j_{k}}\right)$, where $r=r(U) \in \mathbb{N}_{0}$ coincides with the rank of $U \in \mathcal{M}\left(P_{i}\right)$. Thus, by the uniqueness property assumed, for any $x_{j} \in Y$ there is a unique $k=k(j) \in\{1, \ldots, r\}$ such that $x_{j_{k}} \leq x_{j}$, implying that for any constituent $S_{j}$ of $U$ there is a unique $k=k(j)$ such that $S_{j}$ is a constituent of $\alpha_{i, j_{k}}\left(P_{j_{k}}\right)$. Hence we infer that $\alpha_{i, j_{k}}\left(P_{j_{k}}\right) \cap \sum_{l \neq k} \alpha_{i, j_{l}}\left(P_{j_{l}}\right)=\{0\}$ for all $k \in\{1, \ldots, r\}$, that is $U=\bigoplus_{k=1}^{r} \alpha_{i, j_{k}}\left(P_{j_{k}}\right) \cong \bigoplus_{k=1}^{r} P_{j_{k}}$ is projective. $\quad \sharp$ In particular, in this case $\operatorname{rad}\left(P_{i}\right)=\sum_{x_{i} \lessdot x_{j}} \alpha_{i, j}\left(P_{j}\right)=\bigoplus_{x_{i} \lessdot x_{j}} \alpha_{i, j}\left(P_{j}\right) \cong$ $\bigoplus_{x_{i} \ll x_{j}} P_{j}$ is projective. Moreover, running over all $i \in\{1, \ldots, n\}$, we conclude that any submodule of any projective-indecomposable module is projective again, if and only if all connected components of $X$ are trees; in particular, in this case $\mathcal{J}=\bigoplus_{i=1}^{n} \operatorname{rad}\left(P_{i}\right) \cong \bigoplus_{i=1}^{n}\left(\bigoplus_{x_{i} \lessdot x_{j}} P_{j}\right)$ is projective.

For example, let $X:=[\emptyset,\{1\},\{2\},\{2,3\},\{1,2,3\}]$ be partially ordered by settheoretic inclusion $\subseteq$, whose Cartan matrix is given as the matrix of its zeta function in (8.5). Then we have $\operatorname{rad}\left(P_{\emptyset}\right)=\alpha_{\emptyset,\{1\}}\left(P_{\{1\}}\right)+\alpha_{\emptyset,\{2\}}\left(P_{\{2\}}\right)$, where $\alpha_{\emptyset,\{1\}}\left(P_{\{1\}}\right) \cap \alpha_{\emptyset,\{2\}}\left(P_{\{2\}}\right)=\alpha_{\emptyset,\{1,2,3\}}\left(P_{\{1,2,3\}}\right)=\operatorname{soc}\left(P_{\emptyset}\right) \cong S_{\{1,2,3\}}$, hence $\operatorname{rad}\left(P_{\emptyset}\right)$ is not projective.

(11.4) Injective modules. a) Using the projective cover $P_{i}=E_{i i} \mathcal{A}$ of $S_{i}$, its $\mathcal{A}$-dual $P_{i}^{\vee}:=\operatorname{Hom}_{\mathcal{A}}\left(P_{i}, \mathcal{A}\right) \cong \operatorname{Hom}_{\mathcal{A}}\left(E_{i i} \mathcal{A}, \mathcal{A}\right) \cong \mathcal{A} E_{i i}=\left\langle E_{j i} ; x_{j} \leq x_{i}\right\rangle_{K}=$ $\mathcal{I}_{K}\left(X,\left\{x_{i}\right\}\right)$ is a projective left $\mathcal{A}$-module; hence we have $\operatorname{dim}_{K}\left(P_{i}^{\vee}\right)=\mid\{j \in$ $\left.\{1, \ldots, n\} ; x_{j} \leq x_{i}\right\}|=|\left\langle\leq x_{i}\right\rangle \mid$, where $\left\langle\leq x_{i}\right\rangle \subseteq X$ denotes the principal ideal generated by $x_{i}$. Letting $\left\{T_{1}, \ldots, T_{n}\right\}$ be the simple left $\mathcal{A}$-modules, where we assume notation chosen such that $E_{i i}$ acts as the identity on $T_{i}$, we conclude that $P_{i}^{\vee}$ is the projective cover of $T_{i}$. Analogous to the right module case we infer that $\left[P_{i}^{\vee}: T_{j}\right]=1$ if $x_{j} \leq x_{i}$, and $\left[P_{i}^{\vee}: T_{j}\right]=0$ otherwise.

The submodules of $P_{i}^{\vee}=\mathcal{I}_{K}\left(X,\left\{x_{i}\right\}\right)$ are precisely given as $\mathcal{I}_{K}\left(Y,\left\{x_{i}\right\}\right)$, where $Y$ runs through the ideals of $X$ contained in the principal ideal $\left\langle\leq x_{i}\right\rangle$. Hence the submodule lattice of $P_{i}^{\vee}$ is naturally isomorphic to the lattice of ideals mentioned, where the latter is partially ordered by set-theoretic inclusion. In particular, we have $\operatorname{soc}\left(P_{i}^{\vee}\right) \cong \bigoplus_{j ; x_{j} \leq x_{i} \in X \operatorname{minimal}} T_{j}$ and $\operatorname{rad}\left(P_{i}^{\vee}\right) / \operatorname{rad}^{2}\left(P_{i}^{\vee}\right) \cong$ $\bigoplus_{j ; x_{j} \ll x_{i}} T_{j}$, where $P_{i}^{\vee}$ is simple if and only if $x_{i} \in X$ is minimal.

Then the $K$-dual $I_{i}:=\left(P_{i}^{\vee}\right)^{*}:=\operatorname{Hom}_{K}\left(P_{i}^{\vee}, K\right)$ is an injective $\mathcal{A}$-module, 
which since $\operatorname{soc}\left(I_{i}\right) \cong\left(P_{i}^{\vee} / \operatorname{rad}\left(P_{i}^{\vee}\right)\right)^{*} \cong T_{i}^{*} \cong S_{i}$ is the injective hull of $S_{i}$. The properties of $P_{i}^{\vee}$ by dualising translate into properties of $I_{i}$ : We have $\operatorname{dim}_{K}\left(I_{i}\right)=\operatorname{dim}_{K}\left(P_{i}^{\vee}\right)=\left|\left\langle\leq x_{i}\right\rangle\right|$, where more precisely $\left[I_{i}: S_{j}\right]=1$ if $x_{j} \leq x_{i}$, and $\left[I_{i}: S_{j}\right]=0$ otherwise; in particular, $I_{i}$ is simple if and only if $x_{i} \in X$ is minimal. The quotient modules of $I_{i}$ are given by the ideals of $X$ contained in the principal ideal $\left\langle\leq x_{i}\right\rangle$; in particular, we have $I_{i} / \operatorname{rad}\left(I_{i}\right) \cong \bigoplus_{j ; x_{j} \leq x_{i} \in X \text { minimal }} S_{j}$.

b) In general, injective-indecomposable modules are rather intractable. But this changes for connected components of $X$ having a unique minimal element:

Let $x_{i}$ be the unique minimal element of the connected componens of $X$ under consideration. For $x_{i} \leq x_{j}$ we have $\operatorname{soc}\left(P_{j}^{\vee}\right) \cong T_{i}, \operatorname{implying} I_{j} / \operatorname{rad}\left(I_{j}\right) \cong$ $\left(\operatorname{soc}\left(P_{j}^{\vee}\right)\right)^{*} \cong T_{i}^{*} \cong S_{i}$. Hence $I_{j}$ is an epimorphic image of $P_{i}$. More precisely, since $P_{i} \cong \mathcal{I}_{K}\left(\left\{x_{i}\right\}, X\right)$ is multiplicity-free, and $I_{j}$ has precisely the constituents $S_{k}$ for $x_{k} \leq x_{j}$, this determines $I_{j}$ uniquely as the quotient $I_{j} \cong$ $\mathcal{I}_{K}\left(\left\{x_{i}\right\}, X\right) / \mathcal{I}_{K}\left(\left\{x_{i}\right\}, X \backslash\left\langle\leq x_{j}\right\rangle\right)$; note that $X \backslash\left\langle\leq x_{j}\right\rangle \subseteq X$ is a coideal. $\sharp$

In particular, this applies if $X$ is a lattice: In this case $X$ is connected and has $x_{1}$ as its unique minimal element. Hence all the modules $I_{j}$ occur as epimorphic images of the projective-indecomposable module $P_{1}$.

c) Similarly we are able to determine the indecomposable projective-injective modules: If $P_{i} \cong I_{j}$, then we have $\operatorname{soc}\left(P_{i}\right) \cong S_{j}$, where $x_{j}$ is the unique maximal element such that $x_{i} \leq x_{j}$, and we have $I_{j} / \operatorname{rad}\left(I_{j}\right) \cong S_{i}$, where $x_{i}$ is the unique minimal element such that $x_{i} \leq x_{j}$. Hence the associated connected component of $X$ has $x_{i}$ and $x_{j}$ as its unique minimal and maximal elements, respectively. Conversely, given the latter property, we conclude that $I_{j}$ fulfills $I_{j} / \operatorname{rad}\left(I_{j}\right) \cong S_{i}$, that is $I_{j}$ is an epimorphic image of $P_{i}$, and from $\operatorname{dim}_{K}\left(I_{j}\right)=$ $\left|\left\{k \in\{1, \ldots, n\} ; x_{i} \leq x_{k} \leq x_{j}\right\}\right|=\operatorname{dim}_{K}\left(P_{i}\right)$ we conclude that $I_{j} \cong P_{i}$; note that in this case the constituents $S_{k}$ occurring encompass the full connected component of $X$ under consideration.

In particular, if $X$ is a lattice, in which case $x_{1}$ and $x_{n}$ are the unique minimal and maximal elements, respectively, then the projective-indecomposable module $P_{1}$ is the only projective-injective one, and it coincides with $I_{n}$.

(11.5) The natural module. The natural $\mathcal{T}_{n}(K)$-module $M:=K^{n}$ gives rise to a faithful representation of $\mathcal{A}$, that is for the associated annihilator we have $\operatorname{ann}_{\mathcal{A}}(M)=\{0\}$. Moreover, we have $\operatorname{dim}_{K}\left(\operatorname{Hom}_{\mathcal{A}}\left(E_{i i} \mathcal{A}, M\right)\right)=$ $\operatorname{dim}_{K}\left(M E_{i i}\right)=1$ for all $i \in\{1, \ldots, n\}$, hence we conclude that $\left[M: S_{i}\right]=1$, thus $M$ is multiplicity-free.

Hence the submodule lattice $\mathcal{M}(M)$ of $M$ is described by the set of ideals of $\mathcal{L}(M)$, where for any $i$ there is a unique $S_{i}$-local submodule $L_{i} \leq M$. Since $M E_{i i} \mathcal{A} \leq M$ is a non-zero epimorphic image of $P_{i}=E_{i i} \mathcal{A}$, we conclude that $L_{i}=M E_{i i} \mathcal{A}$. Moreover, we have $L_{i} \leq L_{j}$ if and only if $M E_{i i} \subseteq M E_{j j} \mathcal{A}$, which holds if and only if $M E_{j j} \mathcal{A} E_{i i} \neq\{0\}$, which in turn since $M$ is faithful holds if and only if $\operatorname{Hom}_{\mathcal{A}}\left(P_{i}, P_{j}\right)=\operatorname{Hom}_{\mathcal{A}}\left(E_{i i} \mathcal{A}, E_{j j} \mathcal{A}\right) \cong E_{j j} \mathcal{A} E_{i i} \neq\{0\}$, where the latter is equivalent to $x_{j} \leq x_{i}$. 
Thus as partially ordered sets $\mathcal{L}(M)$ is the dual of $X$, and the ideals of $\mathcal{L}(M)$ are in bijection with the coideals of $X$. The block components of $M$ are indecomposable, coinciding with the lattice block components, and are given by the connected components of $X$; thus $M$ is indecomposable if and only if $X$ is connected. To describe when a block components of $M$ is projective or injective, we may assume that $X$ is connected: Then $M$ is a projective-indecomposable module if and only if $M \cong P_{1}$, which holds if and only if $x_{1}$ is the unique minimal element of $X$; similarly, $M$ is an injective-indecomposable module if and only if $M \cong I_{n}$, which holds if and only if $x_{n}$ is the unique maximal element.

(11.6) Characterizing incidence algebras. We proceed to give a representation theoretic characterization of finite dimensional incidence algebras; this is inspired by [16]. The starting point is the above observation that all local submodules of all the projective-indecomposable $\mathcal{A}$-modules $P_{i}$ are projective again, that is to say that $\mathcal{A}$ is locally hereditary [Bautista, 1981]. We first discuss a few general related properties:

a) Any locally hereditary basic finite-dimensional $K$-algebra $\mathcal{A}$ necessarily has an acyclic Ext quiver: Any arrow $T \leftarrow S$ in the Ext quiver, where $S$ and $T$ are simple $\mathcal{A}$-modules, gives rise to an embedding $P_{T} \rightarrow P_{S}$ of the associated projective covers; hence a putative oriented cycle in the Ext quiver through $S$ would entail a non-surjective embedding $P_{S} \rightarrow P_{S}$, a contradiction.

Moreover, acyclicity of the Ext quiver entails $\left[P_{S}: S\right]=1$ for all simple $\mathcal{A}$ module $S$ : Assume to the contrary that $\left[P_{S}: S\right] \geq 2$, then there is a $k$-fold extension $\{0\} \rightarrow S \rightarrow V_{k} \rightarrow \cdots \rightarrow V_{1} \rightarrow S \rightarrow\{0\}$, for some $k \in \mathbb{N}$, where the $V_{l}$ are indecomposable $\mathcal{A}$-modules of composition length 2 ; hence there is a cycle $S \leftarrow \cdots \leftarrow S$ of length $k$ in the Ext quiver, a contradiction.

b) A finite-dimensional $K$-algebra $\mathcal{A}$ is called hereditary if all its right ideals are projective. Then $\mathcal{A}$ is hereditary if and only if its Jacobson radical is a projective module, which holds if and only if any submodule of any projectiveindecomposable module is projective again. In particular, hereditary algebras are indeed locally hereditary, but the converse does not hold.

Hence, if $\mathcal{A}$ is an incidence algebra, then the discussion in (11.3) shows that it is hereditary if and only if all connected components of the underlying partially ordered set $X$ are trees.

(11.7) Theorem: Iovanov-Koffi [2017]. Let $\mathcal{A}$ be a basic finite-dimensional $K$-algebra. Then the following are are equivalent:

i) $\mathcal{A}$ is an incidence algebra.

ii) $\mathcal{A}$ has a faithful multiplicity-free representation.

iii) $\mathcal{A}$ has a faithful representation with distributive submodule lattice, and we have $\left[P_{S}: S\right]=1$ for all simple $\mathcal{A}$-modules $S$.

Proof. We have already seen that the natural module of an incidence algebra fulfills the properties of ii), hence the implication 'i) $\Rightarrow$ ii)' holds. 
a) We show the equivalence 'ii) $\Leftrightarrow$ iii)' without reference to incidence algebras. To this end, let $M$ be a faithful $\mathcal{A}$-module. Since for any primitive idempotent $e \in \mathcal{A}$ we have $M e \neq\{0\}$, we infer that $[M: S] \neq 0$ for all simple $\mathcal{A}$-modules $S$.

Let now $M$ be multiplicity-free, that is $[M: S]=1$ for all simple $\mathcal{A}$-modules $S$, which implies that $\mathcal{M}(M)$ is distributive. In order to show the second statement, let $S$ be any simple $\mathcal{A}$-module, and let $e \in \mathcal{A}$ be a primitive idempotent associated with $S$. Then we have $\operatorname{dim}_{K}\left(\operatorname{Hom}_{\mathcal{A}}(e A, M)\right)=\operatorname{dim}_{K}(M e)=1$, and letting $0 \neq \varphi \in \operatorname{Hom}_{\mathcal{A}}(e A, M)$ we have $M e=\langle\varphi(e)\rangle_{K}$, and hence $M \cong$ $M(1-e) \oplus\langle\varphi(e)\rangle_{K}$. For $e a \in \operatorname{ker}(\varphi)$ we have $\varphi(e) \cdot e a=\varphi(e a)=0$, and since $M(1-e) \cdot e a=\{0\}$ anyway, we infer that $e a \in \operatorname{ann}_{A}(M)=\{0\}$. Thus $\varphi$ is injective, implying that $e A \cong \varphi(e A) \leq M$ is multiplicity-free, in particular we have $\left[P_{S}: S\right]=1$. This shows the implication 'ii) $\Rightarrow$ iii)'.

Conversely, let $\mathcal{M}(M)$ be distributive. Letting $S$ be a simple $\mathcal{A}$-module, $\mathcal{L}_{S}(M)$ is non-empty and a chain. Assume that $\left|\mathcal{L}_{S}(M)\right| \geq 2$, then there is an $S$-local submodule $L \leq M$ such that $[L: S] \geq 2$, and $L$ being an epimorphic image of $P_{S}$, we infer $\left[P_{S}: S\right] \geq 2$, a contradiction. Hence $\mathcal{L}_{S}(M)$ is a singleton set, that is $[M: S]=1$. Thus $M$ is multiplicity-free, showing the implication 'iii) $\Rightarrow$ ii)'.

b) To show the implication 'ii) $\Rightarrow \mathrm{i}$ )', let $M$ be a faithful multiplicity-free $\mathcal{A}$ module, such that $n:=\operatorname{dim}_{K}(M) \in \mathbb{N}$. Hence let $\left\{S_{1}, \ldots, S_{n}\right\}$ be the simple $\mathcal{A}$-modules, up to isomorphism, and let $1=\sum_{i=1}^{n} e_{i} \in \mathcal{A}$ be a decomposition into pairwise orthogonal primitive idempotents, where we assume notation chosen such that $e_{i}$ acts as the identity on $S_{i}$.

As we have seen in the proof of 'ii) $\Rightarrow$ iii)' above, $e_{i} \mathcal{A}$ is multiplicity-free, hence we have $\operatorname{dim}_{K}\left(e_{i} \mathcal{A} e_{j}\right)=\operatorname{dim}_{K}\left(\operatorname{Hom}_{\mathcal{A}}\left(e_{j} \mathcal{A}, e_{i} \mathcal{A}\right)\right)=\left[e_{i} \mathcal{A}: S_{j}\right] \leq 1$ for all $i, j \in$ $\{1, \ldots, n\}$. Whenever $\left[e_{i} \mathcal{A}: S_{j}\right]=1$, we let $0 \neq \varphi_{i j} \in \operatorname{Hom}_{\mathcal{A}}\left(e_{j} \mathcal{A}, e_{i} \mathcal{A}\right)$ and $e_{i j}:=\varphi_{i j}\left(e_{j}\right) \in e_{i} \mathcal{A} e_{j}$; for $i=j$ we may assume that $\varphi_{i i}=\operatorname{id}_{e_{i} \mathcal{A}}$, that is $e_{i i}=e_{i}$, while $\varphi_{i j}$ is not surjective whenever $i \neq j$. Hence we have the Pierce decomposition $\mathcal{A} \cong \bigoplus_{\left[e_{i} \mathcal{A}: S_{j}\right]=1} e_{i} \mathcal{A} e_{j}=\bigoplus_{\left[e_{i} \mathcal{A}: S_{j}\right]=1}\left\langle e_{i j}\right\rangle_{K}$ as $K$ vector spaces.

Moreover, any non-zero homomorphism $e_{i} \mathcal{A} \rightarrow M$ is injective. Hence choosing $0 \neq \varphi_{i} \in \operatorname{Hom}_{\mathcal{A}}\left(e_{i} \mathcal{A}, M\right)$, from $\varphi_{i j} \neq 0$ we infer that $\varphi_{i} \varphi_{i j} \in \operatorname{Hom}_{\mathcal{A}}\left(e_{j} \mathcal{A}, M\right)$ is non-zero, and hence injective, implying that $\varphi_{i j}$ is injective as well. Hence, whenever $\left[e_{i} \mathcal{A}: S_{j}\right]=1=\left[e_{j} \mathcal{A}: S_{k}\right]$, we have $\varphi_{i j} \varphi_{j k} \neq 0$, implying that $\left[e_{i} \mathcal{A}: S_{k}\right]=1$ as well, and there is $a_{i j k} \in K^{*}$ such that $\varphi_{i j} \varphi_{j k}=a_{i j k} \varphi_{i k}$; in particular we have $a_{i j j}=a_{j j k}=a_{i i i}=1$. Moreover, for $k=i$ from $\varphi_{i j} \varphi_{j i} \neq 0$ we infer that this is only possible for $j=i$.

Letting $X:=\left\{x_{1}, \ldots, x_{n}\right\}$, we may define a relation $\leq$ on $X$ by $x_{i} \leq x_{j}$ if and only if $\left[e_{i} \mathcal{A}: S_{j}\right]=1$. By the above considerations this relation is reflexive, antisymmetric and transitive, that is a partial order. We show that $\mathcal{A} \cong \mathcal{A}_{K}(X)$ : We have already seen that $\mathcal{A} \cong \bigoplus_{x_{i}<x_{j}}\left\langle e_{i j}\right\rangle_{K}$, where multiplication is given by $e_{i j} e_{j k}=a_{i j k} e_{i k}$, whenever $x_{i} \leq x_{j} \leq x_{k}$. In other words, multiplication in $\mathcal{A}$ is 'twisted' compared to $\mathcal{A}_{K}(X)$, but in a trivial sense as follows:

Recalling that $M e_{i}=\left\langle\varphi_{i}\left(e_{i}\right)\right\rangle_{K}$, we get $\varphi_{i}\left(e_{i}\right) \cdot e_{i j}=\varphi_{i}\left(e_{i j}\right)=\varphi_{i}\left(e_{i j}\right) \cdot e_{j}=$ 
$b_{i j} \cdot \varphi_{j}\left(e_{j}\right) \in M$, for suitable $b_{i j} \in K^{*}$; note that $b_{i i}=1$. This yields $\varphi_{i}\left(e_{i}\right) \cdot$ $e_{i j} \cdot e_{j k}=b_{i j} \cdot \varphi_{j}\left(e_{j}\right) \cdot e_{j k}=b_{i j} b_{j k} \cdot \varphi_{k}\left(e_{k}\right) \in M$ and $\varphi_{i}\left(e_{i}\right) \cdot e_{i j} e_{j k}=a_{i j k}$. $\varphi_{i}\left(e_{i}\right) \cdot e_{i k}=a_{i j k} b_{i k} \cdot \varphi_{k}\left(e_{k}\right) \in M$, hence equating yields $a_{i j k}=\frac{b_{i j} b_{j k}}{b_{i k}}$. Thus let $e_{i j}^{\prime}:=\frac{1}{b_{i j}} \cdot e_{i j} \in \mathcal{A}$, for all $i, j \in\{1, \ldots, n\}$ such that $x_{i} \leq x_{j}$; note that $e_{i i}^{\prime}=e_{i i}$. Then we get $\mathcal{A} \cong \bigoplus_{x_{i} \leq x_{j}}\left\langle e_{i j}^{\prime}\right\rangle_{K}$, such that $e_{i j}^{\prime} e_{j k}^{\prime}=\frac{1}{b_{i j} b_{j k}} \cdot a_{i j k} \cdot\left(b_{i k} \cdot e_{i k}^{\prime}\right)=e_{i k}^{\prime}$. This proves $\mathcal{A} \cong \mathcal{A}_{K}(X)$, and hence the implication 'ii) $\Rightarrow$ i)' holds.

\section{Generating functions}

\section{Power series}

(12.1) Formal power series. a) Let $K$ be a field. We consider the $K$-vector space $K[[X]]:=\operatorname{Maps}\left(\mathbb{N}_{0}, K\right)$, with pointwise addition and scalar multiplication. We write the elements of $K[[X]]$, that is sequences $\left[f_{n} \in K ; n \in \mathbb{N}_{0}\right]$, as (ordinary) generating series or formal power series $f:=\sum_{n>0} f_{n} X^{n}$, where $X$ is an indeterminate. The principle of comparison of coefficients holds, saying that $f, g \in K[[X]]$ are equal if and only if $f_{n}=g_{n}$ for all $n \in \mathbb{N}_{0}$.

Then $K[[X]]$ is a commutative $K$-algebra with respect to convolutional multiplication $\left(\sum_{i \geq 0} f_{i} X^{i}\right) \cdot\left(\sum_{j \geq 0} g_{j} X^{j}\right):=\sum_{n \geq 0} \sum_{k=0}^{n}\left(f_{k} g_{n-k}\right) X^{n} \in K[[X]]$, the neutral element being $1:=X^{0} \in K[[X]]$; note that to determine any fixed coefficient only finitely many arithmetical operations in $K$ are necessary, and that the polynomial ring $K[X] \subseteq K[[X]]$ is a subring.

Let $\nu(f)=\nu_{X}(f):=\min \left\{n \in \mathbb{N}_{0} ; f_{n} \neq 0\right\} \in \mathbb{N}_{0}$, for any $0 \neq f \in K[[X]]$, be the order or (discrete) valuation of $f$ at $X$; for completeness we let $\nu(0)=\infty$. Then for any $0 \neq f, g \in K[[X]]$, letting $n:=\nu(f) \in \mathbb{N}_{0}$ and $m:=\nu(g) \in \mathbb{N}_{0}$, we have $f g=f_{n} g_{m} X^{n+m}+\sum_{k>n+m+1} h_{k} X^{k} \in K[[X]]$, for suitable $h_{k} \in K$, implying that $\nu(f g)=n+m \in \overline{\mathbb{N}}_{0}$. In particular, we have $f g \neq 0 \in K[[X]]$, implying that $K[[X]]$ is an integral domain.

Moreover, $\nu_{X}:(K[[X]] \backslash\{0\}, \cdot) \rightarrow(\mathbb{Z},+)$ is a monoid homomorphism into a totally ordered abelian group. The set $\mathcal{I}_{n}:=\{f \in K[[X]] ; \nu(f) \geq n\}=$ $X^{n} K[[X]] \unlhd K[[X]]$ is an ideal, for all $n \in \mathbb{N}_{0}$. We have $K[[X]]=\mathcal{I}_{0} \supset \mathcal{I}_{1} \supset \cdots$, where $\bigcap_{n \in \mathbb{N}_{0}} \mathcal{I}_{n}=\{0\}$, and $K[[X]] / \mathcal{I}_{1} \cong K$ as $K$-algebras, and $\mathcal{I}_{n+1} / \mathcal{I}_{n} \cong K$ as $K$-vector spaces, for all $n \in \mathbb{N}$. In particular, we conclude that $K[[X]]$ is a complete discrete valuation ring.

Let $K[[X]]^{*} \subseteq K[[X]]$ be the set of all invertible elements. Then we have $K[[X]]^{*}=K[[X]] \backslash X K[[X]]=\left\{f \in K[[X]] ; f_{0} \neq 0\right\}=\{f \in K[[X]] ; \nu(f)=0\}:$ If $f \in K[[X]]$ has inverse $g \in K[[X]]$, then from $f g=1 \in K[[X]]$ we get $f_{0} g_{0}=$ $1 \in K$, thus $f_{0} \neq 0$; conversely, if $f \in K[[X]]$ such that $f_{0} \neq 0$, then letting $g_{0}:=$ $f_{0}^{-1} \in K$, and for $n \in \mathbb{N}$ by induction letting $g_{n}:=-f_{0}^{-1} \cdot \sum_{k=0}^{n-1} f_{n-k} g_{k} \in K$, for $g:=\sum_{n \geq 0} g_{n} X^{n} \in K[[X]]$ we get $f g=\sum_{n \geq 0}\left(\sum_{k=0}^{n} f_{n-k} g_{k}\right) X^{n}=1 \in K[[X]]$, hence $g$ is the inverse of $f$. 
The field of fractions $K((X))$ of $K[[X]]$ can be identified with the set of formal Laurent series $f:=\sum_{n \geq m} f_{n} X^{n}$, where $m \in \mathbb{Z}$ and $f_{n} \in K$, for all $n \geq$ $m$ : Indeed, convolutional multiplication extends to $K((X))$, and the valuation extends to $K((X))$ by letting $\nu(f):=\min \left\{n \in \mathbb{Z} ; f_{n} \neq 0\right\} \in \mathbb{Z}$, for $0 \neq f \in$ $K((X))$. Then for $0 \neq f \in K((X))$ we have $X^{-\nu(f)} f \in K[[X]]^{*}$, and hence letting $g:=\left(X^{-\nu(f)} f\right)^{-1} \in K[[X]]^{*}$ we get $f^{-1}=X^{-\nu(f)} g \in K((X))$. Note that the field $K(X)$ of rational functions, that is the field of fractions of $K[X]$, is a subfield of $K((X))$.

b) Then for all $f \in X K[[X]]$, that is $f_{0}=0$, we have $\nu(f) \geq 1$, and hence $\nu\left(f^{n}\right)=n \nu(f) \geq n$, for all $n \in \mathbb{N}_{0}$. Thus for all $g \in K[[X]]$ and $f \in X K[[X]]$, the composition $g(f):=\sum_{n \geq 0} g_{n} f(X)^{n} \in K[[X]]$ is well-defined.

Similarly, given a sequence $\left[F_{i} \in K[[X]] ; i \in \mathbb{N}\right]$ such that $\lim _{i \rightarrow \infty} \nu\left(F_{i}\right)=$ $\infty$, the infinite sum $\sum_{i>1} F_{i} \in K[[X]]$ is well-defined; and given a sequence $\left[1+F_{j} \in 1+X K[[X]] ; j \in \mathbb{N}\right]$ such that $\lim _{j \rightarrow \infty} \nu\left(F_{j}\right)=\infty$, the infinite product $\prod_{j \geq 1}\left(1+F_{j}\right) \in K[[X]]$ is well-defined.

The formal derivative defined as $\frac{\partial}{\partial X}: K[[X]] \rightarrow K[[X]]: \sum_{n \geq 0} f_{n} X^{n} \mapsto$ $\sum_{n \geq 1} n f_{n} X^{n-1}=\sum_{n \geq 0}(n+1) f_{n+1} X^{n}$ is $K$-linear, and we have the product rule $\frac{\partial}{\partial X}(f g)=\left(\frac{\partial}{\partial X} f\right) g+f\left(\frac{\partial}{\partial X} g\right) \in K[[X]]$, for $f, g \in K[[X]]$, and the chain rule $\frac{\partial}{\partial X}(g(f))=\left(\frac{\partial}{\partial X} g\right)(f) \cdot\left(\frac{\partial}{\partial X} f\right) \in K[[X]]$, for $g \in K[[X]]$ and $f \in X K[[X]]$ :

By $K$-linearity, for the product rule it suffices to note that $\frac{\partial}{\partial X}\left(X^{m} X^{n}\right)=(m+$ $n) X^{m+n-1}=m X^{m-1} X^{n}+n X^{m} X^{n-1}=\frac{\partial}{\partial X}\left(X^{m}\right) \cdot X^{n}+X^{m} \cdot \frac{\partial}{\partial X}\left(X^{n}\right) \in K[[X]]$, for $m, n \in \mathbb{N}$. Similarly, for the chain rule, it suffices to show, by induction on $n \in \mathbb{N}$, that $\frac{\partial}{\partial X}\left(f^{n}\right)=n f^{n-1} \cdot \frac{\partial}{\partial X}(f) \in K[[X]]$, for $f \in X K[[X]]$ : The case $n=1$ being trivial, let $n \geq 2$. Then by induction and using the product rule we have $\frac{\partial}{\partial X}\left(f^{n}\right)=\frac{\partial}{\partial X}\left(f^{n-1} f\right)=\frac{\partial}{\partial X}\left(f^{n-1}\right) \cdot f+f^{n-1} \cdot \frac{\partial}{\partial X}(f)=n f^{n-1} \cdot \frac{\partial}{\partial X}(f)$. \#

(12.2) Taylor series. a) For $K=\mathbb{C}$ this is related to Taylor series expansions around $x=0$, generalizing the connection between polynomials and polynomial maps: Let $\mathbb{C}[[X]]^{\infty} \subseteq \mathbb{C}[[X]]$ be the $\mathbb{C}$-subalgebra of formal power series $f=$ $\sum_{n \geq 0} f_{n} X^{n} \in \mathbb{C}[[X]]$ such that the associated Taylor series $\widehat{f}: \mathcal{O} \rightarrow \mathbb{C}: x \mapsto$ $\sum_{n \geq 0} f_{n} x^{n}$ converges on an open disc $\mathcal{O} \subseteq \mathbb{C}$ of positive radius centered at $x=0$. Hence, letting $\mathcal{H}$ be the $\mathbb{C}$-algebra of holomorphic function germs around $x=0$, by the principle of comparison of coefficients the map $\mathbb{C}[[X]]^{\infty} \rightarrow$ $\mathcal{H}: f \mapsto \widehat{f}$ is an isomorphism of $\mathbb{C}$-algebras. Moreover, for $f \in \mathbb{C}[[X]]^{\infty}$ the valuation $\nu(f) \in \mathbb{N}_{0}$ coincides with the order of $x=0$ as a zero of $\widehat{f}$.

b) For example, let the exponential series be defined as $\exp :=\sum_{n \geq 0} \frac{1}{n !} X^{n} \in$ $1+X \mathbb{Q}[[X]] \subseteq \mathbb{Q}[[X]]$. Then for the associated Taylor series we have $\exp (x)=$ $\exp (x)$, for all $x \in \mathbb{C}$, hence we have $\exp \in \mathbb{C}[[X]]^{\infty}$. Moreover, we have $\frac{\partial}{\partial X} \exp =\sum_{n \geq 1} \frac{1}{(n-1) !} X^{n-1}=\sum_{n \geq 0} \frac{1}{n !} X^{n}=\exp \in \mathbb{Q}[[X]]$.

If $K$ is a field of characteristic 0 , then for $f \in X K[[X]]$ we have $\exp (f):=$ $\sum_{n \geq 0} \frac{1}{n !} f^{n} \in 1+X K[[X]] \subseteq K[[X]]$, fulfilling the identity $\exp (f+g)=$ 
$\sum_{n \geq 0} \frac{1}{n !}(f+g)^{n}=\sum_{n \geq 0} \sum_{k=0}^{n} \frac{1}{k !(n-k) !} f^{k} g^{n-k}=\left(\sum_{i \geq 0} \frac{1}{i !} f^{i}\right) \cdot\left(\sum_{j \geq 0} \frac{1}{j !} g^{j}\right)=$ $\exp (f) \cdot \exp (g) \in K[[X]]$, for all $f, g \in X K[[X]]$, hence $\exp (f)^{-1}=\exp (-f) \in$ $K[[X]]$. In particular, we have $\exp (X) \cdot \exp (-X)=1 \in \mathbb{Q}[[X]]$, from which we recover the formula $\sum_{k=0}^{n} \frac{(-1)^{n-k}}{k !(n-k) !}=\delta_{0, n}$, that is $\sum_{k=0}^{n}(-1)^{n-k}\left(\begin{array}{c}n \\ k\end{array}\right)=\delta_{0, n}$, for $n \in \mathbb{N}_{0}$. Note that going over to the associated Taylor series yields the identity $\exp (x) \cdot \exp (-x)=1 \in \mathbb{C}$, for all $x \in \mathbb{C}$; but conversely the identity $\exp (x+1)=e \cdot \exp (x) \in \mathbb{C}$, yielding $\sum_{n>0} \frac{1}{n !}(x+1)^{n}=e \cdot \sum_{m>0} \frac{1}{m !} x^{m}$ for all $x \in \mathbb{C}$, cannot be translated to the formal setting.

Let $\log :=\sum_{n \geq 1} \frac{(-1)^{n-1}}{n} X^{n} \in X \mathbb{Q}[[X]] \triangleleft \mathbb{Q}[[X]]$ be the logarithm series. Then for the associated Taylor series we have $\widehat{\log }(x)=\sum_{n \geq 1} \frac{(-1)^{n-1}}{n} x^{n}=$ $\ln (x+1)$, for all $x \in \mathbb{C}$ such that $|x|<1$, hence $\log \in \mathbb{C}[[X]]^{\infty}$. Moreover, we have $\frac{\partial}{\partial X} \log =\sum_{n \geq 1}(-1)^{n-1} X^{n-1}=\frac{1}{1+X} \in \mathbb{Q}[[X]]$, and going over to the associated Taylor series we get $\exp (\log )=1+X \in \mathbb{Q}[[X]]$ and $\log (\exp -1)=X \in \mathbb{Q}[[X]]$.

If $K$ is a field of characteristic 0 , then for $f \in X K[[X]]$ we have $\log (f):=$ $\sum_{n \geq 0} \frac{(-1)^{n-1}}{n} f^{n} \in 1+X K[[X]] \subseteq K[[X]]$, fulfilling the identity $\log ((f+1)(g+$ $1)-1)=\log (f)+\log (g) \in K[[X]]$, for all $f, g \in X K[[X]]:$ We have $\exp (\log ((f+$ 1) $(g+1)-1))=(f+1)(g+1)=\exp (\log (f)) \cdot \exp (\log (g))=\exp (\log (f)+\log (g)) \in$ $K[[X]]$, thus $\log ((f+1)(g+1)-1)=\log (\exp (\log ((f+1)(g+1)-1))-1)=$ $\log (\exp (\log (f)+\log (g))-1)=\log (f)+\log (g) \in K[[X]]$. Note that this for $f, g \in 1+X K[[X]]$ yields $\log (f g-1)=\log (f-1)+\log (g-1) \in K[[X]]$.

(12.3) Binomial series. a) The simplest generating series are the polynomials, being associated to finite sequences. For example, for $n \in \mathbb{N}_{0}$, for the sequence of binomial coefficients we have $\sum_{k=0}^{n}\left(\begin{array}{l}n \\ k\end{array}\right) X^{k}=(1+X)^{n} \in \mathbb{Z}[X]$, and for the Stirling numbers of the first kind we have $\sum_{k=0}^{n} s_{n, k} X^{k}=X^{(n)} \in \mathbb{Z}[X]$.

For the sequence of Stirling numbers of the second kind we similarly have $X^{n}=$ $\sum_{k=0}^{n} S_{n, k} X_{(k)} \in \mathbb{Z}[X]$, which does not translate into a simple formula for the associated generating series; but recalling that both $\left\{X^{k} ; k \in\{0, \ldots, n\}\right\}$ and $\left\{X_{(k)} ; k \in\{0, \ldots, n\}\right\}$ are $\mathbb{Z}$-bases of $\mathbb{Z}[X]_{\leq n}$ shows that this differs from a proper generating series only by a base change.

The other way around, fixing $k \in \mathbb{N}_{0}$, for the generating series of binomial coefficients we get $\sum_{n \geq 0}\left(\begin{array}{l}n \\ k\end{array}\right) X^{n}=\frac{X^{k}}{k !} \cdot \sum_{n \geq k} n_{(k)} X^{n-k}=\frac{X^{k}}{k !} \cdot\left(\frac{\partial}{\partial X}\right)^{k}\left(\sum_{n \geq 0} X^{n}\right)=$ $\frac{X^{k}}{k !} \cdot\left(\frac{\partial}{\partial X}\right)^{k}\left(\frac{1}{1-X}\right)=\frac{X^{k}}{(1-X)^{k+1}} \in \mathbb{Q}[[X]]$.

b) We proceed towards a generalization of the generating series of binomial coefficients: Let $K$ be a field. Then we have $1-X \in K[X] \cap K[[X]]^{*}$ such that $(1-X)^{-1}=\frac{1}{1-X}=\sum_{n>0} X^{n} \in K(X) \cap K[[X]]$. Using the combinatorial reciprocity $\left|\mathcal{M}_{k}(n)\right|=\left(\begin{array}{c}k+n-1 \\ k\end{array}\right)=(-1)^{k} \cdot\left(\begin{array}{c}-n \\ k\end{array}\right)$, for $n \in \mathbb{N}$, this yields the binomial series $\frac{1}{(1-X)^{n}}=\sum_{k \geq 0}\left|\mathcal{M}_{k}(n)\right| \cdot X^{k}=\sum_{k \geq 0}\left(\begin{array}{c}-n \\ k\end{array}\right) \cdot(-X)^{k} \in K[[X]]$. Thus for all $n \in \mathbb{Z}$ we have $(1+X)^{n}=\sum_{k \geq 0}\left(\begin{array}{l}n \\ k\end{array}\right) X^{k} \in K[[X]]$, being a polynomial if and only if $n \geq 0$. In particular, the generating series of binomial coefficients considered above equals the binomial series $\sum_{n \geq 0}\left(\begin{array}{l}n \\ k\end{array}\right) X^{n}=\frac{X^{k}}{(1-X)^{k+1}}=$ 
$\sum_{n \geq 0}(-1)^{n}\left(\begin{array}{c}-k-1 \\ n\end{array}\right) X^{n+k} \in \mathbb{Q}[[X]]$, for $k \in \mathbb{N}_{0}$.

Let $K$ be a field of characteristic 0 . Letting $Z$ be an indeterminate, we let $(1+X)^{Z}:=\sum_{k>0}\left(\begin{array}{l}Z \\ k\end{array}\right) X^{k} \in K[Z][[X]]$. Thus by evaluating for $z \in K$ we let $(1+X)^{z}:=\sum_{k>0}\left(\begin{array}{l}z \\ k\end{array}\right) X^{k} \in K[[X]] ;$ in particular for $n \in \mathbb{Z}$ we recover the above expression for $(1+X)^{n}$. Then, letting $Y$ be an indeterminate, the Vandermonde identity implies $(1+X)^{Y} \cdot(1+X)^{Z}=\left(\sum_{i \geq 0}\left(\begin{array}{c}Y \\ i\end{array}\right) X^{i}\right) \cdot\left(\sum_{j \geq 0}\left(\begin{array}{c}Z \\ j\end{array}\right) X^{j}\right)=$ $\sum_{n \geq 0}\left(\sum_{k=0}^{n}\left(\begin{array}{c}Y \\ k\end{array}\right)\left(\begin{array}{c}Z \\ n-k\end{array}\right)\right) X^{n}=\sum_{n \geq 0}\left(\begin{array}{c}Y+Z \\ n\end{array}\right) X^{n}=(1+X)^{Y+Z} \in K[Y, Z][[X]] ;$ thus for all $y, z \in K$ we get $(1+\bar{X})^{y} \cdot(1+X)^{z}=(1+X)^{y+z} \in K[[X]]$. Note that for $K:=\mathbb{C}$ we have $(1+X)^{z} \in \mathbb{C}[[X]]^{\infty}$ with Taylor series $(1+x)^{z}=$ $\sum_{k \geq 0}\left(\begin{array}{l}z \\ k\end{array}\right) x^{k} \in \mathbb{C}$, for all $x \in \mathbb{C}$ such that $|x|<1$.

c) As an application of the above machinery we consider the infinite product $f:=\prod_{n \geq 1}\left(1-X^{n}\right)^{\frac{-\mu(n)}{n}} \in \mathbb{Q}[[X]]$, where $\mu: \mathbb{N} \rightarrow \mathbb{N}$ is the number theoretic Möbius function; since $\nu\left(\left(1-X^{n}\right)^{\frac{-\mu(n)}{n}}-1\right)=\nu\left(\sum_{k>1}\left(\frac{-\mu(n)}{k}\right) \cdot(-1)^{k} X^{k n}\right)=n$, for $n \in \mathbb{N}$, the infinite product is well-defined. Then we get $\log (f-1)=$ $-\sum_{n \geq 1} \frac{\mu(n)}{n} \cdot \log \left(-X^{n}\right)=\sum_{n \geq 1}\left(\frac{\mu(n)}{n} \cdot \sum_{k \geq 1} \frac{X^{k n}}{k}\right)=\sum_{n \geq 1}\left(\sum_{d \mid n} \mu(d)\right) \cdot \frac{X^{n}}{n}=$ $\sum_{n \geq 1} \delta_{1, n} \cdot \frac{X^{n}}{n}=X \in \mathbb{Q}[[X]]$, hence $\exp =\exp (\log (f-1))=f \in \mathbb{Q}[[X]]$.

(12.4) Rational functions. a) Let $d \in \mathbb{N}$ and $q_{1}, \ldots, q_{d} \in \mathbb{C}$ such that $q_{d} \neq 0$, let $q=1+\sum_{i=1}^{d} q_{i} X^{i}=\prod_{j=1}^{k}\left(1-a_{j} X\right)^{d_{j}} \in \mathbb{C}[X] \cap \mathbb{C}[[X]]^{*}$, for some $k \in \mathbb{N}$ and $d_{j} \in \mathbb{N}$, and pairwise distinct $0 \neq a_{1}, \ldots, a_{k} \in \mathbb{C}$; hence we have $\sum_{j=1}^{k} d_{j}=d$. Letting $f:=\sum_{n \geq 0} f_{n} X^{n} \in \mathbb{C}[[X]]$, the following are equivalent:

i) The series $f$ is rational $f=\frac{p}{q} \in \mathbb{C}(X) \cap \mathbb{C}[[X]]$, where $p \in \mathbb{C}[X]_{\leq d-1}$.

ii) We have the partial fraction decomposition $f=\sum_{j=1}^{k} \frac{g_{j}}{\left(1-a_{j} X\right)^{d_{j}}} \in$ $\mathbb{C}(X) \cap \mathbb{C}[[X]]$, where $g_{j} \in \mathbb{C}[X]_{\leq d_{j}-1}$ for all $j \in\{1, \ldots, k\}$.

iii) The sequence $\left[f_{n} \in \mathbb{C} ; n \in \mathbb{N}_{0}\right]$ is a linear recurrent sequence of degree $d$, that is $f_{n+d}+\sum_{i=1}^{d} q_{i} f_{n+d-i}=0$ for all $n \in \mathbb{N}_{0}$.

iv) We have $f_{n}=\sum_{j=1}^{k} h_{j}(n) a_{j}^{n}$, for all $n \in \mathbb{N}_{0}$, where $h_{j} \in \mathbb{C}[X]_{\leq d_{j}-1}$.

In order to see this let $V_{(i)}, V_{(i i)}, V_{(i i i)}, V_{(i v)} \leq \mathbb{C}[[X]]$ be the $\mathbb{C}$-subspaces of all formal power series fulfilling property (i), (ii), (iii) and (iv), respectively. We show that these $\mathbb{C}$-subspaces actually coincide:

For $f \in V_{(i i)}$, letting $q_{j}:=\left(1-a_{j} X\right)^{d_{j}} \in \mathbb{C}[X]$ and $r_{j}:=\frac{q}{q_{j}} \in \mathbb{C}[X]$, for $j \in\{1, \ldots, k\}$, yields $f=\frac{1}{q} \cdot \sum_{j=1}^{k} g_{j} r_{j} \in \mathbb{C}[[X]]$, where $g_{j}=0$ or $\operatorname{deg}\left(g_{j}\right)+$ $\operatorname{deg}\left(r_{j}\right)<d_{j}+\left(d-d_{j}\right)=d$, showing that $V_{(i i)} \leq V_{(i)}$. Moreover, recalling that $\mathbb{C}[X]$ is an Euclidean domain, let $s_{j} \in \mathbb{C}[X]$ such that $r_{j} s_{j}=1 \in \mathbb{C}[X] / q_{j} \mathbb{C}[X]$; note that in particular $s_{j} \in \mathbb{C}[X] / q_{j} \mathbb{C}[X]$ is invertible. Then, since the $q_{j} \in \mathbb{C}[X]$ are pairwise coprime, the Chinese remainder theorem shows that the map $\bigoplus_{j=1}^{k} \mathbb{C}[X] / q_{j} \mathbb{C}[X] \rightarrow \mathbb{C}[X] / q \mathbb{C}[X]:\left[g_{1}, \ldots, g_{k}\right] \rightarrow \sum_{j=1}^{k} g_{j} s_{j} r_{j}$ is an isomorphism of $\mathbb{C}$-algebras, thus $\bigoplus_{j=1}^{k} \mathbb{C}[X] / q_{j} \mathbb{C}[X] \rightarrow \mathbb{C}[X] / q \mathbb{C}[X]:\left[g_{1}, \ldots, g_{k}\right] \rightarrow$ $\sum_{j=1}^{k} g_{j} r_{j}$ is as well, in particular is injective. Hence choosing the coefficients of 
the $g_{j} \in \mathbb{C}[X]_{\leq d_{j}-1}$, for $j \in\{1, \ldots, k\}$, shows that $\operatorname{dim}_{\mathbb{C}}\left(V_{(i i)}\right)=\sum_{j=1}^{k} d_{j}=d$. Similarly, choosing the coefficients of $p \in \mathbb{C}[X]_{\leq d-1}$ shows that $\operatorname{dim}_{\mathbb{C}}\left(V_{(i)}\right)=d$, thus we conclude that $V_{(i)}=V_{(i i)}$. Note that, as an alternative, for $f \in V_{(i)} \cup V_{(i i)}$ we may consider the associated Taylor series, which converges for all $x \in \mathbb{C}$ such that $|x|<\frac{1}{\left|\alpha_{j}\right|}$, for all $j \in\{1, \ldots, k\}$, hence $V_{(i)}=V_{(i i)}$ also follows from considering the partial fraction decomposition of rational maps.

Since $f \in V_{(i i i)}$ is uniquely determined by the initial sequence $\left[f_{0}, \ldots, f_{d-1}\right]$, we have $\operatorname{dim}_{\mathbb{C}}\left(V_{(i i i)}\right) \leq d$. For $f \in V_{(i)}$, from $\left(\sum_{n \geq 0} f_{n} X^{n}\right)\left(1+\sum_{i=1}^{d} q_{i} X^{i}\right)=$ $f q=p \in \mathbb{C}[[X]]$, the right hand side being in $\mathbb{C}[X]_{\leq d-1}$, we get $f_{n+d}+$ $\sum_{i=1}^{d} q_{i} f_{n+d-i}=0$, for $n \in \mathbb{N}_{0}$, showing that $V_{(i)} \leq V_{(i i i)}$, hence $V_{(i)}=V_{(i i i)}$.

Choosing the coefficients of the $h_{j}$, for all $j \in\{1, \ldots, k\}$, yields $\operatorname{dim}_{\mathbb{C}}\left(V_{(i v)}\right) \leq$ $\sum_{j=1}^{k} d_{j}=d$. Letting $f \in V_{(i i)}$, we may assume that $f=\frac{X^{l}}{(1-a X)^{d}} \in \mathbb{C}[[X]]$, where $l \in\{0, \ldots, d-1\}$ and $0 \neq a \in \mathbb{C}$, thus $f=\sum_{n \geq 0}\left(\begin{array}{c}n+d-1 \\ d-1\end{array}\right)(a X)^{n} X^{l}=$ $a^{-l} \cdot \sum_{n \geq 0}\left(\begin{array}{c}n-l+d-1 \\ d-1\end{array}\right) a^{n} X^{n} \in \mathbb{C}[[X]]$, where the polynomial $\left(\begin{array}{c}X-l+d-1 \\ d-1\end{array}\right) \in \mathbb{C}[X]$ has degree $d-1$, showing that $V_{(i i)} \leq V_{(i v)}$, hence $V_{(i i)}=V_{(i v)}$.

Note that, if $f$ is given by a linear recursion of degree $d$, then the coefficients of $q \in \mathbb{C}[X]$ can be read off directly, while the coefficients of $p \in \mathbb{C}[X]_{\leq d-1}$ can be determined from $\left[f_{0}, \ldots, f_{d-1}\right]$ using $\left(\sum_{n \geq 0} f_{n} X^{n}\right)\left(1+\sum_{i=1}^{d} q_{i} X^{i}\right)=p=$ $\sum_{j=0}^{d-1} p_{j} X^{j} \in \mathbb{C}[[X]]$ as $p_{j}=f_{j}+\sum_{i=1}^{j} q_{i} f_{j-i} \in \mathbb{C}$, for $j \in\{0, \ldots, d-1\}$.

b) We have the equivalence of the following assertions, characterizing the degree of recurrence:

i) We have $\operatorname{gcd}(p, q)=1 \in \mathbb{C}[X]$.

ii) We have $\operatorname{deg}\left(h_{j}\right)=d_{j}-1$ for all $j \in\{1, \ldots, k\}$.

iii) The number $d \in \mathbb{N}$ is the smallest degree of recurrence of $f$.

c) Finally, we have the following characterization of polynomial maps, see (4.1): Given a map $h: \mathbb{N}_{0} \rightarrow \mathbb{C}$, then the following assertions are equivalent:

i) The map $h$ is polynomial of degree $d-1$.

ii) For the generating series we have $\sum_{n \geq 0} h(n) X^{n}=\frac{p}{(1-X)^{d}} \in \mathbb{C}(X) \cap \mathbb{C}[[X]]$, where $p \in \mathbb{C}[X]_{\leq d-1}$ such that $1-X \times p \in \mathbb{C}[X]$, that is $p(1) \neq 0 \in \mathbb{C}$.

In this case $p=\sum_{j=0}^{d-1} p_{j} X^{j} \in \mathbb{C}[X]_{\leq d-1}$ is called the Euler polynomial of $h$, its coefficients $\left[p_{0}, \ldots, p_{d-1}\right]$ are called the associated Euler numbers.

\section{Generating functions}

(13.1) Example: Fibonacci numbers. We consider the number $u_{n} \in \mathbb{N}_{0}$ of tilings of a $(2 \times n)$-rectangle by $(1 \times 2)$-rectangles, for $n \in \mathbb{N}_{0}$. Hence we have $u_{0}:=1$ and $u_{1}:=1$, and the linear recursion $u_{n+2}=u_{n+1}+u_{n}$ for $n \in \mathbb{N}_{0}$. Hence we have $u_{n}=F_{n+1}$ for $n \in \mathbb{N}_{0}$, where the $F_{n} \in \mathbb{N}_{0}$ are the Fibonacci numbers, see (0.1). 
To determine a closed formula for $F_{n}$ and $u_{n}$, we determine the generating series $F:=\sum_{n \geq 0} F_{n} X^{n} \in \mathbb{Q}[[X]]$ and $u:=\sum_{n \geq 0} u_{n} X^{n} \in \mathbb{Q}[[X]]:$ In view of the recursion we consider the polynomial $1-X-X^{2}=\left(1-\rho_{+} X\right)\left(1-\rho_{-} X\right) \in$ $\mathbb{R}[X]$, where $\rho_{ \pm}:=\frac{1}{2}(1 \pm \sqrt{5}) \in \mathbb{R}$. We have $F=\frac{a+b X}{1-X-X^{2}} \in \mathbb{Q}[[X]]$, where $a=F_{0}=0$ and $b=F_{1}-F_{0}=1$, hence $F=\frac{X}{1-X-X^{2}} \in \mathbb{Q}[[X]]$; thus we have $u=\sum_{n \geq 0} F_{n+1} X^{n}=\sum_{n \geq 1} F_{n} X^{n-1}=\frac{1}{1-X-X^{2}} \in \mathbb{Q}[[X]]$.

Partial fraction decomposition $\frac{1}{1-X-X^{2}}=\frac{\rho_{+}}{\sqrt{5}\left(1-\rho_{+} X\right)}-\frac{\rho_{-}}{\sqrt{5}\left(1-\rho_{-} X\right)} \in \mathbb{R}[[X]]$ yields $F=\frac{1}{\sqrt{5}} \cdot \sum_{n \geq 0}\left(\rho_{+}^{n+1}-\rho_{-}^{n+1}\right) X^{n+1}=\frac{1}{\sqrt{5}} \cdot \sum_{n \geq 0}\left(\rho_{+}^{n}-\rho_{-}^{n}\right) X^{n} \in \mathbb{R}[[X]]$, that is $F_{n}=\frac{1}{\sqrt{5}} \cdot\left(\rho_{+}^{n}-\rho_{-}^{n}\right) \in \mathbb{R}$, for all $n \in \mathbb{N}_{0}$; similarly, we get $u=\frac{1}{\sqrt{5}}$. $\sum_{n \geq 0}\left(\rho_{+}^{n+1}-\rho_{-}^{n+1}\right) X^{n}$, thus $u_{n}=\frac{1}{\sqrt{5}} \cdot\left(\rho_{+}^{n+1}-\rho_{-}^{n+1}\right)$, coinciding with $F_{n+1}$.

(13.2) Example: Catalan numbers. For $n \in \mathbb{N}_{0}$ let $c_{n} \in \mathbb{N}$ be the $n$-th Catalan number, which can be defined in numerous ways, for example as the number of non-associative words which can be formed from a sequence of $n+1$ letters, also called Schröder's (first) problem. We show that $c_{n}=\frac{1}{n+1} \cdot\left(\begin{array}{c}2 n \\ n\end{array}\right)$ :

Let $b_{n}:=c_{n-1} \in \mathbb{N}$ be the number of such words containing $n \in \mathbb{N}$ letters. Indicating the sequence of products taken by placing brackets, a word containing $n \geq 2$ letters is of the form $(\cdots)(\cdots)$, with factors containing $k$ and $n-k$ letters, respectively, where $k \in\{1, \ldots, n-1\}$. This yields the recursion $b_{n}=$ $\sum_{k=1}^{n-1} b_{k} b_{n-k}$, for $n \geq 2$, where $b_{1}=1$. For example, we have $b_{1}=b_{2}=1$ and $b_{3}=2$ and $b_{4}=5$ : For $n=1$ we have $\{\cdot\}$, for $n=2$ we have $\{\cdot \cdot\}$, for $n=3$ we have $\{\cdot(\cdot \cdot),(\cdot \cdot) \cdot\}$, and for $n=4$ we have $\{\cdot(\cdot(\cdot \cdot)), \cdot((\cdot \cdot) \cdot),(\cdot \cdot)(\cdot \cdot),(\cdot(\cdot \cdot)) \cdot,((\cdot) \cdot) \cdot\}$.

Let $b:=\sum_{n>0} b_{n} X^{n} \in \mathbb{Q}[[X]]$ be the associated generating series, where we let $b_{0}:=0$. Then the above recursion yields $b^{2}=\sum_{n \geq 0}\left(\sum_{k=0}^{n} b_{k} b_{n-k}\right) X^{n}=$ $\sum_{n \geq 2}\left(\sum_{k=1}^{n-1} b_{k} b_{n-k}\right) X^{n}=\sum_{n \geq 2} b_{n} X^{n}=b-X \in \mathbb{Q}[[X]]$, that is $b^{2}-b+X=$ $0 \in \mathbb{Q}[[X]]$. We look for a holomorphic map $\widehat{b}$, on a suitable open disc of positive radius centered at $x=0$, fulfilling the functional equation $\widehat{b}(x)^{2}-\widehat{b}(x)+x=0$. Solving the quadratic equation yields $\widehat{b}(x)=\frac{1}{2}(1-\sqrt{1-4 x})$, where the sign is chosen so that $\widehat{b}(0)=0$. Hence $\widehat{b}$ indeed is holomorphic for all $x \in \mathbb{C}$ such that $|x|<\frac{1}{4}$, and has the Taylor series expansion $\widehat{b}(x)=\sum_{n \geq 0} b_{n} x^{n}$ around $x=0$.

We have $(1-4 x)^{\frac{1}{2}}=\sum_{n \geq 0}\left(\begin{array}{c}\frac{1}{2} \\ n\end{array}\right)(-4 x)^{n}$ for all $x \in \mathbb{C}$ such that $|x|<\frac{1}{4}$. For $n \in \mathbb{N}_{0}$ we have $\left(\begin{array}{c}-\frac{1}{2} \\ n\end{array}\right)=\frac{(-1)^{n} \cdot \prod_{i=1}^{n}(2 i-1)}{2^{n} \cdot n !}=\frac{(-1)^{n} \cdot(2 n) !}{2^{2 n} \cdot(n !)^{2}}=\left(\frac{-1}{4}\right)^{n} \cdot\left(\begin{array}{c}2 n \\ n\end{array}\right)$; see also Exercise (19.12). This for $n \geq 1$ yields $\left(\begin{array}{c}\frac{1}{2} \\ n\end{array}\right)=\frac{1}{2 n} \cdot\left(\begin{array}{c}-\frac{1}{2} \\ n-1\end{array}\right)=\frac{(-1)^{n-1}}{2^{2 n-1} n} \cdot\left(\begin{array}{c}2 n-2 \\ n-1\end{array}\right)$. Thus we get $\widehat{b}(x)=-\frac{1}{2} \cdot \sum_{n \geq 1} \frac{(-1)^{n-1}(-4)^{n}}{2^{2 n-1} n} \cdot\left(\begin{array}{c}2 n-2 \\ n-1\end{array}\right) x^{n}=\sum_{n \geq 1} \frac{(2 n-2) !}{n !(n-1) !} x^{n}$; note that it is not at all obvious how to show directly the convergence of the right hand side on an open disc of positive radius centered at $x=0$. Hence we have $b=\sum_{n \geq 1} \frac{(2 n-2) !}{n !(n-1) !} X^{n}=\sum_{n \geq 1} \frac{1}{n} \cdot\left(\begin{array}{c}2 n-2 \\ n-1\end{array}\right) X^{n} \in \mathbb{Q}[[X]]$. 
(13.3) Exponential generating series. a) Let $K$ be a field of characteristic 0 . For a sequence $\left[f_{n} \in K ; n \in \mathbb{N}_{0}\right]$ let $\widetilde{f}:=\sum_{n \geq 0} \frac{f_{n}}{n !} X^{n} \in K[[X]]$ be the associated exponential generating series. In particular, differentiation yields $\frac{\partial}{\partial X} \widetilde{f}=\sum_{n \geq 1} \frac{n f_{n}}{n !} X^{n-1}=\sum_{n \geq 0} \frac{f_{n+1}}{n !} X^{n} \in K[[X]]$, which amounts to forming the exponential generating series of a shift of the given sequence.

Given $\tilde{f}=\sum_{n \geq 0} \frac{f_{n}}{n !} X^{n} \in K[[X]]$ and $\widetilde{g}=\sum_{n \geq 0} \frac{g_{n}}{n !} X^{n} \in K[[X]]$, and letting $\widetilde{h}:=\widetilde{f} \widetilde{g}=\sum_{n \geq 0} \frac{h_{n}}{n !} X^{n} \in K[[X]]$, convolutional multiplication becomes $\widetilde{h}=$ $\sum_{n \geq 0}\left(\sum_{k=0}^{n} \frac{n !}{k !(n-k) !} f_{k} g_{n-k}\right) \cdot \frac{1}{n !} X^{n}$, saying that $h_{n}=\sum_{k=0}^{n}\left(\begin{array}{l}n \\ k\end{array}\right) f_{k} g_{n-k} \in K$, for all $n \in \mathbb{N}_{0}$, that is the sequence $\left[h_{n} \in K ; n \in \mathbb{N}_{0}\right]$ is given as binomial convolution of the sequences $\left[f_{n} \in K ; n \in \mathbb{N}_{0}\right]$ and $\left[g_{n} \in K ; n \in \mathbb{N}_{0}\right]$.

For example, considering the sequence $\left[a_{(n)} ; n \in \mathbb{N}_{0}\right]$ of falling factorials associated with $a \in K$ we get $\sum_{n \geq 0} \frac{a_{(n)}}{n !} X^{n}=\sum_{n \geq 0}\left(\begin{array}{l}a \\ n\end{array}\right) X^{n}=(1+X)^{a} \in K[[X]]$; hence from the identity $(1+X)^{a+b}=(1+X)^{a}(1+X)^{b} \in K[[X]]$, for $a, b \in K$, we recover the formula $(a+b)_{(n)}=\sum_{k=0}^{n}\left(\begin{array}{l}n \\ k\end{array}\right) a_{(k)} b_{(n-k)} \in K$, that is the Vandermonde identity $\left(\begin{array}{c}a+b \\ n\end{array}\right)=\sum_{k=0}^{n}\left(\begin{array}{l}a \\ k\end{array}\right)\left(\begin{array}{c}b \\ n-k\end{array}\right) \in K$, for all $n \in \mathbb{N}_{0}$.

b) The exponential generating series of the geometric series associated with $a \in K$ is given as $\sum_{n>0} \frac{a^{n}}{n !} X^{n}=\exp (a X) \in K[[X]]$; hence from the identity $\exp ((a+b) X)=\exp (a X) \exp (b X) \in K[[X]]$, for $a, b \in K$, we recover the binomial formula $(a+b)^{n}=\sum_{k=0}^{n}\left(\begin{array}{l}n \\ k\end{array}\right) a^{k} b^{n-k} \in K$, for all $n \in \mathbb{N}_{0}$.

In particular, using the constant series associated with $a:=1$ we get the following: For sequences $\left[f_{n} \in K ; n \in \mathbb{N}_{0}\right]$ and $\left[g_{n} \in K ; n \in \mathbb{N}_{0}\right]$ being related by $g_{n}=\sum_{k=0}^{n}\left(\begin{array}{l}n \\ k\end{array}\right) f_{k} \in K$, for all $n \in \mathbb{N}_{0}$, we have $\widetilde{g}=\widetilde{f} \cdot \exp \in K[[X]]$, implying $\widetilde{f}=\widetilde{g} \cdot \exp (X)^{-1}=\widetilde{g} \cdot \exp (-X) \in K[[X]]$, from which we in turn recover the binomial inversion formula $f_{n}=\sum_{k=0}^{n}(-1)^{n-k}\left(\begin{array}{l}n \\ k\end{array}\right) g_{k} \in K$, for all $n \in \mathbb{N}_{0}$.

For example, to determine the exponential generating series $\widetilde{D} \in \mathbb{Q}[[X]]$ of the sequence $\left[D_{n} ; n \in \mathbb{N}_{0}\right]$ of derangement numbers we proceed as follows: We have $\sum_{k=0}^{n}\left(\begin{array}{l}n \\ k\end{array}\right) D_{n-k}=n$ !, for all $n \in \mathbb{N}_{0}$, hence $\widetilde{D} \cdot \exp =\sum_{n \geq 0} \frac{n !}{n !} X^{n}=(1-X)^{-1} \in$ $\mathbb{Q}[[X]]$, thus $\widetilde{D}=\frac{\exp (-X)}{1-X} \in \mathbb{Q}[[X]]$; in particular, from this we recover the formula $D_{n}=\sum_{k=0}^{n} \frac{(-1)^{k}}{k !} \in \mathbb{Q}$, for all $n \in \mathbb{N}_{0}$.

(13.4) Example: Stirling numbers of the second kind. a) For $n, k \in \mathbb{N}_{0}$ let $S_{n, k} \in \mathbb{N}_{0}$ be the associated Stirling number of the second kind, and let $S_{k}:=\sum_{n \geq 0} S_{n, k} X^{n} \in \mathbb{Q}[[X]]$ be the associated generating series, where hence $S_{0}=1$. We show that $S_{k}=\prod_{l=1}^{k} \frac{X}{1-l X} \in \mathbb{Q}[[X]]$, for all $k \in \mathbb{N}_{0}$ :

We proceed by induction on $k \in \mathbb{N}_{0}$; the assertion being true for $k=0$, let $k \geq 1$. The recursion $S_{n, k}=S_{n-1, k-1}+k \cdot S_{n-1, k}$, for $n, k \geq 1$, yields $S_{k}=$ $X \cdot\left(\sum_{n \geq k} S_{n-1, k-1} X^{n-1}+k \cdot \sum_{n \geq k+1} S_{n-1, k} X^{n-1}\right)=X\left(S_{k-1}+k S_{k}\right) \in \mathbb{Q}[[X]]$, thus $(1-k X) S_{k}=X S_{k-1} \in \mathbb{Q}[[X]]$, implying $S_{k}=\frac{X}{1-k X} \cdot S_{k-1}=\frac{X}{1-k X}$. $\prod_{l=1}^{k-1} \frac{X}{1-l X}=\prod_{l=1}^{k} \frac{X}{1-l X} \in \mathbb{Q}[[X]]$. 
b) Let $\widetilde{S}_{k}:=\sum_{n \geq 0} \frac{S_{n, k}}{n !} X^{n} \in \mathbb{Q}[[X]]$ be the associated exponential generating series, where $\widetilde{S}_{0}=1$. We show that $\widetilde{S}_{k}=\frac{1}{k !}(\exp -1)^{k} \in \mathbb{Q}[[X]]$, for all $k \in \mathbb{N}_{0}$ : The assertion being true for $k=0$, let $k \geq 1$. The recursion $S_{n, k}=S_{n-1, k-1}+$ $k \cdot S_{n-1, k}$, for $n, k \geq 1$, yields $\widetilde{S}_{k}=\sum_{n \geq k} \frac{S_{n-1, k-1}}{n !} X^{n}+k \cdot \sum_{n \geq k+1} \frac{S_{n-1, k}}{n !} X^{n} \in$ $\mathbb{Q}[[X]]$, leading to the differential equation $\frac{\partial}{\partial X} \widetilde{S}_{k}=\sum_{n \geq k} \frac{S_{n-1, k-1}^{-1}}{(n-1) !} X^{n-1}+k$. $\sum_{n \geq k+1} \frac{S_{n-1, k}}{(n-1) !} X^{n-1}=\widetilde{S}_{k-1}+k \widetilde{S}_{k} \in \mathbb{Q}[[X]]$. Since $\widetilde{S}_{k-1} \in \mathbb{Q}[[X]]$ is known, and the constant term of $\widetilde{S}_{k}$ is $S_{0, k}=0$, by successively comparing coefficients we conclude that $\widetilde{S}_{k} \in \mathbb{Q}[[X]]$ is uniquely determined; note that this is also follows from the fact that the differential equation $\frac{\partial}{\partial X} \widehat{\widetilde{S}}_{k}(x)=\widehat{\widetilde{S}}_{k-1}(x)+k \widehat{\widetilde{S}}_{k}(x)$, given any initial value $\widehat{\widetilde{S}}_{k}(0) \in \mathbb{C}$, has a unique solution in an open disc of positive radius centered at $x=0$. Hence it suffices to show that $\widetilde{T}_{k}:=\frac{1}{k !}(\exp -1)^{k} \in \mathbb{Q}[[X]]$ is a solution of the above equation: We have $\frac{\partial}{\partial X} \widetilde{T}_{k}=\frac{1}{(k-1) !}(\exp -1)^{k-1} \exp =$ $\frac{1}{(k-1) !}(\exp -1)^{k-1}+\frac{k}{k !}(\exp -1)^{k}=\widetilde{T}_{k-1}+k \widetilde{T}_{k} \in \mathbb{Q}[[X]]$.

Note that $\widetilde{S}_{k}=\sum_{n>0} \frac{S_{n, k}}{n !} X^{n}=\frac{1}{k !}(\exp -1)^{k}=\frac{1}{k !} \cdot \sum_{l=0}^{k}(-1)^{k-l}\left(\begin{array}{l}k \\ l\end{array}\right) \exp (l X)=$ $\frac{1}{k !} \cdot \sum_{l=0}^{k}\left((-1)^{k-l}\left(\begin{array}{l}k \\ l\end{array}\right) \cdot \sum_{n \geq 0} \frac{l^{n}}{n !} X^{n}\right)=\frac{1}{k !} \cdot \sum_{n \geq 0}\left(\sum_{l=0}^{k}(-1)^{k-l}\left(\begin{array}{l}k \\ l\end{array}\right) l^{n}\right) \frac{X^{n}}{n !} \in \mathbb{Q}[[X]]$ yields the sum formula $S_{n, k}=\frac{1}{k !} \cdot \sum_{l=0}^{k}(-1)^{k-l}\left(\begin{array}{l}k \\ l\end{array}\right) l^{n} \in \mathbb{Q}$ again.

c) Let $B_{n}:=\sum_{k=0}^{n} S_{n, k} \in \mathbb{N}$ be the $n$-th Bell number. The associated exponential generating series is $\widetilde{B}:=\sum_{n \geq 0} \frac{B_{n}}{n !} X^{n}=\sum_{k \geq 0} \widetilde{S}_{k}=\sum_{k \geq 0} \frac{1}{k !}(\exp -1)^{k}=$ $\exp (\exp -1) \in \mathbb{Q}[[X]]$. Thus we get $\sum_{n \geq 0} \frac{B_{n+1}}{n !} X^{n}=\frac{\partial}{\partial X} \widetilde{B}=\exp (\exp -1)$. $\exp =\widetilde{B} \cdot \exp =\sum_{n \geq 0}\left(\sum_{k=0}^{n} \frac{B_{k}}{k !(n-k) !}\right) X^{n} \in \mathbb{Q}[[X]]$, and comparing coefficients yields $B_{n+1}=\sum_{k=0}^{n}\left(\begin{array}{l}n \\ k\end{array}\right) B_{k}$, for all $n \in \mathbb{N}_{0}$; for a combinatorial proof see (19.23).

The Taylor series $\widehat{\widetilde{B}}(x)$ converges for all $x \in \mathbb{C}$. This yields $\sum_{n \geq 0} B_{n} \cdot \frac{x^{n}}{n !}=$ $\widehat{\widetilde{B}}(x)=\exp (\exp (x)-1)=\frac{1}{e} \cdot \exp (\exp (x))=\frac{1}{e} \cdot \sum_{k \geq 0} \frac{\exp (x)^{k}}{k !}=\frac{1}{e} \cdot \sum_{k \geq 0} \frac{\exp (k x)}{k !}=$ $\frac{1}{e} \cdot \sum_{k \geq 0}\left(\frac{1}{k !} \cdot \sum_{n \geq 0} \frac{(k x)^{n}}{n !}\right)=\frac{1}{e} \cdot \sum_{n \geq 0}\left(\sum_{k \geq 0} \frac{k^{n}}{k !}\right) \cdot \frac{x^{n}}{n !} \in \mathbb{C}$, for all $x \in \mathbb{C}$, hence $B_{n}=\frac{1}{e} \cdot \sum_{k \geq 0} \frac{k^{n}}{k !} \in \mathbb{C}$; note that this cannot be translated to the formal setting.

(13.5) Example: Stirling numbers of the first kind. For $n, k \in \mathbb{N}_{0}$ let $s_{n, k} \in \mathbb{N}_{0}$ be the associated Stirling number of the first kind, and let $\widetilde{s}_{k}:=$ $\sum_{n \geq 0} \frac{s_{n, k}}{n !} X^{n} \in \mathbb{Q}[[X]]$ be the associated exponential generating series, where hence $\widetilde{s}_{0}=1$. We show that $\widetilde{s}_{k}=\frac{(-1)^{k}}{k !} \log (-X)^{k} \in \mathbb{Q}[[X]]$, for all $k \in \mathbb{N}_{0}$ :

We proceed by induction on $k \in \mathbb{N}_{0}$; the assertion being true for $k=0$, let $k \geq 1$. The recursion $s_{n, k}=s_{n-1, k-1}+(n-1) \cdot s_{n-1, k}$ yields $\widetilde{s}_{k}=\sum_{n \geq k} \frac{s_{n-1, k-1}}{n !} X^{n}+$ $\sum_{n \geq k+1} \frac{(n-1) s_{n-1, k}}{n !} X^{n} \in \mathbb{Q}[[X]]$, thus we get $\frac{\partial}{\partial X} \widetilde{s}_{k}=\sum_{n \geq k} \frac{s_{n-1, k-1}}{(n-1) !} X^{n-1}+$ $\sum_{n \geq k+1} \frac{s_{n-1, k}}{(n-2) !} X^{n-1} \in \mathbb{Q}[[X]]$. Recall that the above recursion entails the identity $\frac{s_{n, k}}{(n-1) !}=\sum_{m=0}^{n-1} \frac{s_{m, k-1}}{m !}$, thus we get $\widetilde{s}_{k-1} \cdot\left(\frac{1}{1-X}-1\right)=\left(\sum_{n \geq 0} \frac{s_{n, k-1}}{n !} X^{n}\right)$. $\left(\sum_{l \geq 1} X^{l}\right)=\sum_{n \geq 1}\left(\sum_{m=0}^{n-1} \frac{s_{m, k-1}}{m !}\right) X^{n}=\sum_{n \geq 1} \frac{s_{n, k}}{(n-1) !} X^{n} \in \mathbb{Q}[[X]]$, hence we ar- 
rive at the differential equation $\frac{\partial}{\partial X} \widetilde{s}_{k}=\widetilde{s}_{k-1}+\widetilde{s}_{k-1} \cdot\left(\frac{1}{1-X}-1\right)=\frac{1}{1-X} \cdot \widetilde{s}_{k-1} \in$ $\mathbb{Q}[[X]]$, which determines $\widetilde{s}_{k}$ uniquely, since its constant term is known to be $s_{0, k}=0$. Thus it suffices to show that $\widetilde{t}_{k}:=\frac{(-1)^{k}}{k !} \log (-X)^{k} \in \mathbb{Q}[[X]]$ is a solution: We have $\frac{\partial}{\partial X} \widetilde{t}_{k}=\frac{(-1)^{k}}{(k-1) !} \log (-X)^{k-1} \cdot \frac{-1}{1-X}=\frac{1}{1-X} \cdot \widetilde{t}_{k-1} \in \mathbb{Q}[[X]] . \quad \sharp$

\section{Partition identities}

(14.1) Partitions. a) Let $p_{n, k}:=\left|P_{k}(n)\right| \in \mathbb{N}_{0}$ be the number of partitions of $n \in \mathbb{N}_{0}$ into $k \in \mathbb{N}_{0}$ parts, let $p_{n, \leq k}:=\left|P_{\leq k}(n)\right|=\sum_{l=0}^{k} p_{n, l} \in \mathbb{N}_{0}$ be the number of partitions of $n$ into at most $k$ parts, and let $p_{n}:=|P(n)|=$ $\sum_{k=0}^{n} p_{n, k} \in \mathbb{N}_{0}$ be the number of all partitions of $n$; recall that $p_{n, k}=0$ for $k>n$. These numbers are encoded into the weighted generating series $P:=\sum_{n>0}\left(\sum_{k=0}^{n} p_{n, k} Y^{k}\right) X^{n} \in \mathbb{Q}[Y][[X]]$, hence the ordinary generating series $p:=\sum_{n \geq 0} p_{n} X^{n} \in \mathbb{Q}[[X]]$ is obtained by evaluating $P$ at $y:=1$. We let $P_{k}:=$ $\sum_{n \geq k} p_{n, k} X^{n} \in X^{k} \mathbb{Q}[[X]]$ and $P_{\leq k}:=\sum_{n \geq 0} p_{n, \leq k} X^{n}=\sum_{l=0}^{k} P_{k} \in \mathbb{Q}[[X]]$, hence $P=\sum_{k \geq 0}\left(\sum_{n \geq k} p_{n, k} X^{n}\right) Y^{k}=\sum_{k \geq 0} P_{k} Y^{k} \in \mathbb{Q}[[X]][[Y]]$.

Writing partitions in terms of multiplicities as $\lambda=\left[n^{a_{n}(\lambda)}, \ldots, 1^{a_{1}(\lambda)}\right] \vdash n$, the map $\lambda \mapsto\left[a_{1}(\lambda), a_{2}(\lambda), \ldots\right]$ is a bijection from the set $\coprod_{n \geq 0} P(n)$ of all partitions to the set $\mathcal{M}:=\left\{\left[a_{1}, a_{2}, \ldots\right] \in \operatorname{Maps}\left(\mathbb{N}, \mathbb{N}_{0}\right) ; a_{i}=0\right.$ for almost all $i \in$ $\mathbb{N}\}$; hence $a \in \mathcal{M}$ corresponds to a partition $\lambda(a)$ of $|\lambda(a)|=\sum_{i>1} i a_{i}$ with $l(\lambda(a))=\sum_{i \geq 1} a_{i}$ parts. Using this we get $P=\sum_{n \geq 0}\left(\sum_{\lambda \vdash n} Y^{l(\lambda)}\right) X^{n}=$ $\sum_{a \in \mathcal{M}} Y^{l(\lambda(a))} X^{|\lambda(a)|}=\sum_{a \in \mathcal{M}} Y^{\sum_{i \geq 1} a_{i}} X^{\sum_{i \geq 1} i a_{i}}=\sum_{a \in \mathcal{M}} \prod_{i \geq 1}\left(Y X^{i}\right)^{a_{i}}=$ $\prod_{i \geq 1}\left(\sum_{j \geq 0}\left(Y X^{i}\right)^{j}\right)=\prod_{i \geq 1} \frac{1}{1-Y X^{i}} \in \mathbb{Q}[Y][[X]]$. Hence evaluating at $y:=1$ yields $p=\prod_{i \geq 1} \frac{1}{1-X^{i}} \in \mathbb{Q}[[X]]$.

It turns out that $p \in \mathbb{Q}[[X]]$ has a close relation to number theory: We have $\log (p-1)=-\sum_{n \geq 1} \log \left(-X^{n}\right) \in \mathbb{Q}[[X]]$, hence we get $X \cdot \frac{\partial}{\partial X}(\log (p-1))=$ $\sum_{n \geq 1} \frac{n X^{n}}{1-X^{n}}=\sum_{n \geq 1}\left(n \cdot \sum_{k \geq 1} X^{k n}\right)=\sum_{n \geq 1}\left(\sum_{d \mid n} d\right) X^{n}=\sum_{n \geq 1} \sigma(n) X^{n}=$ : $\sigma \in \mathbb{Q}[[X]]$, where $\sigma(n):=\sum_{d \mid n} d \in \mathbb{N}$ is the sum of the divisors of $n \in \mathbb{N}$. Since $X \cdot \frac{\partial}{\partial X}(\log (p-1))=\frac{X}{p} \cdot \frac{\partial}{\partial X}(p) \in \mathbb{Q}[[X]]$ is a shifted logarithmic derivative of $p$, we obtain $X \cdot \frac{\partial}{\partial X}(p)=p \cdot \sigma \in \mathbb{Q}[[X]]$, yielding $\sum_{n \geq 1} n p_{n} X^{n}=\left(\sum_{n \geq 0} p_{n} X^{n}\right)$. $\left(\sum_{n \geq 1} \sigma(n) X^{n}\right)=\sum_{n \geq 1}\left(\sum_{i=1}^{n} \sigma(i) p_{n-i}\right) X^{n} \in \mathbb{Q}[[X]]$. Thus we have proved the recursion $n \cdot p_{n}=\sum_{i=1}^{n} \sigma(i) \cdot p_{n-i}$ for all $n \in \mathbb{N}_{0}$.

b) We determine $P_{\leq k} \in \mathbb{Q}[[X]]$ and $P_{k} \in \mathbb{Q}[[X]]$. To this end, we write $\lambda \in$ $P_{\leq k}(n)$ as $\lambda=\left[\lambda_{1}, \ldots, \lambda_{k}\right]$ where $\lambda_{1} \geq \cdots \geq \lambda_{k} \geq 0$, and let $P_{\leq k}^{\geq j}(n):=$ $\left\{\lambda \in P_{\leq k}(n) ; \lambda_{k} \geq j\right\}$, for $j \in \mathbb{N}_{0}$. For the associated generating series we

show by induction on $k \in \mathbb{N}_{0}$ that $P_{\leq k}^{\geq j}=\prod_{i=1}^{k} \frac{X^{j}}{1-X^{i}} \in \mathbb{Q}[[X]]$ : The case $k=0$ being trivial, let $k \geq 1$. Then we have $P_{\leq k}^{\geq j}=\sum_{\lambda_{1} \geq \cdots \geq \lambda_{k} \geq j} X^{\sum_{i=1}^{k} \lambda_{i}}=$ $\sum_{\lambda_{k} \geq j}\left(X^{\lambda_{k}} \cdot \sum_{\lambda_{1} \geq \cdots \geq \lambda_{k-1} \geq \lambda_{k}} X^{\sum_{i=1}^{k-1} \lambda_{i}}\right)$, thus by induction $P_{\leq k}^{\geq j}=\sum_{\lambda_{k} \geq j}\left(X^{\lambda_{k}}\right.$. $\left.\prod_{i=1}^{k-1} \frac{X^{\lambda_{k}}}{1-X^{i}}\right)=\left(\prod_{i=1}^{k-1} \frac{1}{1-X^{i}}\right) \cdot \sum_{\lambda_{k} \geq j} X^{k \lambda_{k}}=\left(\prod_{i=1}^{k-1} \frac{1}{1-X^{i}}\right) \cdot \frac{X^{j k}}{1-X^{k}}=\prod_{i=1}^{k} \frac{X^{j}}{1-X^{i}}$. 
Thus in particular we get $P_{\leq k}=P_{\leq k}^{\geq 0}=\prod_{i=1}^{k} \frac{1}{1-X^{i}} \in \mathbb{Q}[[X]]$ and $P_{k}=$ $P_{\leq k}^{\geq 1}=\prod_{i=1}^{k} \frac{X}{1-X^{i}} \in \mathbb{Q}[[X]]$. Note that this implies $p=\lim _{k \rightarrow \infty} P_{\leq k}=$ $\prod_{i \geq 1} \frac{1}{1-X^{i}} \in \mathbb{Q}[[X]]$. Moreover, for $k \geq 1$ we get $P_{k}=P_{\leq k}-P_{\leq k-1}=$ $\left(\frac{1}{1-X^{k}}-1\right) \cdot \prod_{i=1}^{k-1} \frac{1}{1-X^{i}}=X^{k} \cdot \prod_{i=1}^{k} \frac{1}{1-X^{i}}=X^{k} \cdot P_{\leq k} \in \mathbb{Q}[[X]]$, while $P_{0}=$ $P_{\leq 0}=1 \in \mathbb{Q}[[X]]$ anyway, which is reminiscent of the bijection $P_{k}(n) \rightarrow$ $P_{\leq k}(n-k):\left[\lambda_{1}, \ldots, \lambda_{k}\right] \mapsto\left[\lambda_{1}-1, \ldots, \lambda_{k}-1\right]$. Finally, this implies the partition identity $\prod_{i \geq 1} \frac{1}{1-Y X^{i}}=P=\sum_{k \geq 0} P_{k} Y^{k}=\sum_{k \geq 0}\left(\prod_{i=1}^{k} \frac{X}{1-X^{i}}\right) Y^{k}=$ $\sum_{k \geq 0} \prod_{i=1}^{k} \frac{X Y}{1-X^{i}}=\sum_{k \geq 0}\left(\prod_{i=1}^{k} \frac{1}{1-X^{i}}\right) X^{k} Y^{k} \in \mathbb{Q}[[X]][[Y]]$.

(14.2) Regular partitions. a) Let $n \in \mathbb{N}_{0}$ and $k \in \mathbb{N}_{0}$. For $r \geq 2$ let $P_{r^{\prime}, k}(n) \subseteq P_{k}(n)$ be the set of partitions of $n$ consisting of $k$ parts none of which is divisible by $r$, and let $P_{r \text {-reg, } k}(n) \subseteq P_{k}(n)$ be the set of $r$-regular $k$-part partitions of $n$, that is those all of whose parts have multiplicity less than $r$. Then we have $P_{r^{\prime}}(n)=\coprod_{k=0}^{n} P_{r^{\prime}, k}(n)$ and $P_{r \text {-reg }}(n)=\coprod_{k=0}^{n} P_{r \text {-reg, }, k}(n)$. For $r=2$ we get the sets $O_{k}(n):=P_{2^{\prime}, k}(n)$ of partitions of $n$ consisting of $k$ odd parts, and $D_{k}(n):=P_{2 \text {-reg, } k}(n)$ of partitions of $n$ having $k$ pairwise distinct parts; hence $O(n)=\coprod_{k=0}^{n} O_{k}(n)$ and $D(n)=\coprod_{k=0}^{n} D_{k}(n)$.

Then $\coprod_{n>0} P_{r^{\prime}}(n)$ corresponds to $\mathcal{M}_{r^{\prime}}:=\left\{a \in \mathcal{M} ; a_{r i}=0\right.$ for all $\left.i \in \mathbb{N}\right\}$, which yields $P_{r^{\prime}}:=\sum_{n \geq 0}\left(\sum_{k=0}^{n}\left|P_{r^{\prime}, k}(n)\right| Y^{k}\right) X^{n}=\sum_{a \in \mathcal{M}_{r^{\prime}}} \prod_{i \geq 1}\left(Y X^{i}\right)^{a_{i}}=$ $\prod_{i \geq 1, r \chi i}\left(\sum_{j \geq 0}\left(Y X^{i}\right)^{j}\right)=\prod_{i \geq 1, r \chi i} \frac{1}{1-Y X^{i}}=\prod_{i \geq 1} \frac{1-Y X^{r i}}{1-Y X^{i}} \in \mathbb{Q}[Y][[X]]$ and thus $p_{r^{\prime}}:=\sum_{n \geq 0}\left|P_{r^{\prime}}(n)\right| X^{n}=\prod_{i \geq 1} \frac{1-X^{r i}}{1-X^{i}} \in \mathbb{Q}[[X]]$ as weighted and ordinary generating series, respectively; for $r=2$ we get $p_{2^{\prime}}=\prod_{i \geq 1}\left(1+X^{i}\right) \in \mathbb{Q}[[X]]$.

Then $\coprod_{n \geq 0} P_{r \text {-reg }}(n)$ corresponds to $\mathcal{M}_{r \text {-reg }}:=\left\{a \in \mathcal{M} ; a_{i}<r\right.$ for all $\left.i \in \mathbb{N}\right\}$, thus $P_{r \text {-reg }}:=\sum_{n \geq 0}\left(\sum_{k=0}^{n}\left|P_{r \text {-reg, } k}(n)\right| Y^{k}\right) X^{n}=\sum_{a \in \mathcal{M}_{r-\text { reg }}} \prod_{i \geq 1}\left(Y X^{i}\right)^{a_{i}}=$ $\prod_{i \geq 1}\left(\sum_{j=0}^{r-1}\left(Y X^{i}\right)^{j}\right)=\prod_{i \geq 1} \frac{1-\left(Y X^{i}\right)^{r}}{1-Y X^{i}} \in \mathbb{Q}[Y][[X]]$ and hence we get $p_{r-\text { reg }}:=$ $\sum_{n>0}\left|P_{r-\mathrm{reg}}(n)\right| X^{n}=\prod_{i>1} \frac{1-X^{i r}}{1-X^{i}} \in \mathbb{Q}[[X]]$ as weighted and ordinary generating series, respectively. Thus we recover $\left|P_{r^{\prime}}(n)\right|=\left|P_{r \text {-reg }}(n)\right|$ for all $n \in \mathbb{N}_{0}$.

b) In particular, for $r=2$ wet get $D:=P_{2 \text {-reg }}=\prod_{i \geq 1} \frac{1-\left(Y X^{i}\right)^{2}}{1-Y X^{i}}=\prod_{i \geq 1}(1+$ $\left.Y X^{i}\right) \in \mathbb{Q}[Y][[X]]$. The bijection $D_{k}(n) \rightarrow P_{\leq k}\left(n-\left(\begin{array}{c}k+1 \\ 2\end{array}\right)\right):\left[\lambda_{1}, \ldots, \lambda_{k}\right] \mapsto$ $\left[\lambda_{1}-k, \lambda_{1}-(k-1), \ldots, \lambda_{k}-1\right]$ yields $D_{k}:=\sum_{n \geq 0}\left|D_{k}(n)\right| X^{n}=X^{\left(\begin{array}{c}k+1 \\ 2\end{array}\right)} \cdot P_{\leq k}=$ $\prod_{i=1}^{k} \frac{X^{i}}{1-X^{i}} \in \mathbb{Q}[[X]]$, yielding the identity $\prod_{i \geq 1}\left(1+Y X^{i}\right)=D=\sum_{k \geq 0} D_{k} Y^{k}=$ $\sum_{k \geq 0}\left(\prod_{i=1}^{k} \frac{X^{i}}{1-X^{i}}\right) Y^{k}=\sum_{k \geq 0}\left(\prod_{i=1}^{k} \frac{1}{1-X^{i}}\right) X^{\left({ }^{k+1}\right)} Y^{k} \in \mathbb{Q}[[X]][[Y]]$.

Moreover, let $C_{k}(n):=D_{k}(n) \cap O_{k}(n)$ be the set of partitions of $n$ consisting of $k$ pairwise distinct odd parts, and let $C(n):=\coprod_{k>0} C_{k}(n)$. Then $\coprod_{n>0} C(n)$ corresponds to $\mathcal{M}_{C}:=\left\{a \in \mathcal{M} ; a_{2 i}=0, a_{2 i-1} \leq 1\right.$ for all $\left.i \in \mathbb{N}\right\}$. Hence we get $C:=\sum_{n>0}\left(\sum_{k=0}^{n}\left|C_{k}(n)\right| Y^{k}\right) X^{n}=\sum_{a \in \mathcal{M}_{C}} \prod_{i \geq 1}\left(Y X^{i}\right)^{a_{i}}=\prod_{i \geq 1}(1+$ $\left.Y X^{2 i-1}\right) \in \mathbb{Q}[Y][[X]]$, thus $c:=\sum_{n \geq 0}|C(n)| X^{n}=\prod_{i \geq 1}\left(1+X^{2 i-1}\right) \in \mathbb{Q}[[X]]$. Note that evaluating $D \in \mathbb{Q}[[X]][[Y]]$ at $X \mapsto X^{2}$ and $Y \mapsto \frac{Y}{X}$ yields $C=$ $\prod_{i \geq 1}\left(1+Y X^{2 i-1}\right)=\sum_{k \geq 0}\left(\prod_{i=1}^{k} \frac{1}{1-X^{2 i}}\right) X^{k^{2}} Y^{k} \in \mathbb{Q}((X))[[Y]]$. 
(14.3) Conjugate partitions. a) Let $n \in \mathbb{N}_{0}$ and $k \in \mathbb{N}_{0}$. Let $P^{k}(n):=$ $\left\{\lambda \in P(n) ; \lambda_{1}=k\right\}$ be the set of all partitions of $n$ whose largest part is $k$, and $P^{\leq k}(n):=\coprod_{l=0}^{k} P^{l}(n)$ be the those whose largest part is at most $k$, and let $p_{n}^{k}:=\left|P^{k}(n)\right| \in \mathbb{N}_{0}$ and $p_{n}^{\leq k}:=\left|P^{\leq k}(n)\right|=\sum_{l=0}^{k} p_{n}^{l} \in \mathbb{N}_{0}$.

Then $\coprod_{n \geq 0} P \leq k(n)$ corresponds to $\mathcal{M} \leq k:=\operatorname{Maps}\left(\{1, \ldots, k\}, \mathbb{N}_{0}\right)$, thus $P \leq k:=$ $\sum_{n \geq 0} p_{\bar{n}}^{\leq k} X^{n}=\sum_{a \in \mathcal{M} \leq k} \prod_{i=1}^{k} X^{i a_{i}}=\prod_{i=1}^{k} \frac{1}{1-X^{i}} \in \mathbb{Q}[[X]]$. And $\coprod_{n \geq 0} P^{k}(n)$ corresponds to $\mathcal{M}^{=k}:=\left\{a \in \mathcal{M}^{\leq k} ; a_{k} \geq 1\right\}$, thus $P^{=k}:=\sum_{n \geq 0} p_{n}^{k} X^{n}=$ $\sum_{a \in \mathcal{M}=k} \prod_{i=1}^{k} X^{i a_{i}}=X^{k} \cdot \prod_{i=1}^{k} \frac{1}{1-X^{i}}=\prod_{i=1}^{k} \frac{X}{1-X^{i}} \in \mathbb{Q}[[X]] ;$ alternatively, we get $P^{=k}=P^{\leq k}-P^{\leq(k-1)}=\left(\frac{1}{1-X^{k}}-1\right) \cdot \prod_{i=1}^{k-1} \frac{1}{1-X^{i}}=X^{k} \cdot \prod_{i=1}^{k} \frac{1}{1-X^{i}} \in \mathbb{Q}[[X]]$, for $k \geq 1$. Hence for all $k \in \mathbb{N}_{0}$ we have $P^{=k}=P_{k} \in \mathbb{Q}[[X]]$ and $P \leq k=P_{\leq k} \in$ $\mathbb{Q}[[X]]$, for all $k \in \mathbb{N}_{0}$, that is $p_{n}^{k}=p_{n, k}$ and $p_{n}^{\leq k}=p_{n, \leq k}$, for all $n \in \mathbb{N}_{0}$.

This can also be deduced combinatorially from taking conjugate partitions: If $\lambda \vdash n$ has $k$ parts then we get $\lambda_{1}^{\prime}=k$; and if $\lambda_{1}=k$ then we get $\lambda_{k}^{\prime}=\mid\{j \in$ $\left.\mathbb{N} ; \lambda_{j} \geq k\right\} \mid>0$ and $\lambda_{k+1}^{\prime}=\left|\left\{j \in \mathbb{N} ; \lambda_{j} \geq k+1\right\}\right|=0$, saying that $\lambda^{\prime}$ has $k$ parts. Hence conjugation yields bijections $P_{k}(n) \rightarrow P^{k}(n)$ and $P_{\leq k}(n) \rightarrow P^{\leq k}(n)$.

b) Let $P_{\mathrm{sc}}(n):=\left\{\lambda \in P(n) ; \lambda=\lambda^{\prime}\right\}$ be the set of self-conjugate partitions of $n$. We proceed to determine their number: To this end, given any $\lambda \vdash n$, let $r(\lambda):=\max \left\{i \in \mathbb{N} ; \lambda_{i} \geq i\right\} \in \mathbb{N}$ for $n \geq 1$, and $r([]):=0$, be the rank of $\lambda$; note that the largest square fitting into the upper left hand corner of the Young diagram of $\lambda$ has edge length $r(\lambda)$, it is called the Durfee square of $\lambda$. Moreover, let $h_{i}(\lambda):=\left(\lambda_{i}-i\right)+\left(\lambda_{i}^{\prime}-i\right)+1 \in \mathbb{N}$, for $i \in\{1, \ldots, r(\lambda)\}$, be the associated (diagonal) hook lengths; note that $h_{i}(\lambda)$ is the length of the hook centered at the $i$-th box on main diagonal of the Young diagram of $\lambda$, where $\lambda_{i}-i \in \mathbb{N}_{0}$ and $\lambda_{i}^{\prime}-i \in \mathbb{N}_{0}$ are the associated arm and leg lengths, respectively. Hence we get the partition $h(\lambda):=\left[h_{1}(\lambda), \ldots, h_{r(\lambda)}(\lambda)\right]$ of $n$, consisting of $r(\lambda)$ pairwise distinct parts, giving rise to the map $P(n) \rightarrow D(n): \lambda \mapsto h(\lambda)$.

If $\lambda \in P_{\mathrm{sc}}(n)$, then $h(\lambda)=\left[2\left(\lambda_{i}-i\right)+1 \in \mathbb{N} ; i \in\{1, \ldots, r(\lambda)\}\right]$ consists of odd parts, that is $h(\lambda) \in O_{r(\lambda)}(n) \cap D_{r(\lambda)}(n)=C_{r(\lambda)}(n)$, and thus we get the restriction $P_{\mathrm{sc}}(n) \rightarrow C(n): \lambda \mapsto h(\lambda)$, which moreover is injective. We show surjectivity: Given $\mu=\left[\mu_{1}, \ldots, \mu_{n}\right] \in C(n)$, let $\lambda_{i}:=\frac{\mu_{i}-1}{2}+i$ for $i \in$ $\{1, \ldots, l(\mu)\}$, and $\lambda_{i}:=\left|\left\{j \in\{1, \ldots, l(\mu)\} ; \lambda_{j} \geq i\right\}\right|$ for $i>l(\mu)$. Then, since $\mu_{i}-\mu_{i+1} \geq 2$, for all $i \in\{1, \ldots, l(\mu)-1\}$, we have $\lambda_{1} \geq \lambda_{2} \geq \cdots \geq \lambda_{l(\mu)} \geq$ $l(\mu) \geq \lambda_{l(\mu)+1} \geq \lambda_{l(\mu)+2} \geq \cdots \geq 0$, and from $\sum_{i=1}^{l(\mu)} \lambda_{i}=\frac{1}{2} \cdot \sum_{i=1}^{l(\mu)} \mu_{i}-\frac{l(\mu)}{2}+$ $\left(\begin{array}{c}l(\mu)+1 \\ 2\end{array}\right)=\frac{n}{2}+\frac{l(\mu)^{2}}{2}$ and $\sum_{i>l(\mu)} \lambda_{i}=\sum_{i>l(\mu)}\left|\left\{j \in\{1, \ldots, l(\mu)\} ; \lambda_{j} \geq i\right\}\right|=$ $\sum_{j=1}^{l(\mu)}\left|\left\{i>l(\mu) ; i \leq \lambda_{j}\right\}\right|=\sum_{j=1}^{l(\mu)}\left(\lambda_{j}-l(\mu)\right)=\sum_{j=1}^{l(\mu)} \lambda_{j}-l(\mu)^{2}$ we get $\sum_{i \geq 1} \lambda_{i}=$ $2 \cdot \sum_{i=1}^{l(\mu)} \lambda_{i}-l(\mu)^{2}=n$. Hence $\lambda:=\left[\lambda_{1}, \ldots, \lambda_{n}\right]$ is a partition of $n$, such that $r(\lambda)=l(\mu)$, which by construction is self-conjugate such that $h(\lambda)=\mu$.

Thus we conclude $P_{\mathrm{sc}}:=\sum_{n \geq 0}\left|P_{\mathrm{sc}}(n)\right| X^{n}=c=\prod_{i \geq 1}\left(1+X^{2 i-1}\right) \in \mathbb{Q}[[X]]$.

(14.4) Euler's identity. We reconsider the weighted generating series $D=$ $\sum_{n \geq 0}\left(\sum_{k=0}^{n}\left|D_{k}(n)\right| Y^{k}\right) X^{n}=\prod_{i \geq 1}\left(1+Y X^{i}\right) \in \mathbb{Q}[Y][[X]]$. Thus evaluating 
at $y:=-1$ yields the identity $\sum_{n \geq 0}\left(\left|D_{\text {even }}(n)\right|-\left|D_{\text {odd }}(n)\right|\right) X^{n}=\prod_{i \geq 1}(1-$ $\left.X^{i}\right) \in \mathbb{Q}[[X]]$, where $D_{\text {even }}(n):=\{\bar{\lambda} \in D(n) ; l(\lambda)$ even $\}$ and $D_{\text {odd }}(n):=\{\lambda \in$ $D(n) ; l(\lambda)$ odd $\}$ are the sets of distinct-part partitions of $n \in \mathbb{N}_{0}$ having an even respectively an odd number of parts; note that $\prod_{i \geq 1}\left(1-X^{i}\right)=p^{-1} \in \mathbb{Q}[[X]]$.

We proceed to show the pentagonal number theorem, saying that $d_{n}:=$ $\left|D_{\text {even }}(n)\right|-\left|D_{\text {odd }}(n)\right| \neq 0$ only if $n \in\{0\} \dot{\cup}\left\{n_{k}^{ \pm} \in \mathbb{N} ; k \in \mathbb{N}\right\}$, where $n_{k}^{ \pm}:=$ $\frac{1}{2} k(3 k \pm 1) \in \mathbb{N}$, and that $d_{n_{k}^{ \pm}}=(-1)^{k}$; a few examples are given in Table 12. The relevant numbers are $n^{+}=[2,7,15,26,40,57,77,100,126,155, \ldots]$ and $n^{-}=[1,5,12,22,35,51,70,92,117,145, \ldots]$, where the latter sequence explains the terminology inasmuch this is the number of vertices in a configuration of nested 5-gons; note that the numbers $n_{k}^{ \pm}$, for $k \in \mathbb{N}$, are pairwise distinct.

We consider the following surgical procedure on partitions, leading to an essentially sign-reversing involution on the distinct-part ones: Given a partition $\lambda=\left[\lambda_{1}, \ldots, \lambda_{k}\right]$ of $n \in \mathbb{N}$ having $k \in \mathbb{N}$ parts, then $\lambda_{k}$ is the number of boxes in the last row of the Young diagram of $\lambda$, and $d=d(\lambda):=\max \{i \in$ $\left.\{1, \ldots, k\} ; \lambda_{i}=\lambda_{1}-i+1\right\} \in \mathbb{N}$ is the length of the maximal anti-diagonal at its upper right hand corner. Now let $\lambda \in D(n)$, and we distinguish two cases:

Firstly, if $d<\lambda_{k}$ we obtain $\mu=\left[\lambda_{1}-1, \ldots, \lambda_{d}-1, \lambda_{d+1}, \ldots, \lambda_{k}, d\right]$, whose Young diagram is obtained from that of $\lambda$ by removing the maximal anti-diagonal and gluing a row of the same length underneath. Thus, if $d<k$ then by definition of $d$ we have $\lambda_{d}-1>\lambda_{d+1}$, and if $d=k$ then we have $\lambda_{d}-1=\lambda_{k}-1 \geq d$. Hence $\mu$ is a partition of $n$, having $k+1$ parts such that $d(\mu) \geq d=\mu_{k+1}$. Moreover, $\mu$ has pairwise distinct parts, except we have $d=k$ and $\lambda_{k}=k+1$, which happens if and only if $\lambda=\lambda^{k,+}:=[2 k, 2 k-1, \ldots, k+1] \vdash \frac{1}{2} k(3 k+1)=n_{k}^{+}$. For example, for $\lambda:=[8,7,5,3] \vdash 23$ we get $\mu:=[7,6,5,3,2] \vdash 23$, but for $\lambda:=[8,7,6,5] \vdash 26$ we get $\mu:=[7,6,5,4,4] \vdash 26$.

Secondly, if $c:=\lambda_{k} \leq d$ we aim at reversing this procedure, by removing the last row of the Young diagram of $\lambda$ and gluing an anti-diagonal of the same length to its upper right hand corner. This works well if $c<k$, yielding $\mu=\left[\lambda_{1}+1, \ldots, \lambda_{c}+1, \lambda_{c+1}, \ldots, \lambda_{d}, \ldots, \lambda_{k-1}\right]$, which is a partition of $n$, having $k-1$ pairwise distinct parts such that $d(\mu)=c=\lambda_{k}<\lambda_{k-1}=\mu_{k-1}$. But if $c=k$ the gluing step fails, yielding $\mu=\left[\lambda_{1}+1, \ldots, \lambda_{c-1}+1,1\right]$, which is a partition of $n$ having $k$ distinct parts. From $c=\lambda_{k} \leq d \leq k$ we infer that this occurs if and only if $\lambda=\lambda^{k,-}:=[2 k-1,2 k-2, \ldots, k] \vdash \frac{1}{2} k(3 k-1)=n_{k}^{-}$. For example, for $\lambda:=[7,6,5,3,2] \vdash 23$ we get $\mu:=[8,7,5,3] \vdash 23$, but for $\lambda:=[7,6,5,4] \vdash 22$ we get $\mu:=[8,7,6,1] \vdash 22$.

Thus letting $D_{\text {exc }}:=\left\{\lambda^{k, \pm} \in D_{k}\left(n_{k}^{ \pm}\right) ; k \in \mathbb{N}\right\}$ this defines an involutory map on $D(n) \backslash D_{\text {exc }}$, where $n \in \mathbb{N}$, which changes the length of partitions by \pm 1 . Hence if $n \notin\left\{n_{k}^{ \pm} \in \mathbb{N} ; k \in \mathbb{N}\right\}$ we get $d_{n}=0$, while for $n=n_{k}^{ \pm}$we get $d_{n}=(-1)^{k}$. Hence we have proved the Euler identity $\prod_{i \geq 1}\left(1-X^{i}\right)=$ $1+\sum_{k \geq 1}(-1)^{k}\left(X^{\frac{1}{2} k(3 k-1)}+X^{\frac{1}{2} k(3 k+1)}\right) \in \mathbb{Q}[[X]]$.

Recalling that $\prod_{i \geq 1}\left(1-X^{i}\right)=p^{-1} \in \mathbb{Q}[[X]]$, we get $\left(\sum_{n \geq 0} p_{n} X^{n}\right) \cdot(1+$ 
Table 12: Distinct-part partitions.

\begin{tabular}{|r||l|l|r|}
\hline$n$ & $D_{\text {even }}(n)$ & $D_{\text {odd }}(n)$ & $d_{n}$ \\
\hline \hline 0 & {[} & & 1 \\
1 & & {$[1]$} & -1 \\
2 & & {$[2]$} & -1 \\
3 & {$[2,1]$} & {$[3]$} &. \\
4 & {$[3,1]$} & {$[4]$} &. \\
5 & {$[4,1],[3,2]$} & {$[5]$} & 1 \\
6 & {$[5,1],[4,2]$} & {$[6],[3,2,1]$} &. \\
7 & {$[6,1],[5,2],[4,3]$} & {$[7],[4,2,1]$} & 1 \\
8 & {$[7,1],[6,2],[5,3]$} & {$[8],[5,2,1],[4,3,1]$} &. \\
\hline
\end{tabular}

$\left.\sum_{k \geq 1}(-1)^{k}\left(X^{\frac{1}{2} k(3 k-1)}+X^{\frac{1}{2} k(3 k+1)}\right)\right)=1 \in \mathbb{Q}[[X]]$, and thus convolutional multiplication for $n \in \mathbb{N}$ says $p_{n}=\sum_{k \geq 1}(-1)^{k-1}\left(p_{n-n_{k}^{+}}+p_{n-n_{k}^{-}}\right)$, where we agree on letting $p_{i}:=0$ for $i<0$. Actually, this is the most efficient tool known to determine all partition numbers $p_{1}, \ldots, p_{n}$ at the same time.

\section{Group actions}

\section{Actions}

(15.1) Actions. a) Let $G$ be a group, and let $N$ be a set. Then $G$ is said to act on the $G$-set $N$, if there is an action map $G \times N \rightarrow N:[g, x] \mapsto g x$ such that $1 x=x$, and $(g h) x=g(h x)$, for all $g, h \in G$ and $x \in N$. If $M$ and $N$ are $G$-sets, then a map $\alpha: M \rightarrow N$ such that $\alpha(g x)=g \cdot \alpha(x)$, for all $g \in G$ and $x \in M$, is called a homomorphism of $G$-sets; a bijective homomorphism is called an isomorphism, and isomorphic $G$-sets are called equivalent.

Given an action of $G$ on $N$, for $g \in G$ let $\varphi_{g}: N \rightarrow N: x \mapsto g x$. Hence from $\varphi_{g} \varphi_{g^{-1}}=\varphi_{g^{-1}} \varphi_{g}=\varphi_{1}=\operatorname{id}_{N}$ we get $\varphi_{g} \in \mathcal{S}_{N}:=\operatorname{Bij}(N, N)$, for all $g \in G$, and since $\varphi_{g} \varphi_{h}=\varphi_{g h}$, for all $g, h \in G$, we have an action homomorphism $G \rightarrow$ $\mathcal{S}_{N}: g \mapsto \varphi_{g}$. Conversely, if $\varphi: G \rightarrow \mathcal{S}_{N}: g \mapsto \varphi_{g}$ is a group homomorphism, then $G \times N \rightarrow N:[g, x] \mapsto \varphi_{g}(x)$ is an action of $G$ on $N$ : We have $\varphi_{1}=$ $\operatorname{id}_{N} \in \mathcal{S}_{N}$, and $\varphi_{g} \varphi_{h}=\varphi_{g h}$ implies $(g h) x=g(h x)$ for all $g, h \in G$ and $x \in N$. Moreover, if $\alpha: N \rightarrow N$ is an isomorphism of $G$-sets, then the action $\psi_{g} \in \mathcal{S}_{N}$ of $g \in G$ on $N$ is given as $\psi_{g}=\alpha \varphi_{g} \alpha^{-1}$.

Any group $G$ acts trivially on any set $N$ by letting $\varphi_{g}=\operatorname{id}_{N}$ for all $g \in G$. If $G$ acts faithfully on the set $N$, that is the action homomorphism $\varphi: G \rightarrow \mathcal{S}_{N}$ is injective, then $G$ is called a permutation group on $N$, and can be identified with a subgroup of $\mathcal{S}_{N}$. In particular, $\mathcal{S}_{N}$ acts naturally on $N$ by $\varphi_{\pi}: N \rightarrow$ 
$N: i \mapsto \pi(i)$, for all $\pi \in \mathcal{S}_{N}$, the action homomorphism being $\operatorname{id}_{\mathcal{S}_{N}}$.

If $N$ is a finite $G$-set, then for any $g \in G$ we get a permutation $\varphi_{g} \in \mathcal{S}_{N}$, and thus a cycle type $\lambda(g)=\lambda_{\varphi}(g):=\lambda\left(\varphi_{g}\right)$, a partition of $|N|$. If $\psi: G \rightarrow \mathcal{S}_{N}$ is an equivalent action induced by $\alpha \in \mathcal{S}_{N}$, that is we have $\psi_{g}=\alpha \varphi_{g} \alpha^{-1}$ for all $g \in G$, then we have $\lambda_{\psi}(g)=\lambda\left(\psi_{g}\right)=\lambda\left(\varphi_{g}\right)=\lambda_{\varphi}(g)$ for all $g \in G$, that is the cycle type of $g$ only depends on the equivalence class of $G$-actions considered.

(15.2) Orbits. Let $G$ be a group, and let $N$ be a $G$-set. The relation $\mathcal{R}:=$ $\{[x, y] \in N \times N ; y=g x$ for some $g \in G\}$ is an equivalence relation on $N$ : From $1 x=x$ we infer that $\mathcal{R}$ is reflexive; from $y=g x$ we get $g^{-1} y=x$, thus $\mathcal{R}$ is symmetric; and from $y=g x$ and $z=h y$ we get $z=h g x$, thus $\mathcal{R}$ is transitive.

Given $x \in N$, its equivalence class $G x:=\{g x \in N ; g \in G\}$ again is a $G$-set, called the $G$-orbit of $x$; its cardinality $|G x|$ is called its length, and a subset $T \subseteq G$ such that $T \rightarrow G x: t \mapsto t x$ is a bijection is called a transversal of $G x$ with respect to $x$. Let $G \backslash N:=\{G x \subseteq N ; x \in N\}$ be the set of $G$-orbits; a subset $S \subseteq N$ such that $S \rightarrow G \backslash N: x \mapsto G x$ is a bijection is called a set of orbit representatives of $N$, hence we have $N=\coprod_{x \in S} G x$. If $N \neq \emptyset$ and $N=G x$ for any and thus all $x \in N$, then $N$ is called a transitive $G$-set. Note that transversals and orbit representatives always exist by the Axiom of Choice.

For $x \in N$ let $G_{x}=\operatorname{Stab}_{G}(x):=\{g \in G ; g x=x\} \leq G$, being called the stabilizer of $x$ in $G$ : We have $1 \in G_{x}$, and for $g, h \in G_{x}$ we have $g^{-1} h x=x$, hence $g^{-1} h \in G_{x}$ as well. Moreover, the stabilizers of elements in the same orbit are conjugate in $G$, more precisely for $g \in G$ we have $G_{g x}=g G_{x} g^{-1}$ : For $h \in G_{x}$ we have $g h g^{-1} \cdot g x=g x$, hence $g G_{x} g^{-1} \leq G_{g x}$, thus we also have $g^{-1} G_{g x} g \leq G_{g^{-1} g x}=G_{x}$, implying $G_{g x} \leq g G_{x} g^{-1}$.

For example, for the trivial $G$-set the orbits are the singleton subsets of $N$, and we have $G_{x}=G$ for all $x \in N$. Moreover, the natural action of $\mathcal{S}_{n}$, where $n \in \mathbb{N}$, is transitive such that $\operatorname{Stab}_{\mathcal{S}_{n}}(n)=\mathcal{S}_{n-1}$; and for any $\pi \in \mathcal{S}_{n}$ the orbits of $\langle\pi\rangle \leq \mathcal{S}_{n}$ in the natural action are just the cycles of $\pi$.

(15.3) Dihedral groups. Let $\mathbb{R}^{2 \times 1}$ be the Euclidean plane equipped with the standard scalar product, and let $\mathrm{GO}_{2}(\mathbb{R}):=\left\{g \in \mathrm{GL}_{2}(\mathbb{R}) ; g g^{\mathrm{tr}}=E_{2}\right\} \leq$ $\mathrm{GL}_{2}(\mathbb{R})$ be the associated orthogonal group. We have $\mathrm{GO}_{2}(\mathbb{R})=\{g \in$ $\left.\mathrm{GO}_{2}(\mathbb{R}) ; \operatorname{det}(g)=1\right\} \dot{\cup}\left\{g \in \mathrm{GO}_{2}(\mathbb{R}) ; \operatorname{det}(g)=-1\right\}$, where the elements of the special orthogonal group $\mathrm{SO}_{2}(\mathbb{R}):=\left\{g \in \mathrm{GO}_{2}(\mathbb{R}) ; \operatorname{det}(g)=1\right\} \leq \mathrm{GO}_{2}(\mathbb{R})$ are called rotations, while those of $\mathrm{GO}_{2}(\mathbb{R}) \backslash \mathrm{SO}_{2}(\mathbb{R})$ are called reflections.

For $n \geq 3$ let $\mathcal{R}_{n} \subseteq \mathbb{R}^{2 \times 1}$ be a regular $n$-gon centered at the origin, and let $G:=$ $\left\{g \in \mathrm{GO}_{2}(\mathbb{R}) ; g\left(\mathcal{R}_{n}\right)=\mathcal{R}_{n}\right\}$ be its group of symmetries; then $G \cap \mathrm{SO}_{2}(\mathbb{R})$ is called its group of rotations. Hence $G$ acts transitively on the $n$ vertices of $\mathcal{R}_{n}$, thus numbering them counterclockwise yields an action homomorphism $\varphi: G \rightarrow \mathcal{S}_{n}$, which since the vertices contain an $\mathbb{R}$-basis of $\mathbb{R}^{2 \times 1}$ is injective; the image $D_{2 n}:=\operatorname{im}(\varphi) \leq \mathcal{S}_{n}$ is called the associated dihedral group.

We describe the elements of $D_{2 n}$, showing that $\left|D_{2 n}\right|=2 n$; see Table 13 for 
Table 13: Dihedral groups $D_{6}$ and $D_{8}$.

\begin{tabular}{|l|l|l|}
\hline$\pi \in D_{6}$ & $\lambda(\pi)$ & $k(\pi)$ \\
\hline \hline() & {$\left[1^{3}\right]$} & 3 \\
$(1,2)$ & {$[2,1]$} & 2 \\
$(1,3)$ & {$[2,1]$} & 2 \\
$(2,3)$ & {$[2,1]$} & 2 \\
$(1,2,3)$ & {$[3]$} & 1 \\
$(1,3,2)$ & {$[3]$} & 1 \\
\hline
\end{tabular}

\begin{tabular}{|l|l|l|}
\hline$\pi \in D_{8}$ & $\lambda(\pi)$ & $k(\pi)$ \\
\hline \hline() & {$\left[1^{4}\right]$} & 4 \\
$(1,3)$ & {$\left[2,1^{2}\right]$} & 3 \\
$(2,4)$ & {$\left[2,1^{2}\right]$} & 3 \\
$(1,2)(3,4)$ & {$\left[2^{2}\right]$} & 2 \\
$(1,3)(2,4)$ & {$\left[2^{2}\right]$} & 2 \\
$(1,4)(2,3)$ & {$\left[2^{2}\right]$} & 2 \\
$(1,2,3,4)$ & {$[4]$} & 1 \\
$(1,4,3,2)$ & {$[4]$} & 1 \\
\hline
\end{tabular}

$D_{6}=\mathcal{S}_{3}$ and $D_{8}$ : Since rotations in $\mathrm{GO}_{2}(\mathbb{R})$ are determined by their rotation angle, the rotations in $D_{2 n}$ are those with angle $\frac{2 k \pi}{n}$, for $k \in\{0, \ldots, n-1\}$. Thus $D_{2 n}$ contains precisely $n$ rotations, given as $\left\langle\tau_{n}\right\rangle=\left\{\tau_{n}^{k} \in \mathcal{S}_{n} ; k \in\{0, \ldots, n-1\}\right\}$, where $\tau_{n}:=(1,2, \ldots, n) \in \mathcal{S}_{n}$. Since reflections in $\mathrm{GO}_{2}(\mathbb{R})$ are determined by their reflection axis, we distinguish the cases $n$ odd and $n$ even:

For $n$ odd the axis runs through one of the vertices of $\mathcal{R}_{n}$ and the edge opposite; thus there are precisely $n$ reflections, one of them being $\sigma_{n}:=(2, n)(3, n-$ 1) $\cdots\left(\frac{n+1}{2}, \frac{n+3}{2}\right) \in \mathcal{S}_{n}$. For $n$ even the axis either runs through a pair of opposite vertices, or runs through a pair of opposite edges; thus again there are precisely $\frac{n}{2}+\frac{n}{2}=n$ reflections, one of the former being $\sigma_{n}:=(2, n)(3, n-1) \cdots\left(\frac{n}{2}, \frac{n+4}{2}\right) \in$ $\mathcal{S}_{n}$ and one of the latter being $(1,2)(3, n)(4, n-1) \cdots\left(\frac{n+2}{2}, \frac{n+4}{2}\right) \in \mathcal{S}_{n}$. In both cases we have $\operatorname{Stab}_{D_{2 n}}(1)=\left\langle\sigma_{n}\right\rangle$.

(15.4) Cosets. a) Let $G$ be a group, and $U \leq G$. Then $U$ acts on $G$ by right multiplication $\rho_{u}: G \rightarrow G: x \mapsto x u^{-1}$, for all $u \in U$ : We have $\rho_{1}(x)=x 1^{-1}=$ $x$ and $\rho_{u v}(x)=x(u v)^{-1}=x v^{-1} u^{-1}=\rho_{u}\left(\rho_{v}(x)\right)$, for all $x \in G$ and $u, v \in U$. Hence the $U$-orbit of $x \in G$ is the (left) coset $x U:=\{x u \in G ; u \in U\} \subseteq G$.

The group $G$ acts transitively on $G / U:=\{x U \subseteq G ; x \in G\}$ by left multiplication $\lambda_{g}: G / U \rightarrow G / U: x U \mapsto g x U$, for all $g \in G$ : We have $1(x U)=x U$ and $g h(x U)=g(h x U)$, for all $g, h, x \in G$, and $x U=x(1 \cdot U)$. Since $g u \in U$ if and only if $g \in U$, for $g \in G$, we have $G_{U}=U$. A transversal $T \subseteq G$ of the action on $G / U$ is called a (left) transversal of $U$ in $G$; then we have $G=\coprod_{t \in T} t U$, and $[G: U]:=|G / U|=|T| \in \mathbb{N} \dot{\cup}\{\infty\}$ is called the index of $U$ in $G$.

b) Let $N$ be a transitive $G$-set, and $x \in N$. Then $\alpha: G / G_{x} \rightarrow N: g G_{x} \mapsto g x$ is an isomorphism of $G$-sets: For $g \in G$ and $u \in G_{x}$ we have $g u x=g x$, hence $\alpha$ is well-defined. Since $N$ is transitive we infer that $\alpha$ is surjective. For $g, h \in G$ such that $g x=h x$ we have $h^{-1} g \in G_{x}$, that is $g \in h G_{x}$, thus $\alpha$ is injective. Finally, we have $\alpha\left(g h G_{x}\right)=g h x=g(h x)=g \cdot \alpha\left(h G_{x}\right)$, for all $g, h \in G$.

c) Let now $G$ be finite, and let $U \leq G$. Then we have $[G: U]=\frac{|G|}{|U|}$ : Let $T \subseteq G$ 
be a transversal of $U$ in $G$, hence $G=\coprod_{t \in T} t U$. For the $U$-orbit $t U$ we have $U_{t}=\left\{u \in U ; t u^{-1}=t\right\}=\{1\}$. Hence $U \rightarrow U /\{1\} \rightarrow t U: u \mapsto u\{1\} \mapsto t u^{-1}$ is a bijection, thus $|t U|=|U|$ and $|G|=|T| \cdot|U|$.

In particular, we have Lagrange's Theorem saying that $|U||| G \mid$. Moreover, for $U:=G_{x} \leq G$, where $x \in N$, this implies the orbit theorem saying that $|N|=\left[G: G_{x}\right]=\frac{|G|}{\left|G_{x}\right|}$; in particular we have $|N||| G \mid$.

(15.5) The Cauchy-Frobenius-Burnside Lemma. a) Let $G$ be a finite group, let $N$ be a finite $G$-set, and for $g \in G$ let $\operatorname{Fix}_{N}(g):=\{x \in N ; g x=x\}$ be its set of fixed points. Then we have the Cauchy-Frobenius-Burnside Lemma $|G \backslash N|=\frac{1}{|G|} \cdot \sum_{g \in G}\left|\operatorname{Fix}_{N}(g)\right|$; note that $|G \backslash N|$ and $\left|\operatorname{Fix}_{N}(g)\right|$ only depend on the equivalence class of $G$-actions considered:

Letting $\mathcal{A}:=\{[g, x] \in G \times N ; g x=x\}$ we use double counting to determine $|\mathcal{A}|$ in two ways: On the one hand we have $|\mathcal{A}|=\sum_{g \in G}|\{x \in N ; g x=x\}|=$ $\sum_{g \in G}\left|\operatorname{Fix}_{N}(g)\right|$. On the other hand we have $|\mathcal{A}|=\sum_{x \in N}|\{g \in G ; g x=x\}|=$ $\sum_{x \in N}\left|G_{x}\right|$. For $y \in G x$, where $x \in N$, we have $|G y|=|G x|$, and thus $\left|G_{x}\right|=$ $\left|G_{y}\right|$. Letting $S \subseteq N$ be a set of orbit representatives, we get $\sum_{x \in N}\left|G_{x}\right|=$ $\sum_{x \in S} \sum_{y \in G x}\left|G_{y}\right|=\sum_{x \in S}|G x| \cdot\left|G_{x}\right|=\sum_{x \in S}|G|=|G \backslash N| \cdot|G|$.

Note that enumerating $G=\left\{g_{1}, g_{2}, \ldots\right\}$ and $N=\left\{x_{1}, x_{2}, \ldots\right\}$, the set $\mathcal{A}$ can be represented by the indicator matrix $A:=\left[a_{i j}\right]_{i j} \in\{0,1\}^{|G| \times|N|}$, where $a_{i j}:=1$ if $g_{i} x_{j}=x_{j}$, and $a_{i j}:=0$ if $g_{i} x_{j} \neq x_{j}$. Hence $|\mathcal{A}|$ is the sum of the entries of $A$, whose $i$-th row sum equals $\left|\operatorname{Fix}_{N}\left(g_{i}\right)\right|$ and whose $j$-th column sum equals $\left|G_{x_{j}}\right|$. The two ways of counting are then computing the sum of the rows sums, and computing the sum of the column sums, respectively.

b) The group $G$ acts diagonally on $N \times N$ by $g$ : $[y, z] \mapsto[g y, g z]$, for all $g \in G$. Note that the diagonal $\{[y, y] \in N \times N ; y \in N\}$ is a union of orbits, and consists of a single orbit if and only if $G$ acts transitively.

If $G$ acts transitively on $N$, then for all $x \in N$ the map $G_{x} \backslash N \rightarrow G \backslash(N \times$ $N): G_{x} y \mapsto G[x, y]$ is a bijection, and we have $\left|G_{x} \backslash N\right|=|G \backslash(N \times N)|=$ $\frac{1}{|G|} \cdot \sum_{g \in G}\left|\operatorname{Fix}_{N}(g)\right|^{2}$, being called the rank of $N$ :

Let $z \in G_{x} y$, then there is $g \in G_{x}$ such that $z=g y$, and hence we have $[x, z]=[x, g y]=[g x, g y]=g[x, y] \in G[x, y]$, implying that the above map is well-defined. Because of transitivity, for any $z \in N$ there is $g \in G$ such that $g z=x$, and hence we have $G[z, y]=G[x, g y]$, showing that the above map is surjective. Finally, letting $z \in N$ such that $G[x, y]=G[x, z]$, that is there is $g \in G$ such that $[x, z]=g[x, y]=[g x, g y]$, we infer that $g \in G_{x}$, hence $z \in G_{x} y$, implying injectivity. Finally we have $\operatorname{Fix}_{N \times N}(g)=\operatorname{Fix}_{N}(g) \times \operatorname{Fix}_{N}(g)$.

(15.6) Fixed-point-free elements. a) Let $G$ act transitively on $N$, where $n:=|N| \in \mathbb{N}$ and $r:=|G \backslash(N \times N)| \in \mathbb{N}$, and let $\mathcal{D}=\mathcal{D}_{0}(G, N):=\{g \in$ $\left.G ; \operatorname{Fix}_{N}(g)=\emptyset\right\} \subseteq G$ be the set fixed-point-free elements. Letting $x \in N$ we have the Cameron-Cohen inequality [1992] saying $|\mathcal{D}| \geq(r-1) \cdot\left|G_{x}\right| \geq n-1$ : 
We have $|G|=\sum_{g \in G}\left|\operatorname{Fix}_{N}(g)\right|=\sum_{g \in G \backslash \mathcal{D}}\left|\operatorname{Fix}_{N}(g)\right|$, as well as $(r-1) \cdot\left|G_{x}\right|=$ $\sum_{g \in G_{x}}\left|\operatorname{Fix}_{N \backslash\{x\}}(g)\right|$, where $G_{x} \cap \mathcal{D}=\emptyset$ anyway. This yields $|G|-(r-1) \cdot\left|G_{x}\right|=$ $\sum_{g \in G_{x}}\left(\left|\operatorname{Fix}_{N}(g)\right|-\left|\operatorname{Fix}_{N \backslash\{x\}}(g)\right|\right)+\sum_{g \in G \backslash\left(G_{x} \cup \mathcal{D}\right)}\left|\operatorname{Fix}_{N}(g)\right|$. Since $\left|\operatorname{Fix}_{N}(g)\right|-$ $\left|\operatorname{Fix}_{N \backslash\{x\}}(g)\right|=1$ for all $g \in G_{x}$, and $\left|\operatorname{Fix}_{N}(g)\right| \geq 1$ for all $g \in G \backslash \mathcal{D}$, we infer $|G|-(r-1) \cdot\left|G_{x}\right| \geq|G \backslash \mathcal{D}|=|G|-|\mathcal{D}|$. Moreover, since any of the $r-1$ orbits of $G_{x}$ on $N \backslash\{x\}$ has length at most $\left|G_{x}\right|$, we get $(r-1) \cdot\left|G_{x}\right| \geq n-1$. $\sharp$

Thus for $n \geq 2$, that is $r \geq 2$, we have the Lenstra inequality [1991] saying $\frac{|\mathcal{D}|}{|G|} \geq \frac{(r-1) \cdot\left|G_{x}\right|}{n \cdot\left|G_{x}\right|}=\frac{(r-1)}{n} \geq \frac{1}{n}$, only depending on $n$, but being independent of $|G|$; In particular, this also implies Jordan's Theorem [1872] saying that for $n \geq 2$ we have $\mathcal{D} \neq \emptyset$.

b) Assuming that $G$ acts faithfully, we determine the cases where the Lenstra inequality actually is an equality; in particular, it will turn out that then $n$ is a prime power, and that for any such $n$ there actually is a group achieving equality:

Now $\frac{|\mathcal{D}|}{|G|}=\frac{1}{n}$ implies $r=2$, that is $G$ acts transitively on the pairs of distinct elements of $N$, in other words $G$ acts 2-fold transitively on $N$. Moreover, we have equality $|\mathcal{D}|=\left|G_{x}\right|=(r-1) \cdot\left|G_{x}\right|$, from which the estimates in the proof of the Cameron-Cohen inequality yield $\left|\operatorname{Fix}_{N}(g)\right|=1$ for all $g \in G \backslash\left(G_{x} \cup \mathcal{D}\right)$, that is any such $g$ is contained in $G_{y}$ for some unique $y \in N \backslash\{x\}$. Since any $1 \neq g \in G$ acts non-trivially, we conclude that $G=\{1\} \dot{\cup} \mathcal{D} \cup \coprod_{x \in N}\left(G_{x} \backslash\{1\}\right)$. Hence we have $G_{x, y}=G_{x} \cap G_{y}=\{1\}$ for all $x \neq y \in N$, that is $G$ acts sharply 2-fold transitively on $N$, and thus $\left|G_{x}\right|=|\mathcal{D}|=n-1$ and $|G|=n \cdot\left|G_{x}\right|=n(n-1)$.

Conversely, for any sharply 2-fold transitive group we have $\left|G_{x}\right|=n-1$ and $|G|=n(n-1)$, as well as a decomposition as above, so that $|\mathcal{D}|=|G|-1-$ $n \cdot\left(\left|G_{x}\right|-1\right)=n-1=\frac{|G|}{n}$. The sharply 2-fold transitive permutation groups have been classified by Zassenhaus [1936], using the notion of near-fields; we indicate the elementary steps, at least showing that $n$ is a prime power:

Let $g \in \mathcal{D}$. Since $g$ acts fixed point freely by conjugation on $\left\{G_{x} ; x \in N\right\}$, we infer that $g$ cannot possibly centralize any element of $\bigsqcup_{x \in N}\left(G_{x} \backslash\{1\}\right)$, which entails that $C_{G}(g) \subseteq\{1\} \cup \dot{\mathcal{D}}$. On the other hand, for any $x \in N$ the group $G_{x}$ acts on $\mathcal{D}$, where from $C_{G_{x}}(g)=C_{G}(g) \cap G_{x}=\{1\}$ and $\left|G_{x}\right|=|\mathcal{D}|$ we infer that $G_{x}$ acts regularly. Thus $\mathcal{D} \subseteq G$ is a single conjugacy class, and hence we have $\left|C_{G}(g)\right|=\frac{|G|}{|\mathcal{D}|}=n=|\mathcal{D}|+1$. In conclusion, we get $C_{G}(g)=\{1\} \dot{\cup} \mathcal{D}=: K$.

This shows that $K \unlhd G$ is a subgroup, which automatically is normal. (Note that this elegantly avoids the subtle theory of Frobenius groups.) From $|K|=n$ we get $G \cong K \rtimes G_{x}$, where since $K$ acts regularly on $N$ we may identify $K$ with $N$ via $K \rightarrow N: g \mapsto g x$. All elements of $\mathcal{D}$ being conjugate, we infer that these have one and the same order, which hence is a prime, $p$ say. Furthermore, $K$ is the centralizer of any element of $\mathcal{D}$, hence $K$ is abelian. Thus $K$ is an elementary abelian $p$-group, which we may write as $K \cong \mathbb{Z}_{p}^{d}$, for some $d \in \mathbb{N}$; in particular $n=p^{d}$. Using the above identification we may describe $G$ as an affine group $G \cong \mathbb{Z}_{p}^{d} \rtimes G_{0}$, where $G_{0} \leq \mathrm{GL}_{d}(p)$ is a subgroup of order $p^{d}-1$. 
The classification of sharply 2-fold transitive permutation groups now is given as follows, in particular showing that for any prime power there is such a group; see [5, Table 7.3]: i) Generically, $\mathbb{Z}_{p}^{d}$ can be identified with $\mathbb{F}_{p^{d}}^{+}$, where $p$ is a prime and $d \in \mathbb{N}$, and $G_{0} \leq \Gamma L_{1}\left(p^{d}\right)$ is a group of semilinear maps; note that $\left|\Gamma L_{1}\left(p^{d}\right)\right|=d \cdot\left(p^{d}-1\right)$, where $\mathrm{GL}_{1}\left(p^{d}\right)$ can be identified with a cyclic subgroup $T_{\text {Singer }} \leq \mathrm{GL}_{d}(p)$ of order $p^{d}-1$ generated by a Singer cycle, and $\Gamma L_{1}\left(p^{d}\right)$ can be identified with the normalizer of $T_{\text {Singer }}$ in $\mathrm{GL}_{d}(p)$. ii) Apart from that, $G$ is one of 7 exceptional cases, where $d=2$, and either $p \in\{5,7,11,23\}$ and $\mathrm{SL}_{2}(3) \leq G_{0}$, or $p \in\{11,19,29,59\}$ and $\mathrm{SL}_{2}(5) \leq G_{0}$; indeed, in the former cases $G_{0}$ has shape $\mathrm{SL}_{2}(3), \mathrm{SL}_{2}(3) \cdot 2, \mathrm{SL}_{2}(3) \times 5,\left(\mathrm{SL}_{2}(3) \cdot 2\right) \times 5$, while in the latter cases we get $\mathrm{SL}_{2}(5), \mathrm{SL}_{2}(5) \times 3, \mathrm{SL}_{2}(5) \times 7, \mathrm{SL}_{2}(5) \times 29$, respectively. $\sharp$

In particular, the above discussion implies that the estimate $\frac{|\mathcal{D}|}{|G|} \geq \frac{1}{n}$ is best possible, at least if the class of all permutation groups is considered. Since none of the sharply 2-transitive groups is simple, actually apart from finitely many exceptions they are all solvable, the question arises whether there are stronger bounds for certain classes of permutation groups, for example for simple groups acting primitively. Similarly, we might wonder whether there always are fixedpoint-free elements fulfilling further conditions, for example concerning their order. But all these kinds of questions are under ongoing discussion.

(15.7) The fixed point index. a) Let $G$ be a finite group, acting on $N:=$ $\{1, \ldots, n\}$, where $n \in \mathbb{N}_{0}$. For $k \in\{0, \ldots, n\}$ let $\mathcal{D}_{k}=\mathcal{D}_{k}(G, N):=\{g \in$ $\left.G ;\left|\operatorname{Fix}_{N}(g)\right|=k\right\}$, and $f_{k}=f_{k}(G, N):=\left|\mathcal{D}_{k}(G, N)\right| \in \mathbb{N}_{0}$. Then the polynomial $f_{G}=f_{G, N}:=\frac{1}{|G|} \cdot \sum_{k=0}^{n} f_{k} X^{k} \in \mathbb{Q}[X]$ is called the fixed point index of the action of $G$ on $N$; note that $f_{G}$ only depends on the equivalence class of $G$-actions considered. Hence the fixed point index has degree $n$ and leading coefficient $\frac{f_{n}}{|G|} \geq \frac{1}{|G|}$; note that $f_{n}=1$ if and only if $G$ acts faithfully.

Evaluating yields $f_{G}(1)=\frac{1}{|G|} \cdot \sum_{k=0}^{n} f_{k}=1$ and $f_{G}(0)=\frac{f_{0}}{|G|}$, where $f_{0}=\left|\mathcal{D}_{0}\right|$ is the number of the elements of $G$ acting fixed point freely. Evaluating the formal derivative $\frac{\partial}{\partial X} f_{G}=\frac{1}{|G|} \cdot \sum_{k=1}^{n} k f_{k} X^{k-1} \in \mathbb{Q}[X]$, the Cauchy-Frobenius-Burnside Lemma yields $\left(\frac{\partial}{\partial X} f_{G}\right)(1)=\frac{1}{|G|} \cdot \sum_{k=1}^{n} k f_{k}=\frac{1}{|G|} \cdot \sum_{k=0}^{n} k f_{k}=|G \backslash N|$. Moreover, if $G$ acts transitively, then for any $x \in N$ we have $\frac{\partial}{\partial X} f_{G, N}=f_{G_{x}, N \backslash\{x\}} \in \mathbb{Q}[X]$, the fixed point index of the action of $G_{x}$ on $N \backslash\{x\}$ :

For $k \geq 1$ we have $\mathcal{D}_{k}(G, N)=\bigcup_{y \in N} \mathcal{D}_{k-1}\left(G_{y}, N \backslash\{y\}\right)$, where any $g \in$ $\mathcal{D}_{k}(G, N)$ belongs to precisely $k$ of the $n$ sets $\mathcal{D}_{k-1}\left(G_{y}, N \backslash\{y\}\right)$. Stabilizers being $G$-conjugate we infer that $f_{k-1}\left(G_{y}, N \backslash\{y\}\right)=\left|\mathcal{D}_{k-1}\left(G_{y}, N \backslash\{y\}\right)\right|$ does not depend on $y \in N$. Thus we get $k \cdot f_{k}(G, N)=n \cdot f_{k-1}\left(G_{x}, N \backslash\{x\}\right)$. Using $|G|=n \cdot\left|G_{x}\right|$ this yields $\frac{\partial}{\partial X} f_{G, N}=\frac{1}{|G|} \cdot \sum_{k=1}^{n} k f_{k}(G, N) X^{k-1}=\frac{1}{\left|G_{x}\right|}$. $\sum_{k=1}^{n} f_{k-1}\left(G_{x}, N \backslash\{x\}\right) X^{k-1}=\frac{1}{\left|G_{x}\right|} \cdot \sum_{k=0}^{n-1} f_{k}\left(G_{x}, N \backslash\{x\}\right) X^{k}=f_{G_{x}, N \backslash\{x\}} \sharp$

b) For $k \in\{0, \ldots, n\}$, the group $G$ acts diagonally on $N^{k}$ by $g:\left[x_{1}, \ldots, x_{k}\right] \mapsto$ $\left[g x_{1}, \ldots, g x_{k}\right]$, for all $g \in G$. Thus the set $\mathcal{S}_{k}(N):=\operatorname{Inj}(\{1, \ldots, k\}, N)=$ $\left\{\left[x_{1}, \ldots, x_{k}\right] \in N^{k} ; x_{i} \neq x_{j}\right.$ for $\left.i \neq j\right\} \subseteq N^{k}$ of $k$-arrangements of $N$ is a union 
of orbits, hence also becomes a $G$-set; note that $\mathcal{S}_{0}(N)=N^{0}=\{[]\}$.

Letting $t_{k}=t_{k}(G, N):=\left|G \backslash \mathcal{S}_{k}(N)\right| \in \mathbb{N}_{0}$, the polynomial $t_{G}=t_{G, N}:=$ $\sum_{k=0}^{n} \frac{t_{k}}{k !} X^{k} \in \mathbb{Q}[X]$ is called the transitivity index of the action of $G$ on $N$; note that $t_{G}$ only depends on the equivalence class of $G$-actions considered. Note that we always have $t_{0}=1$, while $G$ acts transitively if and only if $t_{1}=1$. If $t_{k}=1$, for some $k \geq 1$, then $G$ is said to act $k$-fold transitively; note that in this case we have $t_{i}=1$ for all $i \in\{0, \ldots, k\}$.

Then we have Boston's Theorem [1993] $t_{G}(X)=f_{G}(X+1) \in \mathbb{Q}[X]$, that is $t_{G}=\tau f_{G} \in \mathbb{Q}[X]$, in terms of difference calculus: If $g \in \mathcal{D}_{j}$, for some $j \in\{0, \ldots, n\}$, then $g$ fixes precisely $\left|\operatorname{Inj}\left(\{1, \ldots, k\}, \operatorname{Fix}_{N}(g)\right)\right|=j_{(k)}$ elements of $\mathcal{S}_{k}(N)$. Hence the Cauchy-Frobenius-Burnside Lemma yields $t_{k}=$ $\frac{1}{|G|} \cdot \sum_{j=0}^{n} f_{j} j_{(k)}$, and thus $t_{G}(X)=\sum_{k=0}^{n} \frac{t_{k}}{k !} X^{k}=\frac{1}{|G|} \cdot \sum_{k=0}^{n} \sum_{j=0}^{n} \frac{f_{j} j_{(k)}}{k !} X^{k}=$ $\frac{1}{|G|} \cdot \sum_{j=0}^{n} f_{j}\left(\sum_{k=0}^{n}\left(\begin{array}{l}j \\ k\end{array}\right) X^{k}\right)=\frac{1}{|G|} \cdot \sum_{j=0}^{n} f_{j}(X+1)^{j}=f_{G}(X+1) \in \mathbb{Q}[X]$.

In particular, we have $\left(\left(\frac{\partial}{\partial X}\right)^{k} f_{G}\right)(1)=\left(\left(\frac{\partial}{\partial X}\right)^{k} t_{G}\right)(0)$, where in turn $\left(\frac{\partial}{\partial X}\right)^{k} t_{G}=$ $\left(\frac{\partial}{\partial X}\right)^{k}\left(\sum_{j=0}^{n} \frac{t_{j}}{j !} X^{j}\right)=\sum_{j=k}^{n} \frac{t_{j}}{(j-k) !} X^{j-k} \in \mathbb{Q}[X]$ yields $\left(\left(\frac{\partial}{\partial X}\right)^{k} t_{G}\right)(0)=t_{k}$, showing that $t_{G}$ can be easily computed from $f_{G}$.

c) For example, for the trivial group we get $f_{\{1\}}=X^{n} \in \mathbb{Q}[X]$. Hence, if $G$ acts regularly, then we have $G_{x}=\{1\}$, and formal integration yields $f_{G}=$ $\frac{1}{n} \cdot\left(X^{n}+(n-1)\right) \in \mathbb{Q}[X]$, reflecting the fact that $1_{G}$ is the only element $G$ having a fixed point. By the way, this also shows that the proportion of fixed-point-free elements of $G$ can indeed be as large as possible, namely $\frac{n-1}{n}$.

Iterating this, we obtain the fixed point indices of sharply $k$-transitive groups, for $k \in \mathbb{N}$, that is $G$ acts $k$-fold transitively such that $G_{n, n-1, \ldots, n-k+1}=\{1\}$; note that this is independent of the shape of the group considered. For example, for $n=12$ and $k=5$ we successively get, writing $f_{i}$ for the fixed point index of the stabilizer of an $i$-tuple, for $i \in\{0, \ldots, 5\}$ :

$$
\begin{aligned}
& f_{5}=X^{7} \\
& f_{4}=\frac{1}{8} \cdot\left(X^{8}+7\right) \\
& f_{3}=\frac{1}{72} \cdot\left(X^{9}+63 X+8\right) \\
& f_{2}=\frac{1}{720} \cdot\left(X^{10}+315 X^{2}+80 X+324\right), \\
& f_{1}=\frac{1}{7920} \cdot\left(X^{11}+1155 X^{3}+440 X^{2}+3564 X+2760\right), \\
& f_{0}=\frac{1}{95040} \cdot\left(X^{12}+3465 X^{4}+1760 X^{3}+21384 X^{2}+33120 X+35310\right)
\end{aligned}
$$

Prominent examples of the above situation are the sporadic simple Mathieu groups $M_{11}$ and $M_{12}$, acting sharply 4-transitive on 11 and sharply 5transitive on 12 points, respectively; see also (17.3). Hence from $f_{M_{11}}=f_{1}$ and $f_{M_{12}}=f_{0}$ we get the transitivity indices $t_{M_{11}}=f_{M_{11}}(X+1) \in \mathbb{Q}[X]$ and $t_{M_{12}}=f_{M_{12}}(X+1) \in \mathbb{Q}[X]$, and thus for $M_{11}$ we get $\left[t_{0}, \ldots, t_{11}\right]=$ $[1,1,1,1,1,7,42,210,840,2520,5040,5040]$ and for $M_{12}$ we get $\left[t_{0}, \ldots, t_{12}\right]=$ $[1,1,1,1,1,1,7,42,210,840,2520,5040,5040]$, reflecting the facts that $M_{11}$ acts 
4-fold, but not 5 -fold transitively, and that $M_{12}$ acts 5 -fold, but not 6 -fold transitively, respectively.

Formal integration might serve as a tool to proof the non-existence of transitive extensions: For example, for the natural action of the dihedral group $D_{8}$ on 4 points, by Table 13 we have $f_{D_{8}}=\frac{1}{8} \cdot\left(X^{8}+2 X^{2}+5\right) \in \mathbb{Q}[X]$, from which formal integration yields $f=\frac{1}{40} \cdot\left(X^{5}+\frac{10}{3} X^{3}+25 X+\frac{32}{3}\right) \in \mathbb{Q}[X]$, showing that there cannot be a group of order $5 \cdot 8=40$ acting transitively on 5 points, having point stabilizer $D_{8}$.

(15.8) Example: Derangements. For the natural action of the symmetric group $\mathcal{S}_{n}$ we get the following: Let $D_{n, k} \in \mathbb{N}_{0}$ be the number of permutations in $\mathcal{S}_{n}$ having precisely $k \in\{0, \ldots, n\}$ fixed points. Since $\mathcal{S}_{n}$ acts $n$-fold transitively on $N$, we have $t_{j}=1$ for all $j \in\{0, \ldots, n\}$, thus for the associated fixed point index we get $\frac{1}{n !} \cdot \sum_{k=0}^{n} D_{n, k} X^{k}=f_{\mathcal{S}_{n}}=t_{\mathcal{S}_{n}}(X-1)=$ $\sum_{j=0}^{n} \frac{1}{j !}(X-1)^{j}=\sum_{j=0}^{n} \sum_{k=0}^{j} \frac{1}{j !} \cdot(-1)^{j-k}\left(\begin{array}{l}j \\ k\end{array}\right) X^{k}=\sum_{k=0}^{n} \sum_{j=k}^{n} \frac{(-1)^{j-k}}{k !(j-k) !} X^{k}=$ $\sum_{k=0}^{n}\left(\sum_{j=0}^{n-k} \frac{(-1)^{j}}{j !}\right) \frac{X^{k}}{k !} \in \mathbb{Q}[X]$. From this we recover the formula $D_{n}=D_{n, 0}=$ $n ! \cdot \sum_{j=0}^{n} \frac{(-1)^{j}}{j !}$ for derangement numbers, as well as $D_{n, k}=\frac{n !}{k !} \cdot \sum_{j=0}^{n-k} \frac{(-1)^{j}}{j !}=$ $\frac{n !}{k !} \cdot \frac{D_{n-k}}{(n-k) !}=\left(\begin{array}{l}n \\ k\end{array}\right) \cdot D_{n-k}$. Moreover, for $n \rightarrow \infty$ the proportion of elements having precisely $k$ fixed points amongst all permutations is given as $\frac{D_{n, k}}{n !} \rightarrow \frac{1}{e \cdot k !}$, that is a Poisson distribution with parameter 1 .

(15.9) Example: The structure of benzene. The aromatic hydrocarbon compound benzene, which was discovered around 1825, and is nowadays known to have the chemical sum formula $C_{6} H_{6}$, consists of 6 carbon atoms and 6 hydrogen atoms. Due to its chemical stability it was conjectured that the carbon atoms form the vertices of a highly symmetric polyhedron, in which the vertices cannot be distinguished from each other; in particular any vertex is adjacent to the same number of vertices, all edges have the same length, and at any vertex the same types of faces meet. There are three geometrically sensible possible configurations: i) the regular 6-gon, ii) the rectangular prism over an equilateral triangle, and iii) the regular octahedron; see Table 14.

We try to distinguish these cases by producing compounds where two of the hydrogen atoms are replaced by other, distinct compounds, yielding molecules having chemical sum formula $C_{6} H_{4} X Y$, where $X \neq Y$. We ask ourselves how many distinct compounds can be formed like this, assuming either of the above three configurations, where compounds are the same if they can be transformed into each other by rotations.

Thus we have to consider the group of symmetries induced by the special orthogonal group $\mathrm{SO}_{3}(\mathbb{R})$. Its action on the vertices $N:=\{1, \ldots, 6\}$ yields transitive subgroups $G^{(i)} \leq \mathcal{S}_{6}$, for $i \in\{1, \ldots, 3\}$. Hence the new compounds are given as the orbits of $G^{(i)}$ on the set $(N \times N) \backslash\{[j, j] \in N \times N ; j \in N\}$ of pairs of distinct vertices, where the Cauchy-Frobenius-Burnside Lemma yields 
$\left|G^{(i)} \backslash(N \times N)\right|=\frac{1}{\left|G^{(i)}\right|} \cdot \sum_{\pi \in G^{(i)}}\left|\operatorname{Fix}_{N}(\pi)\right|^{2}$.

To determine $G^{(i)}$ we proceed as follows: We have $\left[G^{(i)}: G_{1}^{(i)}\right]=6$, hence we first find $G_{1}^{(i)}$, and then find further elements of $G^{(i)}$ until we generate a transitive subgroup of $\mathcal{S}_{6}$, then the group generated is contained in $G^{(i)}$ and contains $G_{1}^{(i)}$ of index at least 6 , hence coincides with $G^{(i)}$. Having found $G^{(i)}$ explicitly, we determine $\left|\operatorname{Fix}_{N}(\pi)\right|$ for all $\pi \in G^{(i)}$ :

i) For the regular 6 -gon we have the dihedral group $G^{(1)}=D_{12}$ of order 12 , see (15.3). Hence $G_{1}^{(1)}=\left\langle\sigma_{6}\right\rangle=\langle(2,6)(3,5)\rangle$ has order 2, and thus $G^{(1)}=$ $\left\langle\sigma_{6}, \tau_{6}\right\rangle=\langle(2,6)(3,5),(1,2,3,4,5,6)\rangle$. Moreover, the only non-identity elements having fixed points are the three reflections with axis running through a pair of opposite vertices, the latter being the respective fixed points. Hence we find $\left|G^{(1)} \backslash(N \times N)\right|=\frac{1}{12} \cdot\left(6^{2}+3 \cdot 2^{2}\right)=4$, leading to three distinct compounds; in terms of the positions at which $X$ and $Y$ are located, since rotations are distance-preserving, orbit representatives are given by $\{[1,2],[1,3],[1,4]\}$.

ii) For the prism we have $G_{1}^{(2)}=\{1\}$, hence $\left|G^{(2)}\right|=6$, and thus $G^{(2)}=$ $\langle(1,2,3)(4,5,6),(1,4)(2,6)(3,5)\rangle$. Moreover, since $G_{j}^{(2)}=\{1\}$ for all $j \in N$ we conclude that no non-identity element has a fixed point, implying $\mid G^{(2)} \backslash(N \times$ $N) \mid=\frac{6^{2}}{6}=6$, leading to five distinct compounds; similar to the above argument we find that orbit representatives are given by $\{[1,2], \ldots,[1,6]\}$.

iii) For the regular octahedron we have $G_{1}^{(3)}=\langle(2,3,4,5)\rangle$, having order 4 , hence $\left|G^{(3)}\right|=24$, and thus $G^{(3)}:=\langle(2,3,4,5),(1,3,6,5)\rangle$. Moreover, we have $G_{1}^{(3)}=G_{6}^{(3)}$ and $G_{2}^{(3)}=G_{4}^{(3)}$ and $G_{3}^{(3)}=G_{5}^{(3)}$, where any of the non-identity elements of the various stabilizers has precisely two fixed points, showing that $\left|G^{(2)} \backslash(N \times N)\right|=\frac{1}{24} \cdot\left(6^{2}+3 \cdot 3 \cdot 2^{2}\right)=3$, leading to two distinct compounds; similarly we find that orbit representatives are given by $\{[1,2],[1,6]\}$.

In practice, it is found that there are three distinct compounds arising this way, thus giving a strong indication that case (i) is correct. Nowadays it is commonly believed that case (i), the KEKULÉ structure [1865], describes benzene correctly. Case (ii) was suggested by LADENBURG as the structure of benzene, and has been synthesized as Prisman as late as in 1973. Case (iii) does not make sense chemically; nowadays there are various other compounds with chemical sum formula $\mathrm{C}_{6} \mathrm{H}_{6}$ known which possess less symmetries.

\section{Action on maps}

(16.1) Maps. a) Let $N:=\{1, \ldots, n\}$ and $K:=\{1, \ldots, k\}$, for some $n \in$ $\mathbb{N}_{0}$ and $k \in \mathbb{N}_{0}$. The symmetric group $\mathcal{S}_{k}$ acts on the set $\operatorname{Maps}(N, K)$ by post-multiplication, that is $f \mapsto \sigma(f): i \mapsto \sigma(f(i))$, for all $\sigma \in \mathcal{S}_{k}$ : For $\tau \in \mathcal{S}_{k}$ we have $(\sigma \tau)(f)=\sigma(\tau(f)): i \mapsto(\sigma \tau)(f(i))=\sigma(\tau(f(i)))$. Writing $f=[f(1), \ldots, f(n)]$, then $\sigma \in \mathcal{S}_{k}$ acts by renaming the entries.

The symmetric group $\mathcal{S}_{n}$ acts on $\operatorname{Maps}(N, K)$ by pre-multiplication, that 
Table 14: Possible structures of benzene.
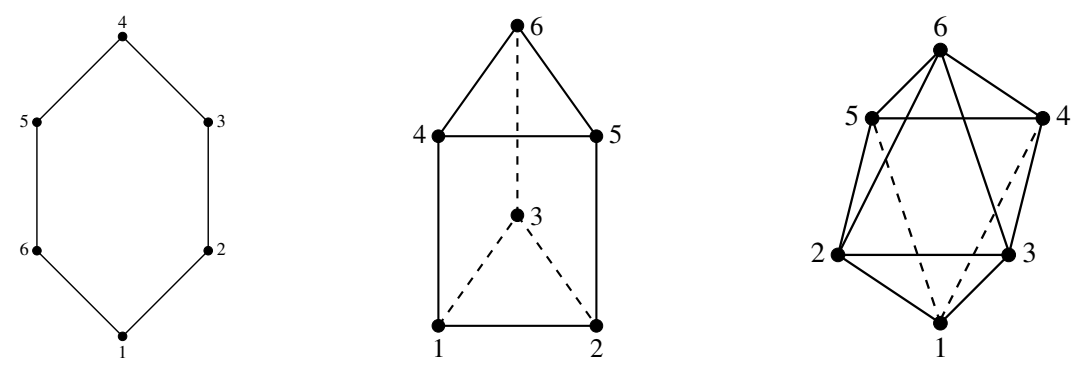

is $f \mapsto{ }^{\pi} f: i \mapsto f\left(\pi^{-1}(i)\right)$, for all $\pi \in \mathcal{S}_{n}$ : For $\mu \in \mathcal{S}_{n}$ we have $\left.{ }^{\pi \mu} f\right)(i)=$ $f\left((\pi \mu)^{-1}(i)\right)=f\left(\left(\mu^{-1} \pi^{-1}\right)(i)\right)=f\left(\mu^{-1}\left(\pi^{-1}(i)\right)\right)=\left({ }^{\mu} f\right)\left(\pi^{-1}(i)\right)=\left({ }^{\pi}(\mu f)\right)(i)$, for all $i \in N$, that is ${ }^{\pi \mu} f=\pi\left({ }^{\mu} f\right)$. Writing $f=[f(1), \ldots, f(n)]$, then $\pi \in \mathcal{S}_{n}$ acts by reordering the entries.

The Cartesian product $\mathcal{S}_{n} \times \mathcal{S}_{k}$ becomes a group with componentwise multiplication and inversion, having neutral element [1,1]. Hence $\mathcal{S}_{n}$ and $\mathcal{S}_{k}$ can be considered as subgroups of $\mathcal{S}_{n} \times \mathcal{S}_{k}$ via the embeddings $\mathcal{S}_{n} \rightarrow \mathcal{S}_{n} \times \mathcal{S}_{k}: \pi \mapsto[\pi, 1]$ and $\mathcal{S}_{k} \rightarrow \mathcal{S}_{n} \times \mathcal{S}_{k}: \sigma \mapsto[1, \sigma]$, respectively, and as such we have $\pi \sigma=\sigma \pi$ for all $\pi \in \mathcal{S}_{n}$ and $\sigma \in \mathcal{S}_{k}$. Moreover we have ${ }^{\pi}(\sigma(f))=\sigma\left({ }^{\pi} f\right): i \mapsto \sigma\left(f\left(\pi^{-1}(i)\right)\right)$, for all $f \in \operatorname{Maps}(N, K)$, thus defining an action of $\mathcal{S}_{n} \times \mathcal{S}_{k}$ on $\operatorname{Maps}(N, K)$.

Hence we may consider the orbits of either of the groups $\{1\} \times\{1\}$ and $\mathcal{S}_{n} \times\{1\}$ as well as $\{1\} \times \mathcal{S}_{k}$ and $\mathcal{S}_{n} \times \mathcal{S}_{k}$ on $\operatorname{Maps}(N, K)$. The elements of $N$ are called indistinguishable if we let $\mathcal{S}_{n}$ act, otherwise they are called distinguishable, and similarly for $K$ and the action of $\mathcal{S}_{k}$. Maps being in the same orbit with respect to either of these groups are called equivalent; if the elements of both $N$ and $K$ are distinguishable, then all equivalence classes are singleton sets.

b) For example, for $n=3$ and $k=4$ let $a, b, c, d \in \operatorname{Maps}(N, K)$ be the pairwise distinct maps $a:=[1,1,2]$ and $b:=[1,2,1]$ and $c:=[2,2,4]$ and $d:=[3,2,2]$; see Table 15. Equivalence with respect to $\mathcal{S}_{n}$, amounting to arbitrary reordering, is given by $\{\{a, b\},\{c\},\{d\}\}$; equivalence with respect to $\mathcal{S}_{k}$, amounting to arbitrary renaming, is given by $\{\{a, c\},\{b\},\{d\}\}$; with respect to $\mathcal{S}_{n} \times \mathcal{S}_{k}$, that is up to arbitrary reordering and renaming, $\{a, b, c, d\}$ are pairwise equivalent.

More intuitively, we may think of $N$ as numbered balls, of $K$ as numbered boxes, and of $f \in \operatorname{Maps}(N, K)$ as a recipe to put the balls into the boxes. This describes the case where both $N$ and $K$ are distinguishable, while if we ignore the numbers on the balls then $N$ becomes indistinguishable, and if we ignore the numbers on the boxes then $K$ becomes indistinguishable. 
Table 15: Balls in boxes.

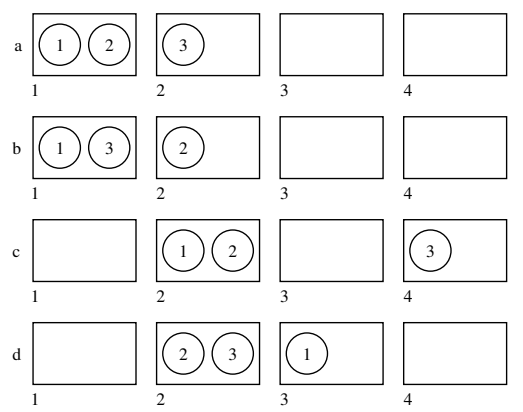

(16.2) The 16-fold way. We continue to consider the action of $\mathcal{S}_{n} \times \mathcal{S}_{k}$ on $\operatorname{Maps}(N, K)$, where $N:=\{1, \ldots, n\}$ and $K:=\{1, \ldots, k\}$, for some $n \in \mathbb{N}_{0}$ and $k \in \mathbb{N}_{0}$. Then a map $f \in \operatorname{Maps}(N, K)$ is injective if and only if $\sigma\left(^{\pi} f\right)$ is, and similarly $f$ is surjective if and only if $\sigma\left({ }^{\pi} f\right)$ is, for all $\pi \in \mathcal{S}_{n}$ and $\sigma \in \mathcal{S}_{k}$. Hence $\operatorname{Inj}(N, K) \subseteq \operatorname{Maps}(N, K)$ and $\operatorname{Surj}(N, K) \subseteq \operatorname{Maps}(N, K)$ as well as $\operatorname{Bij}(N, K) \subseteq \operatorname{Maps}(N, K)$ are also acted on by $\mathcal{S}_{n} \times \mathcal{S}_{k}$.

The number of equivalence classes in $\operatorname{Maps}(N, K)$ and the subsets mentioned, with respect to the action of the obvious subgroups of $\mathcal{S}_{n} \times \mathcal{S}_{k}$, are given in Table 16: The entries for $\{1\} \times\{1\}$ have already been determined in (2.1) and (3.1). From this we also get the entries in the column for $|\operatorname{Bij}(N, K)|$.

For $\mathcal{S}_{n} \times\{1\}$ we get: Since the $\mathcal{S}_{n}$-action amounts to arbitrary reordering of the entries of $n$-tuples, the equivalence classes of $\operatorname{Maps}(N, K)$ and of $\operatorname{Inj}(N, K)$ are in bijection with the $n$-multisets $\mathcal{M}_{n}(k)$ and the $n$-subsets $\mathcal{P}_{n}(k)$ of $K$, respectively; see (2.2) and (2.1). If $k \leq n$, then a map $f \in \operatorname{Maps}(N, K)$ is surjective if and only if it represents an $n$-multiset $\mu=\left[\mu_{1}, \ldots, \mu_{k}\right] \in \mathcal{M}_{n}(k)$ of $K$ such that $\mu_{i} \geq 1$ for all $i \in\{1, \ldots, k\}$, where the latter are in bijection with $\mathcal{M}_{n-k}(k)$ via $\mu \mapsto\left[\mu_{1}-1, \ldots, \mu_{k}-1\right]$.

For $\{1\} \times \mathcal{S}_{k}$ we get: Since the $\mathcal{S}_{k}$-action amounts to arbitrary renaming of the entries of tuples over $K$, the equivalence classes of $\operatorname{Maps}(N, K)$ and of $\operatorname{Surj}(N, K)$ are in bijection, via $f \mapsto \coprod_{i=1}^{k} f^{-1}(i)$, with the partitions of $N$ into at most $k$ blocks and into precisely $k$ blocks, respectively; see (3.1). Moreover, if $n \leq k$, then $\operatorname{Inj}(N, K)$ consists of a single equivalence class.

For $\mathcal{S}_{n} \times \mathcal{S}_{k}$ we get: Since the $\left(\mathcal{S}_{n} \times \mathcal{S}_{k}\right)$-action amounts to arbitrary reordering and renaming of tuples in $K^{n}$, the equivalence classes of $\operatorname{Maps}(N, K)$ and of $\operatorname{Surj}(N, K)$ are in bijection, via $f \mapsto\left[\left|f^{-1}(1)\right|, \ldots,\left|f^{-1}(k)\right|\right]$, with the sets $P_{\leq k}(n)$ and $P_{k}(n)$ of partitions of $n$ into at most $k$ parts and into precisely $k$ parts, respectively; see (3.2). Moreover, if $n \leq k$, then $\operatorname{Inj}(N, K)$ still consists of a single equivalence class. 
Table 16: The 16-fold way.

\begin{tabular}{|c||c|c|c|c|}
\hline & $|\operatorname{Maps}(N, K)|$ & $|\operatorname{Inj}(N, K)|$ & $|\operatorname{Surj}(N, K)|$ & $|\operatorname{Bij}(N, K)|$ \\
\hline \hline$\{1\} \times\{1\}$ & $k^{n}$ & $k_{(n)}$ & $k ! \cdot S_{n, k}$ & $\begin{array}{r}n !, \text { if } n=k \\
0, \text { if } n \neq k\end{array}$ \\
\hline $\mathcal{S}_{n} \times\{1\}$ & $\left(\begin{array}{c}n+k-1 \\
n\end{array}\right)$ & $\left(\begin{array}{l}k \\
n\end{array}\right)$ & $\begin{array}{r}\left(\begin{array}{c}n-1 \\
n-k\end{array}\right), \text { if } n \geq k \\
0, \text { if } n<k\end{array}$ & $\begin{array}{c}1, \text { if } n=k \\
0, \text { if } n \neq k\end{array}$ \\
\hline$\{1\} \times \mathcal{S}_{k}$ & $\sum_{l=0}^{k} S_{n, l}$ & $\begin{array}{c}1, \text { if } n \leq k \\
0, \text { if } n>k\end{array}$ & $S_{n, k}$ & $\begin{array}{c}1, \text { if } n=k \\
0, \text { if } n \neq k\end{array}$ \\
\hline $\mathcal{S}_{n} \times \mathcal{S}_{k}$ & $p_{n, \leq k}$ & $\begin{array}{c}1, \text { if } n \leq k \\
0, \text { if } n>k\end{array}$ & $p_{n, k}$ & $\begin{array}{c}1, \text { if } n=k \\
0, \text { if } n \neq k\end{array}$ \\
\hline
\end{tabular}

(16.3) Example: Shuffles. We consider shuffles of a deck of $n \in \mathbb{N}_{0}$ of cards. Usually a deck of cards is viewed as the sequence of cards appearing while running through it, from top to bottom say. Thus we let a deck be a bijection $\delta: N \rightarrow K$ from the set of positions $N:=\{1, \ldots, n\}$ to a set $K$ of cardinality $n$, hence can be identified with the tuple $[\delta(1), \ldots, \delta(n)]$.

a) We consider the perfect Riffle shuffles: For $n \in \mathbb{N}$ even, divide the deck into its top and bottom halves of the same size, and then interleave the halves perfectly. Then the top card of either the top or the bottom half ends up at the top of the resulting deck, being called the out-shuffle and the in-shuffle, respectively. In terms of the positions $N$ this yields permutations $\omega_{n} \in \mathcal{S}_{n}$ and $\iota_{n} \in \mathcal{S}_{n}$, respectively, writing maps from top to bottom, where $m \in \mathbb{N}$ :

$$
\begin{aligned}
\omega_{2 m} & =\left[\begin{array}{cccccccccc}
1 & m+1 & 2 & m+2 & \ldots & m-1 & 2 m-1 & m & 2 m \\
1 & 2 & 3 & 4 & \ldots & 2 m-3 & 2 m-2 & 2 m-1 & 2 m
\end{array}\right] \\
\iota_{2 m} & =\left[\begin{array}{cccccccccc}
m+1 & 1 & m+2 & 2 & \ldots & 2 m-1 & m-1 & 2 m & m \\
1 & 2 & 3 & 4 & \ldots & 2 m-3 & 2 m-2 & 2 m-1 & 2 m
\end{array}\right] \\
\omega_{2 m-1} & =\left[\begin{array}{cccccccccc}
1 & m+1 & 2 & m+2 & \ldots & 2 m-2 & m-1 & 2 m-1 & m \\
1 & 2 & 3 & 4 & \ldots & 2 m-4 & 2 m-3 & 2 m-2 & 2 m-1
\end{array}\right] \\
\iota_{2 m-1} & =\left[\begin{array}{ccccccccc}
m & 1 & m+1 & 2 & \ldots & m-2 & 2 m-2 & m-1 & 2 m-1 \\
1 & 2 & 3 & 4 & \ldots & 2 m-4 & 2 m-3 & 2 m-2 & 2 m-1
\end{array}\right]
\end{aligned}
$$

For example, for $n=8$ we get:

$$
\begin{aligned}
\omega_{8} & =\left[\begin{array}{llllllll}
1 & 5 & 2 & 6 & 3 & 7 & 4 & 8 \\
1 & 2 & 3 & 4 & 5 & 6 & 7 & 8
\end{array}\right]=(2,3,5)(4,7,6) \\
\iota_{8} & =\left[\begin{array}{llllllll}
5 & 1 & 6 & 2 & 7 & 3 & 8 & 4 \\
1 & 2 & 3 & 4 & 5 & 6 & 7 & 8
\end{array}\right]=(1,2,4,8,7,5)(3,6)
\end{aligned}
$$

Iterating the shuffling corresponds to multiplying the associated permutations: 
For example, for $n=8$ performing an in-shuffle followed by an out-shuffle yields $\omega_{8} \iota_{8}=(2,3,5)(4,7,6) \cdot(1,2,4,8,7,5)(3,6)=(1,3,4,8,6,5)(2,7)$, while the other way around we get $\iota_{8} \omega_{8}=(1,2,4,8,7,5)(3,6) \cdot(2,3,5)(4,7,6)=$ $(1,2,6,8,7,3)(4,5)$. This translates back into decks of cards as follows:

$$
\begin{aligned}
& \omega_{8} \iota_{8}=(1,3,4,8,6,5)(2,7)=\left[\begin{array}{llllllll}
5 & 7 & 1 & 3 & 6 & 8 & 2 & 4 \\
1 & 2 & 3 & 4 & 5 & 6 & 7 & 8
\end{array}\right] \\
& \iota_{8} \omega_{8}=(1,2,6,8,7,3)(4,5)=\left[\begin{array}{llllllll}
3 & 1 & 7 & 5 & 4 & 2 & 8 & 6 \\
1 & 2 & 3 & 4 & 5 & 6 & 7 & 8
\end{array}\right]
\end{aligned}
$$

Considering the Riffle shuffle group $\mathcal{R}_{n}:=\left\langle\omega_{n}, \iota_{n}\right\rangle \leq \mathcal{S}_{n}$, for $n \geq 2$, we experimentally find the following pattern: $\mathcal{R}_{n}$ is transitive; $\mathcal{R}_{n}$ is tiny compared to $\mathcal{S}_{n}$ whenever $n$ is odd, of order at most $n(n-1) ; \mathcal{R}_{n}$ is small compared to $\mathcal{S}_{n}$ whenever $n$ is even, where for $m \notin\{6,12\} \dot{\cup}\left\{2^{k} ; k \geq 0\right\}$ we get $\left|\mathcal{R}_{2 m}\right|=2^{m-1} \cdot \frac{m !}{2}$ whenever $m \equiv 0(\bmod 4)$, and $\left|\mathcal{R}_{2 m}\right|=2^{m} \cdot \frac{m !}{2}$ whenever $m \equiv 1(\bmod 4)$, and $\left|\mathcal{R}_{2 m}\right|=2^{m} \cdot m$ ! whenever $m \equiv 2(\bmod 4)$, and $\left|\mathcal{R}_{2 m}\right|=2^{m-1} \cdot m$ ! whenever $m \equiv 3(\bmod 4)$, while for $k \geq 1$ we find $\left|\mathcal{R}_{2^{k}}\right|=2^{k} \cdot k$, and $\left|\mathcal{R}_{12}\right|=2^{6} \cdot 120$ and $\left|\mathcal{R}_{24}\right|=2^{11} \cdot 95040$; note that this indicates a close relationship between $\mathcal{R}_{2 m}$ and the Mongean shuffle groups $\mathcal{M}_{m}$ discussed now:

b) The Mongean shuffles are given as follows: Start with the topmost card, and then put every other card on the top and on the bottom, respectively on the bottom and on the top. Then if $n \in \mathbb{N}$ is even the last card ends up at the top and the bottom, respectively, and the other way around if $n \in \mathbb{N}$ is odd. In terms of the positions $N$ this yields permutations $\mu_{n} \in \mathcal{S}_{n}$ and $\mu_{n}^{\prime} \in \mathcal{S}_{n}$, respectively, where $m \in \mathbb{N}$ :

$$
\begin{aligned}
\mu_{2 m} & =\left[\begin{array}{cccccccccc}
2 m & 2 m-2 & \ldots & 4 & 2 & 1 & 3 & \ldots & 2 m-1 \\
1 & 2 & \ldots & m-1 & m & m+1 & m+2 & \ldots & 2 m
\end{array}\right] \\
\mu_{2 m}^{\prime} & =\left[\begin{array}{ccccccccc}
2 m-1 & 2 m-3 & \ldots & 3 & 1 & 2 & 4 & \ldots & 2 m \\
1 & 2 & \ldots & m-1 & m & m+1 & m+2 & \ldots & 2 m
\end{array}\right] \\
\mu_{2 m-1} & =\left[\begin{array}{ccccccccc}
2 m-2 & 2 m-4 & \ldots & 2 & 1 & 3 & \ldots & 2 m-1 \\
1 & 2 & \ldots & m-1 & m & m+1 & \ldots & 2 m-1
\end{array}\right] \\
\mu_{2 m-1}^{\prime} & =\left[\begin{array}{cccccccc}
2 m-1 & 2 m-3 & \ldots & 3 & 1 & 2 & \ldots & 2 m-2 \\
1 & 2 & \ldots & m-1 & m & m+1 & \ldots & 2 m-1
\end{array}\right]
\end{aligned}
$$

For example, for $n=8$ we get:

$$
\begin{aligned}
& \mu_{8}=\left[\begin{array}{llllllll}
8 & 6 & 4 & 2 & 1 & 3 & 5 & 7 \\
1 & 2 & 3 & 4 & 5 & 6 & 7 & 8
\end{array}\right]=(1,5,7,8)(2,4,3,6) \\
& \mu_{8}^{\prime}=\left[\begin{array}{llllllll}
7 & 5 & 3 & 1 & 2 & 4 & 6 & 8 \\
1 & 2 & 3 & 4 & 5 & 6 & 7 & 8
\end{array}\right]=(1,4,6,7)(2,5)
\end{aligned}
$$

Considering the Mongean shuffle group $\mathcal{M}_{n}:=\left\langle\mu_{n}, \mu_{n}^{\prime}\right\rangle \leq \mathcal{S}_{n}$, for $n \geq 2$, we experimentally find the following pattern: If $n \notin\{6,12\} \dot{\cup}\left\{2^{k} ; k \geq 3\right\}$, then 
$\mathcal{M}_{n}=\mathcal{S}_{n}$ whenever $n \equiv\{2,3\}(\bmod 4)$, and $\mathcal{M}_{n}=\mathcal{A}_{n}$ whenever $n \equiv\{0,1\}$ $(\bmod 4)$, while for $k \geq 3$ we find $\left|\mathcal{M}_{2^{k}}\right|=2^{k} \cdot(k+1)$, and we get $\left|\mathcal{M}_{6}\right|=120=\frac{6 !}{6}$ and $\left|\mathcal{M}_{12}\right|=95040=\frac{12 !}{5040}$.

\section{Polya's Theorem}

If a counting problem is translated into a question concerning equivalence classes of maps, where symmetries of the objects considered have to be taken into account, the groups acting by pre- oder post-multiplication might be smaller than the full symmetric groups. We are going to consider the action of a group $G \cong G \times\{1\} \leq \mathcal{S}_{n} \times \mathcal{S}_{k}$ on the full set $\operatorname{Maps}(N, K)$.

(17.1) The cycle index. Let $n \in \mathbb{N}_{0}$, and let $X_{1}, \ldots, X_{n}$ be indeterminates. For $\pi \in \mathcal{S}_{n}$, letting $\lambda(\pi)=\left[n^{a_{n}(\pi)}, \ldots, 1^{a_{1}(\pi)}\right] \in P_{k(\pi)}(n)$ be its cycle type, let $c_{\pi}:=\prod_{i=1}^{n} X_{i}^{a_{i}(\pi)} \in \mathbb{Z}\left[X_{1}, \ldots, X_{n}\right]$ be its cycle monomial. Hence $c_{\pi}$ is monic of degree $\sum_{i=1}^{n} a_{i}(\pi)=k(\pi)$, thus $c_{\pi}(X, \ldots, X)=X^{k(\pi)} \in \mathbb{Z}[X]$; but if $X_{i}$ is given degree $i$, for all $i \in\{1, \ldots, n\}$, then $c_{\pi}$ has weighted degree $n$.

Let $G$ be a finite group acting faithfully on the set $N:=\{1, \ldots, n\}$ via $\varphi: G \rightarrow$ $\mathcal{S}_{n}$, where $n \in \mathbb{N}_{0}$. Then letting $c_{g}:=c_{\varphi(g)} \in \mathbb{Z}\left[X_{1}, \ldots, X_{n}\right]$ be the associated cycle monomials, the polynomial $c_{G}:=\frac{1}{|G|} \cdot \sum_{g \in G} c_{g} \in \mathbb{Q}\left[X_{1}, \ldots, X_{n}\right]$ is called the cycle index of $G$; note that $c_{G}$ only depends on the equivalence class of $G$-actions considered, and that $c_{G}(1, \ldots, 1)=1$. The cycle index has degree $n$, the only monomial of degree $n$ appearing is $X_{1}^{n}$, with coefficient $\frac{1}{|G|}$, and evaluating yields $c_{G}(1, \ldots, 1)=1$; but for the weighted degree we observe that $c_{G}$ is homogeneous of degree $n$. For example, for $\{1\} \leq \mathcal{S}_{n}$ we get $c_{\{1\}}=X_{1}^{n} \in$ $\mathbb{Q}\left[X_{1}, \ldots, X_{n}\right]$.

Hence using the $\mathbb{Q}$-algebra homomorphism $\mathbb{Q}\left[X_{1}, \ldots, X_{n}\right] \rightarrow \mathbb{Q}[X]$ given by $X_{1} \mapsto X$, and $X_{i} \mapsto 1$ for $i \in\{2, \ldots, n\}$, we recover the fixed point index $f_{G}=$ $c_{G}(X, 1, \ldots, 1) \in \mathbb{Q}[X]$. More interestingly, using the $\mathbb{Q}$-algebra homomorphism $\mathbb{Q}\left[X_{1}, \ldots, X_{n}\right] \rightarrow \mathbb{Q}[X]: X_{i} \mapsto X$, for all $i \in\{1, \ldots, n\}$, the cycle-number index is given as $k_{G}=k_{G, N}:=c_{G}(X, \ldots, X)=\frac{1}{|G|} \cdot \sum_{g \in G} X^{k(g)} \in \mathbb{Q}[X]$, where $k(g):=k(\varphi(g)) \in \mathbb{N}_{0}$ is the number of cycles of $g$.

We show that the cycle index indeed depends on the $G$-action considered, not just only on the isomorphism class of $G$ : By way of example, let $G:=$ $\langle(1,2)(3,4),(1,4)(2,3)\rangle=\{(),(1,2)(3,4),(1,3)(2,4),(1,4)(2,3)\}$ as well as $H:=$ $\langle(1,3),(2,4)\rangle=\{(),(1,3),(2,4),(1,3)(2,4)\}$; actually we have $G, H \leq D_{8} \leq \mathcal{S}_{4}$, see Table 13. Then $G \cong H$ as groups via $(1,2)(3,4) \mapsto(1,3)$ and $(1,4)(2,3) \mapsto$ $(2,4)$, hence $(1,3)(2,4) \mapsto(1,3)(2,4)$, but we have $c_{G}=\frac{1}{4}\left(X_{1}^{4}+3 X_{2}^{2}\right) \neq$ $\frac{1}{4}\left(X_{1}^{4}+2 X_{2} X_{1}^{2}+X_{2}^{2}\right)=c_{H} \in \mathbb{Q}\left[X_{1}, \ldots, X_{4}\right]$.

Moreover, groups having one and the same cycle index are not necessarily equivalent as permutation groups, and actually are not necessarily isomorphic: By way of example, let $p$ be an odd prime, let $G$ be the elementary-abelian group 
of order $p^{3}$, and let $H$ be the extra-special group of order $p^{3}$ of exponent $p$, then with respect to their regular representations both groups have cycle index $c_{G}=c_{H}=\frac{1}{p^{3}}\left(X_{1}^{p^{3}}+\left(p^{3}-1\right) X_{p}^{3}\right) \in \mathbb{Q}\left[X_{1}, \ldots, X_{p^{3}}\right]$.

(17.2) Example: Cycle index of $\mathcal{S}_{n}$. For $n \in \mathbb{N}_{0}$ and a partition $\lambda=$ $\left[n^{a_{n}}, \ldots, 1^{a_{1}}\right] \vdash n$ there are $\frac{n !}{\prod_{i=1}^{n}\left(a_{i} ! \cdot i^{a_{i}}\right)}$ permutations in $\mathcal{S}_{n}$ with cycle type $\lambda$, implying $c_{n}:=c_{\mathcal{S}_{n}}=\sum_{\lambda=\left[n^{a_{n}}, \ldots, 1^{a_{1}}\right] \vdash n}\left(\prod_{i=1}^{n} \frac{1}{a_{i} ! \cdot i^{a_{i}}} X_{i}^{a_{i}}\right) \in \mathbb{Q}\left[X_{1}, \ldots, X_{n}\right]$. We aim at considering the cycle indices $c_{n}$ for all $n \in \mathbb{N}_{0}$ simultaneously:

To this end, let $\mathbb{Q}[\mathcal{X}]$ be the polynomial ring in countably infinitely many indeterminates $\mathcal{X}:=\left\{X_{i} ; i \in \mathbb{N}\right\}$; hence we have $\mathbb{Q} \subseteq \mathbb{Q}\left[X_{1}\right] \subseteq \mathbb{Q}\left[X_{1}, X_{2}\right] \subseteq$ $\cdots \subseteq \mathbb{Q}[\mathcal{X}]$. Let $c:=\sum_{n \geq 0} c_{n} X^{n} \in \mathbb{Q}[\mathcal{X}][[X]]$ be the generating series associated to the sequence $\left[c_{n} ; n \in \mathbb{N}_{0}\right] \in \operatorname{Maps}\left(\mathbb{N}_{0}, \mathbb{Q}[\mathcal{X}]\right)$. Hence we have $c=\sum_{n \geq 0}\left(\sum_{\lambda=\left[n^{a_{n}}, \ldots, 1^{a_{1}}\right] \vdash n} \prod_{i=1}^{n} \frac{1}{a_{i} ! \cdot^{a_{i}}} X_{i}^{a_{i}}\right) X^{n} \in \mathbb{Q}[\mathcal{X}][[X]]$.

Identifying the set $\coprod_{n>0} P(n)$ of all partitions with the set $\mathcal{M}:=\left\{\left[a_{1}, a_{2}, \ldots\right] \in\right.$ $\operatorname{Maps}\left(\mathbb{N}, \mathbb{N}_{0}\right) ; a_{i}=0$ for almost all $\left.i \in \mathbb{N}\right\}$, where hence $a=\left[a_{1}, a_{2}, \ldots\right] \in$ $\mathcal{M}$ represents a partition of $n=\sum_{i \geq 1} i a_{i}$, see (14.1), the generating series becomes $c=\sum_{a \in \mathcal{M}}\left(\prod_{i \geq 1} \frac{1}{a_{i} ! \cdot i^{a_{i}}} X_{i}^{a_{i}} X^{i a_{i}}\right)=\prod_{i \geq 1}\left(\sum_{a_{i} \geq 0} \frac{1}{a_{i} !}\left(\frac{1}{i} X_{i} X^{i}\right)^{a_{i}}\right)=$ $\prod_{i \geq 1} \exp \left(\frac{1}{i} X_{i} X^{i}\right)=\exp \left(\sum_{i \geq 1} \frac{1}{i} X_{i} X^{i}\right) \in \mathbb{Q}[\mathcal{X}][[X]]$.

(17.3) Cycle index of Galois groups. a) Let $f=X^{n}+\sum_{i=1}^{n} a_{n-i} X^{n-i} \in$ $\mathbb{Z}[X]$ be an irreducible monic polynomial of degree $n=\operatorname{deg}(f) \geq 2$, with zeroes $\left\{x_{1}, \ldots, x_{n}\right\} \subseteq \mathbb{C}$, and let $K=\mathbb{Q}\left[x_{1}, \ldots, x_{n}\right] \subseteq \mathbb{C}$ be its splitting field with Galois group $G:=\operatorname{Gal}(K / \mathbb{Q})$; then $G$ acts faithfully and transitively on $\left\{x_{1}, \ldots, x_{n}\right\}$, hence we may view $G$ as a subgroup of $\mathcal{S}_{n}$. Let $\operatorname{disc}(f):=$ $\prod_{1 \leq i<j \leq n}\left(x_{i}-x_{j}\right)^{2} \in \mathbb{Z} \backslash\{0\}$ denote the discriminant of $f$. Then, for any prime $p \nmid \operatorname{disc}(f)$ the reduced polynomial $f_{p} \in \mathbb{F}_{p}[X]$ is separable of degree $n$, and factors as $f_{p}=\prod_{i=1}^{l} f_{p, i} \in \mathbb{F}_{p}[X]$, where $l=l(p) \in \mathbb{N}$ and the $f_{p, i} \in \mathbb{F}_{p}[X]$ are pairwise distinct irreducible monic polynomials, of degree $d_{p, i} \in \mathbb{N}$ say; assuming the $d_{p, i}$ to be suitably ordered, we get $\lambda(p):=\left[d_{p, 1}, \ldots, d_{p, l}\right] \vdash n$.

For any prime $p \nmid \operatorname{disc}(f)$, choose a discrete valuation ring $R_{p} \subseteq K$ with (finite) residue field $\mathbb{F}_{q}$ of characteristic $p$. Then $\mathbb{F}_{q} \subseteq \overline{\mathbb{F}}_{p}$ is the splitting field of $f_{p}$, hence we have $\left[\mathbb{F}_{q}: \mathbb{F}_{p}\right]=\log _{p}(q)=d_{p}=\operatorname{lcm}\left(d_{p, 1}, \ldots, d_{p, l}\right) \in \mathbb{N}$, and thus $\operatorname{Gal}\left(\mathbb{F}_{q} / \mathbb{F}_{p}\right)=\left\langle\varphi_{p}\right\rangle \cong C_{d_{p}}$, where $\varphi_{p}: \mathbb{F}_{q} \rightarrow \mathbb{F}_{q}: x \mapsto x^{p}$ is the associated Frobenius automorphism. Then, since $p \nmid \operatorname{disc}(K / \mathbb{Q}) \mid \operatorname{disc}(f)$ implies that $K / \mathbb{Q}$ is unramified at the prime $p$, we conclude that $\varphi_{p}$ lifts to a unique element $\sigma_{p} \in G$. Taking the choice made into account this determines a unique conjugacy class $\mathcal{C}_{p} \subseteq G$; viewing $G \leq \mathcal{S}_{n}$ the latter consists of elements of cycle type $\lambda(p)$. Now, by Chebotarev's Density Theorem, for any conjugacy class $\mathcal{C} \subseteq G$ the set of primes such that $\mathcal{C}_{p}=C$ has density $\lim _{x \rightarrow \infty} \frac{\mid\left\{p \text { prime; } p \leq x, \mathcal{C}_{p}=C\right\} \mid}{\mid\{p \text { prime; } p \leq x\} \mid}=\frac{|C|}{|G|}$; note that this in particular says that the limit actually exists.

b) Now, the original question behind the Lenstra inequality, see (15.6), was (in the context of the asymptotically fast Number Field Sieve algorithm 
Table 17: Cycle types for $f_{1}$ and $f_{2}$.

\begin{tabular}{|l|l|}
\hline$\lambda$ & proportion \\
\hline \hline$\left[1^{11}\right]$ & $\sim 0.0000$ \\
{$\left[2^{4}, 1^{3}\right]$} & $\sim 0.0538$ \\
{$\left[3^{3}, 1^{2}\right]$} & $\sim 0.1616$ \\
{$\left[5^{2}, 1\right]$} & $\sim 0.4431$ \\
{$[6,3,2]$} & $\sim 0.1616$ \\
{$[11]$} & $\sim 0.1796$ \\
\hline
\end{tabular}

\begin{tabular}{|l|l|}
\hline$\lambda$ & proportion \\
\hline \hline$\left[1^{11}\right]$ & $\sim 0.0000$ \\
{$\left[2^{4}, 1^{3}\right]$} & $\sim 0.006$ \\
{$\left[3^{3}, 1^{2}\right]$} & $\sim 0.0363$ \\
{$\left[4^{2}, 1^{3}\right]$} & $\sim 0.0969$ \\
{$\left[5^{2}, 1\right]$} & $\sim 0.1878$ \\
{$[6,3,2]$} & $\sim 0.1878$ \\
{$[8,2,1]$} & $\sim 0.2848$ \\
{$[11]$} & $\sim 0.2$ \\
\hline
\end{tabular}

for factoring integers), whether there are infinitely many primes $p$ such that $f_{p} \in \mathbb{F}_{p}[X]$ does not have any zeroes in $\mathbb{F}_{p}$, and to give a lower bound for their frequency, independent of the particular Galois group of $f$ :

By the above considerations, $p \nmid \operatorname{disc}(f)$ is as desired if and only if $\mathcal{C}_{p} \subseteq$ $G$ consists of fixed-point-free elements, that is $\mathcal{C}_{p} \subseteq \mathcal{D}$, where we infer that $\lim _{x \rightarrow \infty} \frac{\mid\left\{p \text { prime; } p \leq x, \mathcal{C}_{p} \subseteq \mathcal{D}\right\} \mid}{\mid\{p \text { prime; } p \leq x\} \mid}=\frac{|\mathcal{D}|}{|G|} \geq \frac{1}{n}=\frac{1}{\operatorname{deg}(f)}$.

c) This yields a randomized algorithm to determine the cycle index of $G \leq \mathcal{S}_{n}$ with respect to its transitive action on the (unknown) set $\left\{x_{1}, \ldots, x_{n}\right\}$, and thus to narrow down the conjugacy classes of transitive subgroups of $\mathcal{S}_{n}$ to be considered as possible candidates for $G$. We explain this by an example:

We consider the case $n=11$ and the (sensibly chosen) polynomials

$$
\begin{aligned}
f_{1}= & X^{11}-2 X^{10}+3 X^{9}+2 X^{8}-5 X^{7}+16 X^{6} \\
& -10 X^{5}+10 X^{4}+2 X^{3}-3 X^{2}+4 X-1 \in \mathbb{Z}[X], \\
f_{2}= & X^{11}-3 X^{10}+8 X^{9}-6 X^{8}-9 X^{7}+51 X^{6} \\
& -96 X^{5}+108 X^{4}-66 X^{3}-14 X^{2}+42 X-40 \in \mathbb{Z}[X] ;
\end{aligned}
$$

we have $\operatorname{disc}\left(f_{1}\right)=47^{2} \cdot 1831^{4} \sim 2 \cdot 10^{15}$ and $\operatorname{disc}\left(f_{2}\right)=2^{18} \cdot 3^{18} \cdot 97^{4} \sim 9 \cdot 10^{21}$. Checking the admissible primes not exceeding 1000, that is 167 and 165 primes, respectively, we find the proportion of cycle types as given in Table 17. In particular, since in both cases the cycle type [11] occurs, we conclude that $f_{1}$ and $f_{2}$ actually are irreducible.

Now there are 8 conjugacy classes of transitive subgroups of $\mathcal{S}_{11}$, namely: $C_{11}$, $D_{22}, C_{11}: C_{5}, C_{11}: C_{10}, \mathrm{PSL}_{2}(11), M_{11}, \mathcal{A}_{11}, \mathcal{S}_{11}$. From the cycle types occurring we immediately exclude the first four cases. Moreover, from the discriminants of $f_{1}$ and $f_{2}$ being squares, we conclude the Galois groups are contained in $\mathcal{A}_{11}$, hence this excludes $\mathcal{S}_{11}$ as well. Next, as far as $\mathcal{A}_{11}$ is concerned, the 
Table 18: Cycle types of $L_{2}(11)$ and $M_{11}$.

\begin{tabular}{|l|r|rl|}
\hline$\lambda(g)$ & $\left|g^{G}\right|$ & \multicolumn{2}{|c|}{ proportion } \\
\hline \hline$\left[1^{11}\right]$ & 1 & $\frac{1}{660}$ & $\sim 0.001$ \\
{$\left[2^{4}, 1^{3}\right]$} & 55 & $\frac{1}{12}$ & $\sim 0.083$ \\
{$\left[3^{3}, 1^{2}\right]$} & 110 & $\frac{1}{6}$ & $\sim 0.166$ \\
{$\left[5^{2}, 1\right]$} & 264 & $\frac{2}{5}$ & $\sim 0.4$ \\
{$[6,3,2]$} & 110 & $\frac{1}{6}$ & $\sim 0.166$ \\
{$[11]$} & 120 & $\frac{2}{11}$ & $\sim 0.181$ \\
\hline
\end{tabular}

\begin{tabular}{|l|r|rl|}
\hline$\lambda(g)$ & $\left|g^{G}\right|$ & proportion \\
\hline \hline$\left[1^{11}\right]$ & 1 & $\frac{1}{7920}$ & $\sim 0.0001$ \\
{$\left[2^{4}, 1^{3}\right]$} & 165 & $\frac{1}{48}$ & $\sim 0.0208$ \\
{$\left[3^{3}, 1^{2}\right]$} & 440 & $\frac{1}{18}$ & $\sim 0.0555$ \\
{$\left[4^{2}, 1^{3}\right]$} & 990 & $\frac{1}{8}$ & $\sim 0.125$ \\
{$\left[5^{2}, 1\right]$} & 1584 & $\frac{1}{5}$ & $\sim 0.2$ \\
{$[6,3,2]$} & 1320 & $\frac{1}{6}$ & $\sim 0.1666$ \\
{$[8,2,1]$} & $990+990$ & $\frac{1}{4}$ & $\sim 0.25$ \\
{$[11]$} & $720+720$ & $\frac{2}{11}$ & $\sim 0.1818$ \\
\hline
\end{tabular}

proportion of elements containing a 7 -cycle is $\sim 0.1429$, and of those containing a 9-cycle is $\sim 0.1111$, hence the absence of these elements from the sample taken is a strong indication that the Galois groups looked for are proper subgroups of $\mathcal{A}_{11}$. Hence there are only two groups remaining:

The simple projective special linear group $\mathrm{PSL}_{2}(11)$, acting 2-transitively but not 3-transitively, has order $\left|\mathrm{PSL}_{2}(11)\right|=660=11 \cdot 10 \cdot 6$, and conjugacy classes as given in Table 18, which yields the cycle index $c_{\mathrm{PSL}_{2}(11)}=\frac{1}{660}\left(X_{1}^{11}+\right.$ $55 X_{1}^{3} X_{2}^{4}+110 X_{1}^{2} X_{3}^{3}+264 X_{1} X_{5}^{2}+110 X_{2} X_{3} X_{6}+120 X_{11}$. The sporadic simple Mathieu group $M_{11}$, acting sharply 4-transitively, has order $\left|M_{11}\right|=7920=$ $11 \cdot 10 \cdot 9 \cdot 8$, and conjugacy classes as given in Table 18, which yields the cycle index $c_{M_{11}}=\frac{1}{7920}\left(X_{1}^{11}+165 X_{1}^{3} X_{2}^{4}+990 X_{1}^{3} X_{4}^{2}+440 X_{1}^{2} X_{3}^{3}+1980 X_{1} X_{2} X_{8}+\right.$ $1584 X_{1} X_{5}^{2}+1320 X_{2} X_{3} X_{6}+1440 X_{11}$; note that thus we may also cross-check the fixed point index $f_{M_{11}}$, which was already determined in (15.7).

For $f_{2}$, the existence of elements of cycle type $[8,2,1]$ and $\left[4^{2}, 1^{3}\right]$ excludes the former possibility, so that we have a strong indication that its Galois group is isomorphic to $M_{11}$. Similarly, for $f_{1}$, the absence of elements of the cycle types just mentioned is a strong indication that its Galois group is not isomorphic to $M_{11}$, hence that it is isomorphic to $\mathrm{PSL}_{2}(11)$.

(17.4) The weight index. Let $N:=\{1, \ldots, n\}$ and $K:=\{1, \ldots, k\}$, for some $n \in \mathbb{N}_{0}$ and $k \in \mathbb{N}_{0}$. Let $G$ be a finite group acting faithfully on $N$, with cycle index $c_{G} \in \mathbb{C}\left[X_{1}, \ldots, X_{n}\right]$. The $G$-orbits $G \backslash \operatorname{Maps}(N, K):=\left\{{ }^{G} f \subseteq\right.$ $\operatorname{Maps}(N, K) ; f \in \operatorname{Maps}(N, K)\}$ are called the patterns with respect to the $G$ action, and let $S \subseteq \operatorname{Maps}(N, K)$ be a set of orbit representatives. We aim at determining the number $|G \backslash \operatorname{Maps}(N, K)|$ of patterns:

To this end, let $R:=\mathbb{Q}\left[Y_{1}, \ldots, Y_{k}\right]$, where $Y_{1}, \ldots, Y_{k}$ are indeterminates, being 
called the associated (generic) weight algebra. Let the (generic) weight of $f \in \operatorname{Maps}(N, K)$ be defined as $w(f):=\prod_{i=1}^{n} Y_{f(i)}=\prod_{j=1}^{k} Y_{j}^{\left|f^{-1}(j)\right|} \in R$; hence $w(f)$ is monic of degree $n$. Then for all $\pi \in \mathcal{S}_{n}$ we have $\left.w\left({ }^{\pi} f\right)=\prod_{i=1}^{n} Y_{(\pi} f\right)(i)=$ $\prod_{i=1}^{n} Y_{f\left(\pi^{-1}(i)\right)}=\prod_{i=1}^{n} Y_{f(i)}=w(f)$. Thus weights are constant on $\mathcal{S}_{n}$-orbits, hence the weight $w\left({ }^{G} f\right):=w(f) \in R$ of ${ }^{G} f \in G \backslash \operatorname{Maps}(N, K)$ is well-defined.

Then $\sum_{f \in S} w(f) \in R$ is called the (generic) weight index of $G \backslash \operatorname{Maps}(N, K)$. For $a:=\left[a_{1}, \ldots, a_{k}\right] \in \mathbb{N}_{0}^{k}$ we let $\omega_{a}:=\mid\left\{f \in S ;\left|f^{-1}(j)\right|=a_{j}\right.$ for $j \in$ $\{1, \ldots, k\}\} \mid \in \mathbb{N}_{0}$ be the number of patterns in which $j$ occurs precisely $a_{j}$ times for all $j \in\{1, \ldots, k\}$; note that by the above this is independent from the choice of representatives. Hence we have $\sum_{f \in S} w(f)=\sum_{a=\left[a_{1}, \ldots, a_{k}\right] \in \mathbb{N}_{0}^{k}}\left(\omega_{a}\right.$. $\left.\prod_{j=1}^{k} Y_{j}^{a_{j}}\right) \in R$, that is $\omega_{a}$ is the coefficient of the monomial $\prod_{j=1}^{k} Y_{j}^{a_{j}}$ in the weight index; note that the weight index is homogeneous of degree $n$.

The main result now is the following polynomial identity, relating the weight index of $G \backslash \operatorname{Maps}(N, K)$ to the cycle index of the $G$-action on $N$ :

(17.5) Theorem: Polya [1937]. Keeping the notation of (17.4), we have:

$$
\sum_{f \in S} w(f)=c_{G}\left(\sum_{j=1}^{k} Y_{j}, \sum_{j=1}^{k} Y_{j}^{2}, \ldots, \sum_{j=1}^{k} Y_{j}^{n}\right) \in \mathbb{Q}\left[Y_{1}, \ldots, Y_{k}\right]=: R
$$

using the $\mathbb{Q}$-algebra homomorphism $\mathbb{Q}\left[X_{1}, \ldots, X_{n}\right] \rightarrow R: X_{i} \mapsto \sum_{j=1}^{k} Y_{j}^{i}$.

Proof. Given $f \in S$, then $G$ acts transitively on the $G$-orbit ${ }^{G} f \subseteq \operatorname{Maps}(N, K)$, and the Cauchy-Frobenius-Burnside Lemma yields $1=\frac{1}{|G|} \cdot \sum_{g \in G}\left|\operatorname{Fix}_{\left({ }_{f}\right)}(g)\right|=$ $\frac{1}{|G|} \cdot \sum_{g \in G} \sum_{e \in \operatorname{Fix}(g) \cap(G)} 1 \in \mathbb{Q}$, where we write $\operatorname{Fix}(g):=\operatorname{Fix}_{\operatorname{Maps}(N, K)}(g)$. Since weights are constant on $G$-orbits, multiplication with $w(f) \in R$ yields $w(f)=\frac{1}{|G|} \cdot \sum_{g \in G} \sum_{e \in \operatorname{Fix}(g) \cap\left({ }^{G} f\right)} w(e) \in R$, and thus summation over all $f \in S$ yields the weight index $\sum_{f \in S} w(f)=\frac{1}{|G|} \cdot \sum_{g \in G} \sum_{e \in \operatorname{Fix}(g)} w(e) \in R$.

Given $g \in G$, we consider the inner sum $\sum_{e \in \operatorname{Fix}(g)} w(e) \in R$ : For any map $e \in \operatorname{Maps}(N, K)$ and any $\pi \in \mathcal{S}_{n}$ we have ${ }^{\pi} e=e$ if and only if $e\left(\pi^{-1}(i)\right)=$ $e(i)$ for all $i \in N$, which holds if and only if $e$ is constant on the $k(\pi) \in$ $\mathbb{N}_{0}$ disjoint cycles of $\pi$. Thus we have $e \in \operatorname{Fix}(g)$ if and only if $e$ is constant on the $l:=k(g) \in \mathbb{N}_{0}$ disjoint cycles $N_{1}, \ldots, N_{l} \subseteq N$ of $g$, where the values $e_{1}, \ldots, e_{l} \in K$ of $e$ on the various cycles can be chosen arbitrarily. Thus we get $\sum_{e \in \operatorname{Fix}(g)} w(e)=\sum_{e \in \operatorname{Fix}(g)} \prod_{i=1}^{n} Y_{e_{i}}=\sum_{e \in \operatorname{Fix}(g)} \prod_{s=1}^{l} Y_{e_{s}}^{\left|N_{s}\right|}=$ $\sum_{\left[e_{1}, \ldots, e_{l}\right] \in K^{l}} \prod_{s=1}^{l} Y_{e_{s}}^{\left|N_{s}\right|}=\prod_{s=1}^{l}\left(\sum_{j=1}^{k} Y_{j}^{\left|N_{s}\right|}\right)$.

Hence if $g$ has cycle type $\lambda(g)=\left[n^{a_{n}(g)}, \ldots, 1^{a_{1}(g)}\right] \in P_{l}(n)$ where $l=k(g)=$ $\sum_{i=1}^{n} a_{i}(g)$, using the cycle monomial $c_{g}=\prod_{i=1}^{n} X_{i}^{a_{i}(g)} \in \mathbb{Z}\left[X_{1}, \ldots, X_{n}\right]$ we get $\sum_{e \in \operatorname{Fix}(g)} w(e)=\prod_{i=1}^{n}\left(\sum_{j=1}^{k} Y_{j}^{i}\right)^{a_{i}(g)}=c_{g}\left(\sum_{j=1}^{k} Y_{j}, \ldots, \sum_{j=1}^{k} Y_{j}^{n}\right) \in R$. 
(17.6) Specialized weights. Specializations of the generic weight algebra give rise to further weight maps, and consequently yield specializations of the above polynomial identity. Keeping the notation of (17.5), the typical specializations are given by replacing some of the indeterminates $Y_{j}$ by 1 , for example:

a) The $\mathbb{Q}$-algebra homomorphism $R \rightarrow \mathbb{Q}: Y_{j} \mapsto 1$ yields the number of patterns as $|G \backslash \operatorname{Maps}(N, K)|=\sum_{f \in S} 1=c_{G}(k, \ldots, k)=k_{G}(k) \in \mathbb{Q}$, where $k_{G}=c_{G}(X, \ldots, X)=\frac{1}{|G|} \cdot \sum_{g \in G} X^{k(g)} \in \mathbb{Q}[X]$ is the cycle-number index. Note that this shows that, varying the cardinality of $K$, the number $k_{G}(k)$ of patterns is given by a polynomial map in $k \in \mathbb{N}_{0}$. In particular, for $G=\{1\}$ we have $k_{\{1\}}=X^{n}$, and thus we recover $|\operatorname{Maps}(N, K)|=k_{\{1\}}(k)=k^{n}$.

More interestingly, let $G=\mathcal{S}_{n}$. Then we have $k_{\mathcal{S}_{n}}=c_{\mathcal{S}_{n}}(X, \ldots, X)=\frac{1}{n !}$. $\sum_{\pi \in \mathcal{S}_{n}} X^{k(\pi)}=\frac{1}{n !} \cdot \sum_{l=0}^{n} s_{n, l} X^{l} \in \mathbb{Q}[X]$. Recalling that $\mathcal{S}_{n} \backslash \operatorname{Maps}(N, K)$ is in bijection with the $n$-multisets $\mathcal{M}_{n}(k)$ on $K$, we get $\frac{1}{n !} \cdot \sum_{l=0}^{n} s_{n, l} k^{l}=$ $c_{\mathcal{S}_{n}}(k, \ldots, k)=\left|\mathcal{M}_{n}(k)\right|=\left(\begin{array}{c}k+n-1 \\ n\end{array}\right)=\frac{1}{n !} \cdot \prod_{i=0}^{n-1}(k+i)$, for all $k \in \mathbb{N}_{0}$, recovering the polynomial identity $\sum_{l=0}^{n} s_{n, l} X^{l}=X^{(n)} \in \mathbb{Q}[X]$.

b) For $k=2$, letting $K=\{0,1\}$, the generic weight algebra becomes $\mathbb{Q}\left[Y_{0}, Y_{1}\right]$, and the $\mathbb{Q}$-algebra homomorphism $\mathbb{Q}\left[Y_{0}, Y_{1}\right] \rightarrow \mathbb{Q}[Y]$ given by $Y_{0} \mapsto 1$ and $Y_{1} \mapsto Y$ yields $\sum_{l \in\{0, \ldots, n\}} \omega_{l} Y^{l}=c_{G}\left(1+Y, \ldots, 1+Y^{n}\right) \in \mathbb{Q}[Y]$, where $\omega_{l}=$ $\left|\left\{f \in S ;\left|f^{-1}(1)\right|=l\right\}\right| \in \mathbb{N}_{0}$, for $l \in\{0, \ldots, n\}$, is the number of patterns with precisely $l$ occurrences of $1 \in K$.

This allows for another interpretation: Viewing the elements of $\operatorname{Maps}(N,\{0,1\})$ as indicator functions yields a bijection $\operatorname{Maps}(N,\{0,1\}) \rightarrow \mathcal{P}(N): f \mapsto f^{-1}(1)$. Moreover, the group $G$ acts on $\mathcal{P}(N)$ via $g: M \mapsto g M:=\{g x \in N ; x \in M\}=$ $\left\{x \in N ; g^{-1} x \in M\right\}$, for all $g \in G$ and $M \subseteq N$; note that $|M|=|g M|$. Then we have $\left({ }^{g} f\right)^{-1}(1)=\left\{x \in N ; f\left(g^{-1}(x)\right)=1\right\}=g\left(f^{-1}(1)\right) \subseteq N$, for all $g \in G$ and $f \in \operatorname{Maps}(N,\{0,1\})$, showing that the above map $\operatorname{Maps}(N,\{0,1\}) \rightarrow \mathcal{P}(N)$ is an isomorphism of $G$-sets.

Hence $\omega_{l}=\left|G \backslash \mathcal{P}_{l}(N)\right| \in \mathbb{N}_{0}$ is the number of $G$-orbits on the set of $l$-subsets of $N$, and thus we get $\sum_{l \in\{0, \ldots, n\}}\left|G \backslash \mathcal{P}_{l}(N)\right| \cdot Y^{l}=c_{G}\left(1+Y, \ldots, 1+Y^{n}\right) \in$ $\mathbb{Q}[Y]$. In particular, the $\mathbb{Q}$-algebra homomorphism $\mathbb{Q}[Y] \rightarrow \mathbb{Q}: Y \mapsto 1$ yields $|G \backslash \mathcal{P}(N)|=\sum_{l \in\{0, \ldots, n\}}\left|G \backslash \mathcal{P}_{l}(N)\right|=c_{G}(2, \ldots, 2)=k_{G}(2)=\frac{1}{|G|} \cdot \sum_{g \in G} 2^{k(g)}$.

Thus for $G=\{1\}$ we recover $\sum_{l \in\{0, \ldots, n\}}\left(\begin{array}{c}n \\ l\end{array}\right) \cdot Y^{l}=\sum_{l \in\{0, \ldots, n\}}\left|\mathcal{P}_{l}(N)\right| \cdot Y^{l}=$ $c_{\{1\}}\left(1+Y, \ldots, 1+Y^{n}\right)=(1+Y)^{n} \in \mathbb{Q}[Y]$; this specializes further yielding $\sum_{l \in\{0, \ldots, n\}}\left(\begin{array}{c}n \\ l\end{array}\right)=|\mathcal{P}(N)|=2^{n}$. For $G=\mathcal{S}_{n}$ we have $\left|G \backslash \mathcal{P}_{l}(N)\right|=1$ for all $l \in\{0, \ldots, n\}$, implying $\sum_{l \in\{0, \ldots, n\}} Y^{l}=c_{\mathcal{S}_{n}}\left(1+Y, \ldots, 1+Y^{n}\right) \in \mathbb{Q}[Y]$, from which by specializing further we obtain $n+1=c_{\mathcal{S}_{n}}(2, \ldots, 2)=k_{\mathcal{S}_{n}}(2)=\frac{1}{n !}$. $\sum_{\pi \in \mathcal{S}_{n}} 2^{k(\pi)}=\frac{1}{n !} \cdot \sum_{l=0}^{n} s_{n, l} 2^{l}$, recovering a special case of the polynomial identity for Stirling numbers of the first kind mentioned above. 


\section{Counting patterns}

(18.1) Example: Necklaces. A necklace with $n \geq 3$ pearls having at most $k \in \mathbb{N}_{0}$ distinct colors is a map $f: N:=\{1, \ldots, n\} \rightarrow\{1, \ldots, k\}=: K$. The set $N$ may be considered as the set of vertices of a regular $n$-gon $\mathcal{R}_{n}$, and necklaces are equivalent if they arise from each other by a symmetry of $\mathcal{R}_{n}$. Hence we consider the dihedral group $D_{2 n} \leq \mathcal{S}_{n}$ acting on the set Maps $(N, K)$ by pre-multiplication, and the equivalence classes are the associated orbits. Thus their number is $\eta_{n}(k):=\left|D_{2 n} \backslash \operatorname{Maps}(N, K)\right|=k_{D_{2 n}}(k)=c_{D_{2 n}}(k, \ldots, k)=$ $\frac{1}{2 n} \cdot \sum_{\pi \in D_{2 n}} k^{k(\pi)} \in \mathbb{N}$.

For example, for $n \in\{3,4\}$ from Table 13 we get the cycle indexes $c_{D_{6}}=$ $\frac{1}{6}\left(X_{1}^{3}+3 X_{2} X_{1}+2 X_{3}\right) \in \mathbb{Q}\left[X_{1}, \ldots, X_{3}\right]$ and $c_{D_{8}}=\frac{1}{8}\left(X_{1}^{4}+2 X_{2} X_{1}^{2}+3 X_{2}^{2}+\right.$ $\left.2 X_{4}\right) \in \mathbb{Q}\left[X_{1}, \ldots, X_{4}\right]$. Thus we have the cycle-number indices $k_{D_{6}}=\frac{1}{6}\left(X^{3}+\right.$ $\left.3 X^{2}+2 X\right) \in \mathbb{Q}[X]$ and $k_{D_{8}}=\frac{1}{8}\left(X^{4}+2 X^{3}+3 X^{2}+2 X\right) \in \mathbb{Q}[X]$, yielding $\eta_{3}(k)=k_{D_{6}}(k)$ and $\eta_{4}(k)=k_{D_{8}}(k)$.

Moreover, letting $\eta_{n, a} \in \mathbb{N}_{0}$ be the number of equivalence classes of necklaces with $n \geq 3$ pearls which are either black or white, such that there are precisely $a \in\{0, \ldots, n\}$ black pearls, we get $\sum_{a \in\{0, \ldots, n\}} \eta_{n, a} Y^{a}=c_{D_{2 n}}(1+$ $\left.Y, 1+Y^{2}, \ldots, 1+Y^{n}\right) \in \mathbb{Q}[Y]$, for $n \in\{3,4\}$ yielding $\sum_{a \in\{0, \ldots, 3\}} \eta_{3, a} Y^{a}=$ $\frac{1}{6}\left((1+Y)^{3}+3\left(1+Y^{2}\right)(1+Y)+2\left(1+Y^{3}\right)\right)=Y^{3}+Y^{2}+Y+1 \in \mathbb{Q}[Y]$ and $\sum_{a \in\{0, \ldots, 4\}} \eta_{4, a} Y^{a}=\frac{1}{8}\left((1+Y)^{4}+2\left(1+Y^{2}\right)(1+Y)^{2}+3\left(1+Y^{2}\right)^{2}+2\left(1+Y^{4}\right)\right)=$ $Y^{4}+Y^{3}+2 Y^{2}+Y+1 \in \mathbb{Q}[Y]$, respectively, where in both cases the numbers $\eta_{n, a}$ are combinatorially obvious.

(18.2) Example: The structure of benzene, revisited. Using the notation of (15.9), finding the number of orbits of $G=G^{(i)}$, for $i \in\{1,2,3\}$, on the set of pairs of distinct elements of $N:=\{1, \ldots, 6\}$, can be rephrased in terms of a pattern counting problem: Letting $K:=\{0,1,2\}$, we have to find the number of patterns ${ }^{G} f \in G \backslash \operatorname{Maps}(N, K)$ such that $\left|f^{-1}(1)\right|=\left|f^{-1}(2)\right|=1$. Thus, specializing the associated generic weight index using the $\mathbb{Q}$-algebra homomorphism $\mathbb{Q}\left[Y_{0}, Y_{1}, Y_{2}\right] \rightarrow \mathbb{Q}\left[Y_{1}, Y_{2}\right]: Y_{0} \mapsto 1, Y_{1} \mapsto Y_{1}, Y_{2} \mapsto Y_{2}$, we have to determine the coefficient $\omega_{[1,1]}$ of the monomial $Y_{1} Y_{2}$ in the specialized weight index. To do so, we compute the cycle index $c\left(X_{1}, \ldots, X_{6}\right) \in \mathbb{Q}\left[X_{1}, \ldots, X_{6}\right]$, and by Polya's Theorem specialize $c\left(1+Y_{1}+Y_{2},, \ldots,, 1+Y_{1}^{6}+Y_{2}^{6}\right) \in \mathbb{Q}\left[Y_{1}, Y_{2}\right]$ :

i) We have $G^{(1)} \cong D_{12}$. It is immediate from the description in (15.3) that $c^{(1)}\left(X_{1}, \ldots, X_{6}\right)=\frac{1}{12} \cdot\left(2 X_{6}+2 X_{3}^{2}+4 X_{2}^{3}+3 X_{2}^{2} X_{1}^{2}+X_{1}^{6}\right) \in \mathbb{Q}\left[X_{1}, \ldots, X_{6}\right]$. In order to determine the coefficient $\omega_{[1,1]}$ we proceed as follows: Expanding $c^{(1)}\left(1+Y_{1}+Y_{2}, \ldots,, 1+Y_{1}^{6}+Y_{2}^{6}\right)$ shows that $Y_{1} Y_{2}$ only occurs in $\left(1+Y_{1}^{2}+\right.$ $\left.Y_{2}^{2}\right)^{2}\left(1+Y_{1}+Y_{2}\right)^{2}$, with coefficient 2 , and in $\left(1+Y_{1}+Y_{2}\right)^{6}$, with coefficient $\frac{6 !}{4 ! \cdot 1 ! \cdot 1 !}=30$. Hence we get $\omega_{[1,1]}=\frac{1}{12} \cdot(3 \cdot 2+30)=3$.

ii) We have $G^{(2)} \cong \mathcal{S}_{3}$. Since $G^{(2)}$ acts transitively on 6 points, this action is equivalent to the regular action of $\mathcal{S}_{3}$. Hence we get $c^{(2)}\left(X_{1}, \ldots, X_{6}\right)=$ $\frac{1}{6} \cdot\left(2 X_{3}^{2}+3 X_{2}^{3}+X_{1}^{6}\right) \in \mathbb{Q}\left[X_{1}, \ldots, X_{6}\right]$, where similarly, $Y_{1} Y_{2}$ only occurs in 
$\left(1+Y_{1}+Y_{2}\right)^{6}$, with coefficient 30 , yielding $\omega_{[1,1]}=\frac{30}{6}=5$.

iii) We have $G^{(3)} \cong \mathcal{S}_{4}$. Since $G^{(3)}$ acts transitively and faithfully on 6 points, this action is equivalent to the action of $\mathcal{S}_{4}$ on the cosets of $\langle(1,2),(3,4)\rangle \cong V_{4}$. Hence we get $c^{(3)}\left(X_{1}, \ldots, X_{6}\right)=\frac{1}{24} \cdot\left(6 X_{4} X_{1}^{2}+8 X_{3}^{2}+6 X_{2}^{3}+3 X_{2}^{2} X_{1}^{2}+X_{1}^{6}\right) \epsilon$ $\mathbb{Q}\left[X_{1}, \ldots, X_{6}\right]$, where similarly $Y_{1} Y_{2}$ only occurs in $\left(1+Y_{1}^{4}+Y_{2}^{4}\right)\left(1+Y_{1}+Y_{2}\right)^{2}$, with coefficient 2 , in $\left(1+Y_{1}^{2}+Y_{2}^{2}\right)^{2}\left(1+Y_{1}+Y_{2}\right)^{2}$, with coefficient 2 , and in $\left(1+Y_{1}+Y_{2}\right)^{6}$, with coefficient 30 , yielding $\omega_{[1,1]}=\frac{1}{24} \cdot(6 \cdot 2+3 \cdot 2+30)=2$. $\sharp$

(18.3) Example: Isomerism of alcohols. An alcohol is a aliphatic hydrocarbon compound having the chemical sum formula $\mathrm{C}_{n} \mathrm{H}_{2 n+1}(\mathrm{OH})$, where $n \in \mathbb{N}$, consisting of a connected skeleton without circles of $n$ carbon atoms, one of which carries a hydroxy group $O H$, and the $2 n+1$ hydrogen atoms are attached to the carbon atoms so that any of the latter is adjacent to precisely 4 neighbors. To determine the number $t_{n} \in \mathbb{N}$ of distinct isomers of alcohols having $n$ carbon atoms (where here we consider stereo isomers as identical), we consider the generating series $t:=\sum_{n>0} t_{n} Y^{n} \in \mathbb{Q}[[Y]]$, where we let $t_{0}:=1$, corresponding to the degenerate alcohol $\bar{H}(\mathrm{OH})$, that is water.

The carbon atom skeleton with the distinguished atom carrying the hydroxy group can be considered as a rooted tree $\Gamma$, that is a connected finite simple graph without circles with an exceptional vertex; the empty graph is a rooted tree, and we let $|\Gamma| \in \mathbb{N}_{0}$ be the number of vertices of $\Gamma$. Note that a tree having $n \geq 1$ vertices has precisely $n-1$ edges, showing that there are indeed precisely $4 n-2(n-1)-1=2 n+1$ free slots for hydrogen atoms. Hence we are interested in the set $\mathcal{T}$ of rooted trees all of whose vertices have valency at most 4 , and whose exceptional vertex has valency at most 3 . Hence we have $\mathcal{T}=\coprod_{n \in \mathbb{N}_{0}} \mathcal{T}_{n}$, where $\mathcal{T}_{n}:=\{\Gamma \in \mathcal{T} ;|\Gamma|=n\}$; in particular, $\mathcal{T}_{0}$ is the singleton set containing the empty graph. Thus we have $t_{n}=\left|\mathcal{T}_{n}\right|$, for $n \in \mathbb{N}_{0}$.

Given $\Gamma \in \mathcal{T}_{n}$, for some $n \geq 1$, allowing for the empty graph as a subtree, there are precisely 3 subtrees $\Gamma_{1}, \Gamma_{2}, \Gamma_{3} \in \mathcal{T}$ attached to the exceptional vertex of $\Gamma$, where the vertex of $\Gamma_{i}$ being attached to the exceptional vertex of $\Gamma$ is designated as exceptional. Since the order of the $\Gamma_{i}$ is irrelevant, we conclude that $\Gamma$ is completely described by the 3 -multiset $\left[\Gamma_{1}, \Gamma_{2}, \Gamma_{3}\right] \subseteq \mathcal{T}$. Thus $\mathcal{T} \backslash \mathcal{T}_{0}$ can be identified with $\mathcal{S}_{3} \backslash \operatorname{Maps}(N, \mathcal{T})$, where $N:=\{1,2,3\}$, by associating $f \in \operatorname{Maps}(N, \mathcal{T})$ with the rooted tree spliced together as described above from $[f(1), f(2), f(3)]$. Hence $t_{n}$ coincides with the number of $\mathcal{S}_{3}$-orbits on the set $\{f \in \operatorname{Maps}(N, \mathcal{T}) ;|f(1)|+|f(2)|+|f(3)|=n-1\}$, for $n \in \mathbb{N}$.

The generic weight algebra for this setting is as follows: Let $\mathcal{Y}:=\left\{Y_{\Gamma} ; \Gamma \in \mathcal{T}\right\}$ be indeterminates. Then for any map $f \in \operatorname{Maps}(N, \mathcal{T})$ the associated weight $w(f)$ is a polynomial of degree 3 in $\mathbb{Q}[\mathcal{Y}]$, but we will have to allow for infinite sums like $\sum_{f \in \operatorname{Maps}(N, \mathcal{T})} w(f)$. Hence the generic weight algebra is defined as the inverse limit $R:=\lim _{n \in \mathbb{N}_{0}}\left\{\mathbb{Q}[\mathcal{Y}] /\left(Y_{\Gamma} ;|\Gamma| \geq n\right)\right\}$, with respect to the natural quotient maps; note that the proof of Polya's Theorem still works for weight maps with values in $R$. We consider the following specialization: Letting 
$Y$ be an indeterminate, the ring of formal power series in $Y$ can be seen as the inverse limit $\mathbb{Q}[[Y]]=\lim _{n \in \mathbb{N}_{0}}\left\{\mathbb{Q}[Y] /\left(Y^{n}\right)\right\}$, with respect to the natural quotient maps. Now, for any $n \in \mathbb{N}_{0}$ there is a $\mathbb{Q}$-algebra homomorphism $\mathbb{Q}[\mathcal{Y}] /\left(Y_{\Gamma} ;|\Gamma| \geq n\right) \rightarrow \mathbb{Q}[Y] /\left(Y^{n}\right): Y_{\Gamma} \mapsto Y^{|\Gamma|}$. Since these maps commute with the respective quotient maps used to define the inverse limits considered, this induces a $\mathbb{Q}$-algebra homomorphism $R \rightarrow \mathbb{Q}[[Y]]: Y_{\Gamma} \mapsto Y^{|\Gamma|}$.

Hence, with respect to this specialization, any map $f \in \operatorname{Maps}(N, \mathcal{T})$ has associated weight $w(f):=Y^{|f(1)|+|f(2)|+|f(3)|} \in \mathbb{Q}[Y]$, we get $\sum_{f \in \mathcal{S}_{3} \backslash \operatorname{Maps}(N, \mathcal{T})} w(f)=$ $\sum_{n \geq 0} t_{n+1} Y^{n}=\frac{1}{Y}(t(Y)-1) \in \mathbb{Q}[[Y]]$. We evaluate the left hand side using Polya's Theorem: The cycle index of $\mathcal{S}_{3}$ is given as $c_{\mathcal{S}_{3}}=\frac{1}{6}\left(X_{1}^{3}+3 X_{2} X_{1}+\right.$ $\left.2 X_{3}\right) \in \mathbb{Q}\left[X_{1}, X_{2}, X_{3}\right]$, see (18.1), and since the sum $\sum_{\Gamma \in \mathcal{T}} Y_{\Gamma} \in R$ specializes to $t=\sum_{n>0} t_{n} Y^{n} \in \mathbb{Q}[[Y]]$, the left hand side equals $c_{\mathcal{S}_{3}}\left(t(Y), t\left(Y^{2}\right), t\left(Y^{3}\right)\right)=$ $\frac{1}{6}\left(t(Y)^{3}+3 t\left(Y^{2}\right) t(Y)+2 t\left(Y^{3}\right)\right) \in \mathbb{Q}[[Y]]$.

Hence we get the functional equation $\frac{1}{Y}(t(Y)-1)=\frac{1}{6}\left(t(Y)^{3}+3 t\left(Y^{2}\right) t(Y)+\right.$ $2 t\left(Y^{3}\right)$ ), or equivalently $t(Y)=1+\frac{Y}{6}\left(t(Y)^{3}+3 t\left(Y^{2}\right) t(Y)+2 t\left(Y^{3}\right)\right)$, allowing to solve recursively for $t_{n}$, for $n \in \mathbb{N}$, given $t_{0}=1$ :

\begin{tabular}{|c|c|c|c|c|c|c|c|c|c|c|c|c|c|c|c|}
\hline$n$ & 0 & 1 & 2 & 3 & 4 & 5 & 6 & 7 & 8 & 9 & 10 & 11 & 12 & 13 & 14 \\
\hline$t$ & 1 & 1 & 1 & 2 & 4 & 8 & 17 & 39 & 89 & 211 & 507 & 1238 & 3057 & 7639 & 19241 \\
\hline
\end{tabular}

Hence in particular there are a unique methanol and a unique ethanol, as well as two propanols, four butanols, eight pentanols, 17 hexanols, and so on.

A closed formula for $t_{n}$ currently is not known. Considering the power series $\sum_{n \geq 0} t_{n} z^{n}$ as a complex function in $z \in \mathbb{C}$, it can be shown that it has (analytically defined) convergence radius $0.35<\rho \sim 0.355181742<0.36$, and that the growth behavior of the $t_{n}$ is described by $\lim _{n \rightarrow \infty}\left(t_{n} \cdot \rho^{n} \cdot n^{\frac{3}{2}}\right)=\gamma$, for some (analytically defined) constant $\gamma \sim 0.5179$, that is $t_{n} \sim\left(\frac{1}{\rho}\right)^{n} \cdot\left(\frac{1}{n}\right)^{\frac{3}{2}}$. Actually, approximations to $\rho$ and $\gamma$ can be found experimentally as follows: Varying $\rho$ within the above bounds, the sequence of the numbers $t_{n} \cdot \rho^{n} \cdot n^{\frac{3}{2}}$, for $n \in\{1, \ldots, 700\}$ say, turns out to be increasing if $\rho$ is chosen too large, while from a certain turning point on it becomes decreasing if $\rho$ is chosen too small, hence we may just try to push this turning point to larger and larger $n$.

b) Similarly, for any $d \geq 2$, we may count the set $\mathcal{T}^{(d)}$ of $d$-ary rooted trees, that is rooted trees all of whose vertices have valency at most $d$ and whose exceptional vertex has valency at most $d-1$, using the action of the symmetric group $\mathcal{S}_{d}$ on $\operatorname{Maps}\left(\{1, \ldots, d\}, \mathcal{T}^{(d)}\right)$.

For example, for $d=2$ we get the cycle index $c_{\mathcal{S}_{2}}=\frac{1}{2}\left(X_{1}^{2}+X_{2}\right) \in \mathbb{Q}\left[X_{1}, X_{2}\right]$, and hence the associated generating series $\tau:=\sum_{n \geq 0} \tau_{n} Y^{n} \in \mathbb{Q}[[Y]]$, where $\tau_{n}:=$ $\left|\left\{\Gamma \in \mathcal{T}^{(2)} ;|\Gamma|=n\right\}\right|$ for $n \in \mathbb{N}_{0}$, fulfills the functional equation $\frac{1}{Y}(\tau(Y)-1)=$ $\frac{1}{2}\left(\tau(Y)^{2}+\tau\left(Y^{2}\right)\right)$. Using $\tau_{0}=1$, the latter recursively yields the WedderburnEtherington numbers as follows:

\begin{tabular}{|l||rrrrrrrrrrrrrrr|}
\hline$n$ & 0 & 1 & 2 & 3 & 4 & 5 & 6 & 7 & 8 & 9 & 10 & 11 & 12 & 13 & 14 \\
\hline$\tau_{n}$ & 1 & 1 & 1 & 2 & 3 & 6 & 11 & 23 & 46 & 98 & 207 & 451 & 983 & 2179 & 4850 \\
\hline
\end{tabular}


A closed formula for the $\tau_{n}$ currently is not known. Considering the power series $\sum_{n \geq 0} \tau_{n} z^{n}$ as a complex function in $z \in \mathbb{C}$, it can be shown that it has convergence radius $\rho \sim 0.402697504$, and that the growth behavior of the $\tau_{n}$ is described by $\lim _{n \rightarrow \infty}\left(\tau_{n} \cdot \rho^{n-1} \cdot n^{\frac{3}{2}}\right)=\gamma \sim 0.7916$.

(18.4) Example: The card game SET. a) The card game SET was invented by Falco [1974] as a translation of observables used in a study of the genetics of epilepsy in dogs, German shepherds to be precise. The translation consists of four attributes, each of which can assume three distinct values, of the symbols on specially tailored playing cards; this amounts to a total of $3^{4}=81$ cards:

\begin{tabular}{|r|ccc|}
\hline attribute & 0 & 1 & 2 \\
\hline \hline quantity & three & one & two \\
color & green & red & purple \\
shading & open & striped & solid \\
shape & diamond & squiggle & oval \\
\hline
\end{tabular}

Given a subset of cards, a 'set' in the sense of this game is a subset of three cards, such that with respect to any of the attributes, independently, the three cards either all have the same value or are pairwise distinct. 'Set'-free subsets of cards are also called caps, where those which are not properly contained in a larger cap are called maximal caps.

The game then is played as follows: Twelve cards are laid out face-up on the table. The player first detecting a 'set' collects these three cards, and the latter are replaced by three new ones. If there is no 'set' detected, then three more cards are laid out. This is iterated until the deck is empty, and the remaining cards laid out form a cap. The player with the most 'sets' detected wins.

This leads, at least, to the following questions: Firstly, with respect to all subsets of cards: When are two subsets of cards equivalent, with respect to the 'set' property? How many inequivalent $k$-subsets of cards are there? (In particular, for $k=12$ ?) Secondly, with respect to caps: What is the largest $k=k_{\max }$ such that there is a $k$-cap of cards? (In particular, is $k_{\max } \geq 12$ ?) For $k \leq k_{\max }$, how many inequivalent $k$-caps of cards are there? What is the proportion of the $k$-caps amongst all $k$-subsets of cards? How do maximal caps look like? (In particular, for $k=k_{\max }$ ?)

In order to attack this algebraically, the values of the attributes are associated with $\mathbb{F}_{3}:=\{0,1,2\}$, as is already indicated above. Hence the cards can be identified with the vectors in $\mathbb{F}_{3}^{4}$. Then the condition that, for a fixed attribute, three values either are all the same or are pairwise distinct translates into saying that their sum vanishes. Hence a 'set' of cards just is a 3 -subset $\left\{v, v^{\prime}, v^{\prime \prime}\right\} \subseteq \mathbb{F}_{3}^{4}$ such that $v+v^{\prime}+v^{\prime \prime}=0 \in \mathbb{F}_{3}^{4}$. Moreover, the admissible symmetries are the bijections on $\mathbb{F}_{3}^{4}$ mapping 'sets' to 'sets'.

The condition of being a 'set' can be rephrased as follows: If $\left\{v, v^{\prime}, v^{\prime \prime}\right\}$ is a 'set', we have $v^{\prime}=v+\left(v^{\prime}-v\right)$ and $v^{\prime \prime}=-\left(v+v^{\prime}\right)=v+2\left(v^{\prime}-v\right)$, thus 
$\left\{v, v^{\prime}, v^{\prime \prime}\right\}=v+\mathbb{F}_{3} \cdot\left(v^{\prime}-v\right)$, where $0 \neq\left(v^{\prime}-v\right) \in \mathbb{F}_{3}^{4}$. Conversely, for any $0 \neq u \in \mathbb{F}_{3}^{4}$, the 3-subset $v+\mathbb{F}_{3} \cdot u \subseteq \mathbb{F}_{3}^{4}$ fulfills $v+(v+u)+(v+2 u)=0$, hence is a 'set'. Thus 'sets' are just the 'lines' in $\mathbb{F}_{3}^{4}$ in the following geometrical sense:

b) Letting $d \in \mathbb{N}$ and $\mathbb{F}_{q}$ be the field with $q$ elements, we consider the affine space with underlying points $V:=\mathbb{F}_{q}^{d}$, whose lines are the subsets $v+\langle u\rangle_{\mathbb{F}_{q}} \subseteq$ $V$, for some $v \in V$ and $0 \neq u \in V$. Then the associated symmetries (in the sense of affine geometry) are given by the affine general linear group $A:=\mathrm{AGL}_{d}(q) \cong T \rtimes \mathrm{GL}_{d}(q)$, where $\mathrm{GL}_{d}(q) \leq A$ is the subgroup of affine maps fixing the origin, that is the subgroup of $\mathbb{F}_{q}$-linear maps, and $T \cong V$ is the normal subgroup of translations; hence we have $|A|=q^{d} \cdot \prod_{i=0}^{d-1}\left(q^{d}-q^{i}\right)$. Then $A$ acts faithfully on $V$ via $[t, g] \cdot v:=t+g v$, for $v \in V$ and $g \in \operatorname{GL}_{d}(q)$ and $t \in T$. Embedding $A \rightarrow \mathrm{GL}_{d+1}(q)$ via $[t, g] \mapsto\left[\begin{array}{c|c}g & t \\ \hline \cdot & 1\end{array}\right]$, and identifying $v \in V$ with the extended vector $\left[v^{\operatorname{tr}} \mid 1\right]^{\operatorname{tr}} \in \mathbb{F}_{q}^{d+1}$ we get $\left[\begin{array}{c|c}g & t \\ \hline \cdot & 1\end{array}\right] \cdot\left[\begin{array}{l}v \\ \hline 1\end{array}\right]=\left[\begin{array}{c}g v+t \\ \hline 1\end{array}\right]$, implying that affine maps can be considered as $\mathbb{F}_{q}$-linear maps.

This paves the way to find the cycle index of $A$; note that using the structure of $A$ as a semidirect product it is possible to use the well-understood conjugacy classes of $\mathrm{GL}_{d}(q)$ to obtain a conceptual description of the conjugacy classes of $A$, but instead we pursue a purely computational approach: Considering the action of $A$ on extended vectors, we obtain a faithful permutation representation, which can be used to determine its conjugacy classes, together with their cardinality, and the cycle type of its elements. Given the cycle index $c_{A} \in \mathbb{Q}\left[X_{1}, \ldots, X_{q^{d}}\right]$ of $A$, the number of orbits of $A$ on the $k$-subsets of $V$, for $k \in\left\{0, \ldots, q^{d}\right\}$, is readily given by the coefficient of $Y^{k}$ in $c_{A}\left(1+Y, \ldots, 1+Y^{q^{d}}\right) \in \mathbb{Q}[Y]$.

However, it is computationally much more difficult to find a transversal of the $A$-orbits on the $k$-subsets of $V$. Moreover, the picture changes completely when it comes to finding the number of $A$-orbits on the $k$-caps in $V$, where Polya's Theorem does not at all help: Actually, neither $k_{\max }$ is known, apart from a very small cases, nor the growth behavior of the number of orbits, for fixed $k$ and growing $d$, let alone explicit formulae for them, are known. But since any subset of a cap again is a cap, it it possible to use recursion with respect to $k$ to explicitly find a transversal of the $A$-orbits on $k$-caps, and hence in particular their number, and consequently $k_{\max }$, for a few non-trivial cases.

c) Now let $q:=3$. For $d=1$ we get $A \cong \mathbb{F}_{3} \rtimes \mathbb{F}_{3}^{*} \cong \mathcal{S}_{3}$, acting naturally, which (obviously) yields $c_{A}\left(1+Y, \ldots, 1+Y^{3}\right)=Y^{3}+Y^{2}+Y+1 \in \mathbb{Q}[Y]$. For $d \in\{2,3,4\}$, where $|A|=432$ and $|A|=303264$ and $|A|=1965150720$, respectively, proceeding as described above yields $c_{A}\left(1+Y, \ldots, 1+Y^{9}\right)=Y^{9}+$ $Y^{8}+Y^{7}+2 Y^{6}+2 Y^{5}+2 Y^{4}+2 Y^{3}+Y^{2}+Y+1 \in \mathbb{Q}[Y]$ for $d=2$, and $c_{A}\left(1+Y, \ldots, 1+Y^{27}\right)=Y^{27}+Y^{26}+Y^{25}+2 Y^{24}+3 Y^{23}+5 Y^{22}+10 Y^{21}+$ $16 Y^{20}+28 Y^{19}+47 Y^{18}+68 Y^{17}+91 Y^{16}+114 Y^{15}+127 Y^{14}+127 Y^{13}+114 Y^{12}+$ $91 Y^{11}+68 Y^{10}+47 Y^{9}+28 Y^{8}+16 Y^{7}+10 Y^{6}+5 Y^{5}+3 Y^{4}+2 Y^{3}+Y^{2}+Y+1 \in \mathbb{Q}[Y]$ for $d=3$, and $c_{A}\left(1+Y, \ldots, 1+Y^{81}\right) \in \mathbb{Q}[Y]$ for $d=4$ is given in Table 19 ; 
hence there are 41407 inequivalent 12-subsets of cards. Note that, while for $d \leq 3$ transversals of the $A$-orbits on the $k$-subsets of $V$ are easily found for $k \in\left\{1, \ldots, q^{d}\right\}$, for $d=4$ this is computationally tractable only up to $k \sim 14$.

As for caps and the $A$-orbits thereon, the knowledge is very poor. The current state of the art for $k_{\max }$ is as follows:

\begin{tabular}{|l||c|c|c|c|c|c|}
\hline$d$ & 1 & 2 & 3 & 4 & 5 & 6 \\
\hline$k_{\max }$ & 2 & 4 & 9 & 20 & 45 & $\{112,113,114\}$ \\
\hline
\end{tabular}

Transversals and the number of $A$-orbits are known for $d \in\{1, \ldots, 4\}$ and all $k \in\left\{0, \ldots, k_{\max }\right\}$, from explicit computations, see Table 20 . This verifies the above results on $k_{\max }$ in this range. In particular, it turns out that there always is a single $A$-orbit of caps of cardinality $k_{\max }$; there is no a-priori-reason known why this should be the case. Moreover, for $d=4$ we indicate the proportion of $k$-caps amongst all $k$-subsets of $V$; a cap of cardinality $k_{\max }=20$ (actually the lexicographically smallest one) is reproduced in Table 21, together with its translation into real cards according to the table given earlier. 
Table 19: Number of $\mathrm{AGL}_{4}(3)$-orbits on subsets of $\mathbb{F}_{3}^{4}$.

$$
\begin{gathered}
Y^{81}+ \\
Y^{80}+Y^{79}+ \\
2 Y^{78}+3 Y^{77}+6 Y^{76}+ \\
15 Y^{75}+34 Y^{74}+105 Y^{73}+ \\
384 Y^{72}+1658 Y^{71}+8135 Y^{70}+ \\
41407 Y^{69}+205211 Y^{68}+963708 Y^{67}+ \\
4231059 Y^{66}+17295730 Y^{65}+65807588 Y^{64}+ \\
233346408 Y^{63}+772518828 Y^{62}+2392611091 Y^{61}+ \\
6946116261 Y^{60}+18937468347 Y^{59}+48568206996 Y^{58}+ \\
117356752981 Y^{57}+267548687984 Y^{56}+576222904363 Y^{55}+ \\
1173737365919 Y^{54}+2263568972663 Y^{53}+4136780036942 Y^{52}+ \\
7170309576688 Y^{51}+11796184561289 Y^{50}+18431386920534 Y^{49}+ \\
27367649303603 Y^{48}+38636503940897 Y^{47}+51883126670392 Y^{46}+ \\
66294936428615 Y^{45}+80628826002618 Y^{44}+93359571424793 Y^{43}+ \\
102934827016066 Y^{42}+108081525023972 Y^{41}+108081525023972 Y^{40}+ \\
102934827016066 Y^{39}+93359571424793 Y^{38}+80628826002618 Y^{37}+ \\
66294936428615 Y^{36}+51883126670392 Y^{35}+38636503940897 Y^{34}+ \\
27367649303603 Y^{33}+18431386920534 Y^{32}+11796184561289 Y^{31}+ \\
7170309576688 Y^{30}+4136780036942 Y^{29}+2263568972663 Y^{28}+ \\
1173737365919 Y^{27}+576222904363 Y^{26}+267548687984 Y^{25}+ \\
117356752981 Y^{24}+48568206996 Y^{23}+18937468347 Y^{22}+ \\
6946116261 Y^{21}+2392611091 Y^{20}+772518828 Y^{19}+ \\
233346408 Y^{18}+65807588 Y^{17}+17295730 Y^{16}+ \\
4231059 Y^{15}+963708 Y^{14}+205211 Y^{13}+ \\
41407 Y^{12}+8135 Y^{11}+1658 Y^{10}+ \\
384 Y^{9}+105 Y^{8}+34 Y^{7}+ \\
25 Y^{3}+6 Y^{5}+3 Y^{4}+ \\
Y^{2}+1 \\
\end{gathered}
$$


Table 20: Number of $\mathrm{AGL}_{d}(3)$-orbits on $k$-caps.

\begin{tabular}{|r||r|r|r|r|r|}
\hline$k$ & 1 & 2 & 3 & 4 & prop. \\
\hline \hline 1 & 1 & 1 & 1 & 1 & \\
2 & & 1 & 1 & 1 & \\
3 & & 1 & 1 & 1 & 0.99 \\
4 & & 1 & 2 & 2 & 0.95 \\
5 & & & 2 & 3 & 0.88 \\
6 & & & 3 & 7 & 0.76 \\
7 & & & 2 & 11 & 0.62 \\
8 & & & 3 & 33 & 0.45 \\
9 & & & 1 & 91 & 0.30 \\
10 & & & & 267 & 0.17 \\
\hline
\end{tabular}

\begin{tabular}{|r||r|r|r||r|l|}
\hline$k$ & 1 & 2 & 3 & 4 & prop. \\
\hline \hline 11 & & & & 670 & $8 \cdot 10^{-2}$ \\
12 & & & & 1437 & $3 \cdot 10^{-2}$ \\
13 & & & & 2225 & $1 \cdot 10^{-2}$ \\
14 & & & & 2489 & $2 \cdot 10^{-3}$ \\
15 & & & & 1756 & $4 \cdot 10^{-4}$ \\
16 & & & & 748 & $3 \cdot 10^{-5}$ \\
17 & & & & 143 & $1 \cdot 10^{-6}$ \\
18 & & & & 20 & $1 \cdot 10^{-8}$ \\
19 & & & & 1 & $9 \cdot 10^{-12}$ \\
20 & & & & 1 & $1 \cdot 10^{-13}$ \\
\hline
\end{tabular}

Table 21: A 20-cap for $d=4$.

\begin{tabular}{|l|l|}
\hline$[0,0,0,0]$ & three-green-open-diamond \\
{$[0,0,0,1]$} & three-green-open-squiggle \\
{$[0,0,1,0]$} & three-green-striped-diamond \\
{$[0,0,1,1]$} & three-green-striped-squiggle \\
{$[0,1,0,0]$} & three-red-open-diamond \\
{$[0,1,0,1]$} & three-red-open-squiggle \\
{$[0,1,1,0]$} & three-red-striped-diamond \\
{$[0,1,1,1]$} & three-red-striped-squiggle \\
{$[1,0,0,0]$} & one-green-open-diamond \\
{$[1,0,0,1]$} & one-green-open-squiggle \\
{$[1,0,1,2]$} & one-green-striped-oval \\
{$[1,0,2,2]$} & one-green-solid-oval \\
{$[1,1,0,2]$} & one-red-open-oval \\
{$[1,2,0,2]$} & one-purple-open-oval \\
{$[2,0,1,2]$} & two-green-striped-oval \\
{$[2,1,0,2]$} & two-red-open-oval \\
{$[2,1,1,0]$} & two-red-striped-diamond \\
{$[2,1,1,1]$} & two-red-striped-squiggle \\
{$[2,1,2,2]$} & two-red-solid-oval \\
{$[2,2,1,2]$} & two-purple-striped-oval \\
\hline
\end{tabular}




\section{Exercises and references}

\section{Exercises for Part I (in German)}

(19.1) Aufgabe: Potenzmenge.

Es seien $M$ eine beliebige Menge und $\mathcal{P}(M)$ ihre Potenzmenge. Man zeige: Es gibt keine Surjektion $M \rightarrow \mathcal{P}(M)$.

Hinweis. Für $f: M \rightarrow \mathcal{P}(M)$ surjektiv betrachte man $\{x \in M ; x \notin f(x)\}$.

(19.2) Aufgabe: Potenzmengen endlicher Mengen.

Für $n \in \mathbb{N}_{0}$ bestimme man (ohne die Benutzung von Binomialkoeffizienten) die Anzahl der Teilmengen von $\{1, \ldots, n\}$ mit gerader bzw. ungerader Kardinalität.

(19.3) Aufgabe: Aufzählung von Potenzmengen.

Es sei $n \in \mathbb{N}_{0}$. Zur Gray-Aufzählung aller Teilmengen von $\{1, \ldots, n\}$ werden diese als $n$-Tupel mit Einträgen in $\{0,1\}$ dargestellt. Ist $\mathcal{G}(n)=\left[x_{1}, \ldots, x_{2^{n}}\right]$ die Aufzählung für $n$, so sei $\mathcal{G}(n+1):=\left[0 x_{1}, 0 x_{2}, \ldots, 0 x_{2^{n}}, 1 x_{2^{n}}, 1 x_{2^{n}-1}, \ldots, 1 x_{1}\right]$, wobei $\mathcal{G}(0):=[[]]$ die Liste mit dem leeren Tupel sei. Man zeige:

a) Je zwei benachbarte Tupel in $\mathcal{G}(n)$ unterscheiden sich an genau einer Stelle.

b) Für $0 \leq k \leq n$ sei $\mathcal{G}_{k}(n)$ die Unterfolge von $\mathcal{G}(n)$ der Tupel mit genau $k$ Einträgen 1. Man zeige: Je zwei benachbarte Tupel in $\mathcal{G}_{k}(n)$ unterscheiden sich an genau zwei Stellen.

(19.4) Aufgabe: Parlamentswahlen.

Das Parlament eines Landes habe $n \in \mathbb{N}$ Sitze, und es seien die rote, die grüne und die gelbe Partei vertreten. Wieviele mögliche Sitzverteilungen gibt es, so daß keine Partei die absolute Mehrheit hat?

(19.5) Aufgabe: Kartenmischen.

Ein Stapel von 52 üblichen Spielkarten wird gemischt. Wieviele Ergebnisse gibt es, bei denen sowohl die oberste als auch die unterste Karte ein Ass ist?

(19.6) Aufgabe: Fakultäten.

Man zeige: Jede natürliche Zahl $n \in \mathbb{N}_{0}$ hat eine eindeutige Darstellung der Form $n=\sum_{k \geq 1} a_{k} \cdot k$ ! mit $a_{k} \in\{0, \ldots, k\}$.

(19.7) Aufgabe: Teilmengen ohne benachbarte Elemente.

a) Für $n \in \mathbb{N}_{0}$ und $k \in \mathbb{N}_{0}$ seien $a_{k}(n) \in \mathbb{N}_{0}$ die Anzahl der $k$-elementigen Teilmengen $M \subseteq\{1, \ldots, n\}$ mit $|x-y| \geq 2$ für alle $x \neq y \in M$, sowie $a(n)=$ $\sum_{k=0}^{n} a_{k}(n)$ die Anzahl aller solcher Teilmengen von $\{1, \ldots, n\}$.

Man zeige kombinatorisch: Es gelten $a_{k}(n)=\left(\begin{array}{c}n-k+1 \\ k\end{array}\right)$ sowie $a(n)=F_{n+2}$, die $(n+2)$-te Fibonacci-Zahl. (Dies ist also ein kombinatorischer Beweis der Identität $\sum_{k=0}^{n}\left(\begin{array}{c}n-k+1 \\ k\end{array}\right)=F_{n+2}$. $)$ 
b) Nun sei $j \in \mathbb{N}$. Man bestimme die Anzahl der $k$-elementigen Teilmengen $M \subseteq\{1, \ldots, n\}$ mit $|x-y| \geq j$ für alle $x \neq y \in M$.

\section{(19.8) Aufgabe: Multimengen.}

Es seien $n \in \mathbb{N}_{0}$ und $k \in \mathbb{N}_{0}$. Man zeige: Es gilt $\left|\mathcal{M}_{k}(n+1)\right|=\left|\mathcal{M}_{n}(k+1)\right|$; dazu gebe man einen algebraischen und einen kombinatorischen Beweis an.

\section{(19.9) Aufgabe: Teiler.}

Für $n \in \mathbb{N}$ sei $\tau(n):=|\{d \in \mathbb{N} ; d \mid n\}| \in \mathbb{N}$ die Anzahl der Teiler von $n$. Man zeige: Die Anzahl $\tau(n)$ ist genau dann ungerade, wenn $n$ eine Quadratzahl ist.

\section{(19.10) Aufgabe: Gefangenenbefreiung.}

Der Gefängniswärter eines Gefängnisses mit 100 Zellen will einige Gefangenen freilassen, indem er folgendes Verfahren anwendet: Zunächst schließt er alle Türen auf, dann der Reihe nach jede zweite wieder zu, danach ändert er den Zustand jeder dritten Tür von offen nach geschlossen und umgekehrt, und so fährt er mit jeder vierten, fünften, bis hundertsten Tür fort. Am Ende dürfen die Gefangenen gehen, deren Tür offen ist. Welche Gefangenen sind das?

\section{(19.11) Aufgabe: Warteschlangen.}

Die Personen $\{1, \ldots, n\}$, für $n \in \mathbb{N}$, mögen eine Warteschlage bilden, so daß sich, mit Ausnahme der vordersten Person, vor jeder Person $i$ die Person $i-1$ oder $i+1$ befinde. Wieviele mögliche Warteschlangen gibt es?

\section{(19.12) Aufgabe: Fallende Faktorielle.}

a) Es sei $n \in \mathbb{N}_{0}$. Man zeige: Es gilt $(2 X)_{(2 n)}=2^{2 n} X_{(n)}\left(X-\frac{1}{2}\right)_{(n)} \in \mathbb{Z}[X]$.

b) Daraus folgere man: Es gilt $\left(\begin{array}{c}-\frac{1}{2} \\ n\end{array}\right)=\left(-\frac{1}{4}\right)^{n} \cdot\left(\begin{array}{c}2 n \\ n\end{array}\right)$.

\section{(19.13) Aufgabe: Binomialkoeffizienten.}

Es sei $n \in \mathbb{N}_{0}$. Man zeige:

a) Es gilt Unimodalität $\left(\begin{array}{l}n \\ 0\end{array}\right)<\left(\begin{array}{l}n \\ 1\end{array}\right)<\cdots<\left(\begin{array}{c}n \\ \left\lfloor\frac{n}{2}\right\rfloor\end{array}\right)=\left(\begin{array}{c}n \\ \left\lceil\frac{n}{2}\right\rceil\end{array}\right)>\cdots>\left(\begin{array}{c}n \\ n-1\end{array}\right)>\left(\begin{array}{l}n \\ n\end{array}\right)$.

b) Es gelten $\sum_{k=0}^{n} k \cdot\left(\begin{array}{l}n \\ k\end{array}\right)=n \cdot 2^{n-1}$ und $\sum_{k=0}^{n} k^{2} \cdot\left(\begin{array}{l}n \\ k\end{array}\right)=n(n+1) \cdot 2^{n-2}$.

c) Es gilt $\sum_{k=0}^{n}\left(\begin{array}{c}n \\ k\end{array}\right)^{2}=\left(\begin{array}{c}2 n \\ n\end{array}\right)$.

\section{(19.14) Aufgabe: Identitäten für Binomialkoeffizienten.}

Es seien $m \leq n \in \mathbb{N}_{0}$. Man zeige:

a) Es gilt $\left(\begin{array}{c}n \\ m\end{array}\right)\left(\begin{array}{c}m \\ k\end{array}\right)=\left(\begin{array}{c}n \\ k\end{array}\right)\left(\begin{array}{c}n-k \\ m-k\end{array}\right)$ und $\sum_{k=0}^{m}\left(\begin{array}{c}n \\ k\end{array}\right)\left(\begin{array}{c}n-k \\ m-k\end{array}\right)=2^{m} \cdot\left(\begin{array}{c}n \\ m\end{array}\right)$. Welche kombinatorische Interpretation hat diese Identität?

b) Es gilt $\sum_{k=0}^{m}(-1)^{k} \cdot\left(\begin{array}{l}n \\ k\end{array}\right)\left(\begin{array}{c}n-k \\ m-k\end{array}\right)= \begin{cases}1, & \text { falls } m=0 \\ 0, & \text { falls } m \geq 1 .\end{cases}$ 
c) Es gilt $\sum_{k=0}^{m}(-1)^{k} \cdot\left(\begin{array}{c}n \\ k\end{array}\right)\left(\begin{array}{c}n \\ m-k\end{array}\right)=\left\{\begin{aligned} 0, & \text { falls } m \text { ungerade, } \\ (-1)^{\frac{m}{2}} \cdot\left(\begin{array}{c}n \\ \frac{m}{2}\end{array}\right), & \text { falls } m \text { gerade. }\end{aligned}\right.$

Hinweis zu c). Man betrachte $(X+1)^{n}(X-1)^{n}=\left(X^{2}-1\right)^{n} \in \mathbb{Z}[X]$.

(19.15) Aufgabe: Pascal-Dreieck.

Es seien $n \in \mathbb{N}_{0}$ und $k \in \mathbb{N}_{0}$. Man zeige: Für partielle Antidiagonalsummen gilt $\sum_{i=0}^{k}\left(\begin{array}{c}n+i \\ k-i\end{array}\right)=F_{n+k+1}$, die $(n+k+1)$-te Fibonacci-Zahl.

(19.16) Aufgabe: Verschobenes Pascal-Dreieck.

Man betrachte das verschobene Pascal-Dreieck, in dem die $n+1$ nicht-verschwindenden Einträge in Zeile $n \in \mathbb{N}_{0}$ in den Spalten $2 n, \ldots, 3 n$ stehen, und die Einträge eingerahmt werden, die Vielfache von $n$ sind:

\begin{tabular}{|c|c|c|c|c|c|c|c|c|c|c|c|c|c|c|c|}
\hline$n \backslash k$ & $\begin{array}{ll}0 & 1\end{array}$ & 2 & 3 & 4 & 5 & 6 & 7 & 8 & 9 & 10 & 11 & 12 & 13 & 14 & 15 \\
\hline$\overline{0}$ & $\overline{1}$ & & & & & & & & & & & & & & \\
\hline 1 & & \begin{tabular}{|l|}
1 \\
\end{tabular} & 1 & & & & & & & & & & & & \\
\hline 2 & & & & 1 & 2 & 1 & & & & & & & & & \\
\hline 3 & & & & & & 1 & 3 & 3 & 1 & & & & & & \\
\hline 4 & & & & & & & & 1 & 4 & 6 & \begin{tabular}{|l|}
4 \\
\end{tabular} & 1 & & & \\
\hline 5 & & & & & & & & & & 1 & 5 & \begin{tabular}{|l|}
10 \\
\end{tabular} & 10 & 5 & 1 \\
\hline
\end{tabular}

Man zeige: Es ist $k \geq 2$ genau dann eine Primzahl, wenn alle nicht-verschwindenden Einträge in Spalte $k$ eingerahmt sind.

(19.17) Aufgabe: Gitterwege.

Für $m, n \in \mathbb{N}_{0}$ betrachte man ein Gitter mit den Kantenlängen $m$ und $n$. Durch Klassifikation von Wegen gebe man kombinatorische Beweise der folgenden Identitäten an: Für $k \in \mathbb{N}_{0}$ gilt $\sum_{i=0}^{k}\left(\begin{array}{c}n+i \\ i\end{array}\right)=\left(\begin{array}{c}n+k+1 \\ k\end{array}\right)$ und $\left(\begin{array}{c}m+n \\ k\end{array}\right)=\sum_{i=0}^{k}\left(\begin{array}{c}m \\ i\end{array}\right)\left(\begin{array}{c}n \\ k-i\end{array}\right)$.

Hinweis. Man betrachte das erste Eintreffen auf der rechten Vertikalen bzw. den Schnittpunkt mit einer Antidiagonalen.

(19.18) Aufgabe: Schachbrett.

Für $n \in \mathbb{N}_{0}$ betrachte man ein $(n \times n)$-Schachbrett. Man bestimme die Anzahl der kürzesten Wege, auf denen ein König von der linken unteren zur rechten oberen Ecke gelangen kann.

(19.19) Aufgabe: Bertrands Problem.

Zwei Kandidaten A und B erhalten bei einer Wahl $a>b \in \mathbb{N}_{0}$ Stimmen. Man zeige, daß die Stimmzettel auf genau $\frac{a-b}{a+b} \cdot\left(\begin{array}{c}a+b \\ a\end{array}\right)$ Weisen geordnet werden können, so daß bei der sukzessiven Auszählung A stets mehr Stimmen als B hat.

Hinweis. Man betrachte geeignete Gitterwege. 
(19.20) Aufgabe: Kleiner Satz von Fermat.

Es seien $n \in \mathbb{N}$ und $p \in \mathbb{N}$ eine Primzahl. Man zeige: Es gilt $n^{p} \equiv n(\bmod p)$; dazu gebe man einen algebraischen und einen kombinatorischen Beweis an.

\section{(19.21) Aufgabe: Folgen von Teilmengen.}

Es seien $n \in \mathbb{N}_{0}$ und $k \in \mathbb{N}_{0}$. Man bestimme jeweils die Anzahl der Folgen $\left[N_{1}, \ldots, N_{k}\right]$ von Teilmengen $N_{i} \subseteq N:=\{1, \ldots, n\}$ mit:

a) $N_{1} \subseteq N_{2} \subseteq \cdots \subseteq N_{k}$

b) $N_{i} \cap N_{j}=\emptyset$ für alle $i \neq j \in\{1, \ldots, k\}$;

c) $N_{1} \cup N_{2} \cup \cdots \cup N_{k}=N$.

\section{(19.22) Aufgabe: Stirling-Zahlen zweiter Art.}

a) Für $n \in \mathbb{N}_{0}$ zeige man: Es gilt $S_{n, 0}<S_{n, 1}<\cdots<S_{n, m_{n}-1} \leq S_{n, m_{n}}>\cdots>$ $S_{n, n-1}>S_{n, n}$, für ein $m_{n} \in\{0, \ldots, n\}$ mit $m_{n}=m_{n-1}+\epsilon$, wobei $\epsilon \in\{0,1\}$.

b) Für $n \geq 2$ zeige man: Es gilt $S_{n, n-2}=\left(\begin{array}{l}n \\ 3\end{array}\right)+3 \cdot\left(\begin{array}{l}n \\ 4\end{array}\right)$.

c) Es seien $n \in \mathbb{N}_{0}$ und $k \in \mathbb{N}_{0}$. Man zeige: Es gilt $S_{n+1, k+1}=\sum_{i=0}^{n}\left(\begin{array}{c}n \\ i\end{array}\right) S_{i, k}$; dazu gebe man einen algebraischen und einen kombinatorischen Beweis an.

d) Man bestimme die Anzahl der Tupel $\left[a_{1}, \ldots, a_{n}\right] \in \mathbb{N}^{n}$, für $n \in \mathbb{N}_{0}$, deren Einträge genau die Zahlen $\{1, \ldots, k\}$ sind (eventuell mehrfach), so daß der erste Eintrag $i$ vor dem ersten Eintrag $i+1$ liegt, für alle $i \in\{1, \ldots, k-1\}$.

\section{(19.23) Aufgabe: Bell-Zahlen.}

a) Es sei $n \in \mathbb{N}_{0}$. Man zeige: Es gilt $B_{n+1}=\sum_{k=0}^{n}\left(\begin{array}{l}n \\ k\end{array}\right) B_{k}$.

b) Es sei $n \in \mathbb{N}$. Man zeige: Es gibt genau $B_{n-1}$ Partitionen von $\{1, \ldots, n\}$, deren sämtliche Blöcke keine zwei aufeinanderfolgende Zahlen enthalten.

\section{(19.24) Aufgabe: Teile in Kompositionen.}

a) Es sei $n \in \mathbb{N}$. Man zeige: Die Gesamtanzahl aller Teile in allen Kompositionen von $n$ ist gleich $(n+1) \cdot 2^{n-2}$.

b) Es sei $k \in \mathbb{N}$ mit $k<n$. Man zeige: In allen Kompositionen von $n$ kommt der Teil $k$ insgesamt genau $(n-k+3) \cdot 2^{n-k-2}$ mal vor.

(19.25) Aufgabe: Kompositionen.

a) Es seien $n \in \mathbb{N}_{0}$ und $k \in \mathbb{N}_{0}$. Man bestimme jeweils die Anzahl der Tupel $\left[a_{1}, \ldots, a_{k}\right]$ positiver bzw. nicht-negativer ganzer Zahlen mit $\sum_{i=1}^{k} a_{i} \leq n$.

b) Unter Benutzung der Fibonacci-Zahlen zeige man:

i) Es gibt genau $F_{n-1}$ Kompositionen von $n$ in Teile $\geq 2$;

ii) es gibt genau $F_{n+1}$ Kompositionen von $n$ in Teile $\leq 2$;

iii) es gibt genau $F_{n}$ Kompositionen von $n$ in ungerade Teile. 
(19.26) Aufgabe: Partitionen.

Es sei $p_{n} \in \mathbb{N}$ die Anzahl der Partitionen von $n \in \mathbb{N}_{0}$. Man zeige: Es gilt $p_{n+1}-2 p_{n+1}+p_{n} \geq 0$.

Hinweis. Es ist $p_{n}-p_{n-1}$ die Anzahl der Partitionen von $n$ ohne den Teil 1.

\section{(19.27) Aufgabe: Partitionen mit mehrfachen Teilen.}

Es sei $n \in \mathbb{N}_{0}$. Für eine Partition $\lambda=\left[\lambda_{1}, \ldots, \lambda_{k}\right]=\left[n^{a_{n}(\lambda)}, \ldots, 1^{a_{1}(\lambda)}\right] \in P_{k}(n)$ mit $k \in \mathbb{N}_{0}$ Teilen seien $\alpha(\lambda):=\prod_{i=1}^{k} \lambda_{i} \in \mathbb{N}$ und $\beta(\lambda):=\prod_{i=1}^{n} a_{i}(\lambda) ! \in \mathbb{N}$. Man zeige: Es gilt $\prod_{\lambda \in P(n)} \alpha(\lambda)=\prod_{\lambda \in P(n)} \beta(\lambda)$.

Hinweis. Für $j \in \mathbb{N}$ setze $\operatorname{man} b_{j}(\lambda):=\left|\left\{i \in\{1, \ldots, n\} ; a_{i}(\lambda) \geq j\right\}\right| \in \mathbb{N}_{0}$ und zeige $\sum_{\lambda \in P(n)} a_{j}(\lambda)=\sum_{\lambda \in P(n)} b_{j}(\lambda)$.

(19.28) Aufgabe: Partitionen mit vielen Teilen.

Es sei $k \in \mathbb{N}_{0}$. Man zeige: Es gibt $n_{k} \geq k$ und $\rho_{k} \in \mathbb{N}$ mit $p_{n, n-k}=\rho_{k}$ für alle $n \geq n_{k}$. Man bestimme $\rho_{k}$, und gebe das kleinstmögliche $n_{k}$ an.

\section{(19.29) Aufgabe: Partitionen mit drei Teilen.}

Es sei $n \in \mathbb{N}_{0}$. Man zeige: Ist $n \equiv a(\bmod 6)$, wobei $a \in\{0, \ldots, 5\}$, so ist die Anzahl der Partitionen von $n$ in drei Teile gegeben als $p_{a, 3}(n) \in \mathbb{N}_{0}$, wobei

$$
p_{a, 3}(X)= \begin{cases}\frac{X^{2}}{12}, & \text { für } a=0, \\ \frac{X^{2}-1}{12}, & \text { für } a \in\{1,5\}, \\ \frac{X^{2}-4}{12}, & \text { für } a \in\{2,4\}, \\ \frac{X^{2}+3}{12}, & \text { für } a=3 .\end{cases}
$$

(19.30) Aufgabe: Aufzählung von Permutationen.

Für $n \in \mathbb{N}_{0}$ sei $\mathcal{S}_{n}=\{[1,2, \ldots, n], \ldots,[n, n-1, \ldots, 1]\}$ in lexikographischer Anordnung gegeben. Für $\pi=\left[\pi_{1}, \ldots, \pi_{n}\right] \in \mathcal{S}_{n}$ sei $\alpha_{n}(\pi)+1 \in\{1, \ldots, n$ ! $\}$ die Position von $\pi$ in $\mathcal{S}_{n}$, etwa $\alpha_{n}([1,2, \ldots, n])=0$ und $\alpha_{n}([n, n-1, \ldots, 1])=n !-1$.

a) Man gebe einen Algorithmus an, der zu einer Permutation $\pi \in \mathcal{S}_{n}$ den Nachfolger berechnet, also die Permutation $\sigma \in \mathcal{S}_{n}$ mit $\alpha_{n}(\sigma)=\alpha_{n}(\pi)+1$.

b) Für $n \geq 1$ zeige man: Es gilt $\alpha_{n}(\pi)=\left(\pi_{1}-1\right)(n-1)$ ! $+\alpha_{n-1}\left(\pi^{\prime}\right)$, wobei $\pi^{\prime}$ aus $\pi$ entsteht, indem man $\pi_{1}$ entfernt und alle $\pi_{i}>\pi_{1}$ durch $\pi_{i}-1$ ersetzt.

c) Für $l \in\{0, \ldots, n !-1\}$ bestimme man die Permutation $\pi \in \mathcal{S}_{n}$ mit $\alpha_{n}(\pi)=l$.

(19.31) Aufgabe: Bell-Zahlen und Permutationen.

Für $n \in \mathbb{N}$ seien $\mathcal{I}_{n}^{+} \subseteq \mathcal{S}_{n}$ und $\mathcal{I}_{n}^{-} \subseteq \mathcal{S}_{n}$ die Mengen aller Permutationen $\pi=$ $\left[\pi_{1}, \ldots, \pi_{n}\right] \in \mathcal{S}_{n}$ mit folgender Eigenschaft: Es gibt kein $i<j \in\{1, \ldots, n-1\}$ mit $\pi_{i}<\pi_{j}<\pi_{j+1}$. bzw. mit $\pi_{i}<\pi_{j+1}<\pi_{j}$.

a) Man zeige: Es gilt $\left|\mathcal{I}_{n}^{+}\right|=B_{n}=\left|\mathcal{I}_{n}^{-}\right|$.

b) Man beschreibe die Menge $\mathcal{I}_{n}^{+} \cap \mathcal{I}_{n}^{-} \subseteq \mathcal{S}_{n}$. 
(19.32) Aufgabe: Inversionen.

Es seien $n \in \mathbb{N}_{0}$ und $\pi=\left[\pi_{1}, \ldots, \pi_{n}\right] \in \mathcal{S}_{n}$. Ein Paar $\pi_{i}>\pi_{j}$, wobei $i<j$ ist, heißt eine Inversion von $\pi$. Es sei $l(\pi) \in \mathbb{N}_{0}$ die Anzahl der Inversionen von $\pi$, und für $i \in\{1, \ldots, n\}$ sei $\iota_{i} \in \mathbb{N}_{0}$ die Anzahl der $j \in\{i+1, \ldots, n\}$, sodaß $[j, i]$ eine Inversion von $\pi$ ist; die Folge $\left[\iota_{1}, \ldots, \iota_{n}\right]$ heißt die Inversionstafel von $\pi$.

a) Es sei $I_{n, k} \in \mathbb{N}_{0}$ die Anzahl der Permutationen in $\mathcal{S}_{n}$ mit $k \in \mathbb{N}_{0}$ Inversionen. Man zeige: Es gilt $I_{n, 0}=1$, und $I_{n, k}=I_{n-1, k}+I_{n, k-1}$ für $1 \leq k<n$.

b) Man zeige: Es gilt $I_{n, k}=0$ für $k>\left(\begin{array}{l}n \\ 2\end{array}\right)$, und $I_{n, k}=I_{n,\left(\begin{array}{l}n \\ 2\end{array}\right)-k}$ für $k \leq\left(\begin{array}{l}n \\ 2\end{array}\right)$, sowie $\sum_{k=0}^{\left(\begin{array}{c}n \\ 2\end{array}\right)}(-1)^{k} I_{n, k}=0$ für $n \geq 2$. Außerdem gilt $l(\pi)=l\left(\pi^{-1}\right)$.

c) Man zeige: Für die Einträge von Inversionstafeln gilt $\iota_{i} \in\{0, \ldots, n-i\}$, für alle $i \in\{1, \ldots, n\}$. Umgekehrt ist jede Folge $\left[\iota_{1}, \ldots, \iota_{n}\right]$ mit $\iota_{i} \in\{0, \ldots, n-i\}$, für alle $i \in\{1, \ldots, n\}$, die Inversionstafel genau einer Permutation in $\mathcal{S}_{n}$.

\section{(19.33) Aufgabe: Stirling-Zahlen erster Art.}

a) Für $n \in \mathbb{N}_{0}$ zeige man: Es gilt $s_{n, 0}<s_{n, 1}<\cdots<s_{n, m_{n}-1} \leq s_{n, m_{n}}>\cdots>$ $s_{n, n-1}>s_{n, n}$, für ein $m_{n} \in\{0, \ldots, n\}$ mit $m_{n}=m_{n-1}+\epsilon$, wobei $\epsilon \in\{0,1\}$.

b) Für $n \geq 2$ zeige man: Es gilt $s_{n, n-2}=2 \cdot\left(\begin{array}{l}n \\ 3\end{array}\right)+3 \cdot\left(\begin{array}{l}n \\ 4\end{array}\right)$.

c) Es seien $n \in \mathbb{N}_{0}$ und $k \in \mathbb{N}_{0}$. Man zeige: Es gilt $s_{n+1, k+1}=\sum_{i=0}^{n}\left(\begin{array}{c}i \\ k\end{array}\right) s_{n, i}$; dazu gebe man einen algebraischen und einen kombinatorischen Beweis an.

(19.34) Aufgabe: Fixpunktfreie Permutationen.

Für $n \geq 2$ sei $D^{+}(n) \in \mathbb{N}_{0}$ die Anzahl der fixpunktfreien Permutationen in der alternierenden Gruppe $\mathcal{A}_{n}:=\left\{\pi \in \mathcal{S}_{n} ; \operatorname{sgn}(\pi)=1\right\}<\mathcal{S}_{n}$. Man zeige: Es gilt $D^{+}(n)=(-1)^{n-1}(n-1)+\frac{n !}{2} \cdot \sum_{k=0}^{n-2} \frac{(-1)^{k}}{k !}$.

(19.35) Aufgabe: Die Theatergarderobe.

Die Garderobenpersonal des 'Theater 99' ist unaufmerksam. (Das Theater ist übrigens nach der Anzahl seiner Sitzplätze benannt.) Nach der Vorstellung werden die Mäntel zufällig an die Besucher zurückgegeben. Wie groß ist die Wahrscheinlichkeit (ungefähr), daß dabei keiner den eigenen Mantel erhält?

(19.36) Aufgabe: Derangement-Zahlen.

Für $n \in \mathbb{N}$ betrachte man die folgenden Zahlen: Man beginne mit 1, subtrahiere 1 , multipliziere mit 2 , addiere 1 , multipliziere mit 3 , subtrahiere $1, \ldots$, multipliziere mit $n$ und addiere $(-1)^{n}$. Welche bekannte Folge erhält man?

(19.37) Aufgabe: Türme von Hanoi.

Auf drei senkrechten Stäben $A, B$ und $C$ sind $n \in \mathbb{N}_{0}$ Scheiben unterschiedlichen Durchmessers gestapelt, so daß jede nicht zuunterst liegende Scheibe auf einer mindestens ebenso großen Scheibe liegt. Durch einen Zug kann man die oberste Scheibe eines Stabes auf einen anderen Stab bewegen, falls dabei diese Bedingung nicht verletzt wird. Zunächst befinden sich alle Scheiben auf Stab $A$. 
a) Wieviele Züge sind nötig, die Scheiben nach Stab $B$ zu bewegen, wenn die Scheiben paarweise verschiedenen Durchmesser haben?

b) Wieviele Züge sind nötig, die Scheiben nach Stab $B$ zu bewegen, wenn die Scheiben paarweise verschiedenen Durchmesser haben, und nur Züge zwischen den Stäben $A$ und $C$ sowie $B$ und $C$ erlaubt sind?

c) Wieviele Züge sind nötig, die Scheiben nach Stab $B$ zu bewegen, wenn Scheiben gleichen Durchmessers jeweils paarweise vorkommen?

(19.38) Aufgabe: Summation.

Für $n \in \mathbb{N}_{0}$ berechne man $\sum_{k=1}^{n} \frac{2 k+1}{k(k+1)} \in \mathbb{Q}$ und $\sum_{k=1}^{n}(-1)^{k} \cdot k \in \mathbb{Q}$ sowie $\sum_{k=1}^{n}(-1)^{k} \cdot k^{2} \in \mathbb{Q}$.

\section{(19.39) Aufgabe: Reelle Differentiation.}

Für $a \in \mathbb{R} \backslash\{1\}$ und $n \in \mathbb{N}_{0}$ seien $g_{a}(n):=\sum_{k=1}^{n} a^{k}$ und $f_{a}(n):=\sum_{k=1}^{n} k \cdot a^{k}$. Man gebe geschlossene Formeln für $g_{a}(n)$ und $f_{a}(n)$ an. Welche bekannte Formel erhält man für $f_{2}(n)$ ? Was passiert für $a=1$ ?

\section{(19.40) Aufgabe: Potenzsummen.}

Für $k \geq 1$ sei $s_{k} \in \mathbb{Q}[X]$, so daß $s_{k}(n)=\sum_{i=0}^{n-1} i^{k}$ für alle $n \in \mathbb{N}_{0}$.

a) Man zeige: Es gilt $s_{k}(1-X)=(-1)^{k+1} s_{k}(X) \in \mathbb{Q}[X]$.

b) Man bestimme $s_{k}(1) \in \mathbb{Q}$, sowie $s_{k}\left(\frac{1}{2}\right) \in \mathbb{Q}$ für $k$ gerade.

(19.41) Aufgabe: Partielle Summation.

Es sei $n \in \mathbb{N}_{0}$, und für $k \in \mathbb{N}_{0}$ sei $h_{k} \in \mathbb{Q}$ die $k$-te harmonische Zahl.

a) Man berechne $\sum_{k=1}^{n} \frac{h_{k}}{k} \in \mathbb{Q}$ und $\sum_{k=1}^{n} \frac{h_{k}}{(k+1)(k+2)} \in \mathbb{Q}$.

b) Man berechne $\sum_{k=1}^{n} h_{k}^{2} \in \mathbb{Q}$.

c) Für $m \in \mathbb{N}_{0}$ berechne man $\sum_{k=1}^{n}\left(\begin{array}{c}k \\ m\end{array}\right) \cdot h_{k} \in \mathbb{Q}$ und $\sum_{k=1}^{n}(-1)^{k}\left(\begin{array}{c}m \\ k\end{array}\right) \cdot h_{k} \in \mathbb{Q}$.

\section{(19.42) Aufgabe: Diskrete Integration.}

Man gebe eine geschlossene Formel für $\sum_{k=0}^{n} \frac{(-1)^{k}}{\left(\begin{array}{l}n \\ k\end{array}\right)}$, wobei $n \in \mathbb{N}_{0}$, an.

(19.43) Aufgabe: Summen fallender Faktorieller.

Für $n \in \mathbb{N}_{0}$ sei $f_{n}:=\sum_{k=0}^{n-1} n_{(k)} \in \mathbb{N}$. Man bestimme eine Rekursionsformel und daraus eine geschlossene Formel für $f_{n}$.

(19.44) Aufgabe: Binomialinversion.

Man zeige: Es gibt eine Folge $\left[a_{n} \in \mathbb{Q} ; n \in \mathbb{N}_{0}\right]$, so daß $n !=\sum_{k>0} a_{k} n_{(k)}$, für alle $n \in \mathbb{N}_{0}$, gilt. Man gebe eine geschlossene Formel für die Zahlen $a_{n}$ an. 
(19.45) Aufgabe: Lineare Inversion.

a) Man zeige, daß die Basisfolgen $\left[X_{(n)} \in \mathbb{Q}[X] ; n \in \mathbb{N}_{0}\right]$ und $\left[(-1)^{n} X^{(n)} \in\right.$ $\left.\mathbb{Q}[X] ; n \in \mathbb{N}_{0}\right]$ durch die Lah-Zahlen $L_{n, k}:=(-1)^{n} \cdot \frac{n !}{k !} \cdot\left(\begin{array}{l}n-1 \\ k-1\end{array}\right)$, für $n \in \mathbb{N}_{0}$ und $k \in \mathbb{N}_{0}$, zusammenhängen.

Daraus folgere man die Lah-Inversion: Sind $\left[x_{n} \in \mathbb{Q} ; n \in \mathbb{N}_{0}\right]$ und $\left[y_{n} \in\right.$ $\left.\mathbb{Q} ; n \in \mathbb{N}_{0}\right]$, so gilt genau dann $y_{n}=\sum_{k=0}^{n} L_{n, k} x_{k} \in \mathbb{Q}$, für alle $n \in \mathbb{N}_{0}$, wenn $x_{n}=\sum_{k=0}^{n} L_{n, k} y_{k} \in \mathbb{Q}$, für alle $n \in \mathbb{N}_{0}$, gilt.

b) Unter Benutzung der Basisfolge $\sum_{k=0}^{\left\lfloor\frac{n}{2}\right\rfloor}(-1)^{k} \frac{n}{n-k}\left(\begin{array}{c}n-k \\ k\end{array}\right) X^{n-2 k} \in \mathbb{Q}[X]$, für $n \in \mathbb{N}_{0}$, beweise man die Tschebyscheff-Inversion: Sind $\left[x_{n} \in \mathbb{Q} ; n \in \mathbb{N}_{0}\right]$ und $\left[y_{n} \in \mathbb{Q} ; n \in \mathbb{N}_{0}\right]$, so gilt genau dann $y_{n}=\sum_{k=0}^{\left\lfloor\frac{n}{2}\right\rfloor}\left(\begin{array}{l}n \\ k\end{array}\right) x_{n-2 k} \in \mathbb{Q}$, für alle $n \in \mathbb{N}_{0}$, wenn $x_{n}=\sum_{k=0}^{\left\lfloor\frac{n}{2}\right\rfloor}(-1)^{k} \frac{n}{n-k}\left(\begin{array}{c}n-k \\ k\end{array}\right) y_{n-2 k} \in \mathbb{Q}$, für alle $n \in \mathbb{N}_{0}$, gilt.

\section{Exercises for Part II (in German)}

(20.1) Aufgabe: Endliche partiell geordnete Mengen.

a) Man bestimme bis auf Isomorphie alle endlichen partiell geordneten Mengen der Kardinalität $\leq 4$.

b) Man bestimme bis auf Isomorphie alle Verbände der Kardinalität $\leq 6$.

(20.2) Aufgabe: Partiell geordnete Mengen.

Es sei $X$ eine partiell geordnete Menge.

a) Man zeige: Es gibt eine Menge $N$ und eine Teilmenge $\mathcal{X} \subseteq \mathcal{P}(N)$, so daß $X \cong \mathcal{X}$ als partiell geordnete Mengen. Ist zudem $X$ endlich, so kann auch $N$ endlich gewählt werden.

b) Es seien $X$ endlich und $f: X \rightarrow X$ eine ordnungserhaltende Bijektion, das heißt, für $x \leq y \in X$ ist stets auch $f(x) \leq f(y)$. Man zeige: Die Abbildung $f$ ist ein Automorphismus. Kann man auf die Endlichkeitsvoraussetzung verzichten?

b) Es sei $X$ ein Verband, und $f: X \rightarrow X$ eine ordnungserhaltende Bijektion, die auch kleinste obere Schranken und größte untere Schranken erhält. Man zeige: Die Abbildung $f$ ist ein Automorphismus.

(20.3) Aufgabe: Endliche Verbände.

a) Es sei $X$ eine endliche partiell geordnete Menge, die ein Eins-Element besitzt, und für die je zwei Elemente eine größte untere Schranke besitzen. Man zeige: $X$ ist ein Verband. Man formuliere und beweise die dazu duale Aussage.

b) Es sei $X$ ein endlicher Verband. Man zeige die Äquivalenz der folgenden Aussagen: i) $X$ ist graduiert, und für die Längen-Funktion gilt $d(x)+d(y) \geq$ $d(x \vee y)+d(x \wedge y)$, für alle $x, y \in X$. ii) Für alle $x, y \in X$ mit $x \wedge y<x$ und $x \wedge y<y$ gilt stets auch $x<x \vee y$ und $y \ll x \vee y$. 
(20.4) Aufgabe: Maximale Ketten.

Es sei $X$ eine lokal-endliche partiell geordnete Menge, und für $x, y \in X$ sei $m(x, y) \in \mathbb{N}_{0}$ die Anzahl der maximalen Ketten zwischen $x$ und $y$.

a) Es sei $\eta \in \mathcal{A}(X)$ definiert durch $\eta(x, y):=1$ für $x<\cdot y$, und $\eta(x, y):=0$ sonst. Man zeige: Die Funktion $\delta-\eta \in \mathcal{A}(X)$ ist invertierbar, und für $x, y \in X$ gilt $(\delta-\eta)^{-1}(x, y)=m(x, y)$.

b) Es seien $X$ zudem graduiert, und $f \in \mathcal{A}(X)$ definiert durch $f(x, y):=\frac{m(x, y)}{l(x, y) !}$ für $x \leq y$, und $f(x, y):=0$ sonst. Man zeige: Die Funktion $f \in \mathcal{A}(X)$ ist invertierbar, und für $x \leq y$ gilt $f^{-1}(x, y)=(-1)^{l(x, y)} \cdot f(x, y)$.

\section{(20.5) Aufgabe: Dominanz-Ordnung.}

Man betrachte die durch $\unlhd$ gegebene partielle Ordnung auf $P(n)$, für $n \in \mathbb{N}_{0}$.

a) Man zeige: Die durch $\unlhd$ gegebene partielle Ordnung auf $P(n)$ ist ein Verband. Für welche $n \in \mathbb{N}_{0}$ ist $\unlhd$ eine Ordnung? Für welche $n \in \mathbb{N}_{0}$ ist $\unlhd$ graduiert? Man zeichne die Hasse-Diagramme von $\unlhd$ für $n \leq 8$.

b) Für $\lambda=\left[\lambda_{1}, \ldots, \lambda_{n}\right] \vdash n$ und $\mu=\left[\mu_{1}, \ldots, \mu_{n}\right] \vdash n$ schreibe $\mu \leq \lambda$, falls es $k \in\{1, \ldots, n\}$ gibt mit $\mu_{i}=\lambda_{i}$ für alle $i \in\{1, \ldots, k-1\}$, und $\mu_{k}<\lambda_{k}$. Man zeige: Dies definiert eine Ordnung, die lexikographische Ordnung, auf $P(n)$, die die partielle Ordnung $\unlhd$ verfeinert.

\section{Exercises for Part III (in German)}

\section{(21.1) Aufgabe: Möbius-Funktion.}

Es sei $X$ eine endliche partiell geordnete Menge mit Null-Element $\underline{0}$ und EinsElement $\underline{1}$. Für die Möbius-Funktion $\mu$ von $X$ zeige man:

a) Es gilt $\sum_{x, y \in X, x \leq y} \mu(x, y)=1$.

b) Es gilt $\sum_{k \geq 0} \sum_{\underline{0}=x_{0}<x_{1}<\cdots<x_{k}=\underline{1}}(-1)^{k} \cdot \prod_{i=1}^{k} \mu\left(x_{i-1}, x_{i}\right)=1$.

\section{(21.2) Aufgabe: Möbius-Inversion.}

Es seien $X$ ein endlicher Verband $X$ mit Null-Element $\underline{0}$, sowie $g: X \rightarrow \mathbb{N}_{0}: x \mapsto$ $|\{y \in X ; x \leq y\}|$ und $f_{k}: X \rightarrow \mathbb{N}_{0}: x \mapsto \mid\left\{\mid x_{1}, \ldots, x_{k}\right] \in X^{k} ; x_{1} \wedge \cdots \wedge x_{k}=$ $x\} \mid$, für $k \in \mathbb{N}_{0}$. Man zeige: Es gilt $f_{k}(x)=\sum_{x \in X} \mu(\underline{0}, x) \cdot g(x)^{k}$; dazu gebe man sowohl einen Beweis mittels Möbius-Inversion an, also auch einen, der die Möbius-Algebra benutzt.

(21.3) Aufgabe: Zahlentheoretische Möbius-Funktion.

Für $n \in \mathbb{N}$ sei $\mathcal{U}_{n}:=\left\{d \in \mathbb{N} ; d \mid n, \operatorname{ggT}\left(d, \frac{n}{d}\right)=1\right\}$ partiell geordnet durch Teilbarkeit. Man zeige: $\mathcal{U}_{n}$ ist ein Verband, und bestimme seine Möbius-Funktion.

(21.4) Aufgabe: Inklusion-Exklusion.

Es seien $m \leq k \leq n$. Man zeige: Es gilt $\left(\begin{array}{c}n-m \\ k-m\end{array}\right)=\sum_{i=0}^{m}(-1)^{i} \cdot\left(\begin{array}{c}m \\ i\end{array}\right)\left(\begin{array}{c}n-i \\ k\end{array}\right)$. 
Hinweis. Es ist $\left(\begin{array}{c}n-m \\ k-m\end{array}\right)$ die Anzahl der $k$-elementigen Teilmengen einer $n$ elementigen Menge, die eine feste $m$-elementige Teilmenge umfassen.

\section{(21.5) Aufgabe: Dezimalzahlen.}

Wieviele Zahlen mit genau $k \in \mathbb{N}$ Dezimalstellen gibt es, in deren Dezimaldarstellung keine benachbarten Ziffern gleich sind?

\section{(21.6) Aufgabe: Primzahlen.}

a) Unter Benutzung von Inklusion-Exklusion gebe man ein Verfahren an, um aus der Kenntnis aller Primzahlen $\leq \sqrt{n}$, wobei $n \in \mathbb{N}$, die Anzahl der Primzahlen $\leq n$ bestimmen kann, ohne diese zu berechnen. Als Anwendung bestimme man die Anzahl der Primzahlen $\leq 100$ aus der Kenntnis der Primzahlen $\{2,3,5,7\}$, und verifiziere das Ergebnis explizit.

b) Für $n \in \mathbb{N}$ sei $\varphi(n):=|\{k \in\{1, \ldots, n\} ; \operatorname{ggT}(k, n)=1\}| \in \mathbb{N}$ die EulerFunktion. Mittels Inklusion-Exklusion zeige man: Sind $\left\{p_{1}, \ldots, p_{l}\right\}$ die Primteiler von $n$, so gilt $\frac{\varphi(n)}{n}=\prod_{i=1}^{l}\left(1-\frac{1}{p_{i}}\right)$.

(21.7) Aufgabe: Partitionen in ungerade und verschiedene Teile. Für $n \in \mathbb{N}_{0}$ seien $O(n)$ und $D(n)$ die Mengen aller Partitionen von $n$ in lauter ungerade bzw. in paarweise verschiedene Teile. Man gebe eine explizite Bijektion $O(n) \rightarrow D(n)$ an.

Hinweis. Man betrachte die folgende Abbildung $O(n) \rightarrow D(n): \lambda \mapsto \mu$ : Hat $\lambda$ den Teil $i$ genau $a$-mal, so habe $\mu$ den Teil $i \cdot 2^{b}$ genau dann, wenn $2^{b}$ in der 2-adischen Darstellung von $a$ vorkommt.

(21.8) Aufgabe: Menage-Zahlen.

Es sei $u_{n}:=\sum_{k=0}^{n} \frac{(-1)^{k} \cdot(n-k) ! \cdot 2 n}{2 n-k} \cdot\left(\begin{array}{c}2 n-k \\ k\end{array}\right) \in \mathbb{N}_{0}$ die Menage-Zahl, für $n \geq 2$.

a) Man zeige: Für $n \geq 4$ gilt $(n-2) u_{n}=n(n-2) u_{n-1}+n u_{n-2}+4 \cdot(-1)^{n+1}$.

b) Man zeige: Es gilt $\lim _{n \rightarrow \infty} \frac{u_{n}}{n !}=\frac{1}{e^{2}}$.

\section{(21.9) Aufgabe: Folgen mit verschiedenen Nachbarn.}

Es sei $\mathcal{X}_{n}$ die Menge aller Folgen der Länge $2 n$ über einer Menge $X$ der Kardinalität $n \in \mathbb{N}_{0}$, die jedes Element von $X$ genau zweimal enthalten, und so daß benachbarte Folgenglieder jeweils verschieden sind. Man zeige: Es gilt $\left|\mathcal{X}_{n}\right|=\sum_{k=0}^{n} \frac{(-1)^{n-k} \cdot(n+k) !}{2^{k}} \cdot\left(\begin{array}{l}n \\ k\end{array}\right)$.

(21.10) Aufgabe: Äquivalenzklassen von Permutationen.

Es sei $n \in \mathbb{N}_{0}$. Zwei Permutationen der Menge $X_{n}:=\left\{a_{1}, \ldots, a_{n}, b_{1}, \ldots, b_{n}\right\}$, geschrieben als Listen, heißen äquivalent, falls sie durch Vertauschung zweier benachbarter Einträge $a_{i} b_{i}$ oder $b_{i} a_{i}$, für ein $i \in\{1, \ldots, n\}$, auseinander hervorgehen. Man zeige: Die davon erzeugte Äquivalenzrelation auf $\mathcal{S}_{X_{n}}$ besteht aus genau $\sum_{i=0}^{n}(-1)^{i}\left(\begin{array}{c}n \\ i\end{array}\right)(2 n-i)$ ! Äquivalenzklassen. 


\section{(21.11) Aufgabe: Paarungen.}

An einem runden Tisch sitzen $2 n$ Personen, wobei $n \in \mathbb{N}_{0}$. Auf wieviele Weisen kann man daraus $n$ Paare bilden, wenn sich darunter keine zwei benachbarte Personen befinden dürfen?

(21.12) Aufgabe: Zykel mit eingeschränkten Nachbarn.

Für $n \in \mathbb{N}_{0}$ sei $f(n) \in \mathbb{N}_{0}$ die Anzahl der $n$-Zykel $\left(a_{1}, \ldots, a_{n}\right) \in \mathcal{S}_{n}$, so daß $a_{i+1} \not \equiv a_{i}+1(\bmod n)$, für alle $i \in\{1, \ldots, n\}$, wobei $a_{n+1}:=a_{1}$.

a) Man zeige: Es gilt $f(n)=(-1)^{n}+\sum_{i=0}^{n-1}(-1)^{i}\left(\begin{array}{c}n \\ i\end{array}\right)(n-1-i)$ !, und folgere daraus $\lim _{n \rightarrow \infty} \frac{f(n)}{(n-1) !}=\frac{1}{e}$.

b) Für die $n$-te Derangement-Zahl zeige man: Es gilt $D_{n}=f(n)+f(n+1)$.

\section{(21.13) Aufgabe: Zykellängen.}

a) Für $n \in \mathbb{N}_{0}$ und $k \in \mathbb{N}$ sei $f_{k}(n) \in \mathbb{N}_{0}$ die Anzahl der Permutationen in $\mathcal{S}_{n}$, die keinen Zykel der Länge $k$ enthalten. Man zeige: Es gilt $f_{k}(n)=n ! \cdot \sum_{i=0}^{\left\lfloor\frac{n}{k}\right\rfloor} \frac{(-1)^{i}}{i ! \cdot k^{i}}$, und folgere daraus $\lim _{n \rightarrow \infty} \frac{f_{k}(n)}{n !}=e^{-\frac{1}{k}}$.

b) Für $n \in \mathbb{N}_{0}$ sei $f(n) \in \mathbb{N}_{0}$ die Anzahl der Permutationen in $\mathcal{S}_{n}$, die einen Zykel der Länge $>\left\lfloor\frac{n}{2}\right\rfloor$ enthalten. Man zeige: Es gilt $\lim _{n \rightarrow \infty} \frac{f(n)}{n !}=\ln (2)$.

\section{(21.14) Aufgabe: Numerierte Kisten.}

In einem Raum sind 100 verschlossene Kisten fest montiert. Der Boden jeder Kiste trägt eine Zahl zwischen 1 und 100, wobei jede dieser Zahlen genau einmal vorkommt. Außerhalb des Raumes sind 100 Personen, numeriert von 1 bis 100 . Ohne miteinander zu sprechen gehen die Personen nun einzeln in den Raum, öffnen jeweils genau 50 Kisten, schauen hinein, schließen sie und kommen heraus.

Die Wahrscheinlichkeit, daß eine Person zufällig die Kiste mit ihrer Nummer findet, ist $\frac{1}{2}$. Die Wahrscheinlichkeit, daß alle Personen jeweils ihre Nummer finden, ist also $\left(\frac{1}{2}\right)^{100} \sim 10^{-30}$. Können die Personen eine Strategie vereinbaren, so daß die Wahrscheinlichkeit, daß alle Personen ihre Nummer finden, $>\frac{3}{10}$ ist?

\section{Exercises for Part IV (in German)}

(22.1) Aufgabe: Erzeugende Funktionen vom Dirichlet-Typ.

Für eine Folge $\left[f_{n} \in \mathbb{C} ; n \in \mathbb{N}_{0}\right]$ heißt die formale Reihe der Form $f(s):=$ $\sum_{n \geq 1} \frac{f_{n}}{n^{s}}$, wobei $s \in \mathbb{C}$, die zugehörige Dirichlet-Reihe. Dann kann $\mathcal{D}:=$ $\operatorname{Abb}(\mathbb{N}, \mathbb{C})$ als die Menge der Dirichlet-Reihen aufgefaßt werden, und ist ein $\mathbb{C}$ Vektorraum mit punktweiser Addition und Skalarmultiplikation. Auf $\mathcal{D}$ werde eine Multiplikation wie folgt definiert: Für $f=\sum_{n \geq 1} \frac{f_{n}}{n^{s}}$ und $g=\sum_{n \geq 1} \frac{g_{n}}{n^{s}}$ sei $(f g)(s) \in \mathcal{D}$ gegeben durch $(f g)(s):=\sum_{n \geq 1}\left(\sum_{d \mid n} \frac{f_{d} g_{\frac{n}{d}}}{d}\right) \frac{1}{n^{s}}$.

a) Man zeige: $\mathcal{D}$ ist eine kommutative $\mathbb{C}$-Algebra mit Eins $\frac{1}{1^{s}}+\sum_{n \geq 2} \frac{0}{n^{s}} \in \mathcal{D}$, und $f(s)=\sum_{n \geq 1} \frac{f_{n}}{n^{s}} \in \mathcal{D}$ ist genau dann invertierbar, wenn $f_{1} \neq 0$ ist. 
b) Es sei $\zeta(s):=\sum_{n \geq 1} \frac{1}{n^{s}} \in \mathcal{D}$ die Riemann-Funktion. Man zeige: Es ist $\zeta^{-1}(s):=\sum_{n \geq 1} \frac{\mu(n)}{n^{s}}$, wobei $\mu$ die zahlentheoretische Möbius-Funktion ist.

\section{(22.2) Aufgabe: Harmonische Zahlen.}

Für $n \in \mathbb{N}$ seien $h_{n}:=\sum_{i=1}^{n} \frac{1}{i} \in \mathbb{Q}$ die $n$-te harmonische Zahl, und $h_{0}:=0$.

a) Man bestimme die erzeugende Funktion $\sum_{n \geq 0} h_{n} X^{n} \in \mathbb{Q}[[X]]$.

b) Für $n \in \mathbb{N}_{0}$ berechne man $\sum_{k=0}^{n} h_{k} h_{n-k} \in \mathbb{Q}$.

\section{(22.3) Aufgabe: Erzeugende Funktionen.}

Es sei $\left[f_{n} \in \mathbb{Z} ; n \in \mathbb{N}_{0}\right]$ die durch $f_{0}:=1$ und $\sum_{k=0}^{n} f_{k} f_{n-k}=1$, für $n \geq 1$, rekursiv definierte Folge. Man gebe eine geschlossene Formel für $f_{n}$ an.

\section{(22.4) Aufgabe: Linear rekursive Folgen.}

Es seien $d \in \mathbb{N}$ sowie $q_{1}, \ldots, q_{d} \in \mathbb{C}$ mit $q_{d} \neq 0$, und $F:=\left[f_{n} \in \mathbb{C} ; n \in \mathbb{N}_{0}\right]$ die durch Startwerte $f_{0}, \ldots, f_{d-1} \in \mathbb{C}$ und $f_{n+d}+\sum_{i=1}^{d} q_{i} f_{n+d-i}=0$, für alle $n \in \mathbb{N}_{0}$, definierte linear rekursive Folge.

a) Man zeige: Die Folge $F$ kann eindeutig zu einer Folge $\left[f_{n} \in \mathbb{C} ; n \in \mathbb{Z}\right]$ fortgesetzt werden, die die obige Rekursion für alle $n \in \mathbb{Z}$ erfüllt.

b) Für die erzeugende Funktion $f:=\sum_{n>0} f_{n} X^{n} \in \mathbb{Q}(X) \cap \mathbb{Q}[[X]]$ zeige man: Es gilt $\sum_{n \geq 1} f_{-n} X^{n}=-f\left(\frac{1}{X}\right) \in \mathbb{Q}(X)$. Gilt diese Identität auch in $\mathbb{Q}[[X]]$ ?

\section{(22.5) Aufgabe: Rationale Funktionen.}

Man zeige: Für $f:=\sum_{n \geq 0} f_{n+1} X^{n} \in \mathbb{C}[[X]]$ sind äquivalent:

i) Es gibt $g \in \mathbb{C}(X) \cap \mathbb{C}[[X]]$ mit $f=\frac{\partial}{\partial X} g \cdot \frac{1}{g} \in \mathbb{C}(X)$.

ii) Es gilt $\exp \left(\sum_{n \geq 1} \frac{f_{n}}{n} X^{n}\right) \in \mathbb{C}(X) \cap \mathbb{C}[[X]]$.

iii) Es gibt nicht notwendig verschiedene $a_{1}, \ldots, a_{k}, b_{1}, \ldots, b_{l} \in \mathbb{C}$, für geeignete $k, l \in \mathbb{N}_{0}$, so daß $a_{n}=\sum_{i=1}^{k} a_{i}^{n}-\sum_{j=1}^{l} b_{j}^{n}$, für alle $\in \mathbb{N}$, gilt.

\section{(22.6) Aufgabe: Quasipolynome.}

Eine Abbildung $h: \mathbb{N}_{0} \rightarrow \mathbb{C}$ heißt ein Quasipolynom vom Grad $d \in \mathbb{N}$ und Quasiperiode $k \in \mathbb{N}$, falls es für alle $a \in\{0, \ldots, k-1\}$ ein Polynom $h_{a} \in$ $\mathbb{Q}[X]_{\leq d}$ gibt, so daß $h(n)=h_{a}(n)$ für alle $n \in \mathbb{N}_{0}$ mit $n \equiv a(\bmod k)$ gilt; dabei habe mindestens eines der Polynome $h_{a}$ den Grad $d$. Man zeige die Äquivalenz der folgenden Aussagen:

i) Die Abbildung $h$ ist ein Quasipolynom vom Grad $d-1$ und Quasiperiode $k$.

ii) Es ist $\sum_{n \geq 0} h(n) X^{n}=\frac{p}{q} \in \mathbb{C}(X) \cap \mathbb{C}[[X]]$, wobei $q \in \mathbb{C}[X]$ den Grad $d$ und nur Nullstellen $a \in \mathbb{C}$ mit $a^{k}=1$ hat, und $p \in \mathbb{C}[X]_{\leq d-1}$ teilerfremd zu $q$ ist. 
(22.7) Aufgabe: Rekursion.

Man gebe geschlossene Formeln für die wie folgt rekursiv definierten $a_{n} \in \mathbb{Z}$ an:

a) Es seien $a_{0}:=2$ und $a_{1}:=3$ sowie $a_{n+2}:=3 a_{n+1}-2 a_{n}$ für $n \in \mathbb{N}_{0}$.

b) Es seien $a_{0}:=0$ und $a_{1}:=2$ sowie $a_{n+2}:=4 a_{n+1}-4 a_{n}$ für $n \in \mathbb{N}_{0}$.

c) Es seien $a_{0}:=5$ und $a_{1}:=12$ sowie $a_{n+2}:=4 a_{n+1}-3 a_{n}-2^{n}$ für $n \in \mathbb{N}_{0}$.

(22.8) Aufgabe: Lineare Rekursion.

Für $n \in \mathbb{N}_{0}$ sei $r_{n}:=(1+\sqrt{3})^{n}+(1-\sqrt{3})^{n} \in \mathbb{R}$. Man zeige: Es ist $r_{n} \in \mathbb{N}$ und es gilt $2^{\left\lfloor\frac{n}{2}\right\rfloor+1} \mid r_{n}$.

(22.9) Aufgabe: Quadratwurzeln.

Es sei $1 \neq d \in \mathbb{N}$ quadratfrei.

a) Man zeige: Es gibt eindeutig bestimmte Folgen $\left[a_{n} \in \mathbb{Z} ; n \in \mathbb{N}_{0}\right]$ und $\left[b_{n} \in\right.$ $\left.\mathbb{Z} ; n \in \mathbb{N}_{0}\right]$, so daß $(1+\sqrt{d})^{n}=a_{n}+b_{n} \cdot \sqrt{d} \in \mathbb{R}$ für alle $n \in \mathbb{N}_{0}$ gilt.

b) Man zeige: Die zugehörigen erzeugenden Funktionen $\sum_{n>0} a_{n} X^{n} \in \mathbb{Q}[[X]]$ und $\sum_{n>0} b_{n} X^{n} \in \mathbb{Q}[[X]]$ sind rationale Funktionen, und schreibe sie explizit als Quotient von Polynomen.

(22.10) Aufgabe: Drohnen und Königinnen.

Die Männchen der Honigbiene werden als Drohnen bezeichnet. Eine Drohne enwickelt sich aus einem unbefruchteten Ei, das ausschließlich Erbgut einer Bienenkönigin enhält, während eine Königin sich aus einem befruchteten Ei entwickelt, das das Erbgut einer Drohne und einer anderen Königin enthält. Wieviele Vorfahren $n$-ter Stufe (also Eltern, Großeltern, ...) hat eine Drohne?

\section{(22.11) Aufgabe: Fibonacci-Zahlen.}

Für $n \in \mathbb{N}_{0}$ sei $F_{n} \in \mathbb{N}_{0}$ die $n$-te Fibonacci-Zahl. Man zeige:

a) Für $n \in \mathbb{N}_{0}$ gelten $\sum_{k=0}^{n} F_{k}=F_{n+2}-1$ und $\sum_{k=1}^{n} F_{2 k-1}=F_{2 n}$, sowie $\sum_{k=0}^{n} F_{k}^{2}=F_{n} F_{n+1}$ und $\sum_{k=0}^{n} F_{k} F_{n-k}=\frac{1}{5}\left(2 n F_{n+1}-(n+1) F_{n}\right)$.

b) Für $n \in \mathbb{N}$ gilt $F_{n+1} F_{n-1}-F_{n}^{2}=(-1)^{n}$.

c) Für $k \in \mathbb{N}$ und $n \in \mathbb{N}_{0}$ gelten $F_{n+k}=F_{k} F_{n+1}+F_{k-1} F_{n}$ und $F_{n} \mid F_{k n}$.

d) Man zeige: Jede natürliche Zahl $1 \neq m \in \mathbb{N}$ hat eine eindeutige Darstellung der Form $m=\sum_{n \geq 1} a_{n} F_{n}$, wobei $a_{n} \in\{0,1\}$ und $a_{n} a_{n+1}=0$ für alle $n \in \mathbb{N}$.

(22.12) Aufgabe: Lucas-Zahlen.

Die Folge $\left[L_{n} \in \mathbb{N}_{0} ; n \in \mathbb{N}\right]$ der Lucas-Zahlen ist definiert durch $L_{n+2}:=$ $L_{n}+L_{n+1}$, für alle $n \in \mathbb{N}_{0}$, wobei $L_{0}:=2$ und $L_{1}:=1$.

a) Man gebe eine geschlossene Formel für $L_{n}$ an.

b) Man zeige: Es gilt $L_{n}=F_{n-1}+F_{n+1}$, für alle $n \in \mathbb{N}$, und $F_{2 n}=F_{n} L_{n}$, für alle $n \in \mathbb{N}_{0}$; dabei ist $F_{n} \in \mathbb{N}_{0}$ die $n$-te Fibonacci-Zahl. 
c) An einem runden Tisch sitzen $n \geq 2$ Personen. Wieviele Teilmengen dieser Personen gibt es, unter denen sich keine zwei benachbarte Personen befinden?

\section{(22.13) Aufgabe: Erzeugende Funktion der Fibonacci-Zahlen.}

a) Für $n \in \mathbb{N}_{0}$ sei $F_{n} \in \mathbb{N}_{0}$ die $n$-te Fibonacci-Zahl. Man zeige: Die erzeugende Funktion $F^{\prime}:=\sum_{n>0} F_{2 n} X^{n} \in \mathbb{Q}[[X]]$ ist eine rationale Funktion, und schreibe sie explizit als Quotient von Polynomen.

b) Es sei $\left[g_{n} \in \mathbb{N}_{0} ; n \in \mathbb{N}_{0}\right]$ die durch $g_{0}:=1$ und $g_{n}:=\sum_{i=0}^{n-1}(n-i) g_{i}$, für $n \geq 1$, rekursiv definierte Folge. Man zeige: Die erzeugende Funktion $\sum_{n \geq 0} g_{n} X^{n} \in \mathbb{Q}[[X]]$ ist eine rationale Funktion, schreibe sie explizit als Quotient von Polynomen, und gebe eine geschlossene Formel für $g_{n}$ an.

\section{(22.14) Aufgabe: Schachbrett und Domino-Steine.}

Es sei $a_{n} \in \mathbb{N}_{0}$ die Anzahl der Überdeckungen eines $(3 \times n)$-Schachbrettes mit $(2 \times 1)$-Domino-Steinen, für $n \in \mathbb{N}_{0}$. Man bestimme die zugehörige erzeugende Funktion, und gebe eine geschlossene Formel für $a_{n}$ an.

Hinweis. Man betrachte auch die Anzahl der Überdeckungen, bei denen ein Eckfeld frei bleibt.

\section{(22.15) Aufgabe: Ballot-Problem.}

Vor einer Theaterkasse warten $2 n$ Personen, für $n \in \mathbb{N}_{0}$, um jeweils eine Karte für 10 Euro zu kaufen. Von ihnen haben $n$ Personen einen 10-Euro-Schein, die anderen nur einen 20-Euro-Schein Wechselgeld zur Verfügung. Wieviele Warteschlangen gibt es, so daß der Kassierer stets passend herausgeben kann, wenn er zu Beginn kein Wechselgeld zur Verfügung hat?

\section{(22.16) Aufgabe: Dyck-Wege.}

Für $n \in \mathbb{N}_{0}$ betrachte man ein quadratisches Gitter mit der Kantenlänge $n$. Ein kürzester Weg im Gitter von der linken unteren zur rechten oberen Ecke, der nie die Diagonale übertritt, heißt ein Dyck-Weg. Man bestimme die Anzahl der Dyck-Wege in Abhängigkeit von $n$.

\section{(22.17) Aufgabe: Ebene binäre Bäume.}

Ein ebener binärer Baum ist eine endliche Menge $V$ von Ecken, zusammen mit einer Wurzel $v \in V$ und einer geordneten Partition $V \backslash\{v\}=V_{1} \dot{\cup} V_{2}$, wobei die Unterbäume $V_{1}$ und $V_{2}$ wiederum ebene binäre Bäume sind. Ein ebener binärer Baum heißt strikt binär, falls entweder $V_{1}=\emptyset=V_{2}$ oder $V_{1} \neq$ $\emptyset \neq V_{2}$ gilt, und dies auch für alle Unterbäume gilt. Ebene binäre Bäume mit Eckenmengen $V$ bzw. $W$ heißen isomorph, falls es eine Bijektion $\pi: V \rightarrow W$ gibt, die Isomorphismen $\pi: V_{i} \rightarrow W_{i}$, für $i \in\{1,2\}$, induziert.

a) Man bestimme die Anzahl der Isomorphieklassen ebener binärer Bäume mit $n \in \mathbb{N}_{0}$ Ecken. 
b) Man bestimme ebenso die Anzahl der Isomorphieklassen ebener strikt binärer Bäume mit $2 n+1$ Ecken.

(22.18) Aufgabe: Triangulierung.

Auf wieviele Weisen kann man ein konvexes $n$-Eck, für $n \geq 3$, durch Einfügen von sich im Inneren nicht schneidenden Diagonalen in $n-2$ Dreiecke zerlegen?

\section{(22.19) Aufgabe: Schröder-Problem.}

Für $n \in \mathbb{N}$ ist eine vollständige binäre Partition einer $n$-elementigen Menge $N$ wie folgt rekursiv definiert: Für $n \geq 2$ ist eine vollständige binäre Partition von $N$ eine eine disjunkte Zerlegung $N=N^{\prime} \cup N^{\prime \prime}$ mit $N^{\prime} \neq \emptyset \neq N^{\prime \prime}$, zusammen mit jeweils einer vollständigen binären Partition von $N^{\prime}$ und $N^{\prime \prime}$; für $n=1$ ist $N$ die einzige vollständige binäre Partition von $N$.

Es seien $s(n) \in \mathbb{N}_{0}$ die Anzahl der vollständigen binären Partitionen einer $n$ elementigen Menge, und $s:=\sum_{n \geq 1} \frac{s(n)}{n !} X^{n} \in \mathbb{Q}[[X]]$ die zugehörige exponentiell erzeugende Funktion. Man zeige: Es gilt $s(x)=1-\sqrt{1-2 x}$, für alle $x \in \mathbb{C}$ in einer hinreichend kleinen offenen Umgebung von 0. Daraus bestimme man eine geschlossene Formel für $s(n)$.

\section{(22.20) Aufgabe: Algebraische Funktionen.}

a) Man zeige: Es gilt $\sum_{n \geq 0}\left(\begin{array}{c}2 n \\ n\end{array}\right) x^{n}=\frac{1}{\sqrt{1-4 x}}$, für alle $x \in \mathbb{C}$ in einer hinreichend kleinen offenen Umgebung von 0.

b) Man gebe eine analytische Beschreibung von $\sum_{n \geq 0}\left(\begin{array}{c}2 n+1 \\ n\end{array}\right) x^{n}$ an.

c) Man zeige: Für alle $n \in \mathbb{N}_{0}$ gilt $\sum_{k=0}^{n}\left(\begin{array}{c}2 k \\ k\end{array}\right)\left(\begin{array}{c}2(n-k) \\ n-k\end{array}\right)=4^{n}$.

\section{(22.21) Aufgabe: Exponentiell erzeugende Funktionen.}

Es sei $\left[g_{n} \in \mathbb{Z} ; n \in \mathbb{N}_{0}\right]$ die durch $g_{n}+2 n g_{n-1}=\sum_{k=0}^{n}\left(\begin{array}{l}n \\ k\end{array}\right) g_{k} g_{n-k}$, für $n \geq 1$, sowie $g_{0}:=0$ und $g_{1}:=1$, rekursiv definierte Folge. Man gebe eine geschlossene Formel für $g_{n}$ an.

\section{(22.22) Aufgabe: Involutionen.}

Für $n \in \mathbb{N}_{0}$ sei $i_{n}:=\left|\left\{\pi \in \mathcal{S}_{n} ; \pi^{2}=1\right\}\right|$ die Anzahl der Involutionen in $\mathcal{S}_{n}$.

a) Man zeige: Es gilt die Rekursion $i_{n+1}:=i_{n}+n \cdot i_{n-1}$, für alle $n \in \mathbb{N}$.

b) Man zeige: Es gilt $\sum_{n \geq 0} \frac{i_{n}}{n !} X^{n}=\exp \left(X+\frac{X^{2}}{2}\right) \in \mathbb{Q}[[X]]$.

c) Man zeige: Für $n \in \mathbb{N}_{0}$ gilt $i_{n}=\sum_{k \geq 0}\left(\begin{array}{c}n \\ 2 k\end{array}\right) \cdot \frac{(2 k) !}{2^{k} \cdot k !} \in \mathbb{Q}$.

d) Man gebe eine geschlossene Formel für $\sum_{k=0}^{n}(-1)^{k}\left(\begin{array}{l}n \\ k\end{array}\right) i_{k} \in \mathbb{Z}$ an.

Hinweis zu c). Man benutze $\exp \left(X+\frac{X^{2}}{2}\right)=\exp (X) \exp \left(\frac{X^{2}}{2}\right)$. 
(22.23) Aufgabe: Arrangements.

Es seien $n, k \in \mathbb{N}_{0}$, und $a_{n, k} \in \mathbb{N}_{0}$ die Anzahl der $k$-Arrangements einer $n$ elementigen Menge. Weiter sei $b_{n}:=\sum_{k>0} a_{n, k} \in \mathbb{N}_{0}$ die Anzahl aller Arrangements einer $n$-elementigen Menge. Für die zugehörigen exponentiell erzeugenden Funktionen zeige man:

a) Für $n \in \mathbb{N}_{0}$ gilt $\sum_{k \geq 0} \frac{a_{n, k}}{k !} X^{k}=(1+X)^{n} \in \mathbb{Q}[[X]]$.

b) Für $k \in \mathbb{N}_{0}$ gilt $\sum_{n \geq 0} \frac{a_{n, k}}{n !} X^{n}=X^{k} \cdot \exp \in \mathbb{Q}[[X]]$.

c) Es gilt $\sum_{n \geq 0} \frac{b_{n}}{n !} X^{n}=\frac{\exp }{1-X} \in \mathbb{Q}[[X]]$.

\section{(22.24) Aufgabe: Mengen von Abbildungen.}

Für $n \in \mathbb{N}_{0}$ sei $a_{n} \in \mathbb{N}_{0}$ die Anzahl aller Abbildungen $f: N \rightarrow N$, wobei $N:=\{1, \ldots, n\}$, mit $f(N)=\{1, \ldots, i\}$ für ein $i \in N$.

a) Man zeige: Für die exponentiell erzeugende Funktion $\widetilde{A}:=\sum_{n \geq 0} \frac{a_{n}}{n !} X^{n} \in$ $\mathbb{Q}[[X]]$ gilt $\widetilde{A}=\frac{1}{2-\exp } \in \mathbb{Q}[[X]]$.

b) Man zeige: Es gilt $a_{n}=\sum_{k \geq 0} \frac{k^{n}}{2^{k+1}}$, für alle $n \in \mathbb{N}_{0}$.

\section{(22.25) Aufgabe: Bernoulli-Zahlen.}

Es sei $\left[B_{n} \in \mathbb{Q} ; n \in \mathbb{N}_{0}\right]$ die durch $B_{0}:=1$ und $\sum_{k=0}^{n}\left(\begin{array}{c}n+1 \\ k\end{array}\right) B_{k}=0$, für $n \in \mathbb{N}$, definierte Folge der Bernoulli-Zahlen.

a) Man zeige: Es gilt $\widetilde{B}(X):=\sum_{n \geq 0} \frac{B_{n}}{n !} X^{n}=\frac{X}{\exp (X)-1} \in \mathbb{Q}[[X]]$. Daraus folgere man: Es ist $\widetilde{B}(X) \in \mathbb{C}[[X]]^{\infty}$, und es gilt $B_{2 n+1}=0$ für alle $n \in \mathbb{N}_{0}$.

b) Es sei $b_{n}(T):=\sum_{k=0}^{n}\left(\begin{array}{l}n \\ k\end{array}\right) B_{k} T^{n-k} \in \mathbb{Q}[T]$ das $n$-te Bernoulli-Polynom, für $n \in \mathbb{N}_{0}$. Man zeige: Für $n \in \mathbb{N}_{0}$ gilt $\frac{\partial}{\partial T}\left(b_{n+1}(T)\right)=(n+1) \cdot b_{n}(T) \in \mathbb{Q}[T]$.

c) Für die exponentiell erzeugende Funktion $\widetilde{B}(T, X):=\sum_{n \geq 0} \frac{b_{n}(T)}{n !} X^{n} \in$ $\mathbb{Q}[T][[X]]$ zeige man: Es gilt $\widetilde{B}(T, X):=\widetilde{B}(X) \cdot \exp (T X)=\frac{X \cdot \exp (T X)}{\exp (X)-1}$.

(22.26) Aufgabe: Erzeugende Funktion der Potenzsummen.

Für $k \geq 1$ sei $s_{k} \in \mathbb{Q}[T]$, so daß $s_{k}(n)=\sum_{i=0}^{n-1} i^{k}$ für alle $n \in \mathbb{N}_{0}$.

a) Für die exponentiell erzeugende Funktion $\widetilde{S}(T, X):=\sum_{k \geq 0} \frac{s_{k}(T)}{k !} X^{k} \in$ $\mathbb{Q}[T][[X]]$ zeige man: Es gilt $\widetilde{S}(T, X)=\frac{\exp (T X)-1}{\exp (X)-1} \in \mathbb{Q}[T][[X]]$, und folglich $X \cdot \widetilde{S}(T, X)=\widetilde{B}(T, X)-\widetilde{B}(0, X) \in \mathbb{Q}[T][[X]] ;$ dabei ist $\widetilde{B}(T, X) \in \mathbb{Q}[T][[X]]$ die exponentiell erzeugende Funktion der Bernoulli-Polynome.

b) Daraus folgere man: Es gilt $s_{k}(T)=\frac{1}{k+1} \sum_{i=0}^{k}\left(\begin{array}{c}k+1 \\ i\end{array}\right) B_{i} T^{k+1-i} \in \mathbb{Q}[T]$.

\section{(22.27) Aufgabe: Konjugierte Partitionen.}

Für eine Partition $\lambda=\left[\lambda_{1}, \lambda_{2}, \ldots\right] \vdash n \in \mathbb{N}_{0}$ und die zugehörige konjugierte Partition $\lambda^{\prime}=\left[\lambda_{1}^{\prime}, \lambda_{2}^{\prime}, \ldots\right] \vdash n$ zeige man: 
a) Es gilt $\sum_{i \geq 1}(i-1) \lambda_{i}=\sum_{i \geq 1}\left(\begin{array}{c}\lambda_{i}^{\prime 2} \\ 2\end{array}\right)$.

b) Es gelten $\sum_{i \geq 1}\left\lceil\frac{\lambda_{2 i-1}}{2}\right\rceil=\sum_{i \geq 1}\left\lceil\frac{\lambda_{2 i-1}}{2}\right\rceil$ und $\sum_{i \geq 1}\left\lfloor\frac{\lambda_{2 i-1}}{2}\right\rfloor=\sum_{i \geq 1}\left\lceil\frac{\lambda_{2 i}}{2}\right\rceil$ sowie $\sum_{i \geq 1}\left\lfloor\frac{\lambda_{2 i}}{2}\right\rfloor=\sum_{i \geq 1}\left\lfloor\frac{\lambda_{2 i}}{2}\right\rfloor$.

\section{(22.28) Aufgabe: Durfee-Quadrat.}

a) Man zeige: Es gilt die Identität

$$
\prod_{i \geq 1} \frac{1}{1-Y X^{i}}=\sum_{k \geq 0}\left(\prod_{i=1}^{k} \frac{1}{\left(1-X^{i}\right)\left(1-Y X^{i}\right)}\right) X^{k^{2}} Y^{k} \in \mathbb{Q}[[X]][[Y]] .
$$

b) Man gebe einen kombinatorischen Beweis für die folgende Identität an:

$$
\prod_{i \geq 1}\left(1+Y X^{2 i-1}\right)=\sum_{k \geq 0}\left(\prod_{i=1}^{k} \frac{1}{1-X^{2 i}}\right) X^{k^{2}} Y^{k} \in \mathbb{Q}[[X]][[Y]]
$$

(22.29) Aufgabe: Selbst-konjugierte Partitionen.

Für $n \in \mathbb{N}_{0}$ sei $e_{n} \in \mathbb{N}_{0}$ die Anzahl der selbst-konjugierten Partitionen von $n$ in lauter gerade Teile. Man gebe eine geschlossene Formel für die erzeugende Funktion $\sum_{n \geq 0} e_{n} X^{n} \in \mathbb{Q}[[X]]$ an.

(22.30) Aufgabe: Erzeugende Funktionen für Partitionsanzahlen.

Für $n \in \mathbb{N}_{0}$ seien $f_{n} \in \mathbb{N}_{0}$ die Anzahl der Partitionen von $n$, in denen der Teil $i$ höchstens $i$-mal vorkommt, für alle $i \in\{1, \ldots, n\}$, und $g_{n} \in \mathbb{N}_{0}$ die Anzahl der Partitionen von $n$, die keinen Teil der Form $i(i+1)$ haben, für ein $i \in \mathbb{N}$. Man zeige: Es gilt $f_{n}=g_{n}$, für alle $\in \mathbb{N}_{0}$, und gebe ein geschlossene Formel für die erzeugende Funktion $\sum_{n \geq 0} f_{n} X^{n}=\sum_{n \geq 0} g_{n} X^{n} \in \mathbb{Q}[[X]]$ an.

\section{Exercises for Part V (in German)}

(23.1) Aufgabe: Double Counting.

Für $n \in \mathbb{N}$ seien $\tau(n):=|\{i \in \mathbb{N} ; i \mid n\}| \in \mathbb{N}$ die Anzahl der Teiler von $n$, und $\bar{\tau}(n):=\frac{1}{n} \cdot \sum_{i=1}^{n} \tau(i) \in \mathbb{Q}$. Man zeige: Es gilt $\lim _{n \rightarrow \infty} \frac{\bar{\tau}(n)}{\ln (n)}=1$.

Hinweis. Man zeige $\lim _{n \rightarrow \infty} \frac{\bar{\tau}(n)}{h_{n}}=1$, wobei $h_{n}:=\sum_{i=1}^{n} \frac{1}{i} \in \mathbb{Q}$ die $n$-te harmonische Zahl bezeichne, und benutze $\lim _{n \rightarrow \infty} \frac{h_{n}}{\ln (n)}=1$.

\section{(23.2) Aufgabe: Parkers Lemma.}

Es sei $G \leq \mathcal{S}_{n}$, wobei $n \in \mathbb{N}$. Für $k \in \mathbb{N}$ sei $\mathcal{C}_{k} \subseteq \mathcal{S}_{n}$ die Menge der $k$-Zykel, die in einem beliebigen Element von $G$ vorkommen.

a) Man zeige: $\mathcal{S}_{n}$ operiert auf sich durch Konjugation $\mathcal{S}_{n} \rightarrow \mathcal{S}_{n}: \sigma \mapsto \pi \sigma \pi^{-1}$, für alle $\pi \in \mathcal{S}_{n}$. Daraus folgere man: $G$ operiert auf $\mathcal{C}_{k}$ durch Konjugation. 
b) Man zeige: Die $G$-Mengen $\mathcal{C}_{1}$ und $\{1, \ldots, n\}$ sind äquivalent. Was hat also die Aussage in (c) mit dem Cauchy-Frobenius-Burnside-Lemma zu tun?

c) Für die Anzahl der $G$-Bahnen auf $\mathcal{C}_{k}$ zeige man $\left|G \backslash \mathcal{C}_{k}\right|=\frac{1}{|G|} \cdot \sum_{\pi \in G} k a_{k}(\pi)$; dabei sei $a_{k}(\pi) \in \mathbb{N}_{0}$ die Anzahl der Teile $k$ im Zykeltyp von $\pi$.

Hinweis zu c). Man bestimme den Stabilisator eines Zykels in $\mathcal{C}_{k}$, und betrachte eine geeignete $(|G| \times n)$-Matrix wie im Beweis des Cauchy-FrobeniusBurnside-Lemmas.

\section{(23.3) Aufgabe: Türme auf dem Schachbrett.}

Für $n \in\{1, \ldots, 5\}$ betrachte man ein $(n \times n)$-Schachbrett, dessen Vorder- und Rückseite nicht unterschieden werden können. Wieviele Muster gibt es, $n$ Türme so zu verteilen, daß sie sich nicht gegenseitig schlagen können? Können Sie eine allgemeine Formel für diese Anzahl angeben?

\section{(23.4) Aufgabe: Konstruktionen mit Permutationsgruppen.}

Es seien $G \leq \mathcal{S}_{n}$ und $H \leq \mathcal{S}_{m}$ Permutationsgruppen, für $n, m \in \mathbb{N}$, mit zugehörigen Zykelindizes $c_{G} \in \mathbb{Q}\left[X_{1}, \ldots, X_{n}\right]$ bzw. $c_{H} \in \mathbb{Q}\left[X_{1}, \ldots, X_{m}\right]$. Man bestimme jeweils den Zykelindex für die natürliche Operation

a) des direkten Produkts $G \times H$ auf $n+m$ Punkten, und

b) des Kranzprodukts $G$ 乙 $H$ auf $n m$ Punkten.

\section{(23.5) Aufgabe: Zykelindex symmetrischer Gruppen.}

Unter Benutzung des Zykelindexes für $\mathcal{S}_{n}$, für $n \in \mathbb{N}_{0}$, bestimme man (erneut) die exponentiell erzeugende Funktion $\sum_{n \geq 0} \frac{i_{n}}{n !} X^{n} \in \mathbb{Q}[[X]]$ der Anzahl $i_{n}:=$ $\left|\left\{\pi \in \mathcal{S}_{n} ; \pi^{2}=1\right\}\right| \in \mathbb{N}$ der Involutionen in $\mathcal{S}_{n}$.

(23.6) Aufgabe: Zykelindex alternierender Gruppen.

Für $n \geq 2$ bestimme man den Zykelindex der alternierenden Gruppe $\mathcal{A}_{n}$.

Hinweis. Eine Permutation $\pi \in \mathcal{S}_{n}$ ist genau dann ungerade, wenn für ihren Zykeltyp $\left[n^{a_{n}(\pi)}, \ldots, 1^{a_{1}(\pi)}\right]$ gilt: $1+(-1)^{\sum_{i \geq 1} a_{2 i}(\pi)}=0$.

\section{(23.7) Aufgabe: Zykelindex von Diedergruppen.}

a) Für den Zykelindex der zyklischen Gruppe $C_{n}:=\langle(1, \ldots, n)\rangle \leq \mathcal{S}_{n}$, wobei $n \in \mathbb{N}$, zeige man: Es gilt $c_{C_{n}}=\frac{1}{n} \cdot \sum_{d \mid n} \varphi(d) X_{d}^{\frac{n}{d}} \in \mathbb{Q}\left[X_{1}, \ldots, X_{n}\right]$; dabei sei $\varphi(n):=|\{k \in\{0, \ldots, n-1\} ; \operatorname{ggT}(k, n)=1\}| \in \mathbb{N}$ die Euler-Funktion.

b) Für den Zykelindex $c_{D_{2 n}} \in \mathbb{Q}\left[X_{1}, \ldots, X_{n}\right]$ der Diedergruppe $D_{2 n} \leq \mathcal{S}_{n}$, wobei $n \geq 3$, zeige man: Es gilt

$$
c_{D_{2 n}}=\frac{1}{2} c_{C_{n}}+ \begin{cases}\frac{1}{2} X_{1} X_{2}^{\frac{n-1}{2}}, & \text { falls } n \text { ungerade } \\ \frac{1}{4}\left(X_{2}^{\frac{n}{2}}+X_{1}^{2} X_{2}^{\frac{n}{2}-1}\right), & \text { falls } n \text { gerade }\end{cases}
$$




\section{(23.8) Aufgabe: Kleiner Satz von Fermat II.}

Es seien $n \in \mathbb{N}$ und $p \in \mathbb{N}$ eine Primzahl. Man zeige: Es gilt $n^{p} \equiv n(\bmod p)$.

Hinweis. Man benutze den Zykelindex der zyklischen Gruppe $C_{p}$.

\section{(23.9) Aufgabe: Würfel.}

Man bestimme die Drehgruppe eines Würfels im Euklidischen Raum $\mathbb{R}^{3 \times 1}$ jeweils als Gruppe von Permutationen seiner i) acht Ecken, ii) zwölf Kanten, iii) sechs Flächen, iv) vier Raumdiagonalen, und gebe die jeweiligen Zykelindizes an. $\mathrm{Zu}$ welcher bekannten Gruppe ist die Drehgruppe isomorph?

\section{(23.10) Aufgabe: Tetraeder.}

a) Man bestimme die Symmetriegruppe eines gleichseitigen Tetraeders im Euklidischen Raum $\mathbb{R}^{3 \times 1}$ jeweils als Gruppe von Permutationen seiner i) vier Ecken, ii) sechs Kanten, iii) vier Flächen, und gebe die jeweiligen Zykelindizes an. Wieviele Drehungen gibt es? Zu welchen bekannten Gruppen sind die Symmetriegruppe und die Drehgruppe isomorph?

b) Man bestimme die Anzahl der verschiedenen Färbungen der vier Ecken des Tetraeders mit bis zu vier Farben, bezüglich der vollen Symmetriegruppe und der Drehgruppe. Wie kann man das Ergebnis geometrisch interpretieren?

\section{(23.11) Aufgabe: Prisma.}

Man bestimme die Symmetriegruppe eines geraden Prismas über einem gleichseitigen Dreieck im Euklidischen Raum $\mathbb{R}^{3 \times 1}$ jeweils als Gruppe von Permutationen seiner i) sechs Ecken, ii) neun Kanten, iii) fünf Flächen, und gebe die jeweiligen Zykelindizes an. Wieviele Drehungen gibt es? Zu welchen bekannten Gruppen sind die Symmetriegruppe und die Drehgruppe isomorph?

\section{(23.12) Aufgabe: Gewichtsindex.}

Es seien $G$ eine endliche Gruppe, die treu auf $N:=\{1, \ldots, n\}$ operiere, und $K:=$ $\{1, \ldots, k\}$, wobei $n \in \mathbb{N}_{0}$ und $k \in \mathbb{N}_{0}$. Weiter seien $S \subseteq \operatorname{Inj}(N, K)$ eine Repräsentantenmenge für $G \backslash \operatorname{Inj}(N, K)$ und $e_{n}\left(Y_{1}, \ldots, Y_{k}\right):=\sum_{J \subseteq K,|J|=n} \prod_{j \in J} Y_{j} \in$ $R:=\mathbb{Z}\left[Y_{1}, \ldots, Y_{k}\right]$ das elementar-symmetrische Polynom vom Grad $n$.

Für den Gewichtsindex von $G \backslash \operatorname{Inj}(N, K)$ zeige man: Es gilt $\sum_{f \in S} \prod_{i=1}^{n} Y_{f(i)}=$ $\frac{n !}{|G|} \cdot e_{n}\left(Y_{1}, \ldots, Y_{k}\right) \in R$. Welche bekannten Formeln erhält man daraus für die Spezialisierung $R \rightarrow \mathbb{Z}: Y_{j} \mapsto 1$, für alle $j \in K$, und $G=\{1\}$ bzw. $G=\mathcal{S}_{N}$ ?

\section{(23.13) Aufgabe: Graphen.}

Ein (endlicher einfacher) Graph $\Gamma$ besteht aus einer endlichen Menge $\mathcal{V}$ von Ecken und einer Menge von 2-elementigen Teilmengen von $\mathcal{V}$, die Kanten genannt werden. Ein Graph heißt zusammenhängend, falls man von jeder Ecke auf einem Weg, also einer Kantenfolge, zu jeder anderen Ecke gelangen kann. Graphen $\Gamma$ und $\Gamma^{\prime}$ heißen isomorph, falls es eine Bijektion zwischen den Ecken von $\Gamma$ und $\Gamma^{\prime}$ gibt, die eine Bijektion der Kanten von $\Gamma$ und $\Gamma^{\prime}$ bewirkt. 
a) Es seien $r_{n} \in \mathbb{N}$ und $s_{n} \in \mathbb{N}$ die Anzahl der Isomorphieklassen von Graphen bzw. von zusammenhängenden Graphen mit $n \in \mathbb{N}$ Ecken. Man zeige: Für die erzeugenden Funktionen $r:=\sum_{n \geq 1} r_{n} Y^{n} \in \mathbb{Q}[[Y]]$ und $s:=\sum_{n \geq 1} s_{n} Y^{n} \in$ $\mathbb{Q}[[Y]]$ gilt die Funktionalgleichung $r(Y)=\exp \left(\sum_{k \geq 1} \frac{s\left(Y^{k}\right)}{k}\right) \in \mathbb{Q}[[Y]]$.

b) Es sei $c_{\mathcal{S}_{n}}^{(2)} \in \mathbb{Q}\left[X_{1}, \ldots, X_{\left(\begin{array}{c}n \\ 2\end{array}\right)}\right]$ der Zykelindex der Operation von $\mathcal{S}_{n}$ auf den 2-elementigen Teilmengen von $\{1, \ldots, n\}$. Man zeige: Die Anzahl $r_{n, k} \in \mathbb{N}_{0}$ der Isomorphieklassen von Graphen mit $n \in \mathbb{N}$ Ecken und $k \in\left\{0, \ldots,\left(\begin{array}{c}n \\ 2\end{array}\right)\right\}$ Kanten ist der Koeffizient von $Y^{k}$ im Polynom $c_{\mathcal{S}_{n}}^{(2)}\left(1+Y, \ldots, 1+Y^{\left(\begin{array}{l}n \\ 2\end{array}\right)}\right) \in \mathbb{Q}[Y]$. Daraus folgere man: Es gilt $r_{n}=c_{\mathcal{S}_{n}}^{(2)}(2, \ldots, 2)$.

c) Man bestimme die Anzahl der Isomorphieklassen von Graphen bzw. von zusammenhängenden Graphen mit $n \in\{1, \ldots, 5\}$ Ecken und $k \in\left\{0, \ldots,\left(\begin{array}{l}n \\ 2\end{array}\right)\right\}$ Kanten, und stelle die zugehörigen Graphen graphisch dar.

\section{(23.14) Aufgabe: Bäume mit Wurzel.}

Ein Baum ist ein zusammenhängender kreisfreier Graph; eine Wurzel ist eine ausgezeichnete Ecke. Zwei Bäume mit Wurzel heißen isomorph, falls es einen Graphenisomorphismus zwischen ihnen gibt, der die Wurzeln respektiert.

Es sei $t_{n} \in \mathbb{N}$ die Anzahl der Isomorphieklassen von Bäumen mit Wurzel auf $n \in \mathbb{N}$ Ecken. Man zeige: Die erzeugende Funktion $t:=\sum_{n \geq 1} t_{n} Y^{n} \in \mathbb{Q}[[Y]]$ erfüllt die Funktionalgleichung $t(Y)=Y \cdot \exp \left(\sum_{k>1} \frac{t\left(Y^{k}\right)}{k}\right) \in \mathbb{Q}[[Y]]$. Daraus bestimme man $t_{1}, \ldots, t_{5}$, und stelle die zugehörigen Bäume graphisch dar.

\section{(23.15) Aufgabe: Selbst-duale Muster.}

a) Es seien $G$ eine endliche Gruppe, die treu auf der endlichen Menge $N$ operiere, und $c_{G}$ der zugehörige Zykelindex. Eine Abbildung $f \in \operatorname{Abb}(N,\{1,2\})$ heißt selbst-dual, falls das zugehörige Muster sich bei der Umbenennung durch $\sigma:=$ $(1,2) \in \mathcal{S}_{2}$ nicht ändert, das heißt, wenn $\sigma(f) \in{ }^{G} f$ gilt. Man zeige:

Die Menge $\operatorname{Abb}(N,\{1,2\})^{+}$der selbst-dualen Abbildungen ist eine Vereinigung von $G$-Bahnen, und es gilt $\left|G \backslash \operatorname{Abb}(N,\{1,2\})^{+}\right|=c_{G}(0,2,0,2, \ldots) \in \mathbb{N}_{0}$.

b) Man bestimme die Anzahl selbst-dualer Halsketten mit $n$ Perlen, die zwei verschiedene Farben haben können, für i) $n \geq 3$ ungerade, ii) $n=2^{k}$ mit $k \geq 2$. 


\section{References}

\section{Textbooks}

[1] M. Aigner: Diskrete Mathematik, 6. Aufl., Vieweg Studium Aufbaukurs Mathematik, 2006.

[2] M. Aigner: Discrete mathematics, translated from the 2004 German original by David Kramer, American Mathematical Society, 2007.

[3] M. Auslander, I. Reiten, S. Smalø: Representation theory of Artin algebras, corrected reprint of the 1995 original, Cambridge Studies in Advanced Mathematics 36, Cambridge University Press, 1997.

[4] G. Birkhoff: Lattice theory, 3rd ed., AMS Colloquium Publications 25, 1984.

[5] P. Cameron: Permutation groups, Cambridge University Press, 1999.

[6] M. Hall: Combinatorial theory, 2nd ed., Wiley, 1998.

[7] K. Jacobs, D. Jungnickel: Einführung in die Kombinatorik, 2. Aufl., de Gruyter, 2004.

[8] A. Kerber: Algebraic combinatorics via finite group actions, Bibliographisches Institut Mannheim, 1991.

[9] D. Marcus: Number fields, Springer, 1977.

[10] G. Polya, R. TARJan, D. Woods: Notes on introductory combinatorics, Modern Birkhäuser Classics, 2010.

[11] R. Stanley: Enumerative combinatorics, vol. I, Cambridge Studies in Advanced Mathematics 49, Cambridge University Press, 1997.

[12] R. Stanley: Enumerative combinatorics, vol. II, Cambridge Studies in Advanced Mathematics 62, Cambridge University Press, 1999.

[13] A. Tucker: Applied combinatorics, 3rd ed., Wiley, 1995.

\section{Research papers}

[14] D. Benson, J. Conway: Diagrams for modular lattices, J. Pure Appl. Algebra 37, 1985, 111-116.

[15] B. Coleman, K. Hartshorn: Game, set, math, Math. Mag. 85, 2012, 83-96.

[16] M. Iovanov, G. Koffi: On incidence algebras and their representations, Preprint, 2017, arXiv:1702.03356 [math.RT].

[17] J. KlÜners, G. Malle: A database for number fields, http://galoisdb. math.upb.de, 2011.

[18] K. Lux, J. Müller, M. Ringe: Peakword condensation and submodule lattices: an application of the MeatAxe, J. Symbolic Comput. 17, 1994, $529-544$. 
[19] J. MülLeR: On a theorem by Benson and Conway, J. Pure Appl. Algebra 208, 2007, 89-100.

[20] G. Polya: Kombinatorische Anzahlbestimmungen für Gruppen, Graphen und chemische Verbindungen, Acta Math. 68, 1937, 145-254.

[21] G. Rota: On the foundations of combinatorial theory, I. Theory of Möbius functions, Z. Wahrscheinlichkeit 2, 1964, 340-368.

[22] J. Serre: On a theorem of Jordan, Bull. Amer. Math. Soc. 40, 2003, 429-440. 